\title{
Precision Search for Muon Antineutrino Disappearance Oscillations Using a Dual Baseline Technique
}

\author{
Gary Chia Li Cheng
}

Submitted in partial fulfillment of the

requirements for the degree

of Doctor of Philosophy

in the Graduate School of Arts and Sciences

\section{COLUMBIA UNIVERSITY}


(C) 2013

Gary Chia Li Cheng

All Rights Reserved 


\section{ABSTRACT \\ Precision Search for Muon Antineutrino Disappearance Oscillations Using a Dual Baseline Technique}

\section{Gary Chia Li Cheng}

A search for short baseline muon antineutrino disappearance with the SciBooNE and MiniBooNE experiments at Fermi National Accelerator Laboratory in Batavia, Illinois is presented. Short baseline $\bar{\nu}_{\mu}$ disappearance measurements help constrain sterile neutrino models. The two detectors observe muon antineutrinos from the same beam, therefore the combined analysis of their data sets serves to partially constrain some of the flux and cross section uncertainties. A likelihood ratio method was used to set a $90 \%$ confidence level upper limit on $\bar{\nu}_{\mu}$ disappearance that dramatically improves upon prior sterile neutrino oscillation limits in the $\Delta m^{2}=0.1-100 \mathrm{eV}^{2}$ region. 


\section{Table of Contents}

$\begin{array}{lll}1 \text { Introduction } & 1\end{array}$

2 Neutrinos, Neutrino Oscillations, and Sterile Neutrinos 3

2.1 Introduction to Neutrinos $\ldots \ldots \ldots \ldots \ldots \ldots$

2.2 Neutrino Oscillations . . . . . . . . . . . . . . . . . . . . 4

$2.2 .1 \quad$ Neutrino Measurements $\ldots \ldots \ldots \ldots$. . . . . . . . . . 7

2.3 LSND Observation and Sterile Neutrinos . . . . . . . . . . . . . . . . . 8

2.4 Antineutrino Disappearance . . . . . . . . . . . . . . . . . 10

3 SciBooNE and MiniBooNE Experiments 14

3.1 The Booster Neutrino Beamline (BNB) . . . . . . . . . . . . . . . 14

3.2 The SciBooNE and MiniBooNE Detectors . . . . . . . . . . . . . . . . . 19

3.3 The SciBooNE Detector $\ldots \ldots \ldots \ldots$

3.3.1 The SciBar Sub-detector. . . . . . . . . . . . . . . . . . . . . 19

3.3 .2 The EC Sub-detector . . . . . . . . . . . . . . . . . 23

3.3 .3 The MRD Sub-detector . . . . . . . . . . . . . . . . . . . 24

3.3 .4 Events in SciBooNE . . . . . . . . . . . . . . . . . . . . . 24

3.4 The MiniBoonE Detector . . . . . . . . . . . . . . . . . . . 25

3.4 .1 Events in MiniBoonE . . . . . . . . . . . . . . . . . . 29

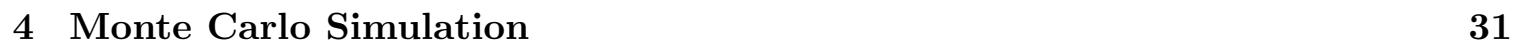

$4.1 \quad$ Monte Carlo Events and Event Weighting . . . . . . . . . . . . . . . . 31

4.2 Booster Neutrino Beamline Antineutrino Flux . . . . . . . . . . . . . . 32 
4.2.1 Proton Beam, Beryllium Target, Beamline Geometry, and ProtonBeryllium Interaction Modeling . . . . . . . . . . . . . . . . . . . . 33

$4.2 .2 \pi$ and $K$ Meson Production Simulation $\ldots \ldots \ldots$

$4.2 .3 \quad$ Simulation of Meson Decays . . . . . . . . . . . . . . . . . . 38

4.3 Neutrino and Antineutrino Interactions f . . . . . . . . . . . . . . . . 40

$4.3 .1 \quad$ Quasielastic Scattering . . . . . . . . . . . . . . . . . . 42

4.3 .2 Meson Production $\ldots \ldots \ldots$. . . . . . . . . . . . 47

$4.3 .3 \quad$ Deep Inelastic Scattering $\ldots \ldots \ldots$. . . . . . . . . . . . . . 48

$4.3 .4 \quad$ Final State Internuclear Interactions . . . . . . . . . . . . . . 48

4.4 Detector Response $\ldots \ldots \ldots \ldots$

$4.4 .1 \quad$ MiniBooNE Detector Response $\ldots \ldots$. . . . . . . . . . . . . . . . 49

$4.4 .2 \quad$ SciBooNE Detector Response $\ldots \ldots \ldots$

5 Event Reconstruction and Selection $\quad 51$

5.1 SciBooNE Event Reconstruction . . . . . . . . . . . . . . . . . . 52

5.1 .1 SciBar Track Reconstruction . . . . . . . . . . . . . . . 52

5.1 .2 EC Cluster Reconstruction $\ldots \ldots \ldots \ldots$

$5.1 .3 \quad$ MRD Track Reconstruction . . . . . . . . . . . . . . . . . . . . . 53

5.1 .4 SB-MRD Matched Track . . . . . . . . . . . . . . 53

5.2 MiniBoonE Event Reconstruction . . . . . . . . . . . . . . . . . 54

5.3 SciBoonE and MiniBooNE Data Sets . . . . . . . . . . . . . . . . 55

$5.3 .1 \quad$ BNB POT Collection $\ldots \ldots \ldots \ldots \ldots$

5.3 .2 Data Quality . . . . . . . . . . . . . . 56

5.4 SciBooNE CC Inclusive Event Selection . . . . . . . . . . . . . . . . 60

5.4 .1 Initial Cuts . . . . . . . . . . . . . . . . . 60

$5.4 .2 \quad$ SciBar Stopped Events . . . . . . . . . . . . . . . . 62

$5.4 .3 \quad$ MRD Stopped Events . . . . . . . . . . . . . . . . . 64

5.4 .4 Data Events . . . . . . . . . . . . . . . . . . . . 66

5.5 MiniBooNE CCQE Event Selection . . . . . . . . . . . . . . . . . . . 68

5.6 Antineutrino Energy Reconstruction . . . . . . . . . . . . . . . 71

5.7 Composition of the Selected MiniBoonE and SciBooNE Events . . . . . . . 73 
5.8 Event Reconstruction and Selection Results . . . . . . . . . . . . . . . . . 81

$\begin{array}{lll}6 & \text { Uncertainties } & 82\end{array}$

6.1 Multsim Method and Reweighting . . . . . . . . . . . . . 83

6.2 Antineutrino Flux Uncertainties . . . . . . . . . . . . . . . . . 86

6.3 Neutrino Interaction Uncertainties $\ldots \ldots \ldots$. . . . . . . . . . . . . . 88

6.4 Detector Uncertainties . . . . . . . . . . . . . . . . . . . . . . . . . . . . . 92

6.4 .1 SciBooNE Detector Uncertainties . . . . . . . . . . . . . . . . 92

6.4 .2 MiniBooNE Detector Uncertainties . . . . . . . . . . . . . . . . . 95

6.5 Statistical Uncertainties . . . . . . . . . . . . . . . . . . . . . . . 98

6.6 Error Matrix $\ldots \ldots \ldots \ldots$

6.6 .1 Fractional Error Matrix . . . . . . . . . . . . . . . . . . . . 98

6.7 Conclusion on Uncertainties $\ldots \ldots \ldots \ldots$

$\begin{array}{lll}7 & \text { Search for Dual Baseline Disappearance } & 110\end{array}$

$7.1 \quad$ Antineutrino Disappearance Oscillation Probability . . . . . . . . . . . . . . 111

$7.2 \quad \chi^{2}$ and $\Delta \chi^{2}$ Test Statistic . . . . . . . . . . . . . . . . . . . . . 112

7.3 Feldman-Cousins Method $\ldots \ldots \ldots \ldots$. . . . . . . . . . . 113

7.4 Frequentist Studies $\ldots \ldots \ldots \ldots$. . . . . . . . . . . . . 115

7.5 Results . . . . . . . . . . . . . . . . . . . . . . . . . 129

$\begin{array}{lll}8 & \text { Conclusions } & 139\end{array}$

\begin{tabular}{lr}
\hline Bibliography & 140
\end{tabular}

A MiniBooNE and SciBooNE Muon Antineutrino Disappearance Publication 


\section{List of Figures}

2.1 The neutrino mass eigenstates in the normal and inverted neutrino mass hierarchies. The flavor eigenstate percentage for each mass eigenstate is shown through the amount of red $\left(\nu_{e}\right)$, green $\left(\nu_{\mu}\right)$, and blue $\left(\nu_{\tau}\right)$ in the line.

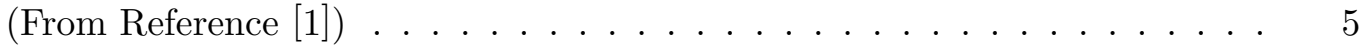

2.2 Mass hierarchy with one heavy sterile neutrino. The $\Delta m^{2}$ from LSND is incorporated. . . . . . . . . . . . . . . . . . . 9

$2.3 \quad$ Limits on mixing angle $\sin ^{2}(\theta)=\left|U_{e, 4}\right|^{2}$ from $\bar{\nu}_{e} \rightarrow \nu_{x}$ disappearance (left), on $\sin ^{2}(\theta)=\left|U_{\mu, 4}\right|^{2}$ from $\nu_{\mu} \rightarrow \nu_{x}$ disappearance (middle), and on $\sin ^{2}\left(2 \theta_{L S N D}\right) \approx$ $4\left|U_{e, 4} U_{\mu, 4}\right|^{2}$ (right) for $3+1$ models as a function of $\Delta m_{4,1}^{2}$. The allowed regions are shown in grey $(99 \%$ confidence level) and in red (90\% confidence level). The fit from the limits on the left and middle excludes the LSND decay-at-rest (DAR) data (shaded grey and red) on the right at 90\% confidence level (dashed blue) and 99\% confidence level (solid blue). atm $+\mathrm{NEV}+\mathrm{MB} 475$ stands for the sum of atmospheric oscillation measurement (atm) data, short-baseline experiments observing no evidence (NEV) data, and MiniBooNE data above $475 \mathrm{MeV}$ (MB475). (From Reference [2]) 11

2.4 Mass hierarchy with two heavy sterile neutrino. The $\Delta m^{2}$ from LSND is incorporated. . . . . . . . . . . . . . . . . . . . . 12 
2.5 Current global limits on disappearance searches. The 90\% confidence level limit from MiniBooNE simultaneous fit (solid black curve), MiniBooNE spectrum fit (dashed red curve), MiniBooNE only fit (dark grey), MINOS (hash green box), and CCFR/CDHS (light gray region) are shown. The predicted 90\% confidence level sensitivity of the MiniBooNE simultaneous fit (dotdashed blue curve) and MiniBooNE only fit (dashed cyan curve) is also shown. 13

3.1 Schematic view of the BNB to the SciBooNE and MiniBooNE detectors. . . 15

3.2 Schematic layout of the BNB. (From Reference 3$]$ ) . . . . . . . . . . 15

3.3 The BNB beryllium target. The top shows an exploded view of the compo\begin{tabular}{|c|}
\hline nents and the bottom shows the assembled configuration. The proton beam \\
\hline
\end{tabular}

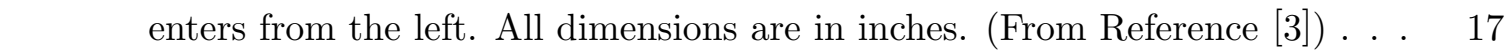

3.4 Schematic view of the BNB magnetic horn. The outer conductor of the horn is shown in grey and the inner conductor components of the horn are shown in dark green and blue. The plumbing associated with the water cooling system is also shown. (From Reference $3[$ ) . . . . . . . . . . . . . . 18

3.5 Schematic view of the entire SciBooNE detector. . . . . . . . . . . . . 20

3.6 Schematic drawing of a scintillator strip in three-dimensional view (a) and cross sectional view (b). All units are in $\mathrm{mm}$. . . . . . . . . . . . . . 21

3.7 Three-dimensional view of the SciBar and EC sub-detectors. The SciBar subdetector consists of alternating scintillator strips (shown in white), each instrumented with a green wavelength shifting fiber down its the center (shown in the green lateral exterior of SciBar), and read out by MA-PMTs (grey rectangle shown in the inset). The EC sub-detector consists of two planes of scintillating fibers covered with lead foil. The antineutrino beam is assumed to be incident from the left. . . . . . . . . . . . . . . . . . . . . . . . . . 22

3.8 Schematic diagram of the SciBar readout. . . . . . . . . . . . . . 23

3.9 Three-dimensional view of the MRD sub-detector. . . . . . . . . . . 25

3.10 Types of antineutrino interactions in SciBooNE and their associated detection signatures. . . . . . . . . . . . . . . . . . . 26

3.11 Schematic view of the MiniBooNE enclosure . . . . . . . . . . . . . . . 27 
3.12 Schematic view of the MiniBooNE detector. . . . . . . . . . . . 28

3.13 Photo of a MiniBoonE PMT. . . . . . . . . . . . . . . . . . . 29

3.14 Types of antineutrino interactions in MiniBooNE and their associated detection signatures. . . . . . . . . . . . . . . . . . . . . . . . . . . . 30

4.1 The cross section measurements of proton/Beryllium interactions with the Glauber model. The top left figure shows the total hadronic cross section for Beryllium in neutron/Beryllium interactions data (box), neutron/Beryllium interactions prediction (red circle), and proton/Beryllium interactions prediction (blue circle) within the allowed systematic uncertainties (red dash). The top right figure shows the total inelastic hadronic cross section for Beryllium in proton/Beryllium data (blue box) and parametrization (black line) within systematic uncertainties (red dash). The bottom middle figure shows the total quasielastic hadronic cross section for proton/Beryllium interactions with parametrization (black line). . . . . . . . . . . . . . 34

$4.2 \quad \pi^{-}$production cross section HARP data at $8.9 \mathrm{GeV} /$ c from proton-Beryllium collisions (red crosses) as a function of $\pi^{-}$momentum $\left(p_{\pi^{-}}\right)$in $\mathrm{GeV} / \mathrm{c}$ for different intervals of $\pi^{-}$angle $\left(\theta_{\pi^{-}}\right)$. The best fit SW parametrization (blue line) and 40 out of the 1000 spline fits to the HARP data (black lines) are also shown . . . . . . . . . . . . . . . . . . . . . . . . 36

$4.3 \quad \pi^{+}$production cross section HARP data at $8.9 \mathrm{GeV} / \mathrm{c}$ from proton-Beryllium collisions (red crosses) as a function of $\pi^{+}$momentum $\left(p_{\pi^{+}}\right)$in $\mathrm{GeV} / \mathrm{c}$ for different intervals of $\pi^{+}$angle $\left(\theta_{\pi^{+}}\right)$. The best fit SW parametrization (blue line) and 40 out of the 1000 spline fits to the HARP data (black crosses) are also shown . . . . . . . . . . . . . . . . . . . . . 37

4.4 Measurements of the azimuthal magnetic field within the horn. The points show the measured magnetic field, while the line shows the expected $1 / r$ dependence. The black vertical line indicates the outer radius of the horn. . 39 
4.5 The neutrino and antineutrino flux prediction as a function of true neutrino(antineutrino) energy, in antineutrino mode at the MiniBooNE and SciBooNE detectors. The $\bar{\nu}_{\mu}$ flux is represented by the solid line, the $\nu_{\mu}$ flux is represented by the dashed line, the $\bar{\nu}_{e}$ flux is represented by the dot-dashed line, and the $\nu_{e}$ flux is represented by the dotted line. . . . . . . . . . . 41

4.6 Previous world published data on neutrino CC cross sections. The CC cross sections are separated by interaction type and divided by neutrino energy and plotted as a function of neutrino energy. Charged current quasielastic interaction is shown in red. Charged current single pion interactions are shown in blue. Deep inelastic scattering interactions are shown in green. Total CC cross sections are shown in black. The colored points are the data with uncertainties and the colored solid lines are the default NUANCE simulation predictions before further tuning based on MiniBooNE analyses. $\quad 43$

4.7 Previous world published data on antineutrino CC cross sections. The CC cross sections are separated by interaction type and divided by antineutrino energy and plotted as a function of antineutrino energy. Charged current quasielastic interaction is shown in red. Charged current single pion interactions are shown in blue. Deep inelastic scattering interactions are shown in green. Total CC cross sections are shown in black. The colored points are the data with uncertainties and the colored solid lines are the default NUANCE simulation predictions before further tuning based on MiniBooNE analyses. 44

4.8 MiniBooNE muon neutrino CCQE cross section per neutron as a function of neutrino energy. In (a), shape errors are shown as shaded boxes along with the total errors as bars. In (b), a larger energy range is shown along with results from the LSND and NOMAD experiments. Also shown are predictions from the NUANCE simulation for an RFG model with $M_{A}=1.35 \mathrm{GeV} / \mathrm{c}^{2}$ and $\kappa=1.007$, for an RFG model with $M_{A}=1.03 \mathrm{GeV} / \mathrm{c}^{2}$ and $\kappa=1.000$, and for scattering from free nucleons with the world-average $M_{A}$ value. . . 46 
5.1 History of the accumulated number of protons on target (POT) for the entire SciBooNE data run. The first and third time periods correspond to the antineutrino mode runs used in the analysis. The solid blue line is the cumulative protons on target delivered and the dashed blue line is the cumulative protons on target collected for analysis. . . . . . . . . . . . . . . . . . 59

5.2 The number of charged current candidate events normalized to the number of protons on target as a function of time in SciBar (left) and in MRD (right). The first and third time periods for both figures correspond to the antineutrino mode running. . . . . . . . . . . . . . . . . . . . . . . . 59

5.3 The rate at which neutrinos and antineutrino events are collected during the length of the MiniBooNE run period. . . . . . . . . . . . . . . . . 60

5.4 Event display in top (X-Z plane) and side (Y-Z plane) views of a muon antineutrino charged current quasielastic candidate in SciBooNE antineutrino run data . . . . . . . . . . . . . . . . . . . . 61

5.5 Range to muon energy conversion table used to reconstruct $E_{\mu^{+}}^{S c i B a r}$ for SciBar

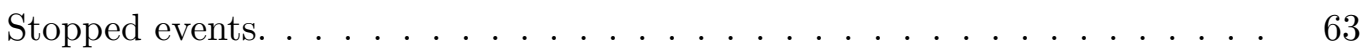

5.6 Event display in top (X-Z plane) and side (Y-Z plane) views of a SciBar Stopped event in antineutrino run data before (top) and after (bottom) track reconstruction. The blue line is the reconstructed candidate $\mu^{+}$track. . . . 65

5.7 Range to muon energy conversion table used to reconstruct $E_{\mu}^{M R D}$ for MRD Stopped events. . . . . . . . . . . . . . . . 66

5.8 Event display in top (X-Z plane) and side (Y-Z plane) views of a MRD Stopped event in antineutrino run data before (top) and after (bottom) SBMRD matching. In the top figure, the blue line is the reconstructed SciBar track and the dashed red line is the reconstructed MRD track. The long orange line in the bottom figure is the reconstructed candidate $\mu^{+}$track. . . $\quad 67$

$5.9 \quad$ Event display of a muon antineutrino charged current quasielastic candidate in MiniBoonE. . . . . . . . . . . . . . . . . . . . . . . . . . . . . 69 
5.10 SciBooNE reconstructed antineutrino energy resolution $\left(\frac{\text { reconstructed } E_{\nu}^{Q E}-\text { true } E_{\nu}}{\text { true } E_{\nu}}\right)$ for all and true CCQE selected MC events. The black curves show the Gaussian fits to the reconstructed antineutrino energy resolution. . . . . . . . . .

$5.112 \mathrm{D}$ histogram of true $E_{\nu}$ versus reconstructed $E_{\nu}^{Q E}$ for selected CCQE events (top) and selected non-CCQE events in MiniBooNE. The black line is at $\mathrm{y}=\mathrm{x}$. 74

5.12 Reconstructed antineutrino and neutrino energy $\left(E_{\nu}^{Q E}\right)$ distributions for selected RS and WS MiniBooNE events for different interaction types (CCQE, $\mathrm{CC} 1 \pi$, other) from MiniBooNE MC. Total events are represented by the solid line, CCQE interaction events are represented by the dashed line, CC1 $\pi$ interaction events are represented by the dot-dashed line, and all other interaction (CC multi- $\pi$ or NC) events are represented by the short-dashed line. . . . .

5.13 Reconstructed antineutrino and neutrino energy $\left(E_{\nu}^{Q E}\right)$ distributions for selected RS and WS SciBooNE events for different interaction types (CCQE, CC1 $\pi$, other) from SciBooNE MC. Total events are represented by the solid line, CCQE interaction events are represented by the dashed line, CC1 $\pi$ interaction events are represented by the dot-dashed line, and all other interaction (CC multi- $\pi$ or NC) events are represented by the short-dashed line. . . . . 76

5.14 Reconstructed antineutrino and neutrino energy $\left(E_{\nu}^{Q E}\right)$ distributions for selected RS and WS MiniBooNE events on different target types (hydrogen or carbon) from MiniBooNE MC. Total events are represented by the solid line, events with interaction on carbon are represented by the dashed line, and events with interaction on hydrogen are represented by the dot-dashed line. $\quad 78$

5.15 Reconstructed antineutrino and neutrino energy $\left(E_{\nu}^{Q E}\right)$ distributions for selected RS and WS SciBooNE events on different target types (hydrogen or carbon) from SciBooNE MC. Total events are represented by the solid line, events with interaction on carbon are represented by the dashed line, and events with interaction on hydrogen are represented by the dot-dashed line. $\quad 79$

5.16 Antineutrino and neutrino path lengths for MiniBooNE and SciBooNE events from point of production to interaction in detector, as predicted by the MC

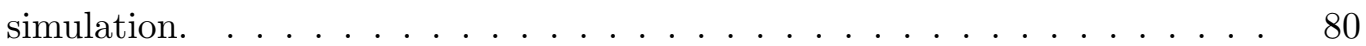


6.1 The MiniBooNE-SciBooNE flux error matrix (left) and its correlation (right) are shown as a function of reconstructed antineutrino energy bins with bin limits listed in Table 6.2 , The bin limits are the same for both MiniBooNE and SciBooNE with bins 1-21 for MiniBooNE and bins 22-42 for SciBooNE. 89

6.2 The ratio of neutrino cross section to antineutrino cross section $\left(\sigma^{\nu} / \sigma^{\bar{\nu}}\right)$ for the various nuclear models relative to neutrino cross section to antineutrino cross section $\left(\sigma^{\nu} / \sigma^{\bar{\nu}}\right)$ for the RFG model with CCQE $M_{A}=1.35 \mathrm{GeV} / \mathrm{c}^{2}$ and $\kappa=1.007$ as a function of (anti)neutrino energy. CCQE for the initial

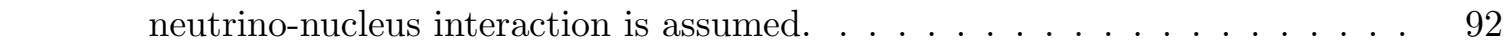

6.3 The MiniBooNE-SciBooNE neutrino interaction error matrix (left) and its correlation (right) are shown as a function of reconstructed antineutrino energy bins with bin limits listed in Table 6.2 , The bin limits are the same for both MiniBooNE and SciBooNE with bins 1-21 for MiniBooNE and bins $22-42$ for SciBooNE. . . . . . . . . . . . . . . . . . . . . . . . . 94

6.4 The SciBooNE detector error matrix (left) and its correlation (right) are shown as a function of SciBooNE reconstructed antineutrino energy bins \begin{tabular}{l|l|l|l} 
with bin limits listed in Table & 6.2 & The 21 SciBooNE antineutrino energy
\end{tabular} bins presented here have the exact same limits as the SciBooNE antineutrino energy bins in the MiniBooNE and SciBooNE error matrices for other uncertainties. . . . . . . . . . . . . . . . . . 96

6.5 The MiniBooNE detector error matrix (left) and its correlation (right) are shown as a function of MiniBooNE reconstructed antineutrino energy bins \begin{tabular}{l|l|l} 
with bin limits listed in Table & 6.2 & The 21 MiniBooNE antineutrino energy
\end{tabular} bins presented here have the exact same limits as the MiniBooNE antineutrino energy bins in the MiniBooNE and SciBooNE error matrices for other uncertainties. . . . . . . . . . . . . . . . . . . 97

6.6 The MiniBooNE-SciBooNE flux right-sign fractional error matrix (left) and flux wrong-sign fractional error matrix (right) are shown as a function of reconstructed antineutrino energy bins. Bins 1-21 are MiniBooNE and bins $22-42$ are SciBoonE. . . . . . . . . . . . . . . . . . . . . . . . . 103 
6.7 The MiniBooNE-SciBooNE neutrino interaction right-sign fractional error matrix (left) and neutrino interaction wrong-sign fractional error matrix (right) are shown as a function of reconstructed antineutrino energy bins. Bins 1-21 are MiniBooNE and bins 22-42 are SciBooNE. . . . . . . . . . . . 104

6.8 The SciBooNE detector right-sign fractional error matrix (left) and detector wrong-sign fractional error matrix (right) are shown as a function of reconstructed antineutrino energy bins. The 21 SciBooNE antineutrino energy bins presented here have the exact same limits as the SciBooNE antineutrino energy bins in the MiniBooNE and SciBooNE error matrices for other uncertainties. . . . . . . . . . . . . . . . . . 105

6.9 The MiniBooNE detector right-sign fractional error matrix (left) and detector wrong-sign fractional error matrix (right) are shown as a function of reconstructed antineutrino energy bins. The 21 MiniBooNE antineutrino energy bins presented here have the exact same limits as the MiniBooNE antineutrino energy bins in the MiniBooNE and SciBooNE error matrices for other uncertainties. . . . . . . . . . . . . . . . 106

6.10 The MiniBooNE-SciBooNE total error matrix (left) and its correlation (right) are shown as a function of reconstructed antineutrino energy bins. Bins 1-21 are MiniBoonE and bins 22-42 are SciBooNE. . . . . . . . . . . . . . . . . 107

6.11 The MiniBooNE $\sigma / \mu$ fractional uncertainties for flux (top left), neutrino interactions (top right), detector (bottom left), and total systematic and statistical (bottom right) as a function of reconstructed antineutrino energy in GeV. These are calculated from the diagonal elements of the error matrices so they do not capture any correlated error effects. . . . . . . . . . . . . . . 108

6.12 The SciBooNE $\sigma / \mu$ fractional uncertainties for flux (top left), neutrino interaction (top right), detector (bottom left), and total systematic and statistical (bottom right) as a function of reconstructed antineutrino energy in GeV. These are calculated from the diagonal elements of the error matrices so they do not capture any correlated error effects. . . . . . . . . . . . . . 109 
7.1 Two dimensional scatter plot of normalization variations of MiniBooNE and SciBooNE. Each point in the scatter plot represents a MiniBooNE and Sci-

BooNE fake data normalization (both normalized relative to default MC prediction). The x-axis is the MiniBooNE normalization (sum of all MB reconstructed antineutrino energy bins for one fake data relative to sum of all

\begin{tabular}{|l|}
\hline default MB reconstructed antineutrino energy bins) and the y-axis is the Sci- \\
\hline BooNE normalization (sum of all SB reconstructed antineutrino energy bins \\
\hline
\end{tabular}

for one fake data relative to sum of all default SB reconstructed antineutrino
energy bins). 100 scatter points are plotted and no oscillation signal is assumed.117

7.2 SciBooNE fake data value over MiniBooNE fake data value as a function of reconstructed antineutrino energy bins. Both the SciBooNE and MiniBooNE fake data values in each bin are normalized to default SciBooNE and Mini\begin{tabular}{|c|}
\hline BooNE MC prediction bin values, respectively. 100 histograms are plotted \\
\hline
\end{tabular}

7.3 Averaged exclusion limits at the $90 \%$ and $3-\sigma$ confidence levels and possible \begin{tabular}{|c|}
\hline oscillation signal region (left) and best fit $\Delta \chi^{2}$ points (right) for many fake \\
\hline
\end{tabular} data sets all with no oscillation signal assumed. The allowed oscillation \begin{tabular}{|c|}
\hline signal region is in light blue. The regions were produced assuming the $\Delta \chi^{2}$ \\
\hline
\end{tabular} distribution is a $\chi^{2}$ distribution with 2 DOF. . . . . . . . . . . 119

7.4 Fake data $\Delta \chi^{2}$ distribution with the blue line showing a $\chi^{2}$ distribution with 2 DOF (left) and fake data $\chi^{2}$ distribution with the blue line showing a $\chi^{2}$ distribution with 40 DOF right for many fake data sets and MC prediction

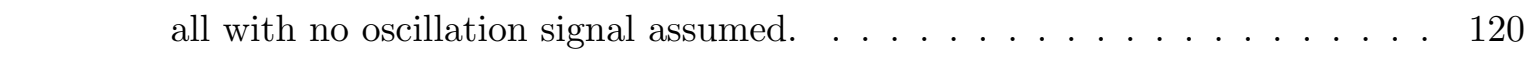

7.5 Averaged oscillation signal region and exclusion limits at the $90 \%$ and $3-\sigma$ \begin{tabular}{|c|}
\hline confidence levels (left) and best fit $\Delta \chi^{2}$ points (right) for many fake data sets \\
\hline all with oscillation signal of $\Delta m^{2}=1.0 \mathrm{eV}^{2}$ and $\sin ^{2} 2 \theta=0.2$ assumed. The \\
\hline
\end{tabular} allowed oscillation signal region is in light blue. The regions were produced

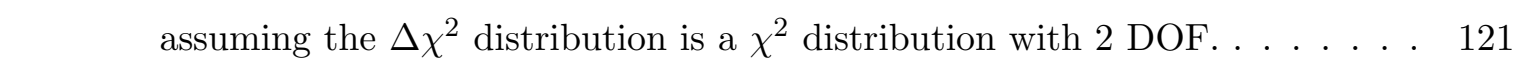


7.6 Fake data $\Delta \chi^{2}$ distribution with the blue line showing a $\chi^{2}$ distribution with 2 DOF (left) and fake data $\chi^{2}$ distribution with the blue line showing a $\chi^{2}$ distribution with 40 DOF right for many fake data sets and MC prediction all with oscillation signal of $\Delta m^{2}=1.0 \mathrm{eV}^{2}$ and $\sin ^{2} 2 \theta=0.2$ assumed. . . . 122

7.7 $\quad$ Averaged oscillation signal region and exclusion limits at the $90 \%$ and $3-\sigma$ confidence levels (left) and best fit $\Delta \chi^{2}$ points (right) for many fake data sets all with oscillation signal of $\Delta m^{2}=1.0 \mathrm{eV}^{2}$ and $\sin ^{2} 2 \theta=0.4$ assumed. The allowed oscillation signal region is a light blue. The regions were produced assuming the $\Delta \chi^{2}$ distribution is a $\chi^{2}$ distribution with 2 DOF. . . . . . . . 123

7.8 $\quad$ Fake data $\Delta \chi^{2}$ distribution with the blue line showing a $\chi^{2}$ distribution with 2 DOF (left) and fake data $\chi^{2}$ distribution with the blue line showing a $\chi^{2}$ distribution with 40 DOF right for many fake data sets and MC prediction all with oscillation signal of $\Delta m^{2}=1.0 \mathrm{eV}^{2}$ and $\sin ^{2} 2 \theta=0.4$ assumed. . . . 124

7.9 Averaged exclusion limits at the $90 \%$ and $3-\sigma$ confidence levels and possible oscillation signal region (left) and best fit $\Delta \chi^{2}$ points (right) for many fake data sets all with oscillation signal of $\Delta m^{2}=10.0 \mathrm{eV}^{2}$ and $\sin ^{2} 2 \theta=0.2$ assumed. The allowed oscillation signal region is a light blue. The regions were produced assuming the $\Delta \chi^{2}$ distribution is a $\chi^{2}$ distribution with 2 DOF. 125

7.10 Fake data $\Delta \chi^{2}$ distribution with the blue line showing a $\chi^{2}$ distribution with 2 DOF (left) and fake data $\chi^{2}$ distribution with the blue line showing a $\chi^{2}$ distribution with 40 DOF right for many fake data sets and MC prediction all with oscillation signal of $\Delta m^{2}=10.0 \mathrm{eV}^{2}$ and $\sin ^{2} 2 \theta=0.2$ assumed. . . 126

7.11 MC predictions of the deficit expected in the antineutrino energy spectrum due to several different $\sin ^{2} 2 \theta$ and $\Delta m^{2}$ oscillation signals normalized to default MC prediction (no oscillation signal) at the SciBooNE and MiniBooNE detectors. The black line at 1 represents the default MC prediction. The red histogram represents an oscillation signal with $\Delta m^{2}=1.0 \mathrm{eV}^{2}$ and $\sin ^{2} 2 \theta=0.2$. The green histogram represents an oscillation signal with $\Delta m^{2}=1.0 \mathrm{eV}^{2}$ and $\sin ^{2} 2 \theta=0.4$. The blue histogram represents an oscillation signal with $\Delta m^{2}=10.0 \mathrm{eV}^{2}$ and $\sin ^{2} 2 \theta=0.2 . \ldots \ldots \ldots 127$ 
7.12 Fake data exclusion limits at the $90 \%$ confidence level assuming no oscillation signal for $\Delta m^{2}$ between $0.01 \mathrm{eV}^{2}$ and $100 \mathrm{eV}^{2}$ (left) and $\Delta m^{2}$ between 100 $\mathrm{eV}^{2}$ and $10000 \mathrm{eV}^{2}$ (right). The regions were produced assuming the $\Delta \chi^{2}$ distribution is a $\chi^{2}$ distribution with 2 DOF. . . . . . . . . . . . . 128

7.13 Reconstructed antineutrino energy $\left(E_{\nu}^{Q E}\right)$ distribution for data events (crosses), compared to MC predictions (solid lines), for MiniBooNE and SciBooNE. Vertical error bars on data are the statistical uncertainties. Shaded error bands around the MC predictions are the systematic uncertainties computed as the square roots of the diagonals of the total systematic error matrix. . . 133

7.14 90\% CL exclusion region (solid line) and best fit point for the joint MiniBooNE, SciBooNE $\bar{\nu}_{\mu}$ disappearance analysis in linear scale for $\sin ^{2} 2 \theta$ and $\Delta m^{2}$. Also shown is the 90\% CL result from the 2009 MiniBooNE disappearance analysis (dashed line) and the CCFR experiment (dot-dashed line). The expected 90\% CL sensitivity band from fake data studies is also shown (shaded region); $1 \sigma(68 \%)$ of fake data tests, where the fake data had statistical and systematic fluctuations but no oscillation signal, had 90\% CL limit curves in this shaded region. . . . . . . . . . . . . . . . . . . . 134

$7.1590 \%$ CL exclusion region (solid line) and best fit point for the joint MiniBooNE, SciBooNE $\bar{\nu}_{\mu}$ disappearance analysis in log scale for $\sin ^{2} 2 \theta$ and $\Delta m^{2}$. Also shown is the 90\% CL result from the 2009 MiniBooNE disappearance analysis (dashed line) and the CCFR experiment (dot-dashed line). The expected 90\% CL sensitivity band from fake data studies is also shown (shaded region); $1 \sigma(68 \%)$ of fake data tests, where the fake data had statistical and systematic fluctuations but no oscillation signal, had 90\% CL limit curves in this shaded region. . . . . . . . . . . . . . . . . . 135 
$7.1690 \%$ CL exclusion region (solid line) and best fit point for the MiniBooNEonly $\bar{\nu}_{\mu}$ disappearance analysis. Also shown is the $90 \%$ CL result from the 2009 MiniBooNE disappearance analysis (dashed line) and the CCFR experiment (dot-dashed line). The expected 90\% CL sensitivity band from fake data studies is also shown (shaded region); $1 \sigma(68 \%)$ of fake data tests, where the fake data had statistical and systematic fluctuations but no oscillation signal, had 90\% CL limit curves in this shaded region. . . . . . . . . . . . . 136

7.17 The ratio, with full error bars (systematic and statistical), between data and default $\mathrm{MC}$ as a function of reconstructed antineutrino energy $\left(E_{\nu}^{Q E}\right)$. The ratio of best fit signal MC to default MC is also shown (dashed line). The best fit results from the joint analysis were used to generate the signal MC. The shaded regions are the $1 \sigma$ band from fake data with statistical and systematic fluctuations but no oscillation signal. . . . . . . . . . . . . 137

7.18 The double ratio (Eq. 7.7), with full error bars (systematic and statistical), as a function of reconstructed antineutrino energy $\left(E_{\nu}^{Q E}\right)$. Some of the MiniBooNE and SciBooNE uncertainties cancel in this double ratio. The double ratio where the MiniBooNE and SciBooNE signal MC based on the best fit results from the joint analysis are used in placed of data is also shown (dashed line). The shaded region is the $1 \sigma$ band from fake data with statistical and systematic fluctuations but no oscillation signal. . . . . . . . . . . . . . 138 


\section{List of Tables}

$2.1 \quad$ Upper limits on the direct mass measurements of the neutrino flavor eigenstates. 4

$4.1 \quad$ SW fit parameters for $\pi^{+}$and $\pi^{-}$production from HARP and BNL E910 data. 35

$4.2 \quad$ Feynman scaling fit parameters for $K^{+}$production. . . . . . . . . . . . . . 38

$4.3 \quad$ SW fit parameters for $K^{0}$ production from BNL E910 and KEK data. . . . 38

5.1 Summary of data run periods for SciBooNE and MiniBooNE. . . . . . . . . 56

$5.2 \quad$ SciBooNE beam quality checks and fractions of the total number of protons on target that fail each check. . . . . . . . . . . . . . . . 58

$5.3 \quad$ List of cuts for the MiniBooNE CCQE $\mu^{+}$event selection. . . . . . . . . . . 70

5.4 The standard deviations $(\sigma)$ of the Gaussian fits to the reconstructed antineutrino energy resolution distributions for selected MC MiniBooNE and SciBooNE CCQE and total events. . . . . . . . . . . . . . . . . 73

$5.5 \quad$ MC predictions for the number of selected events by neutrino and interaction type for both MiniBooNE and SciBooNE. . . . . . . . . . . . . . . 77

6.1 Summary of antineutrino beam flux and neutrino interaction parameters for MC simulation with its associated systematic uncertainties. . . . . . . . . . 93

6.2 Reconstructed antineutrino energy bin limits for MiniBooNE and SciBooNE. 101

7.1 Observed event counts for each MiniBooNE and SciBooNE reconstructed antineutrino energy bin, for data, MC predictions, and uncertainties. . . . . 130 


\section{Acknowledgments}

First, I would like to express my deepest gratitude to my PhD advisor, Professor Michael H. Shaevitz. Thank you for all those years of wisdom, knowledge, and guidance.

Second, I would like to thank all the members of the SciBooNE collaboration. In particular, I would like to thank Dr. Camillo Mariani, for providing his knowledge, advice, and expertise and for the patience to allow me to work through my mistakes. I would also like to give a special thanks to Yasuhiro Nakajima for his constant and unwavering help with so many essential details about the SciBooNE experiment. Thank you Teppei Katori, Kendall Mahn, Katsuki Hiraide, Yoshi Kurimoto, Hideyuki Takei, Aaron Hanson, Morgan Wascko, Sam Zeller, Michel Sorel, Hide Tanaka-san, Masashi Yokoyama-san, Tsuyoshi Nakaya-san, and everyone else in SciBooNE for making my summer at Fermilab so memorable and for your constructive comments throughout my graduate student years.

I would also like to thank all the people in the MiniBooNE collaboration. A special thanks to Dr. Warren Huelsnitz, for working patiently alongside me through this complex and difficult analysis.

I would like to thank the Columbia neutrino group and the Nevis Laboratory staff and colleagues for creating such a warm, fun, and stimulating work environment. Thank you Dr. Leslie Camilleri, Matt Toups, Arthur Franke, Georgia Karagiorgi, Bill Seligman, Rachel Carr, David Kaleko, Tim Andeen, Grace Ho, Amy Garwood, and everyone else for your knowledge and assistance.

To the many other friends I made throughout my graduate student years, thank you. Your friendship and support during thick and thin mean so much to me.

Finally, I wish to thank both my father and mother, Yuan Chih Cheng and Hsiao Lung Ma, for their inspiration, wisdom, and support. Your encouragement gave me the strength to achieve my goals. I would not be the person I am today without you. 


\section{Chapter 1}

\section{Introduction}

Neutrino masses have been one of the only additions to the wildly successful Standard Model of particle physics since its construction during the 1960s and 1970s. Neutrino masses were implemented in the early 1990s due to the observation of solar and atmospheric neutrino oscillations. Neutrino oscillations is the phenomenon when one flavor of neutrino changes into another flavor of neutrino as the neutrino propagates through a distance. This change can only occur if there are small differences in the mass of the neutrinos, which implies that neutrinos possess mass.

Most experimental measurements are consistent with the theory of three neutrino generations, but the LSND and MiniBooNE experiments suggest the existence of additional "sterile" neutrinos that are only accessible through neutrino oscillations. A neutrino (or antineutrino) disappearance search is one of the methods available to detect the presence of neutrino oscillations into sterile neutrinos. In a disappearance search, a known neutrino source of a specific flavor, $\nu_{\alpha}$, is allowed to oscillate into another (possibly unobserved) flavor of neutrino, $\nu_{\beta}$, hence giving the impression of a "disappearance" in the original neutrino flavor $\nu_{\alpha}$. This thesis describes a search for muon antineutrino disappearance oscillations consistent with the LSND and MiniBooNE suggestions of sterile neutrinos, using both the MiniBooNE and SciBooNE detectors in conjunction. The use of both MiniBooNE and SciBooNE detectors in conjunction cancels out much of the antineutrino flux and neutrino interaction uncertainties in the analysis, since they are common to both detectors and correlated. The SciBooNE detector acts as a near detector that measures the antineutrino 
flux and constrains its uncertainties while the MiniBooNE acts as a far detector that measures the disappearance oscillation. This cancellation of uncertainties results in a better sensitivity.

Chapter 2 describes the basic properties of the neutrino (antineutrino), introduces neutrino (antineutrino) oscillations, and explains the need for neutrino (antineutrino) disappearance in the search for sterile neutrinos. Chapter 3 describes the neutrino beamline, which provides the antineutrino source, and the two detectors, MiniBooNE and SciBooNE, which observe the antineutrino source. Chapter 4 explains how the beamline, neutrino and antineutrino interactions, and detection of neutrinos and antineutrinos are simulated.

The next three chapters of the thesis contain the describe the analysis associated with the thesis topic. Chapter 5 describes the reconstruction and selection of antineutrino events in both detectors necessary for the analysis. Chapter 6 describes the systematic and statistical uncertainties of the analysis associated with the beamline, detection, reconstruction, and selection. Finally, Chapter 7 details the combined antineutrino disappearance analysis using selected events in MiniBooNE and SciBooNE, including the tests of robustness, consistency, and sensitivity for the analysis. The thesis concludes with a summary of the results of the analysis and future prospects. 
CHAPTER 2. NEUTRINOS, NEUTRINO OSCILLATIONS, AND STERILE

\section{Chapter 2}

\section{Neutrinos, Neutrino Oscillations, and Sterile Neutrinos}

\subsection{Introduction to Neutrinos}

Neutrinos and their antiparticles, antineutrinos, are electrically neutral elementary particles with spin 1/2. They obey Fermi-Dirac statistics and are classified as leptons in the Standard Model of particle physics. Neutrinos were first postulated by Wolfgang Pauli in 1930 to explain the carrier of missing energy in the decay of the neutron.

Neutrinos and antineutrinos interact only through the weak interaction and gravity. There are three neutrino (antineutrino) weak interaction eigenstates, also known as flavor eigenstates: the electron neutrino $\nu_{e}$ (electron antineutrino $\bar{\nu}_{e}$ ), the muon neutrino $\nu_{\mu}$ (muon antineutrino $\left.\bar{\nu}_{\mu}\right)$, and the tau neutrino $\nu_{\tau}\left(\right.$ tau antineutrino $\left.\bar{\nu}_{\tau}\right)$. There are only three flavor eigenstates because the decay of the $Z$ boson constrains the number of neutrinos from its decay width 44.

Direct mass measurements of the three neutrino flavor eigenstates have been performed by measuring the kinematics of particle decays. No absolute mass has yet been measured but upper limits have been placed on all three neutrino flavor eigenstates as shown in Table 2.1. 
CHAPTER 2. NEUTRINOS, NEUTRINO OSCILLATIONS, AND STERILE NEUTRINOS

\begin{tabular}{|c|c|c|}
\hline Neutrino Flavor & Mass & Experiment \\
\hline$\nu_{e}$ & $<2.3 \mathrm{eV} / \mathrm{c}^{2}(95 \% \mathrm{CL})$ & Tritium $\beta$ Decay $|5|$ \\
\hline$\nu_{\mu}$ & $<0.17 \mathrm{MeV} / \mathrm{c}^{2}(90 \% \mathrm{CL})$ & Pion Decay 6 \\
\hline$\nu_{\tau}$ & $<18.2 \mathrm{MeV} / \mathrm{c}^{2}(95 \% \mathrm{CL})$ & Tau Decay 7 \\
\hline
\end{tabular}

Table 2.1: Upper limits on the direct mass measurements of the neutrino flavor eigenstates.

\subsection{Neutrino Oscillations}

Neutrino oscillations occur because the mass eigenstates are different from the flavor eigenstates. Neutrinos are always produced as one of the flavor eigenstates. Since neutrinos propagate through vacuum as a mixture of mass eigenstates that have different DeBroglie wavelengths, each neutrino, produced in a flavor eigenstate can change flavor in the propagation. The constructive and destructive interference between the different mass eigenstates as the neutrino propagates through vacuum causes the sinusoidal neutrino oscillation effect.

Currently, there are three known and universally accepted neutrino mass eigenstates, designated as $\nu_{1}, \nu_{2}$, and $\nu_{3}$ with masses $m_{1}, m_{2}$, and $m_{3}$, respectively. Though the absolute masses are unknown, the splitting between the square of the masses for $\nu_{1}$ and $\nu_{2}$, or $\Delta m_{12}=$ $m_{2}^{2}-m_{1}^{2}$, and the splitting between the square of the masses for $\nu_{2}$ and $\nu_{3}$, or $\Delta m_{23}=$ $m_{3}^{2}-m_{2}^{2}$, are known from neutrino oscillations. This leaves two possible configurations of neutrino masses, or "mass hierarchies". In the "normal" hierarchy, the mass of $\nu_{3}$ is much larger than the masses of $\nu_{1}$ and $\nu_{2}$. In the "inverted" hierarchy, the masses of $\nu_{1}$ and $\nu_{2}$ are much larger than the mass of $\nu_{3}$. Figure 2.1 shows the masses of $\nu_{1}, \nu_{2}$, and $\nu_{3}$, with their relative flavor eigenstate composition, for both mass hierarchies.

Each neutrino mass eigenstate can be expressed as superposition of the neutrino flavor eigenstates. The neutrino mass eigenstate are then defined as:

$$
\left|\nu_{i}\right\rangle=\sum_{\alpha}^{3} U_{i, \alpha}\left|\nu_{\alpha}\right\rangle
$$

where $\left|\nu_{i}\right\rangle$ is the mass eigenstate $i$ of the neutrino (with $i=1,2,3$ corresponding to the three known mass eigenstates), $\left|\nu_{\alpha}\right\rangle$ is the flavor eigenstate (with $\alpha=\mathrm{e}, \mu, \tau$ ), and $U_{i, \alpha}$ are elements of the mixing matrix $U$ between flavor and mass eigenstates. 


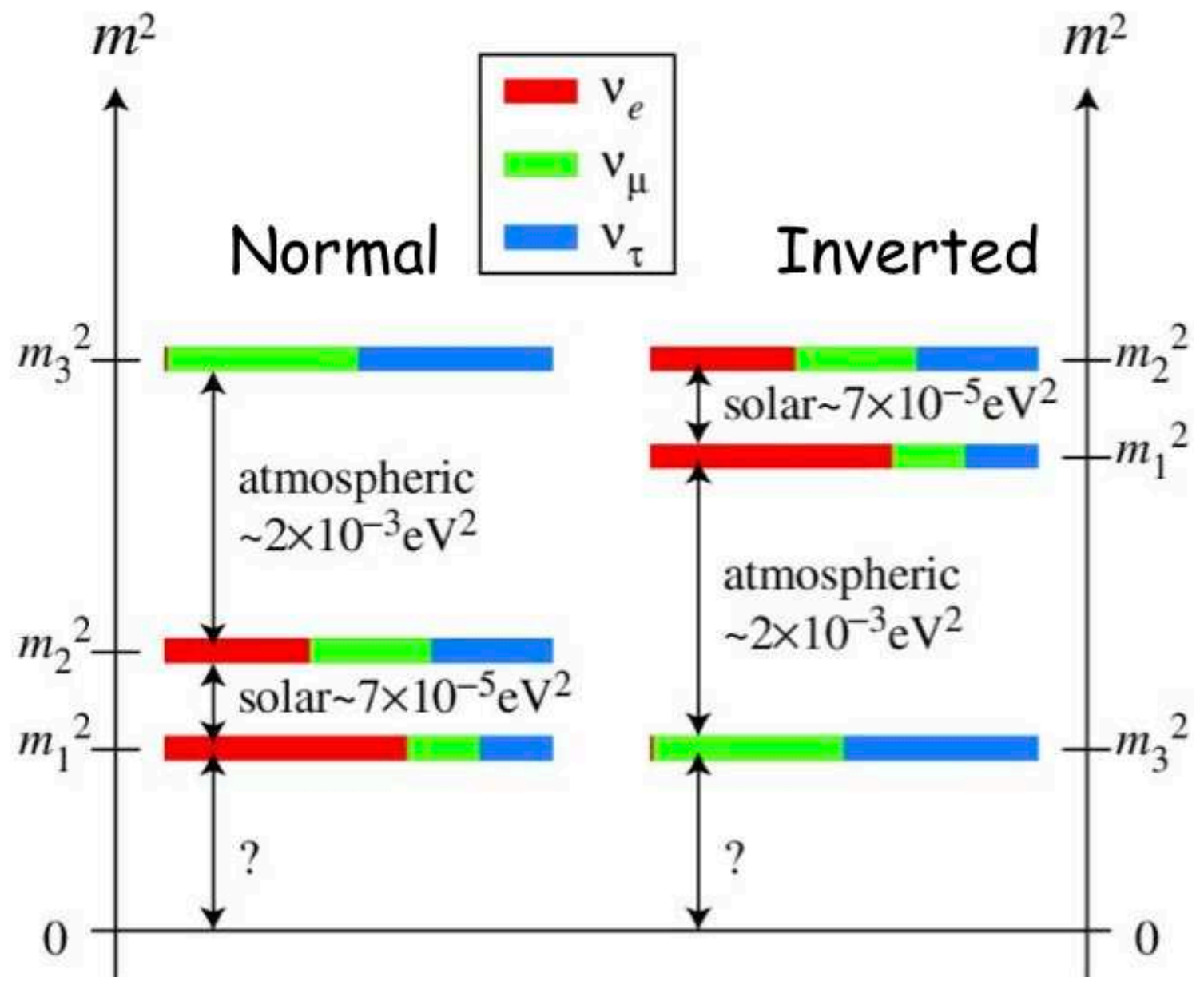

Figure 2.1: The neutrino mass eigenstates in the normal and inverted neutrino mass hierarchies. The flavor eigenstate percentage for each mass eigenstate is shown through the amount of red $\left(\nu_{e}\right)$, green $\left(\nu_{\mu}\right)$, and blue $\left(\nu_{\tau}\right)$ in the line. (From Reference [1])

$U$, the mixing matrix between the flavor and mass eigenstates, can be factorized and expressed as three matrices:

$$
U=\left(\begin{array}{ccc}
1 & 0 & 0 \\
0 & c\left(\theta_{23}\right) & s\left(\theta_{23}\right) \\
0 & -s\left(\theta_{23}\right) & c\left(\theta_{23}\right)
\end{array}\right)\left(\begin{array}{ccc}
c\left(\theta_{13}\right) & 0 & s\left(\theta_{13}\right) e^{-i \delta} \\
0 & 1 & 0 \\
-s\left(\theta_{13}\right) e^{-i \delta} & 0 & c\left(\theta_{13}\right)
\end{array}\right)\left(\begin{array}{ccc}
c\left(\theta_{12}\right) & s\left(\theta_{12}\right) & 0 \\
-s\left(\theta_{12}\right) & c\left(\theta_{12}\right) & 0 \\
0 & 0 & 1
\end{array}\right)
$$

where $c(\omega)$ is $\cos (\omega), s(\omega)$ is $\sin (\omega), \theta_{12}, \theta_{13}, \theta_{23}$ are the neutrino mixing angles, and $\delta$ is a $\mathrm{CP}$ violating phase. Generally, it would be impossible to measure each of the neu- 
trino mixing angles individually since the oscillation effects from the different mixing angles would interfere with each other, but nature has been kind. Since $\Delta m_{23}$ is much larger than $\Delta m_{12}$ (2 order of magnitude difference), $\theta_{13}$ is relatively small, and $\theta_{23}$ is close to $45^{\circ}$, the oscillations from the different mixing angles effectively decouple from each other. Oscillations from one mixing angle produces high frequency and small amplitude oscillations on top of another mixing angle oscillation with low frequency and large amplitude. Therefore, neutrino oscillation experiments can designed to be mainly sensitive to one neutrino mixing angle.

There are two general methods used to search for neutrino oscillations: appearance and disappearance. In appearance searches, a known neutrino source of a specific flavor $\nu_{\alpha}$ is generated (or occurs in nature) and the goal is to observe the oscillated neutrinos of another flavor, $\nu_{\beta}$, a distance away. Since $\nu_{\alpha} \rightarrow \nu_{\beta}$ is not equal to $\bar{\nu}_{\alpha} \rightarrow \bar{\nu}_{\beta}$ due to CP violation, appearance searches have the advantage that it can detect $\mathrm{CP}$ violation if both $\nu_{\alpha} \rightarrow \nu_{\beta}$ and $\bar{\nu}_{\alpha} \rightarrow \bar{\nu}_{\beta}$ can be measured. In disappearance searches, a known neutrino source of a specific flavor $\nu_{\alpha}$ is generated (or occurs in nature) and the goal is to observe a deficit in neutrinos of the same flavor, $\nu_{\alpha}$, a distance away. Assuming CPT invariance, $\nu_{\alpha} \rightarrow \nu_{\alpha}$ is equivalent to $\bar{\nu}_{\alpha} \rightarrow \bar{\nu}_{\alpha}$ and disappearance searches have the advantage that data can be combined from both $\nu_{\alpha} \rightarrow \nu_{\alpha}$ and $\bar{\nu}_{\alpha} \rightarrow \bar{\nu}_{\alpha}$ to achieve better sensitivity for the desired neutrino oscillation.

To better understand neutrino mixing and knowing that each neutrino mixing angle can be effectively isolated for measurement, consider only the two neutrino mixing case between $\nu_{\mu}$ and $\nu_{\tau}$ determined by the leftmost block of $U . \nu_{\mu}$ at time $t$ can be expressed as a superposition of $\nu_{2}$ and $\nu_{3}$ :

$$
\left|\nu_{\mu}(t)\right\rangle=\cos \left(\theta_{23}\right) e^{-i E_{2} t}\left|\nu_{2}\right\rangle+\sin \left(\theta_{23}\right) e^{-i E_{3} t}\left|\nu_{3}\right\rangle
$$

where $E_{i}$ is the energy of neutrino $\nu_{i}$ in the laboratory frame. Neutrinos are extremely relativistic so the following approximations are made:

$$
\begin{gathered}
t \approx l \\
E_{i}=\operatorname{sqrt}\left(p_{i}^{2}+m_{i}^{2}\right) \approx p_{i}+\frac{m_{i}^{2}}{2 p_{i}}
\end{gathered}
$$


where $p_{i}$ is the momentum of $\nu_{i}$ and $l$ is the distance of propagation.

Hence, the probability to observe a $\nu_{\mu}$ after it has traveled a distance $L$ in $\mathrm{km}$ with energy $E$ in $\mathrm{GeV}$ is:

$$
P\left(\nu_{\mu} \rightarrow \nu_{\mu}\right)=\left|\left\langle\nu_{\mu} \mid \nu_{\mu}(t)\right\rangle\right|^{2}=1-\sin ^{2} 2 \theta_{23} \sin ^{2} \frac{1.27 \Delta m_{23}^{2} L}{E}
$$

where $\Delta m_{23}=m_{3}^{2}-m_{2}^{2}$ is the difference between the mass squared of mass eigenstate $3, m_{3}$, and the mass squared of mass eigenstate $2, m_{2}$. This is the $\nu_{\mu}$ disappearance probability, since there is a probability that the original $\nu_{\mu}$ would not be observed. Instead the $\nu_{\mu}$ would be observed as a $\nu_{e}$, leading to the $\nu_{e}$ appearance probability:

$$
P\left(\nu_{\mu} \rightarrow \nu_{e}\right)=\sin ^{2} 2 \theta_{23} \sin ^{2} \frac{1.27 \Delta m_{23}^{2} L}{E}
$$

The form of Equation 2.6 and Equation 2.7 are true for any two-state neutrino mixing and are coupled together due to the sum of probability being equal to unity. The probability for oscillation depends only on the mixing angle, the splitting between the square of the mass eigenstates, the neutrino energy and travel distance. The mixing angle and the splitting between the square of the mass eigenstates are fixed by nature, but an experiment can be judiciously designed to take advantage of $L$ and $E$ to maximize appearance and disappearance. Notice that if the neutrinos have no mass, the neutrino oscillation would be zero.

\subsubsection{Neutrino Measurements}

Over the years, there have been many neutrino oscillation experiments measuring atmospheric neutrinos, solar neutrinos, nuclear reactor neutrinos, and accelerator neutrinos. Four distinct $\Delta m^{2}$ regions are observed: solar, atmospheric, reactor, and LSND.

\subsubsection{Solar Neutrino Oscillations}

The sun produces $\nu_{e}$ through its fusion process. The solar neutrino oscillation was discovered by measuring the $\nu_{e} \rightarrow \nu_{x}$ oscillation from the sun (Homestake [8, GALLEX [9], GNO [10, SAGE [11], SNO [12], SK [13], and Borexino [14]) and verified through the reactor neutrino experiment KamLAND 15. From solar neutrino experiments, $\theta_{12} \approx 31^{\circ}$ and $\Delta m_{12}^{2} \approx$ $7.6 \times 10^{-5} \mathrm{eV}^{2} \underline{16}$. 


\subsubsection{Atmospheric Neutrino Oscillations}

Cosmic rays striking the atmosphere produces charged pions that decay into $\nu_{\mu} \mathrm{s}$ and $\nu_{e} \mathrm{~s}$. The atmospheric neutrino oscillation was discovered by the SK experiment 17 through the measurement of the $\nu_{\mu} \rightarrow \nu_{x}$ oscillation using $\nu_{\mu}$ s produced in the atmosphere. The result was later confirmed by accelerator experiments K2K [18 and MINOS [19]. From atmospheric neutrino experiments, $\theta_{23} \approx 42^{\circ}$ and $\Delta m_{23}^{2} \approx 2.4 \times 10^{-3} \mathrm{eV}^{2}$ 16.

\subsubsection{Reactor Neutrino Oscillations}

Nuclear fission reactors produce a high flux of $\bar{\nu}_{e}$ from the decay of fission products. The reactor neutrino oscillation was discovered by measuring the $\bar{\nu}_{e} \rightarrow \nu_{x}$ oscillation from reactors (Double Chooz [20], Daya Bay 21], and RENO [22]). From reactor neutrino experiments, $\theta_{13} \approx 16^{\circ}$ and $\Delta m_{13}^{2} \approx 2.4 \times 10^{-3} \mathrm{eV}^{2}$.

\subsection{LSND Observation and Sterile Neutrinos}

The LSND experiment observed an excess of $\bar{\nu}_{e}$ in a $\bar{\nu}_{\mu}$ beam [23] at an energy $E$ and distance $L$ not consistent with previous measurements of neutrino mixing angles and $\Delta m^{2}$ values in the three flavor eigenstate and three mass eigenstate model. The excess $\bar{\nu}_{e}$ corresponded to another oscillation with a $\Delta m^{2}$ of around $1 \mathrm{eV}^{2}$. Since both $\Delta m_{12}^{2}$ and $\Delta m_{23}^{2}$ have been measured (and with $\Delta m_{13}^{2}$ inferred from $\Delta m_{12}^{2}$ and $\Delta m_{23}^{2}$ ) and have values much less than $1 \mathrm{eV}^{2}$, there was no freedom in the model to accommodate a large $\Delta m^{2}$. The large $\Delta m^{2}$ indicates a fourth generation of neutrinos but since $Z$ boson decays constrain the number of neutrino flavors to three, this was not possible and an inconsistency arose. One solution to this inconsistency is to introduce one or more "sterile" neutrino states (with the simplest case being one) that do not do not couple to the weak interaction. Each of the new sterile neutrino flavor eigenstates is defined as:

$$
\left|\nu_{s}\right\rangle=\sum_{i}^{3+N} U_{\alpha, i}^{*}\left|\nu_{i}\right\rangle
$$

where there are $3+N$ mass eigenstates, the 3 original mass eigenstates and $N$ sterile mass eigenstates. The original $3 \times 3$ mixing matrix $U$ would be expanded to $(3+N) \times(3+N)$. 
Figure 2.2 shows the new mass hierarchy with one new sterile neutrino.

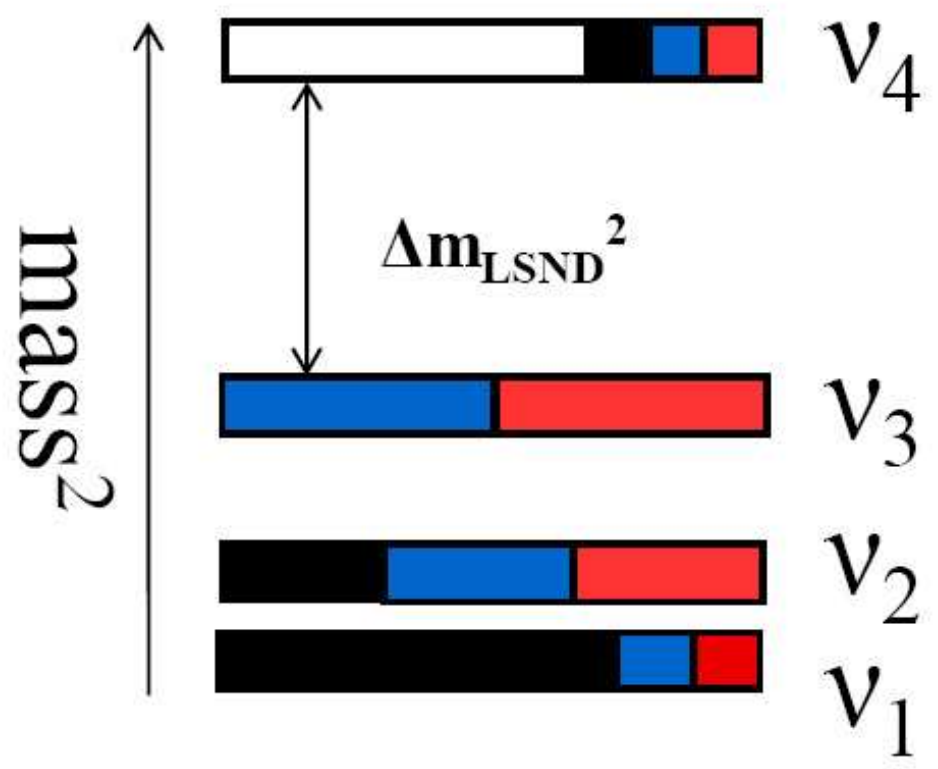

Figure 2.2: Mass hierarchy with one heavy sterile neutrino. The $\Delta m^{2}$ from LSND is incorporated.

Since the LSND $\Delta m^{2}$ splitting is much larger than the other $\Delta m^{2}$ splittings, the heavier sterile neutrino state would effectively mix with the other three mass eigenstates as if they were just one mass eigenstate to create the desired two-state neutrino oscillation and explain the excess of $\bar{\nu}_{e}$ in a $\bar{\nu}_{\mu}$ beam.

The MiniBooNE experiment was designed to test the LSND result. In 2007, the MiniBooNE experiment 24] excluded a two neutrino $\nu_{\mu} \rightarrow \nu_{e}$ appearance-only oscillation (98\% CL) as an explanation for the LSND excess, assuming CPT invariance $\left(P_{\bar{\nu}_{\mu} \rightarrow \bar{\nu}_{e}}=P_{\nu_{\mu} \rightarrow \nu_{e}}\right)$. However, in 2010, the MiniBooNE experiment 25] observed a hint for a two antineutrino $\bar{\nu}_{\mu} \rightarrow \bar{\nu}_{e}$ appearance-only oscillation consistent with the LSND measurement. In 2012, a combined $\nu_{\mu} \rightarrow \nu_{e}$ and $\bar{\nu}_{\mu} \rightarrow \bar{\nu}_{e}$ appearance-only oscillation measurement (assuming CPT conservation) by the MiniBooNE experiment observed a combined excess of $\nu_{e}$ and $\bar{\nu}_{e}$ in their $\nu_{\mu}$ and $\bar{\nu}_{\mu}$ beam at the 3.8- $\sigma$ level [26]. 
CHAPTER 2. NEUTRINOS, NEUTRINO OSCILLATIONS, AND STERILE

NEUTRINOS

\subsection{Antineutrino Disappearance}

Both neutrino and antineutrino disappearance searches provide complementary channels to neutrino and antineutrino appearance searches for additional oscillations. To better understand the relationship between appearance and disappearance searches, consider the $3+1$ sterile neutrino model. Assume that the sterile neutrino mass eigenstate, $m_{4}$, is much heavier than the 3 Standard Model mass eigenstates, so that the approximations $\Delta m_{12}^{2}=\Delta m_{13}^{2}=\Delta m_{23}^{2}=0$ and $\Delta m_{14}^{2}=\Delta m_{24}^{2}=\Delta m_{34}^{2}$ can be made. The oscillation probabilities are then

$$
P\left(\nu_{\alpha} \rightarrow \nu_{\beta \neq \alpha}\right)=4\left|U_{\alpha, 4}\right|^{2}\left|U_{\beta, 4}\right|^{2} \sin ^{2} \frac{1.27 \Delta m_{41}^{2} L}{E}
$$

and

$$
P\left(\nu_{\alpha} \rightarrow \nu_{\alpha}\right)=1-4\left|U_{\alpha, 4}\right|^{2}\left(1-\left|U_{\alpha, 4}\right|^{2}\right) \sin ^{2} \frac{1.27 \Delta m_{41}^{2} L}{E}
$$

where $\nu_{\alpha}$ and $\nu_{\beta}$ are the 3 Standard Model flavor eigenstates. From Equation 2.9, the probability of $\nu_{e}$ appearance from a $\nu_{\mu}$ beam, $P\left(\nu_{\mu} \rightarrow \nu_{e}\right)$, is proportional to $\left|U_{e 4}\right|^{2}\left|U_{\mu 4}\right|^{2}$. $\left|U_{e 4}\right|^{2}$ and $\left|U_{\mu 4}\right|^{2}$ can be constrained by $\nu_{e}$ and $\nu_{\mu}$ disappearance $\left(P\left(\nu_{e} \rightarrow \nu_{e}\right)\right.$ and $\left.P\left(\nu_{\mu} \rightarrow \nu_{\mu}\right)\right)$, respectively. This sets a one-to-one relation between the appearance and disappearance probabilities. Therefore, disappearance searches are complementary to appearance searches.

Assuming CPT invariance $\left(P_{\bar{\nu}_{\mu} \rightarrow \bar{\nu}_{\mu}}=P_{\nu_{\mu} \rightarrow \nu_{\mu}}\right)$, antineutrino disappearance searches are equivalent to neutrino disappearance searches (neutrino appearance searches are almost equivalent to antineutrino appearance searches with the difference coming from $\mathrm{CP}$ violation) and data from both types of disappearance searches can be combined. Disappearance searches are also directly sensitive to oscillations to sterile neutrino since disappearance searches can observe the deficit of neutrinos as the neutrinos oscillate into the sterile neutrino state.

Previous disappearance searches include $\bar{\nu}_{e} \rightarrow \nu_{x}$, where $\nu_{x}$ is a neutrino state other than $\bar{\nu}_{e}$, and $\nu_{\mu} \rightarrow \nu_{x}$, where $\nu_{x}$ a neutrino state other than $\nu_{\mu}$. Both types of disappearance searches did not observe evidence for sterile neutrino oscillation and their combined data have already excluded $3+1$ sterile neutrino models consistent with the observed LSND oscillation. Figure 2.3 2] shows the exclusion region of $3+1$ models, including the observed LSND oscillation region, using global data $27 ; 24 ; 28 ; 29 ; 30 ; 31 ; 32 ; 33$. 

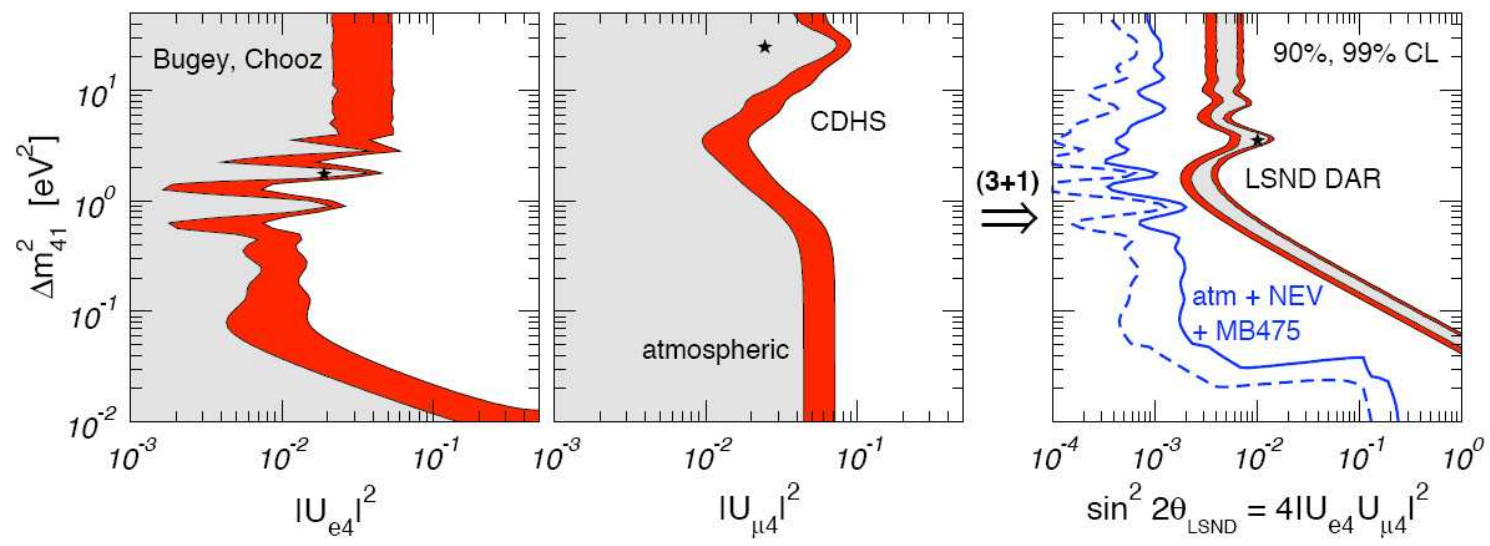

Figure 2.3: Limits on mixing angle $\sin ^{2}(\theta)=\left|U_{e, 4}\right|^{2}$ from $\bar{\nu}_{e} \rightarrow \nu_{x}$ disappearance (left), on $\sin ^{2}(\theta)=\left|U_{\mu, 4}\right|^{2}$ from $\nu_{\mu} \rightarrow \nu_{x}$ disappearance (middle), and on $\sin ^{2}\left(2 \theta_{L S N D}\right) \approx 4\left|U_{e, 4} U_{\mu, 4}\right|^{2}$ (right) for $3+1$ models as a function of $\Delta m_{4,1}^{2}$. The allowed regions are shown in grey (99\% confidence level) and in red (90\% confidence level). The fit from the limits on the left and middle excludes the LSND decay-at-rest (DAR) data (shaded grey and red) on the right at 90\% confidence level (dashed blue) and 99\% confidence level (solid blue). atm $+\mathrm{NEV}+\mathrm{MB} 475$ stands for the sum of atmospheric oscillation measurement (atm) data, short-baseline experiments observing no evidence (NEV) data, and MiniBooNE data above $475 \mathrm{MeV}$ (MB475). (From Reference 2])

$3+2$ sterile neutrino models, as shown in Figure 2.4, allows better compatibility between the LSND oscillation and previous disappearance searches by increasing the number of free parameters, though some inconsistencies remain. The oscillation probabilities are

$$
\begin{aligned}
P\left(\nu_{\alpha} \rightarrow \nu_{\beta \neq \alpha}\right)= & 4\left|U_{\alpha, 4}\right|^{2}\left|U_{\beta, 4}\right|^{2} \sin ^{2} x_{41}+4\left|U_{\alpha, 5}\right|^{2}\left|U_{\beta, 5}\right|^{2} \sin ^{2} x_{51} \\
& +8\left|U_{\alpha, 4}\right|\left|U_{\beta, 4}\right|\left|U_{\alpha, 5}\right|\left|U_{\beta, 5}\right| \sin x_{41} \sin x_{51} \cos \left(x_{54}-\phi_{54}\right)
\end{aligned}
$$

and

$$
\begin{aligned}
P\left(\nu_{\alpha} \rightarrow \nu_{\alpha}\right)= & 1-4\left[\left(1-\left|U_{\alpha, 4}\right|^{2}-\left|U_{\alpha, 5}\right|^{2}\right)\left(\left|U_{\alpha, 4}\right|^{2} \sin ^{2} x_{41}+\left|U_{\alpha, 5}\right|^{2} \sin ^{2} x_{41}\right)\right. \\
& \left.+\left|U_{\alpha, 4}\right|^{2}\left|U_{\alpha, 5}\right|^{2} \sin ^{2} x_{54}\right]
\end{aligned}
$$

where $\nu_{\alpha}$ and $\nu_{\beta}$ are the 3 Standard Model flavor eigenstates, $x_{i j}=\frac{1.27 \Delta m_{i j}^{2} L}{E}$, and $\phi_{54}=$ $\arg \left(U_{\alpha, 5} U_{\beta, 5}^{*} U_{\alpha, 4}^{*} U_{\beta, 5}\right)$. The inclusion of the additional parameters allow more freedom in 
reconciling the LSND oscillation and previous disappearance searches, by allowing no oneto-one relation between appearance and disappearance probabilities.

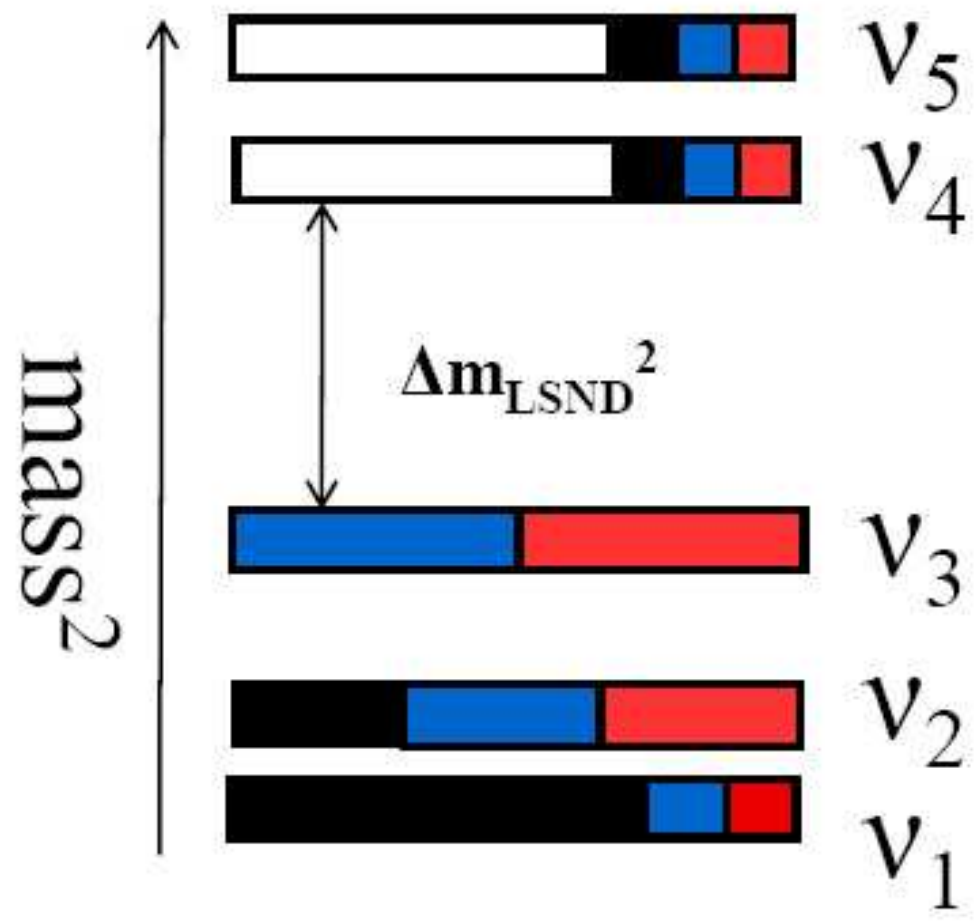

Figure 2.4: Mass hierarchy with two heavy sterile neutrino. The $\Delta m^{2}$ from LSND is incorporated.

Higher numbers of sterile neutrinos in $3+N$ sterile neutrino models fit all the observed data well, but also introduces the issue of additional complexity and parameters. Furthermore, there exist exotic models to try to explain the LSND oscillation including sterile neutrinos in extra dimensions [34, decaying sterile neutrinos [35], and CPT violation 36.

Figure 2.5 shows the current global limits on disappearance searches. Additional and more sensitive disappearance searches are necessary and important to detect whether sterile neutrinos actually exist and to determine which, if any, of the proposed $3+N$ sterile neutrino models are correct. This analysis, an antineutrino disappearance search of $\bar{\nu}_{\mu} \rightarrow \bar{\nu}_{x}$ where $\bar{\nu}_{x}$ is not the $\bar{\nu}_{\mu}$, is a step in that direction and will attempt to further resolve these outstanding issues with a measurement more sensitive than any previously obtained. 


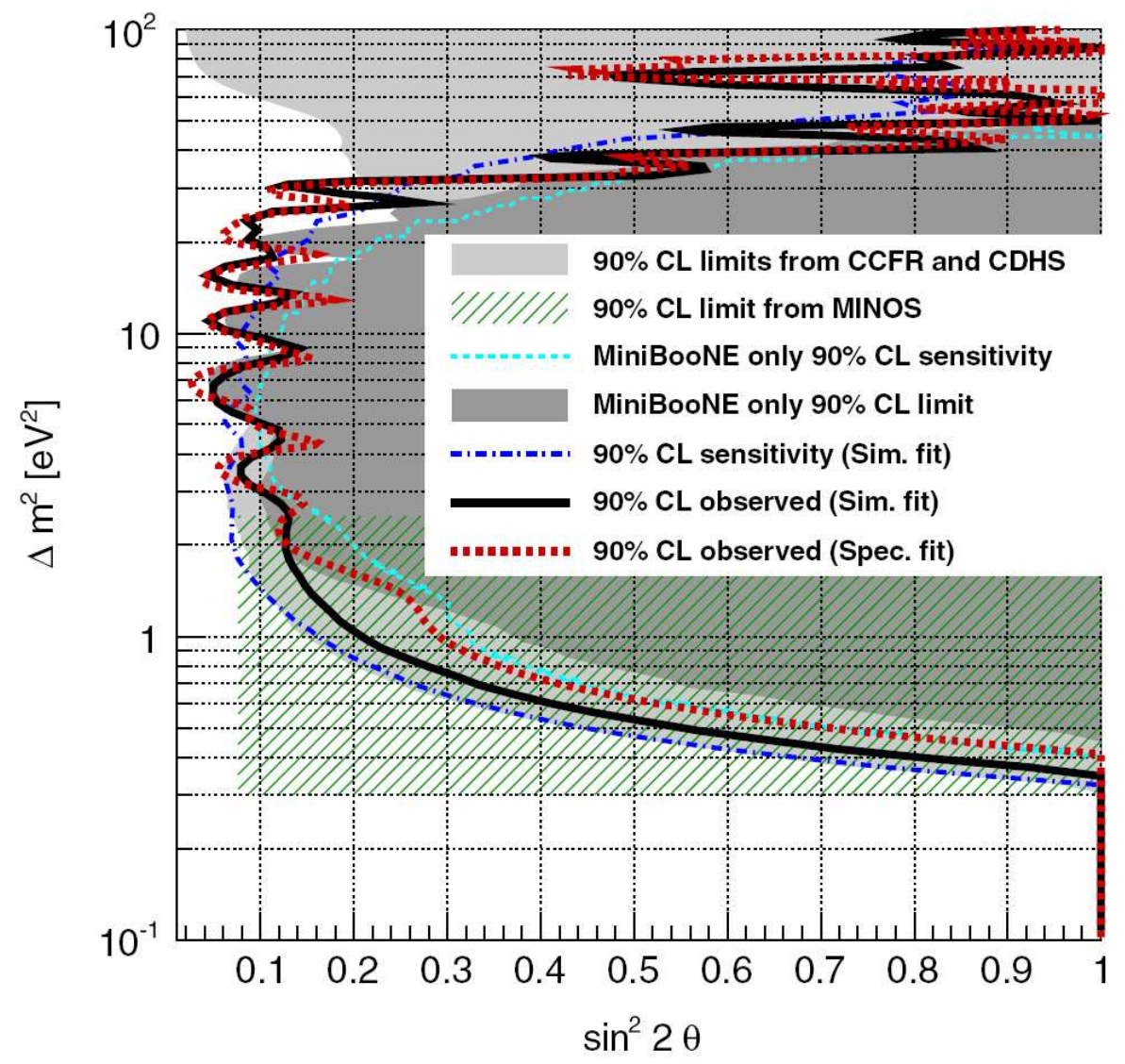

Figure 2.5: Current global limits on disappearance searches. The $90 \%$ confidence level limit from MiniBooNE simultaneous fit 37 (solid black curve), MiniBooNE spectrum fit 37. (dashed red curve), MiniBooNE only fit 38 (dark grey), MINOS 39 (hash green box), and CCFR 40/CDHS 30 (light gray region) are shown. The predicted $90 \%$ confidence level sensitivity of the MiniBooNE simultaneous fit (dot-dashed blue curve) and MiniBooNE only fit (dashed cyan curve) is also shown. (From Reference [37]) 


\section{Chapter 3}

\section{SciBooNE and MiniBooNE Experiments}

The purpose of this chapter is to explain the technical details of the experimental apparatus and setup. The details on the experimental apparatus and setup are required to understand the data being collected and how it is associated with the antineutrinos. These details also help one understand the relevant uncertainties that arises in the analysis. The production, propagation, and detection of the antineutrino beam as it relates to the experimental apparatus and setup is also described.

The chapter is organized in the order of antineutrino production to antineutrino detection. The Booster Neutrino Beamine (BNB) that produces and delivers the antineutrinos to the SciBooNE and MiniBooNE detectors is first described. The SciBooNE detector, the first of the two detectors downstream of the antineutrino beam, is then described. Finally, the MiniBooNE detector, the second of the two detectors downstream of the antineutrino beam, is described. A schematic view of the BNB to both detectors is shown in Figure 3.1.

\subsection{The Booster Neutrino Beamline (BNB)}

The Booster Neutrino Beamline (BNB) is located at the Fermi National Accelerator Laboratory (Fermilab) in Batavia, Illinois and provides either a high flux of neutrinos or a high flux of antineutrinos for the MiniBooNE and SciBooNE experiments. The BNB will 


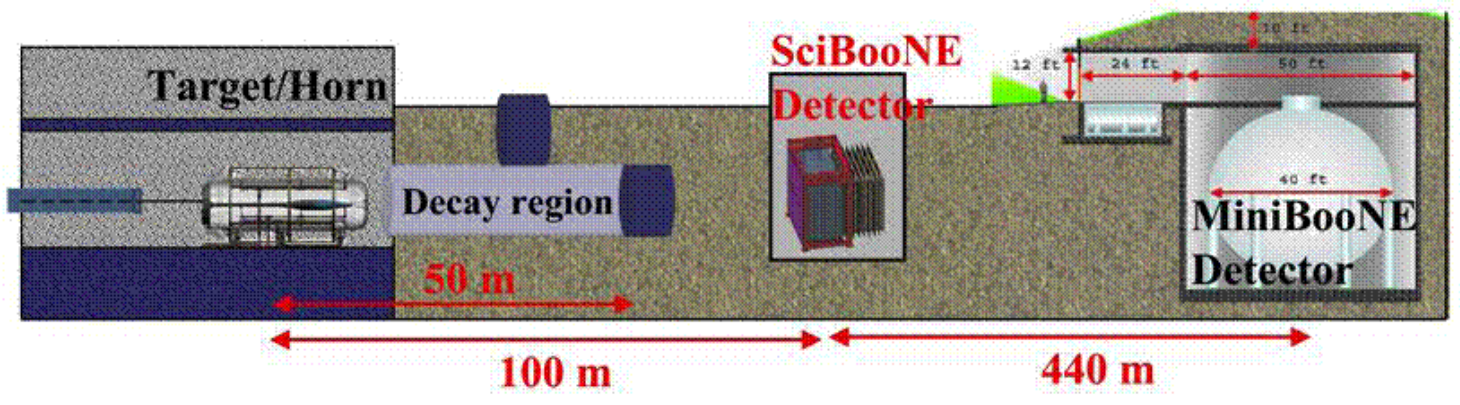

Figure 3.1: Schematic view of the BNB to the SciBooNE and MiniBooNE detectors.

continue to be used in the future to provide neutrino and antineutrino beams for the MicroBooNE experiment. The major components of the BNB, to be described in the order of production in this section, is presented in Figure 3.2 .

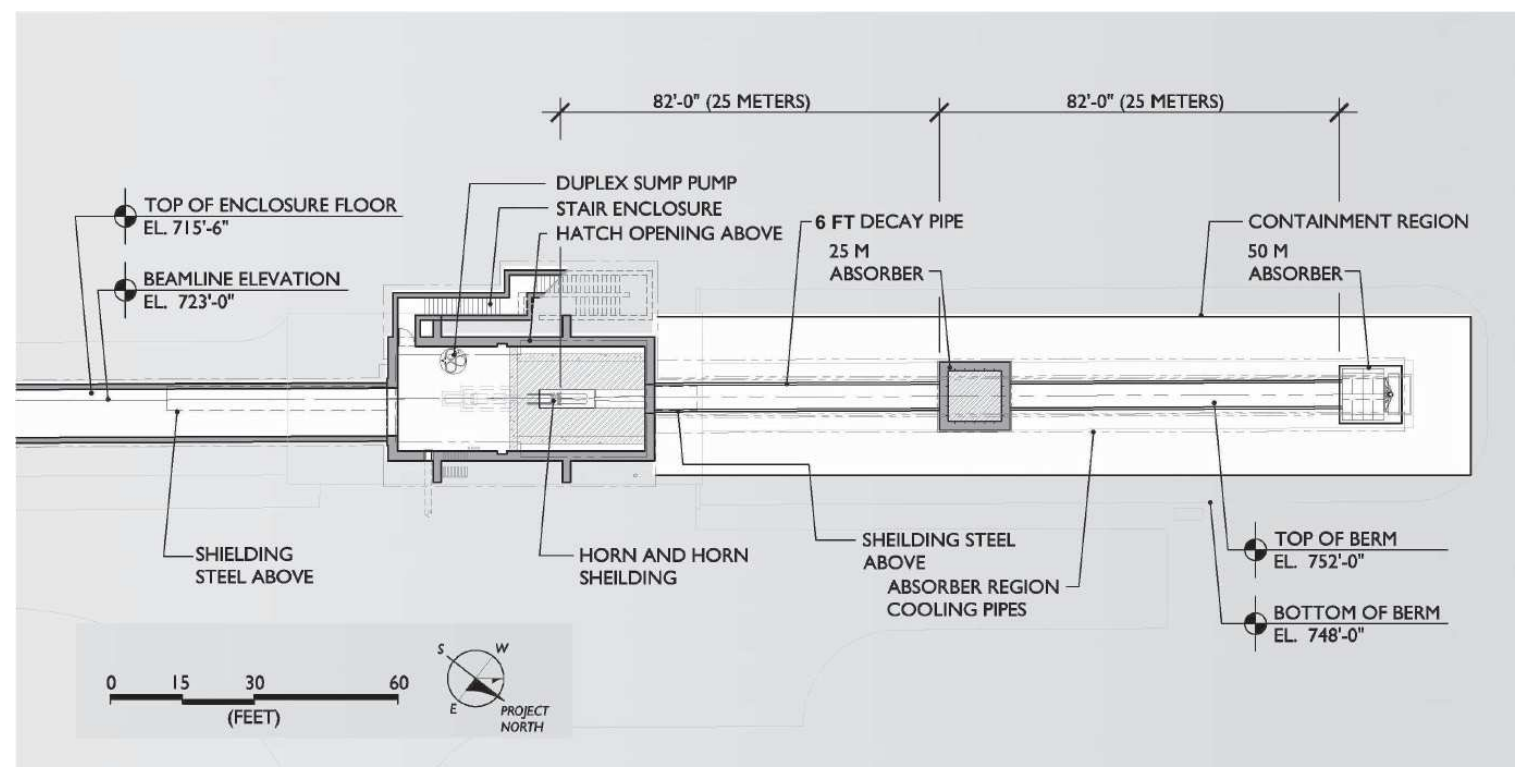

Figure 3.2: Schematic layout of the BNB. (From Reference [3])

The first step in producing a neutrino or antineutrino beam is to accelerate protons. For the $\mathrm{BNB}$, three accelerators in series accelerate protons up to $8 \mathrm{GeV}$ kinetic energy (8.9 $\mathrm{GeV} / \mathrm{c}$ momentum). $\mathrm{H}_{2}$ gas is converted into $\mathrm{H}^{-}$ions using a Cockcroft-Walton generator and accelerated up to $750 \mathrm{keV}$. The $\mathrm{H}^{-}$ions are then accelerated using alternating electro- 
magnetic fields in a linear accelerator up to $400 \mathrm{MeV}$. A thin carbon foil strips the electrons away, leaving only protons which enter the Booster synchrotron. The Booster synchrotron finally accelerates the protons up to $8 \mathrm{GeV}$ kinetic energy. The protons are accelerated in groups called beam spills. A typical beam spill contains $4 \times 10^{12}$ protons over a period of 1.6 $\mu$ s and proton spills can be sent down the beamline at a maximum rate of $5 \mathrm{~Hz}$ (typically 2-4 Hz). The nature of the RF acceleration technique results in 81 smaller bunches within each beam spill, each 6 ns wide and separated by 19 ns.

The $8 \mathrm{GeV}$ kinetic energy protons are directed onto a beryllium target producing the mesons that will decay into the desired neutrinos or antineutrinos. Before hitting the target, the protons pass through a series of beam position monitors and toroids, which measure the properties of the proton beam. The total number of protons on target (POT) as well as the position and spread of the beam on the target are measured. There are several toroids that are calibrated every year and agree within $2 \%$ tolerance. The protons then collide with the beryllium target, which produces the desired mesons. The beryllium target is composed of a cylindrical enclosure $71 \mathrm{~cm}$ long with a diameter of $1 \mathrm{~cm}$ that contains seven cylindrical slugs of beryllium aligned in succession along their radial axes and each supported by three fins within the enclosure. Air is circulated within the enclosure since the colliding protons generate a large amount of heat. A schematic of the BNB target and its setup is shown in Figure 3.3. The beryllium target has 1.7 interaction lengths. A beryllium target is used because beryllium has low $\mathrm{Z}$ so the interaction length is large relative to the radiation length and the colliding protons do no lose energy before colliding.

The beryllium target is surrounded by a magnetic focusing horn. The purpose of the magnetic focusing horn is to focus one sign of charged mesons and defocus the others. There are two modes of operation for the magnetic horn corresponding to two BNB running modes known as neutrino mode and antineutrino mode. In neutrino mode, the magnetic horn is set so that positively charged mesons such as $\pi^{+}$and $K^{+}$are focused in the forward beamline direction and negatively charged mesons such as $\pi^{-}$and $K^{-}$are de-focused away from the beamline direction. In antineutrino mode, the opposite is true and the magnetic horn is set so that negatively charged mesons such as $\pi^{-}$and $K^{-}$are focused in the forward beamline direction and positively charged mesons such as $\pi^{+}$and $K^{+}$are de-focused away from the 

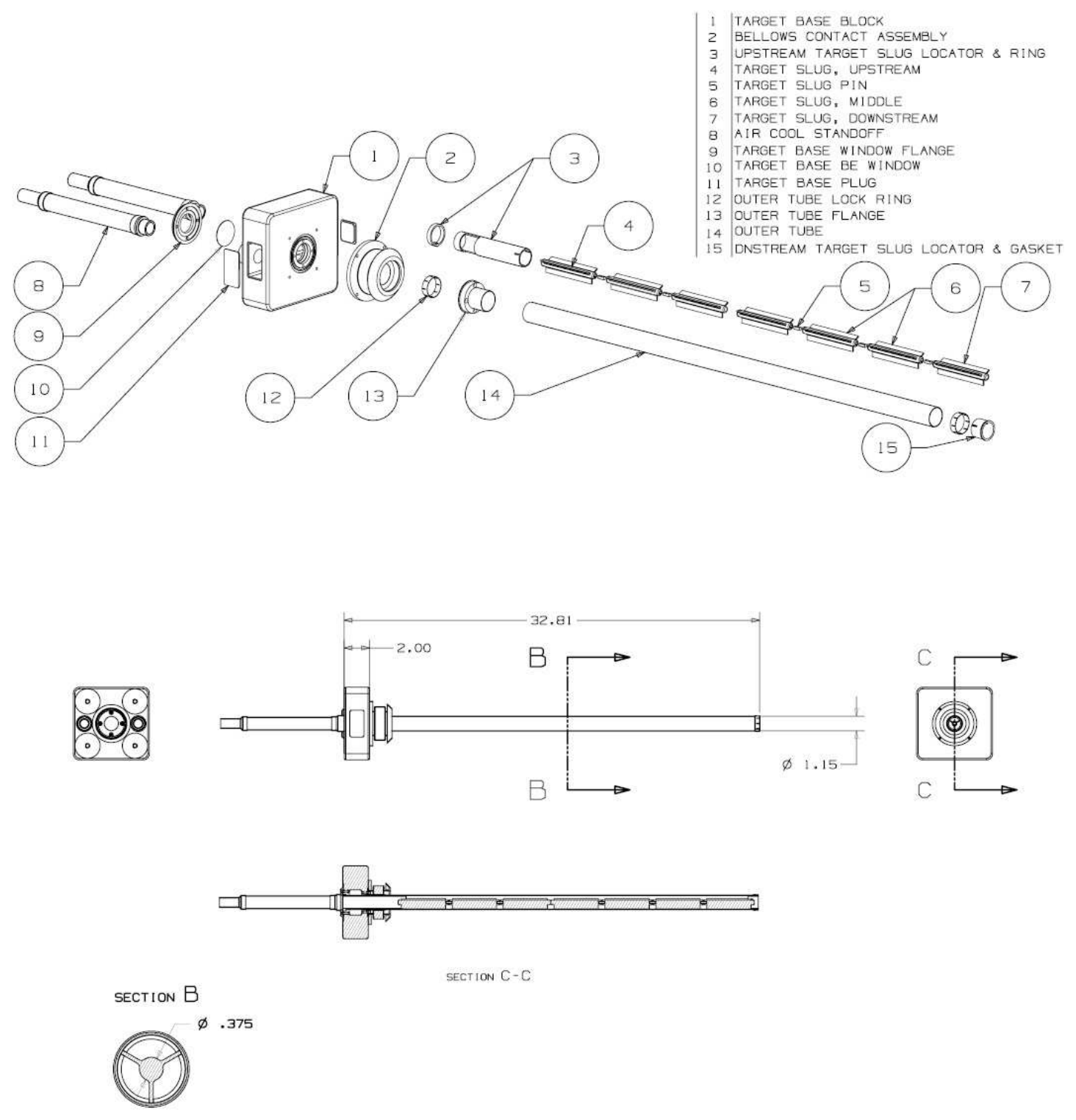

Figure 3.3: The BNB beryllium target. The top shows an exploded view of the components and the bottom shows the assembled configuration. The proton beam enters from the left. All dimensions are in inches. (From Reference [3])

beamline direction. The eventual result after the selected mesons decay is that the beam will either have an enhanced flux of muon neutrinos (neutrino mode) or muon antineutrinos (antineutrino mode). 
For this analysis, only the antineutrino mode configuration data and Monte Carlo simulation will be used. The predicted antineutrino fluxes at the MiniBooNE and SciBooNE locations are shown in Figure 4.5 in Chapter 4. In antineutrino mode running, the flux of antineutrinos in the beam will be referred to as the right-sign (RS) flux and the flux of neutrinos in the beam will be referred to as the wrong-sign (WS) flux. The WS flux comes from decayed $\pi^{+}$and $K^{+}$that were not de-focused by the magnetic horn in antineutrino mode running. These two designations are used because antineutrinos are the signal in this analysis and neutrinos are a background.

The magnetic focusing horn is constructed from aluminum and current flows between the inner and outer surfaces of the horn to produce the magnetic field used for focusing and de-focusing mesons. The current in the horn is pulsed in time with the proton beam spill. A schematic of the magnetic focusing horn is shown in Figure 3.4 .

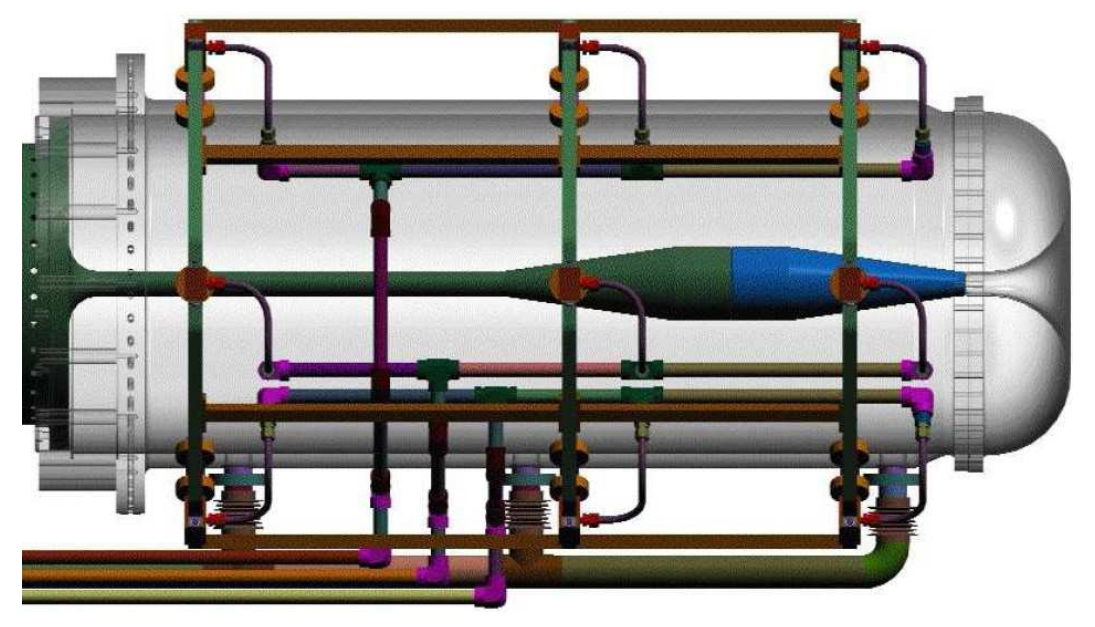

Figure 3.4: Schematic view of the BNB magnetic horn. The outer conductor of the horn is shown in grey and the inner conductor components of the horn are shown in dark green and blue. The plumbing associated with the water cooling system is also shown. (From Reference [3])

After focusing the negatively charged mesons decay into the desired antineutrino beam. The focused mesons pass through a $60 \mathrm{~cm}$ diameter cylindrical collimator and decay in flight along a cylindrical $50 \mathrm{~m}$ long, $182 \mathrm{~cm}$ diameter tunnel filled with air. The mesons, 
composed mainly of $\pi^{-}$in antineutrino mode, decay primarily into $\mu^{-}$and $\bar{\nu}_{\mu}$ in the decay tunnel (though there are smaller contributions from $\pi^{+}, K^{ \pm}$decays in beam). Since the $\pi^{-}$ are highly boosted in the forward beamline direction, the resulting $\mu^{-}$and $\bar{\nu}_{\mu}$ travel also in the forward beamline direction along the decay tunnel. The beam dump, a $3.8 \mathrm{~m}$ thick steel and concrete region located at the end of the decay tunnel, absorb the protons, muons, and all of the undecayed mesons in the beam and leave only the antineutrino beam going forward towards the MiniBooNE and SciBooNE detectors. Further details on the beamline and flux predictions are given in Reference 3 .

\subsection{The SciBooNE and MiniBooNE Detectors}

The SciBooNE [41] and MiniBooNE 42 detectors are located $100 \mathrm{~m}$ and $541 \mathrm{~m}$, respectively, downstream of the antineutrino production target. The two detectors use different detection methods as SciBooNE is a discrete tracking scintillator detector and MiniBooNE is an oil Cherenkov detector. The two detectors are also of different shapes and masses. The SciBooNE detector is cubical in shape with a total mass of 60 tons and fiducial antineutrino target mass of 10.6 tons. The MiniBooNE detector is spherical in shape with a total mass of 1000 tons and fiducial antineutrino target mass of 450 tons.

\subsection{The SciBooNE Detector}

SciBooNE is comprised of three sub-detectors (in order from upstream to downstream): a fully active and finely segmented scintillator tracker (SciBar), an electromagnetic calorimeter (EC), and a muon range detector (MRD). Figure 3.5 shows the schematic view of the entire SciBooNE detector.

\subsubsection{The SciBar Sub-detector}

The SciBar sub-detector is a fully active and finely segmented scintillator tracker that serves as the primary interaction target for the antineutrino beam. It is used to reconstruct the neutrino-nucleus and antineutrino-nucleus interaction vertices and detect charged particles produced by these neutrino and antineutrino interactions. Since SciBar is a finely segmented 


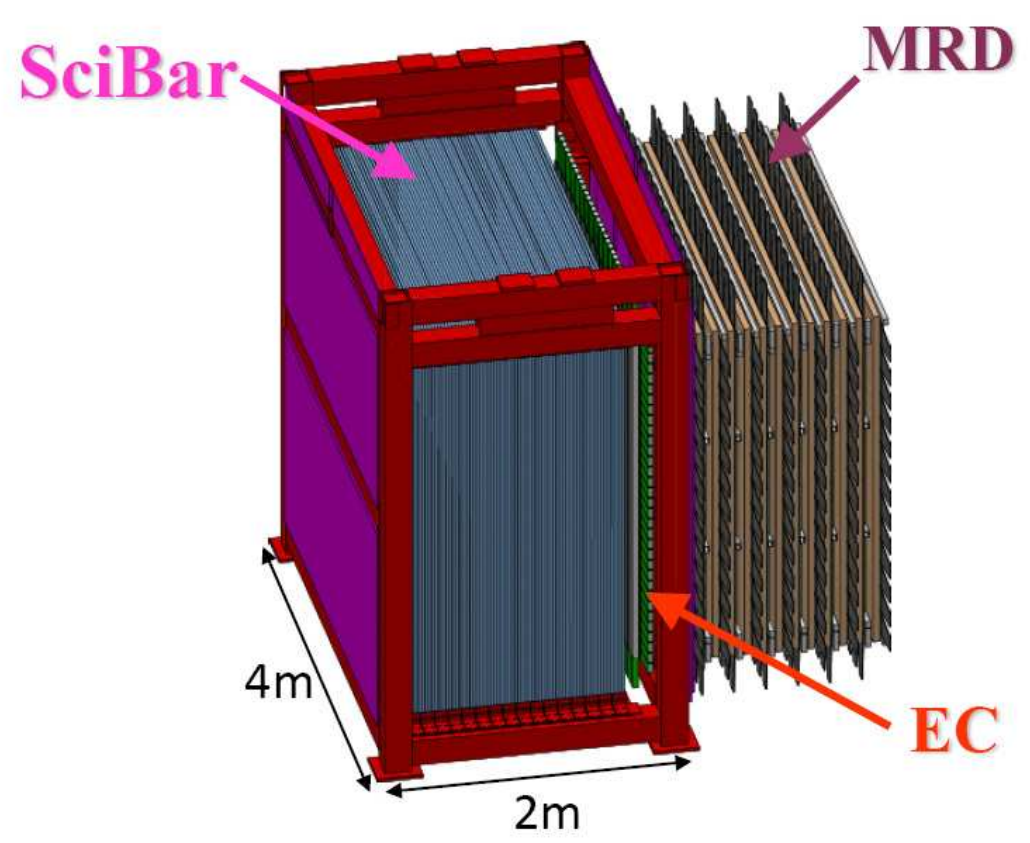

Figure 3.5: Schematic view of the entire SciBooNE detector.

scintillator tracker, it can distinguish charged particles (e.g. muons from protons) based on their energy deposition per unit length $(d E / d x)$.

The 15 ton SciBar sub-detector 43] (10.6 ton fiducial volume for antineutrino events to be accepted) consists of $143361.3 \mathrm{~cm} \times 2.5 \mathrm{~cm} \times 300 \mathrm{~cm}$ rectangular extruded polystyrene $\left(\mathrm{C}_{8} \mathrm{H}_{8}\right)$ scintillator (1\% PPO and $0.03 \%$ POPOP by weight) strips glued together in alternating layers of vertically and horizontally arranged planes to construct a $3 \times 3 \times 1.7 \mathrm{~m}^{3}$ volume. Each scintillator strip is coated with a co-extruded reflector material $(0.25 \mathrm{~mm}$ thick, $15 \% \mathrm{TiO}_{2}$ infused polystyrene) and has a $1.8 \mathrm{~mm}$ diameter circular hole which houses a single $1.5 \mathrm{~mm}$ wavelength shifting fiber (WLS) so that light created from passing charged particles in the scintillator is collected and wavelength shifted by the WLS fiber as shown in Figure 3.6. A plane is 116 strips glued together with 124 planes in total. Figure 3.7 shows a three-dimensional view of the scintillator strips of SciBar.

The ionization in the scintillator strips in SciBar follow Birk's law, a relationship between the visible ionization energy loss $\Delta E_{v i s}$ and the actual deposited energy $\Delta E$, which is:

$$
\frac{\Delta E_{v i s}}{\Delta E} \propto \frac{1}{1+c \times d E /\left.d x\right|_{\exp }}
$$




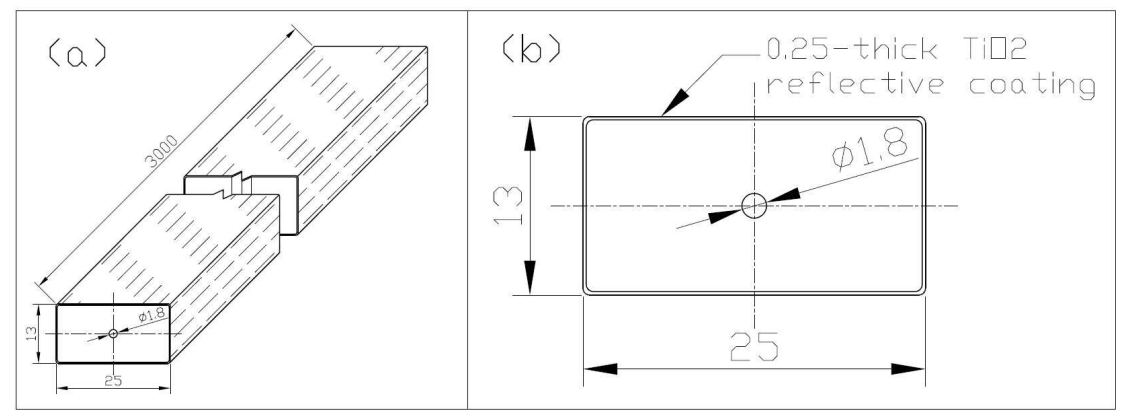

Figure 3.6: Schematic drawing of a scintillator strip in three-dimensional view (a) and cross sectional view (b). All units are in $\mathrm{mm}$.

where $d E /\left.d x\right|_{\text {exp }}$ is the expected energy deposition per unit length and $c$ is Birk's constant which depends on the material. The Birks constant for the SciBar scintillator strip is measured to be $0.0208 \pm 0.0023 \mathrm{~cm} / \mathrm{MeV}$ [4], using a prototype of SciBar in a proton beam.

For the readout of the scintillator strips, each WLS fiber is attached to a channel on a 64channel multi-anode photomultiplier tube, or MA-PMT, (MA-PMT, Hamamatsu H8804) in groups of 64 WLS fibers as shown in Figure 3.8. The WLS fibers are brought out together on one side of the detector and are held in place by a plastic alignment "cookie" which interfaces directly to a 64-channel MA-PMT. The average gain for the 64 channels is $6 \times 10^{5}$. The crosstalk (light from one fiber channel affecting a nearby fiber channel on the PMT) was measured to be $3.15 \% \pm 0.4 \%$ for an adjacent channel [41]. Charge and timing information is then read out by a front end board (FEB) attached to the MA-PMT. Data acquisition boards (DAQ) read out $8 \mathrm{FEBs}$ with a typical pedestal width less than 0.3 photo electrons (pe).

The readout is built with a special calibration system as shown in Figure 3.8. A single clear fiber is placed in the center of each group of 64 WLS fibers which injects light from an blue LED matched to the WLS fiber's absorption spectrum. The response to the calibration LED light, which is triggered by an external NIM module, provides a measurement of the gain of the MA-PMTs over time as well as indicating which channels are dead. The relative gain of the MA-PMTs is monitored every 8 hours with a precision of $0.1 \%$ and corrected 


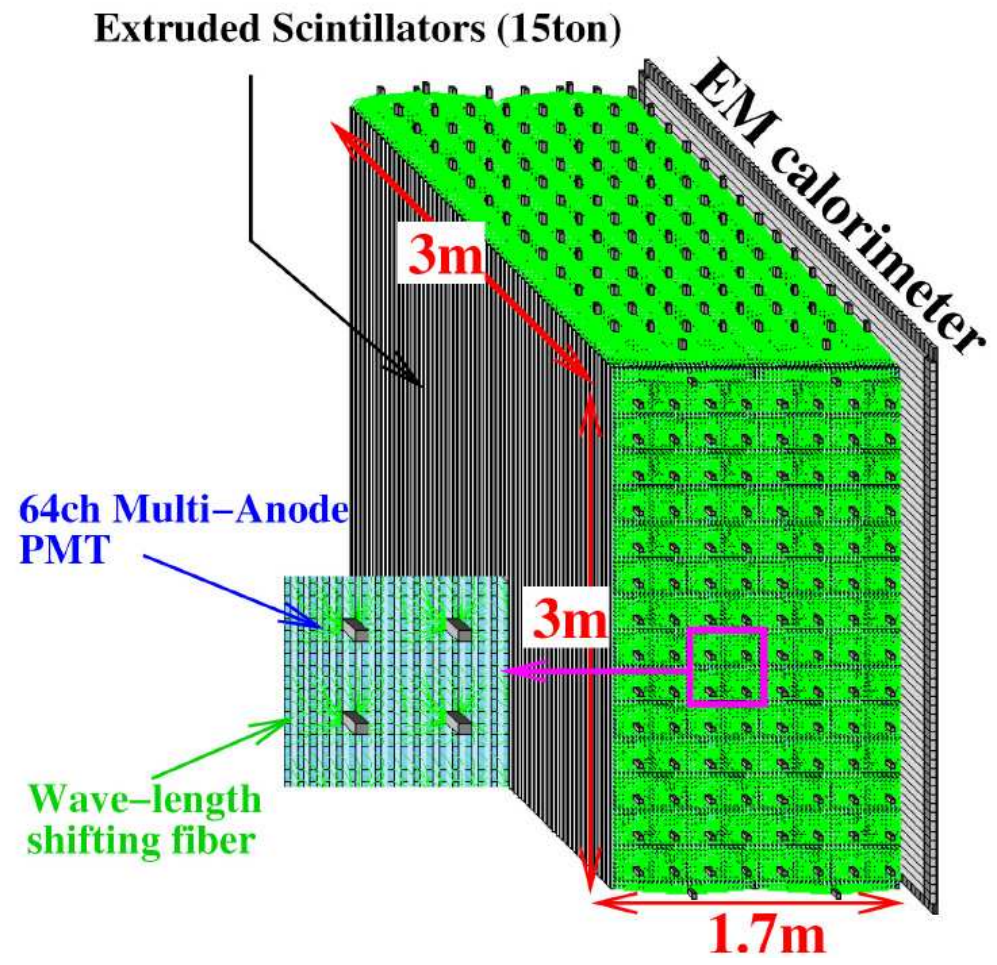

Figure 3.7: Three-dimensional view of the SciBar and EC sub-detectors. The SciBar subdetector consists of alternating scintillator strips (shown in white), each instrumented with a green wavelength shifting fiber down its the center (shown in the green lateral exterior of SciBar), and read out by MA-PMTs (grey rectangle shown in the inset). The EC subdetector consists of two planes of scintillating fibers covered with lead foil. The antineutrino beam is assumed to be incident from the left.

for the gain drift for the MA-PMTs. Over the course of the entire SciBooNE run, the gain was stable to $\pm 2 \%$ and only 4 channels out of 14,336 failed permanently.

The energy scale of each channel (each scintillator strip) is calibrated using cosmic muons. More details on the scintillator, WLS fiber, readout electronics, gain monitoring system, and energy calibration can be found in Reference 41].

The SciBar sub-detector also acts as the primary veto for charged particles coming into the detector from the outside. The charged particles coming in from outside the detector will deposit energy in the scintillator strips located in most upstream layers and on the 


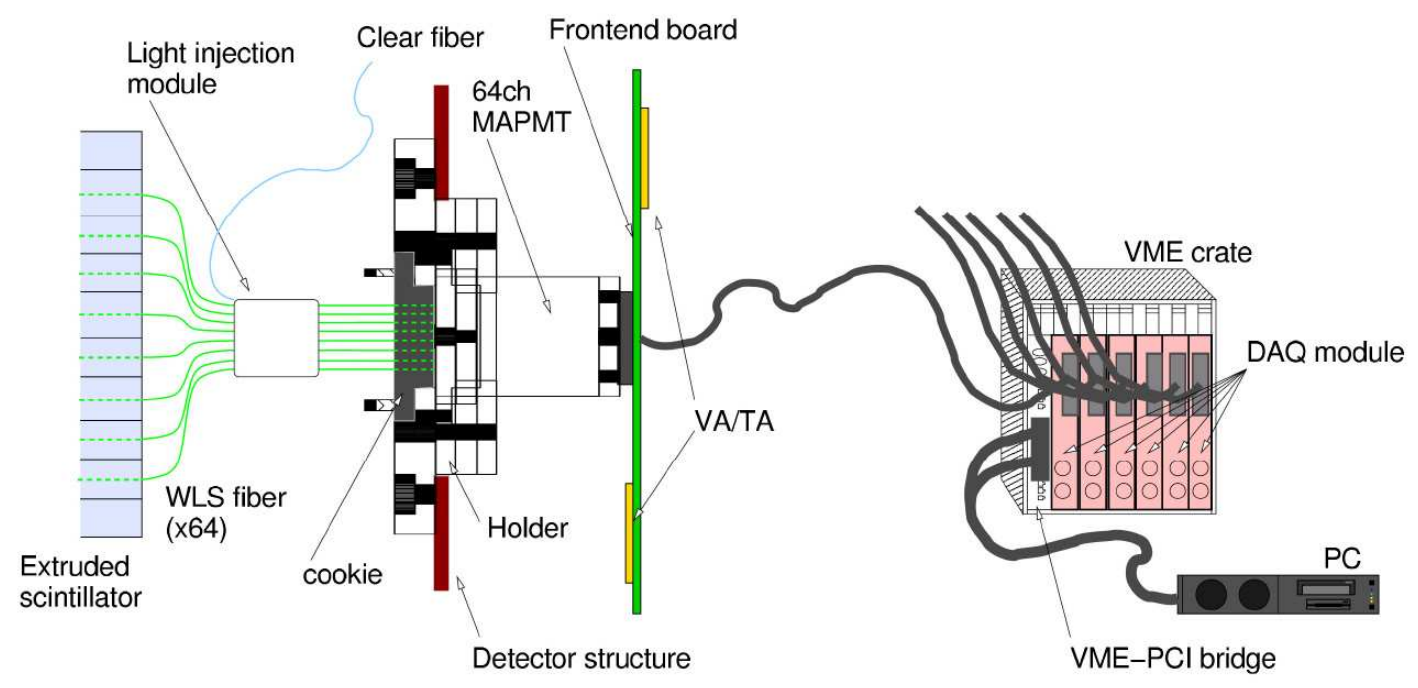

Figure 3.8: Schematic diagram of the SciBar readout.

top and sides of SciBar. The presence of these energy depositions used to reject charged particles coming in from the outside during the event selection process.

SciBar was originally designed and built as a near detector for the K2K experiment 43 . After K2K was completed, the SciBar detector was disassembled, shipped to FNAL, and then re-built there for SciBooNE.

\subsubsection{The EC Sub-detector}

The EC sub-detector is an electromagnetic calorimeter, installed immediately downstream of the SciBar sub-detector, and is designed to identify photons from $\pi_{0}$ decays and measure the $\nu_{e}$ contribution in the neutrino and antineutrino beams by detecting electron showers. A three-dimensional view is shown in Figure 3.7. The EC sub-detector is a two plane (vertical and horizontal) "spaghetti"-type calorimeter. Each plane consists of 32 modules, which are $1 \mathrm{~mm}$ scintillating fibers embedded in lead foil with dimensions of $262 \times 8.4 \times 4.2 \mathrm{~cm}^{3}$, arranged side by side. Both EC planes collectively cover an active area of $2.7 \times 2.6 \mathrm{~m}^{2}$. The scintillating fibers are bundled and read out at both ends by PMTs. The EC sub-detector was originally built for the CHORUS experiment [45], later used in conjunction with SciBar for the K2K experiment, before finally being used in the SciBooNE experiment. Since 
neither events with $\pi_{0} \mathrm{~s}$ and $\nu_{e}$ events are considered in this analysis, data taken from the EC sub-detector is not used in the analysis though it is taken into account in the SciBooNE detector geometry for data and MC.

\subsubsection{The MRD Sub-detector}

The MRD sub-detector is installed downstream of the EC sub-detector and designed to measure $\mu^{ \pm}$momentum up to $1.2 \mathrm{GeV} / \mathrm{c}$ for the $\mu^{ \pm}$that are created from neutrino and antineutrino interactions in SciBar. The MRD consists of 12 iron plates, each $5 \mathrm{~cm}$ thick, sandwiched between 13 alternating horizontal and vertical scintillator planes as shown in Figure 3.9. The average density of the iron plates is $7.841 \pm 0.002 \mathrm{~g} / \mathrm{cm}^{3}$ and the thickness of each iron plate was measured to $1 \%$ accuracy prior to construction of the MRD. Each scintillator plane is made of $20 \mathrm{~cm}$ wide and $6 \mathrm{~mm}$ thick scintillator paddles. Each vertical scintillator plane ( 7 total) consists of $138 \mathrm{~cm}$ long paddles arranged in a $2 \times 15$ array for an active area of $276 \times 300 \mathrm{~cm}^{2}$ while each horizontal scintillator plane (6 total) consists of 155 cm long paddles arranged in a $13 \times 2$ array for an active area of $260 \times 310 \mathrm{~cm}^{2}$. The 362 scintillator paddles in the MRD are read out via 362 individual 2 inch PMTs. The MRD was constructed for SciBooNE at FNAL, primarily by using parts recycled from past FNAL experiments.

\subsubsection{Events in SciBooNE}

The different neutrino and antineutrino interactions in SciBooNE create different discrete tracks and hits. By identifying the signature of the tracks and hits in all three sub-detectors in conjunction, the specific neutrino or antineutrino interaction can be inferred. Figure 3.10 shows the different signatures of tracks and hits and its associated antineutrino interaction. Muon antineutrino interactions can produce a long track that extends from SciBar to MRD. Electron antineutrino interactions produce a track in SciBar that ends with energy deposition in the EC. Antineutrino interactions producing $\pi^{0}$ creates two tracks in SciBar that point to the same vertex. 


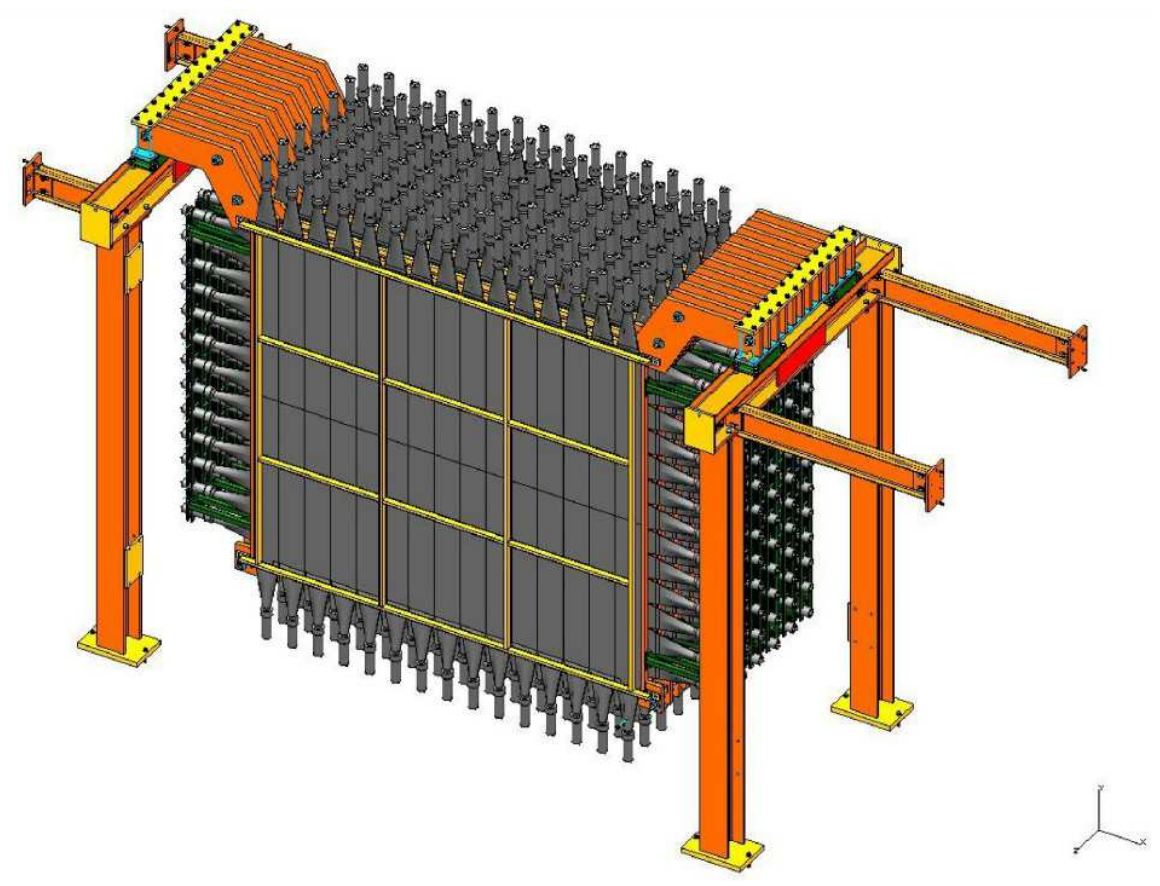

Figure 3.9: Three-dimensional view of the MRD sub-detector.

\subsection{The MiniBooNE Detector}

MiniBooNE consists of a spherical $12.2 \mathrm{~m}$ diameter tank containing 800 tons of mineral oil beneath at least $3 \mathrm{~m}$ of earth overburden, as shown in Figure 3.11. The detector is divided into an inner, active region to detect the antineutrinos from the beam and an outer, veto region to reject charged particles coming from outside the detector. Within the inner, active region is the fiducial volume of the detector, a sphere $10 \mathrm{~m}$ in diameter with a mass of 450 tons. A $2 \mathrm{GeV}$ muon roughly travels the full $12 \mathrm{~m}$ length of the tank. Figure 3.12 shows a schematic view of the MiniBooNE detector.

The mineral oil, Marcol $7\left(\mathrm{CH}_{2}\right)$, serves as both the target for the antineutrino beam and the light producing medium. The mineral oil has many favorable properties. The mineral oil has a density of $0.845 \pm 0.001 \mathrm{~g} / \mathrm{cm}^{3}$ and a refractive index of 1.47 . The refractive index is higher than water (1.33), allowing a higher Cherenkov light fraction for charged particles and a lower Cherenkov threshold than water. In addition to Cherenkov light, the mineral oil also scintillates light as charged particles pass through it. Four different fluorophores 


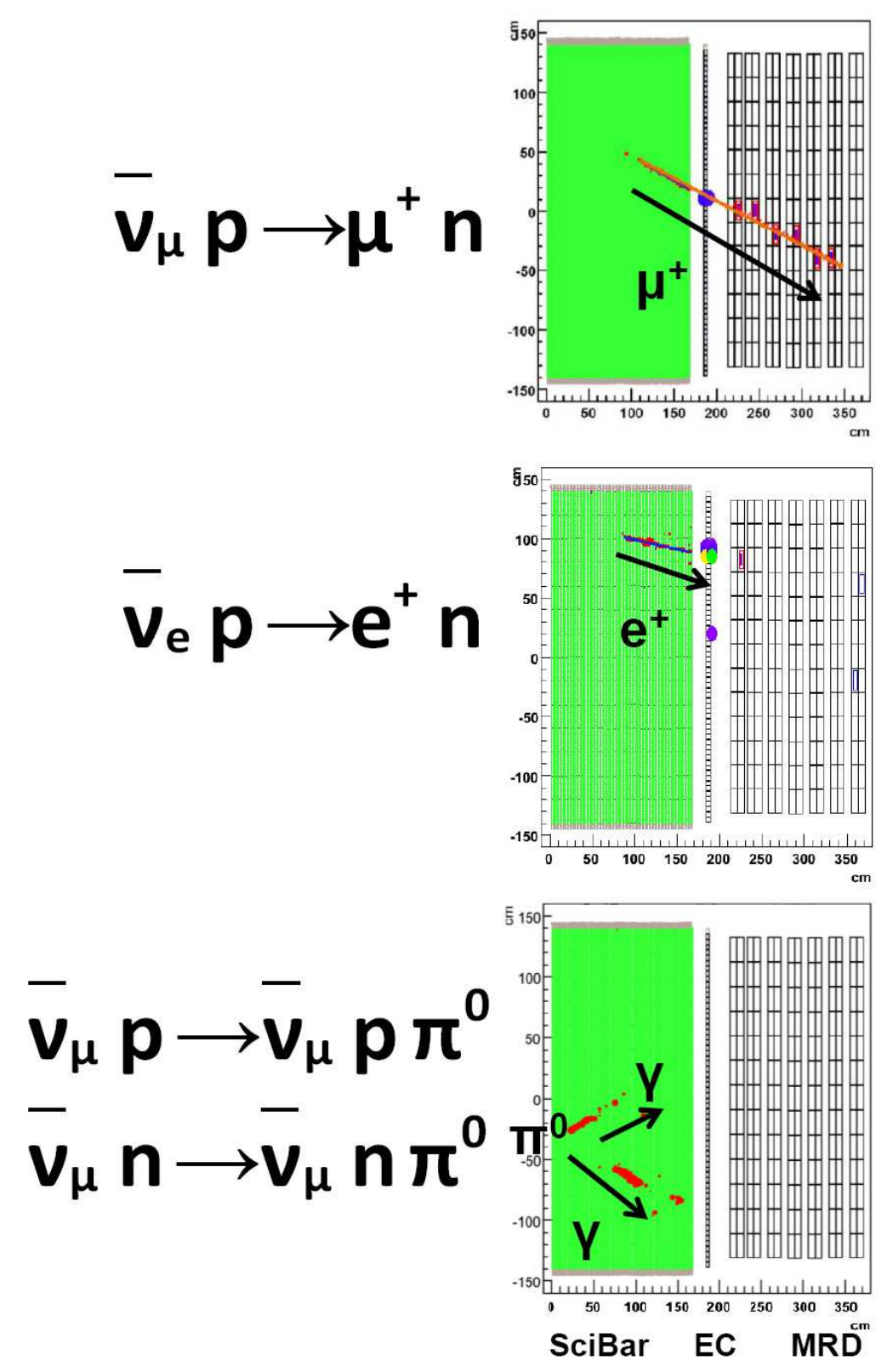

Figure 3.10: Types of antineutrino interactions in SciBooNE and their associated detection signatures.

were detected in studies of the time fluorescence of the mineral oil. The Cherenkov and scintillation light Raleigh and Raman scatters through the mineral oil with an absorption length of about $18 \mathrm{~m}$. 


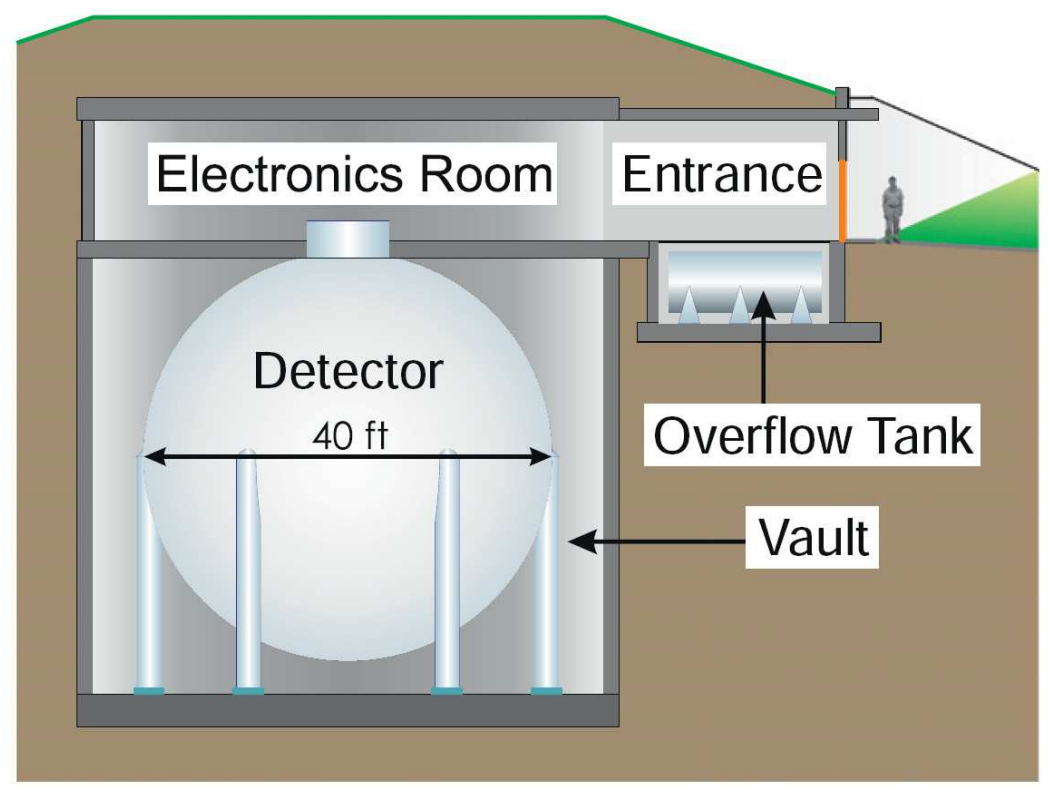

Figure 3.11: Schematic view of the MiniBooNE enclosure.

The detector is instrumented with 12808 inch photomultiplier tubes (PMTs) in the inner, active region. The PMTs detect Cherenkov and scintillation light coming from passing charged particles created from neutrino and antineutrino interactions in the fiducial volume. The PMTs in the inner region face radially inward towards the spherical fiducial volume. The PMTs are arranged in horizontal layers on opaque panels with latitudinal hoops to allow the mineral oil to flow between the inner, active region and the outer, veto region without light leakage. The inner region is covered or painted black to reduce re-scattering of light.

The detector is instrumented with 2408 inch PMTs in the outer, veto region. The PMTs in the outer region detect Cherenkov light from charged particles coming from outside detector. The PMTs in the outer region are mounted back to back and face either upwards or downwards. The outer region is painted white to increase light re-scattering.

The PMTs are operated with a gain of approximately $1.6 \times 10^{7}$ at $+2000 \mathrm{~V}$. Charge and timing information are read out when the charge on a PMT is greater than $2 \mathrm{mV}$ (about 0.1 photoelectron). Each PMT readout is a PMT "hit". The PMTs have a dead time, the minimum time between successive PMT hits, of roughly 200-300 ns depending on the time 


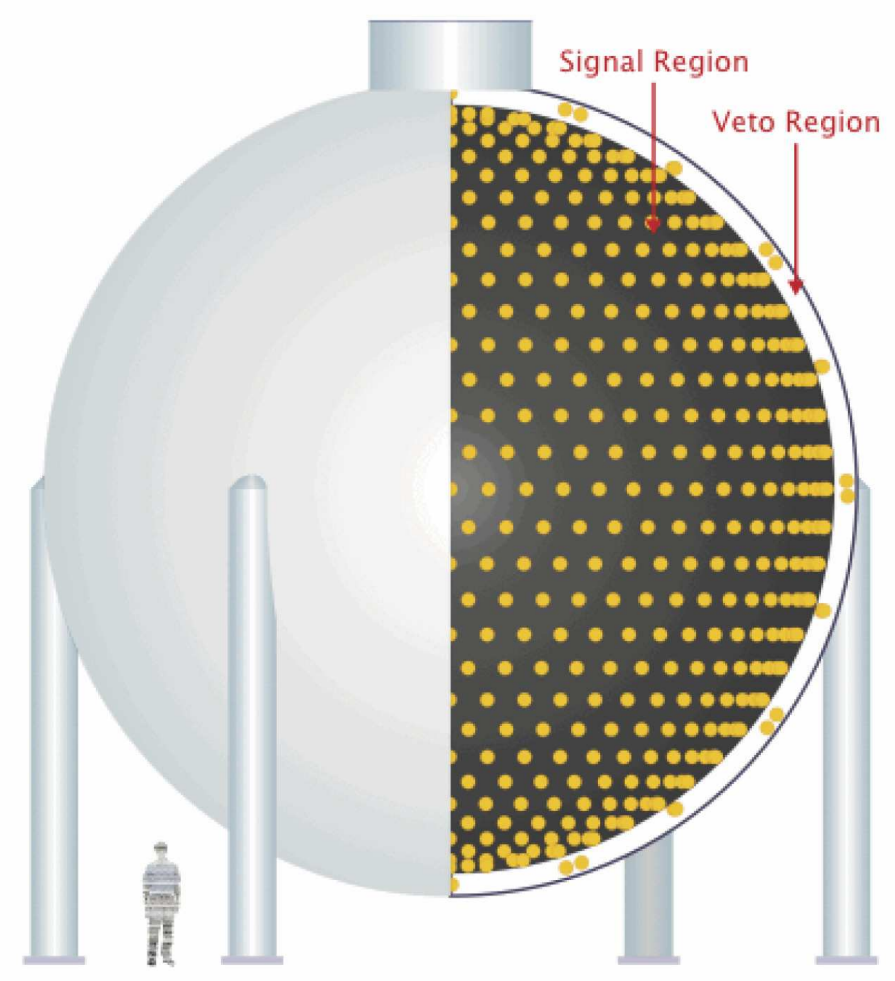

Figure 3.12: Schematic view of the MiniBooNE detector.

of the first hit. A photo of a MiniBooNE PMT is shown in Figure 3.13 .

The MiniBooNE data acquisition system (DAQ) digitizes the analog signal from the PMTs, which includes time and charge information, and stores the information for 200 $\mu \mathrm{s}$. The information is processed and read out if one of the physics triggers is set to be true. The relevant physics triggers for this analysis are the beam and strobe triggers. The beam trigger comes from the accelerator clock and does not depend on PMT activity in the detector. The beam trigger opens a $19.2 \mu$ s beam readout window starting from $5 \mu$ s before the $1.6 \mu$ s beam spill and holds off acceptance of any subsequent triggers for the full length of the $19.2 \mu$ s beam readout window. The strobe trigger is a $2.01 \mathrm{~Hz}$ pulser that provides an unbiased sample of beam-off events, which are mainly cosmic muon events.

A variety of methods are used to calibrate the MiniBooNE detector. Due to the small $3 \mathrm{~m}$ overburden, cosmic muons enter the MiniBooNE detector at a rate of $10 \mathrm{kHz}$. All these cosmic muons can be vetoed by the PMTs in the outer region but they are also 


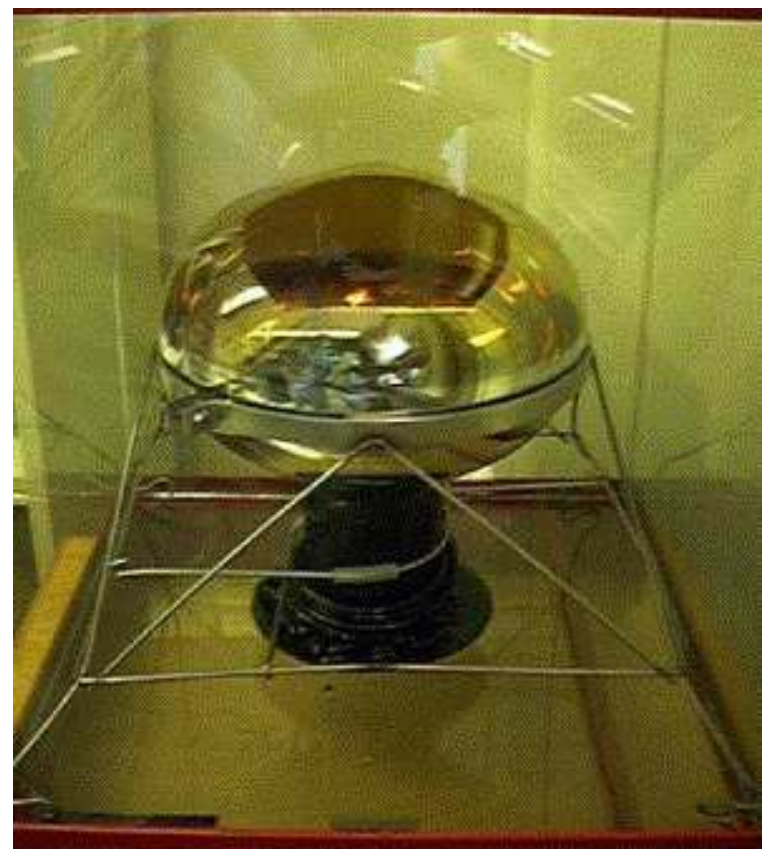

Figure 3.13: Photo of a MiniBooNE PMT.

used as a good calibration source for the detector. A laser calibration system is built to also calibrate the detector. In addition, further calibration can be done by reconstructing known quantities such as the $\pi^{0}$ mass and Michel electron energy distribution.

Additional details about the MiniBooNE detector can be found under Reference 42 .

\subsubsection{Events in MiniBooNE}

The different neutrino and antineutrino interactions in MiniBooNE create different patterns of PMT hits in the inner, active region. By distinguishing and identifying the pattern of PMT hits, the specific neutrino or antineutrino interaction can be inferred. Figure 3.14 shows the different types of PMT patterns and its associated antineutrino interaction. Muon antineutrino interactions produce a sharp, distinct ring or circle of PMT hits. Electron antineutrino interactions produce a blurred ring of PMT hits. Antineutrino interactions producing $\pi^{0}$ creates two intersecting blurred rings of PMT hits. 


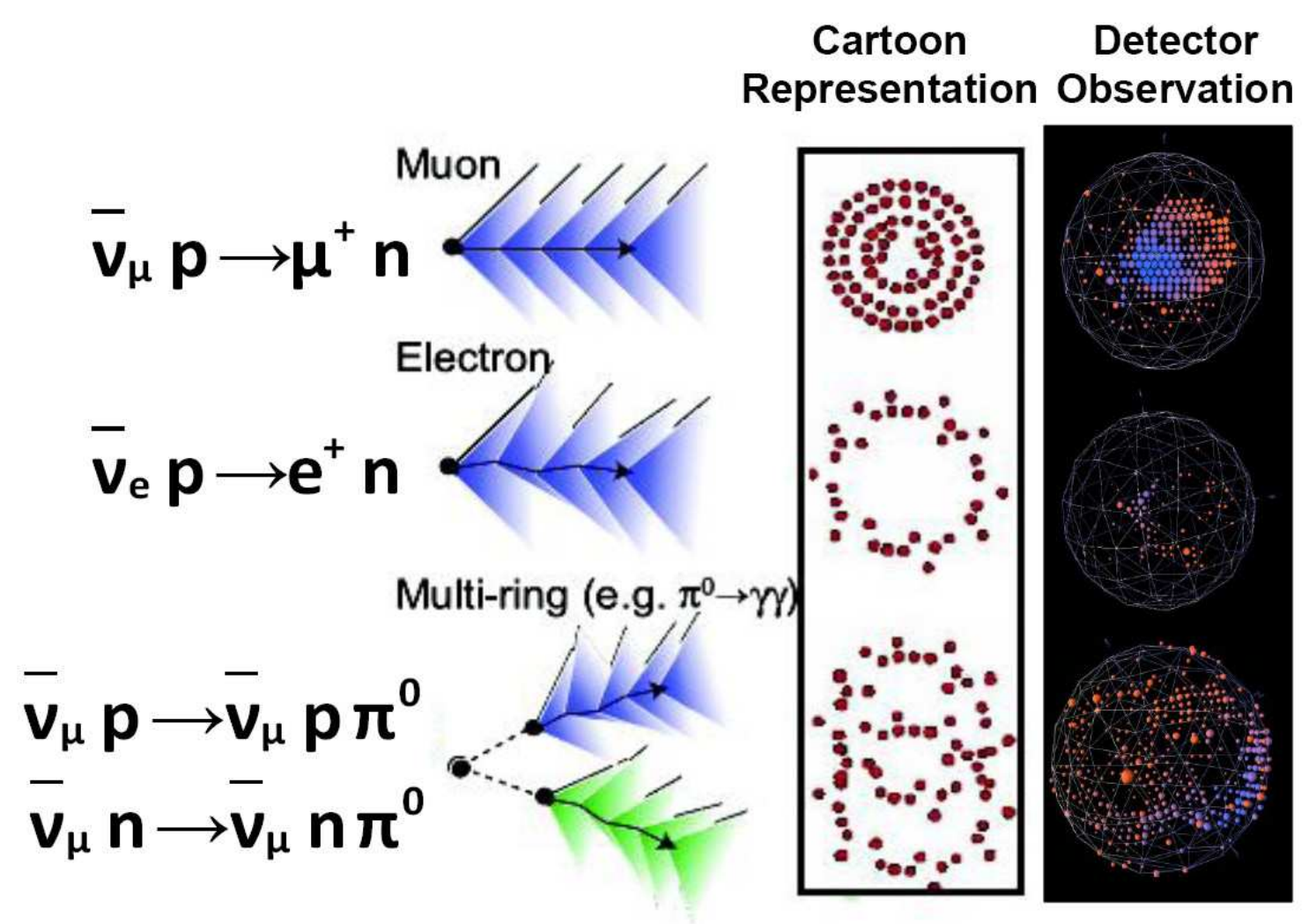

Figure 3.14: Types of antineutrino interactions in MiniBooNE and their associated detection signatures. 


\section{Chapter 4}

\section{Monte Carlo Simulation}

The purpose of this chapter is to describe the Monte Carlo (MC) simulation. The MC simulation is used to estimate the expected signal of the analysis, the uncertainties and sensitivity of the analysis, and the background contamination.

The chapter is organized in order of antineutrino progression sequence from production, to propagation, to interaction, and finally detection. The simulation of the antineutrino beam from the BNB is first detailed. Then, the method for the propagation of the predicted antineutrino flux to both detectors is explained. The modeling of the antineutrino beam's neutrino and antineutrino interactions with the detectors' target nuclei are described next. Finally, the modeling of the interactions from the resulting particles created in the initial neutrino and antineutrino interactions as they pass through the detector materials leading to the electronic signals obtained are covered.

\subsection{Monte Carlo Events and Event Weighting}

In order for the information from the MC simulation to be complete and versatile, at each step of the MC simulation, all the information related to an event including the particle properties, its interactions, and its history must be passed on event by event to the next step so that the information can be traced back and used for further analysis if necessary. Therefore, the complete MC simulation is composed of many events, where each MC event contains all the particle information, the interaction information, and the history informa- 
tion that relates to the event.

The composition of the MC simulation from MC events possesses additional advantages. Statistical uncertainties in the MC simulation can be minimized using a technique called "event weighting". In event weighting, many more MC events are generated than the number of events expected in data. Each MC event is then given a "weight", which is based on the probability of that event occurring. When the $\mathrm{MC}$ events are combined to form distributions, the $\mathrm{MC}$ events are combined in accordance to the size of their weights so that the MC distribution has the same predicted normalization as the data distribution. But since the MC distribution was created from many more MC events than data events, the statistical uncertainties in the MC distribution are much less than the corresponding statistical uncertainties in the data distribution and therefore, the MC statistical uncertainties can be effectively ignored. For both SciBooNE and MiniBooNE MC events, roughly an order of magnitude more events are generated in MC than expected in data to minimize MC statistical uncertainties. In addition, event weighting can be further used to modify the relative proportion of certain selected $\mathrm{MC}$ events relative to all other $\mathrm{MC}$ events by adjusting their corresponding weight values.

\subsection{Booster Neutrino Beamline Antineutrino Flux}

The BNB antineutrino flux is the first step in the MC simulation and serves both the MiniBooNE and SciBooNE MC event simulations further down the simulation process. The simulation of the antineutrino beam consists of two main sections: simulation of the meson production from the proton-Beryllium collision and simulation of the meson focusing, propagation, and decay into the antineutrino beam. The simulation of the meson production can further be divided into the modeling of the proton beam and Beryllium target, modeling of the proton-Beryllium elastic and inelastic scattering interactions, and the simulation of the $\pi^{ \pm}, K^{ \pm}$, and $K^{0}$ meson production. 


\subsubsection{Proton Beam, Beryllium Target, Beamline Geometry, and Proton- Beryllium Interaction Modeling}

The proton beam, Beryllium target, and surrounding beamline geometry are first modeled as accurately as possible. Using information from the beamline monitors, the incident proton beam is simulated to be centered on the Beryllium target, with a Gaussian spread in position of $\sigma_{x}=1.51 \mathrm{~mm}$ and $\sigma_{y}=0.73 \mathrm{~mm}$ and an angular spread of $\sigma_{\theta_{x}}=0.66 \mathrm{mrad}$ and $\sigma_{\theta_{y}}=0.40 \mathrm{mrad}$. Variations in the incident proton beam (changes in the focus point of the beam, size of the transverse and angular spread of the beam, etc) have tiny effects of less than $1 \%$ on the resulting antineutrino flux. The uncertainties in the resulting antineutrino beam from the accuracy of the Beryllium target and beamline geometry modeling are so small (significantly less than 1\%) that the Beryllium target and beamline geometry modeling uncertainties are ignored.

The majority of protons in the proton beam interact in the Beryllium target. The proton interactions in surrounding beamline material are modeled using the default GEANT4 hadronic models. The total interaction cross section of primary proton interactions on Beryllium is the sum of the elastic and the inelastic cross section. Inelastic collisions can

be either quasielastic or reaction interactions where mesons such as $\pi^{ \pm}, K^{ \pm}$, and $K^{0}$ can be produced.

The Glauber model is used to simulate the total cross section of proton on Beryllium. The Glauber model is compared with existing cross section data as shown in Figure 4.1. More information on the Glauber model can be found in Reference [3]. The total interaction cross section is $\sigma_{\text {total }}=285 \pm 15 \mathrm{mb}$ for protons at $8.9 \mathrm{GeV} / \mathrm{c}$. The inelastic cross section is parameterized based on Figure 4.1 for use in the MC simulation. The elastic cross section is calculated by subtracting $\sigma_{\text {inelastic }}$ from $\sigma_{\text {total }}$. The quasielastic cross section is theoretically calculated and given a larger $\pm 20 \mathrm{mb}$ uncertainty. The proton-Beryllium cross section uncertainties result in a few percent uncertainty in the antineutrino flux.

\subsection{2 $\pi$ and $K$ Meson Production Simulation}

The $\pi^{+}, \pi^{-}, K^{+}, K^{-}$, and $K^{0}$ production cross sections from $8.9 \mathrm{GeV} / \mathrm{c}$ proton-Beryllium interactions are simulated separately, each based on a different custom model and/or parametriza- 

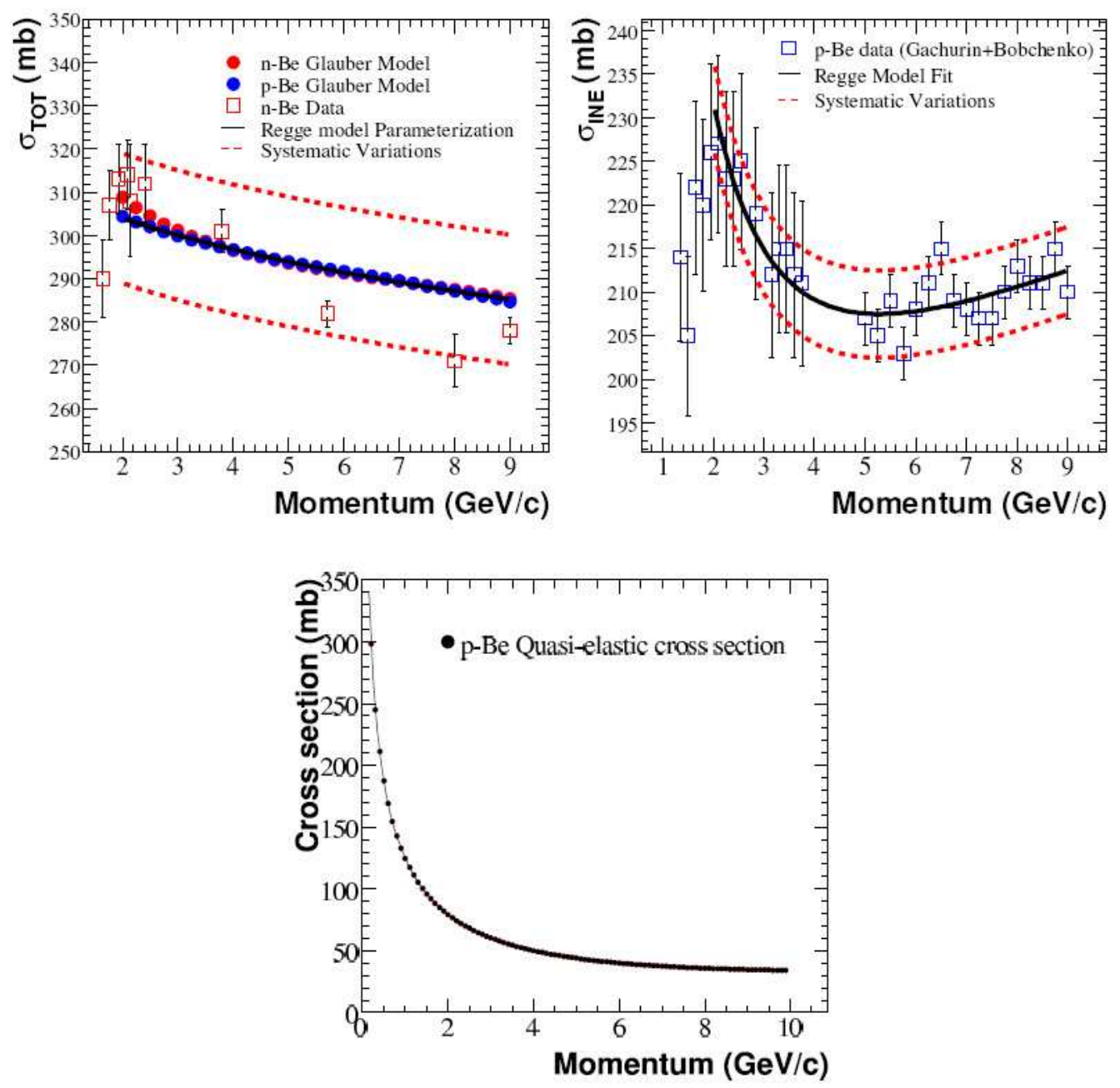

Figure 4.1: The cross section measurements of proton/Beryllium interactions with the Glauber model. The top left figure shows the total hadronic cross section for Beryllium in neutron/Beryllium interactions data (box), neutron/Beryllium interactions prediction (red circle), and proton/Beryllium interactions prediction (blue circle) within the allowed systematic uncertainties (red dash). The top right figure shows the total inelastic hadronic cross section for Beryllium in proton/Beryllium data (blue box) and parametrization (black line) within systematic uncertainties (red dash). The bottom middle figure shows the total quasielastic hadronic cross section for proton/Beryllium interactions with parametrization (black line). 
tion. These mesons are parent particles for the antineutrinos and background neutrinos in the antineutrino beam.

For $\pi^{+}$and $\pi^{-}$production, the simulation uses the Sanford-Wang (SW) parametrization:

$$
\frac{d^{2} \sigma}{d p d \Omega}(p, \theta)=c_{1} p^{c_{2}}\left(1-\frac{p}{p_{B}-c_{9}}\right) \exp \left(-c_{3} \frac{p^{c_{4}}}{p_{B}^{c_{5}}}-c_{6} \theta\left(p-c_{7} p_{B} \cos ^{c_{8}} \theta\right)\right)
$$

where $\frac{d^{2} \sigma}{d p d \Omega}$ is the double differential cross section, $p$ is the total momentum of the meson in $\mathrm{GeV} / \mathrm{c}, \theta$ is the angle of the meson with respect to the incident proton in radians, $p_{B}$ is the momentum of the incident proton in $\mathrm{GeV} / \mathrm{c}$, and $c_{1}, . ., c_{9}$ are parameters to be determined in the fit to the production data. For $\pi^{+}$and $\pi^{-}$production, the $c_{1}, . ., c_{9}$ parameters from production data are obtained by fitting the HARP 46, and BNL E910 47 data and shown in Table 4.1.

Table 4.1: SW fit parameters for $\pi^{+}$and $\pi^{-}$production from HARP and BNL E910 data.

\begin{tabular}{c|c|c|c|c|c|c|c|c|c}
\hline \hline & $\mathrm{c} 1$ & $\mathrm{c} 2$ & $\mathrm{c} 3$ & $\mathrm{c} 4$ & $\mathrm{c} 5$ & $\mathrm{c} 6$ & $\mathrm{c} 7$ & $\mathrm{c} 8$ & $\mathrm{c} 9$ \\
\hline$\pi^{+}$ & 220.7 & 1.080 & 1.000 & 1.978 & 1.32 & 5.572 & 0.0868 & 9.686 & 1 \\
$\pi^{-}$ & 213.7 & 0.9379 & 5.454 & 1.210 & 1.284 & 4.781 & 0.07338 & 8.329 & 1 \\
\hline \hline
\end{tabular}

The uncertainties for both the $\pi^{+}$and $\pi^{-}$production simulation are determined by 1000 spline fits to their respective $\pi^{+}$and $\pi^{-}$HARP and BNL E910 data and are centered around their respective SW parametrization. Figure 4.2 shows the SW parametrization with the spline fit range for $\pi^{-}$and Figure 4.3 shows the SW parametrization with the spline fit range for $\pi^{+}$. Notice that the spline curves nicely matches the HARP error bars, except below $1 \mathrm{GeV} / \mathrm{c}$. Extremely few neutrinos and antineutrinos detected by MiniBooNE and SciBooNE come from $\pi^{+}$and $\pi^{-}$with momenta below $1 \mathrm{GeV} / \mathrm{c}$.

For $K^{+}$production, the Feynman scaling hypothesis is used to parameterize the $\mathrm{K}^{+}$ double differential cross section. Feynman scaling relates $K^{+}$production measurements at different proton beam energies to the expected production at the BNB proton beam energy. The Feynman scaling variable $x_{F}$ defined as $p_{\|}^{c m} / p_{\|}^{\text {max,cm }}$ where $p_{\|}^{c m}$ and $p_{\|}^{\text {max,cm }}$ are the parallel component of the momentum of the produced particle in the center-of-mass frame and the maximum value of $p_{\|}^{c m}$ for the given reaction, respectively, is used in the 

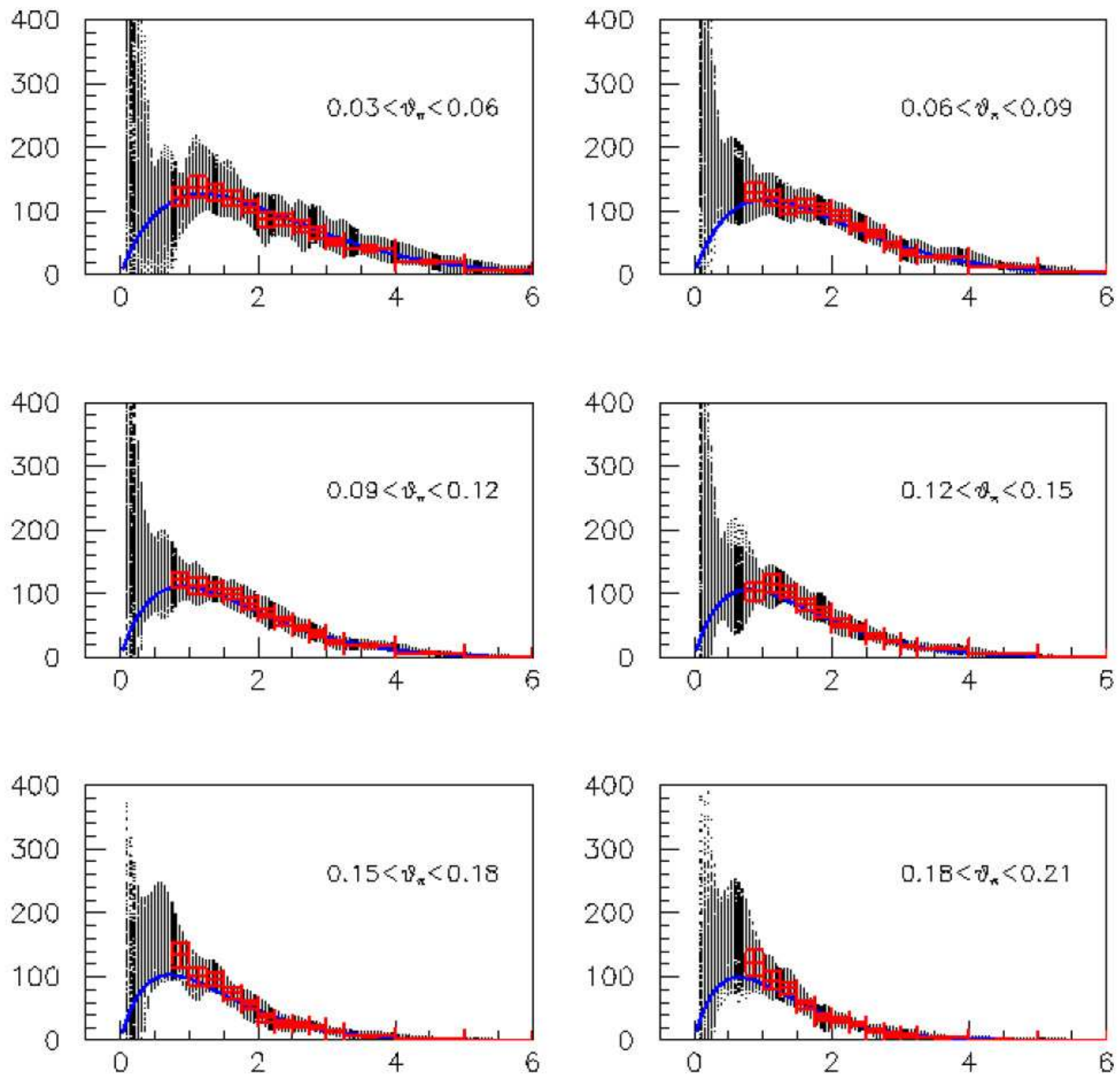

Figure 4.2: $\pi^{-}$production cross section HARP data at $8.9 \mathrm{GeV} / \mathrm{c}$ from proton-Beryllium collisions (red crosses) as a function of $\pi^{-}$momentum $\left(p_{\pi^{-}}\right)$in $\mathrm{GeV} / \mathrm{c}$ for different intervals of $\pi^{-}$angle $\left(\theta_{\pi^{-}}\right)$. The best fit SW parametrization (blue line) and 40 out of the 1000 spline fits to the HARP data (black lines) are also shown.

parametrization. The double differential cross section using $x_{F}$ is expressed as:

$$
\frac{d^{2} \sigma}{d p d \Omega}=\frac{p^{2}}{E^{2}} \times c_{1}\left(1-\left|x_{F}\right|\right) \exp \left(-c_{2} p_{T}-c_{3}\left|x_{F}\right|^{c_{4}}-c_{5} p_{T}^{2}-c_{7}\left|p_{T} \times x_{F}\right|^{c_{6}}\right)
$$



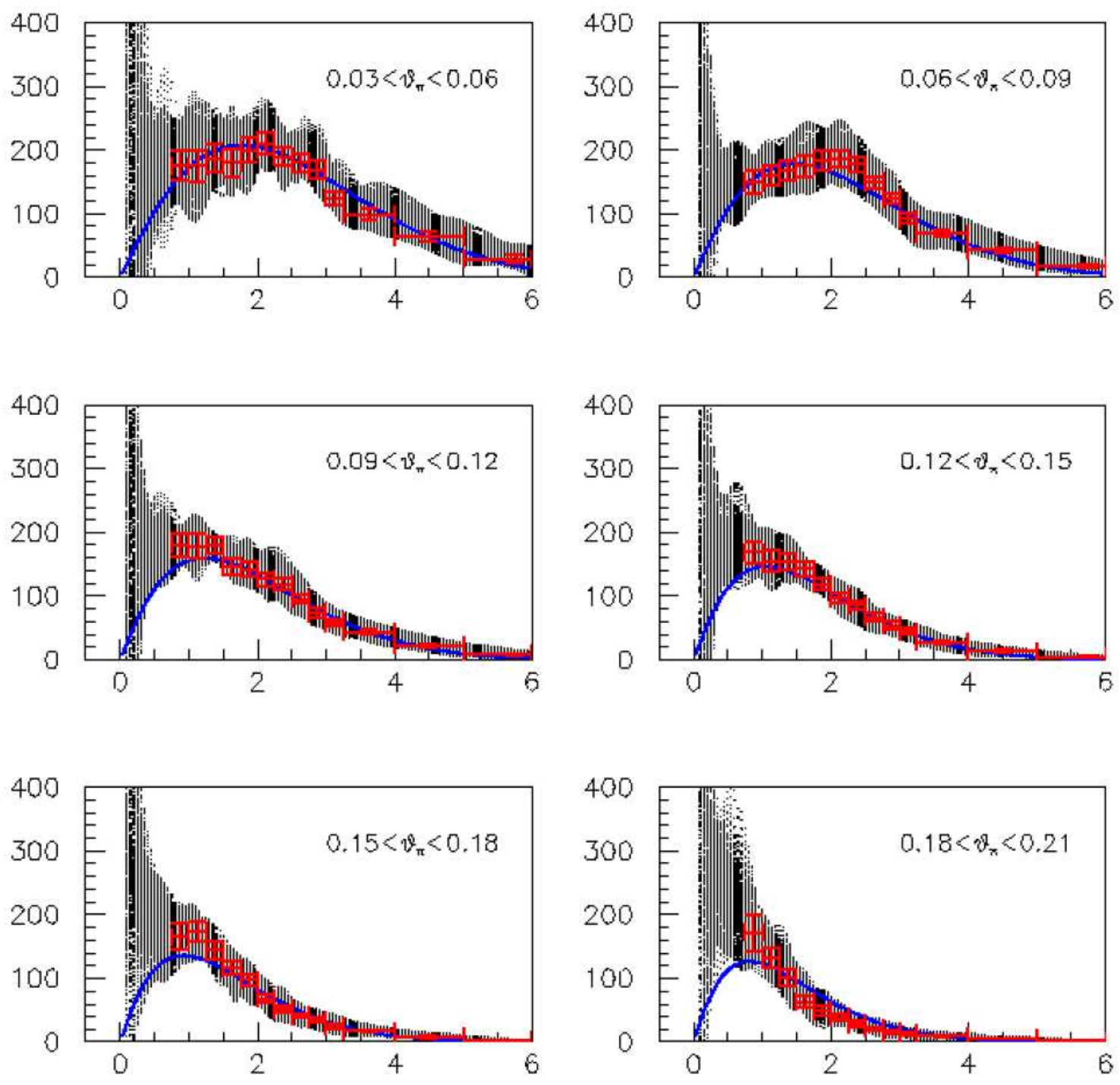

Figure 4.3: $\pi^{+}$production cross section HARP data at $8.9 \mathrm{GeV} / \mathrm{c}$ from proton-Beryllium collisions (red crosses) as a function of $\pi^{+}$momentum $\left(p_{\pi^{+}}\right)$in $\mathrm{GeV} / \mathrm{c}$ for different intervals of $\pi^{+}$angle $\left(\theta_{\pi^{+}}\right)$. The best fit SW parametrization (blue line) and 40 out of the 1000 spline fits to the HARP data (black crosses) are also shown.

where $p, p_{T}$, and $E$ are the momentum, the transverse component of the momentum, and energy of produced $K^{+}$, respectively. $c_{1}, . ., c_{7}$ are parameters from fitting to production data at different proton beam energies and are listed in Table 4.2 48. The uncertainties in 
the simulation of the $K^{+}$come from variations in the Feynman scaling parameters $c_{1}, . ., c_{7}$ in the fit.

Table 4.2: Feynman scaling fit parameters for $K^{+}$production.

\begin{tabular}{c|c|c|c|c|c|c|c}
\hline \hline & $\mathrm{c} 1$ & $\mathrm{c} 2$ & $\mathrm{c} 3$ & $\mathrm{c} 4$ & $\mathrm{c} 5$ & $\mathrm{c} 6$ & $\mathrm{c} 7$ \\
\hline$K^{+}$ & 11.29 & 0.87 & 4.75 & 1.51 & 2.21 & 2.17 & 1.51 \\
\hline \hline
\end{tabular}

For $K^{0}$ production, the SW parametrization is also used although with a different parametrization than either $\pi^{+}$and $\pi^{-}$. The $c_{1}, . ., c_{9}$ parameters come from BNL E910 [47] data and measurements from KEK [49] and are listed in Table 4.3. Due to a dearth of measurement data, $K^{0}$ production simulation is given a conservative $100 \%$ normalization uncertainty.

Table 4.3: SW fit parameters for $K^{0}$ production from BNL E910 and KEK data.

\begin{tabular}{c|c|c|c|c|c|c|c|c|c}
\hline \hline & $\mathrm{c} 1$ & $\mathrm{c} 2$ & $\mathrm{c} 3$ & $\mathrm{c} 4$ & $\mathrm{c} 5$ & $\mathrm{c} 6$ & $\mathrm{c} 7$ & $\mathrm{c} 8$ & $\mathrm{c} 9$ \\
\hline$K^{0}$ & 15.13 & 1.975 & 4.084 & 0.9277 & 0.7306 & 4.362 & 0.0479 & 13.3 & 1.278 \\
\hline \hline
\end{tabular}

For $K^{-}$production, the MARS hadronic interaction package [50 is used to determine the absolute double differential cross section due to lack of measurement data. More details on the meson production simulation and their uncertainties can be found in Reference [3].

\subsubsection{Simulation of Meson Decays}

The mesons undergo the simulated focusing and de-focusing done by the magnetic field from the magnetic horn. The magnetic field has a $1 / r$ dependence from the inner conductor out to the outer radius. The simulation is based on measurements of the magnetic field as shown in Figure 4.4. Since the current in the magnetic horn is pulsed, some charge can bleed beneath the surface of the conductor, which is known as the "skin effect". The current density is modeled to be an exponential decay into the inner conductor with a depth of 1.4 $\mathrm{mm}$ to take into account the skin effect. The uncertainty on the skin effect is taken to be $\pm 1.4 \mathrm{~mm}$, the difference between the default skin effect model and the model where no skin 
effect exists (charge only on the surface of magnetic horn). The magnetic horn current is also given a $\pm 1 \mathrm{kA}$ uncertainty in the simulation.

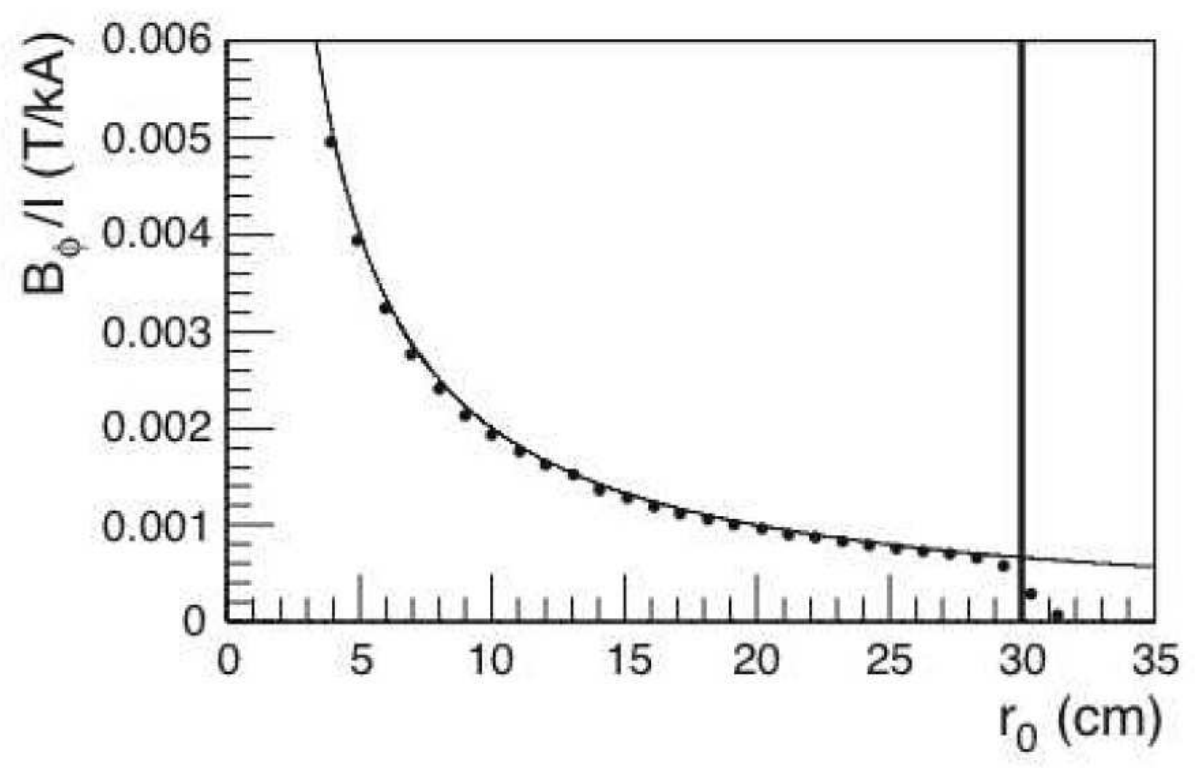

Figure 4.4: Measurements of the azimuthal magnetic field within the horn. The points show the measured magnetic field, while the line shows the expected $1 / r$ dependence. The black vertical line indicates the outer radius of the horn.

After magnetic focusing and de-focusing, the output of the meson production simulation is then used as input for the FORTRAN-based MC code that simulates the neutrino and antineutrino kinematic distributions from meson decays (and subsequent daughter muon decays). Current best knowledge meson and muon decay branching ratios, lifetimes, and decay form factors in three-body semi-leptonic decays are used 51. Polarization effects in muon decays are also accounted for. The neutrinos and antineutrinos produced by the meson decays (and subsequent daughter muon decays) are extrapolated along straight line vectors toward both the SciBooNE and MiniBooNE detectors.

To minimize the MC statistical uncertainties from meson decays, the decay process is simulated multiple times for each meson. Since the decay process is random, the neutrino or antineutrino kinematics resulting from the appropriate meson decay are different each time. For MiniBooNE, each meson is decayed 1000 times. For SciBooNE, each meson 
is decayed 10 times. The smaller multiple number of decays for SciBooNE is due to the larger acceptance of SciBooNE relative to MiniBooNE (SciBooNE is much closer to the decay tunnel than MiniBooNE). Studies also show that the statistical uncertainties does not decrease if the number of decay times is increased past 10 for SciBooNE.

The resulting antineutrino flux at MiniBooNE are all the neutrino and antineutrino vectors that pass through a $610 \mathrm{~cm}$ radius sphere centered at $541 \mathrm{~m}$ downstream the Beryllium target, the location of the MiniBooNE detector. The resulting antineutrino flux at SciBooNE are all the neutrino and antineutrino vectors that pass through a $212 \mathrm{~cm}$ radius sphere centered at $100 \mathrm{~m}$ downstream the Beryllium target, the location of the SciBooNE detector. Figure 4.5 shows the MC neutrino and antineutrino flux prediction in antineutrino mode at both the MiniBooNE and SciBooNE detectors. The antineutrino fluxes at the two detectors are similar, with differences arising from size and acceptance of the detectors. More information on the antineutrino flux can be found in Reference [3].

\subsection{Neutrino and Antineutrino Interactions}

Neutrinos and antineutrinos can interact with other particles only through the weak interaction. Interactions with neutrinos (antineutrinos) mediated through $W^{ \pm}$are known as charged current (CC) interactions. Interactions with neutrinos (antineutrinos) mediated through $Z^{0}$ are known as neutral current (NC) interactions. Almost all the interactions relevant to this analysis are $\mathrm{CC}$ interactions.

The simulation of the neutrino and antineutrino interactions from the antineutrino beam on the MiniBooNE and SciBooNE nuclear targets is performed using the NUANCE software, a program with calculations based off of theoretical models verified and tuned to previous world data. Figure 4.6 shows the relevant neutrino CC cross sections for data and default NUANCE simulation prediction. Figure 4.7 shows the relevant antineutrino CC cross sections for data and default NUANCE simulation prediction. The NUANCE simulation used in this analysis is further tuned based on analyses done by MiniBooNE and neutrino cross section measurements [52]. The MC neutrino and antineutrino interaction simulation is implemented not only for the MiniBooNE and SciBooNE detectors but also 


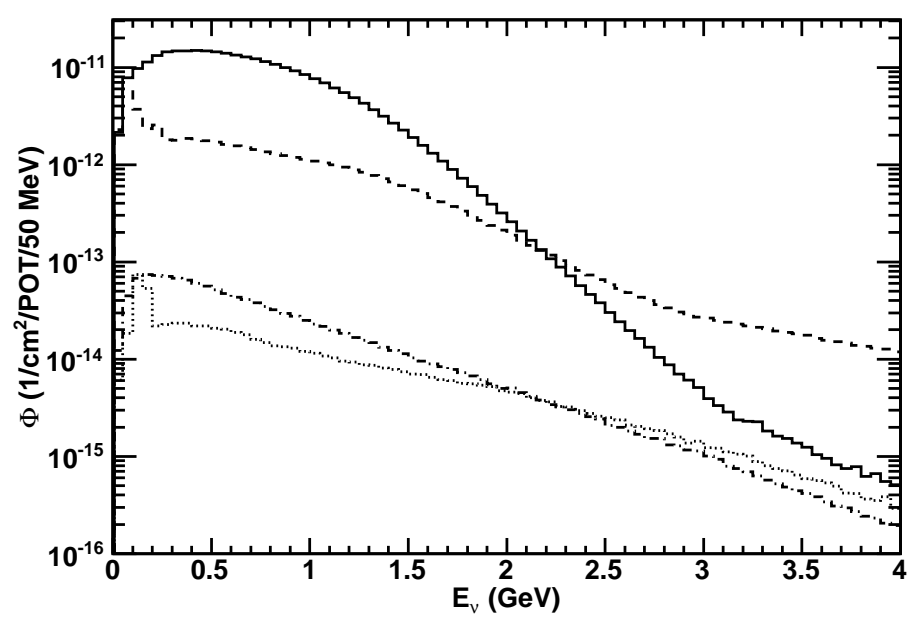

(a) MiniBooNE antineutrino mode flux

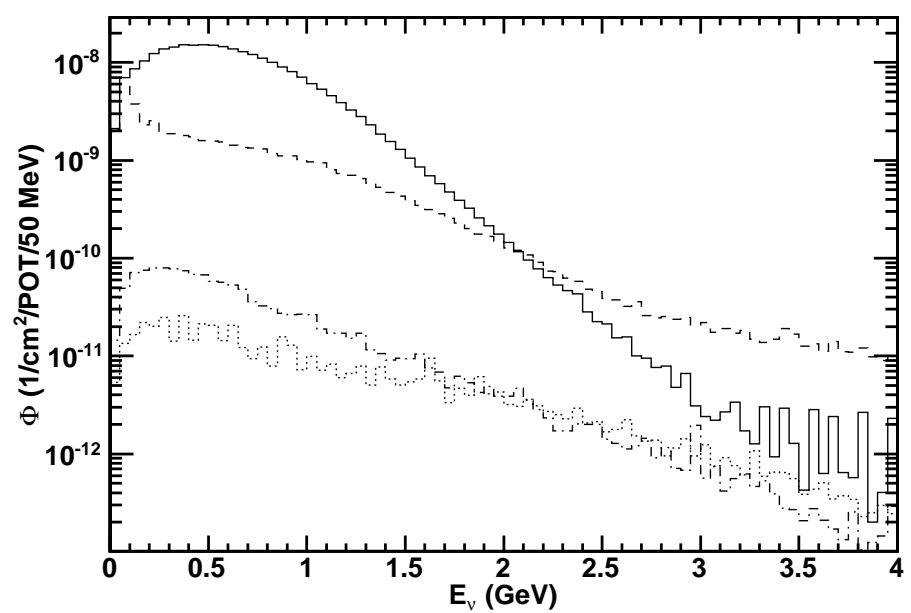

(b) SciBooNE antineutrino mode flux

Figure 4.5: The neutrino and antineutrino flux prediction as a function of true neutrino(antineutrino) energy, in antineutrino mode at the MiniBooNE and SciBooNE detectors. The $\bar{\nu}_{\mu}$ flux is represented by the solid line, the $\nu_{\mu}$ flux is represented by the dashed line, the $\bar{\nu}_{e}$ flux is represented by the dot-dashed line, and the $\nu_{e}$ flux is represented by the dotted line. 
in their surrounding materials to better understand and estimate backgrounds.

Almost all CC neutrino (antineutrino) interactions can roughly be divided into three categories, each relevant and dominating within a certain neutrino (antineutrino) energy range. In order of ascending neutrino (antineutrino) energy, the three categories are: quasielastic, meson production (mostly consisting of single pion production), and deep inelastic scattering (DIS).

\subsubsection{Quasielastic Scattering}

The most common neutrino and antineutrino interaction type for the analysis is charged current quasielastic (CCQE) scattering. It dominates at neutrino (antineutrino) energies of about $1 \mathrm{GeV}$. For neutrinos, the interaction process is $\nu_{l}+n \rightarrow l^{-}+p$ where $l$ is the lepton flavor. For antineutrino, the interaction process is $\bar{\nu}_{l}+p \rightarrow l^{+}+n$ where $l$ is the lepton flavor. CCQE scattering produces a clean, distinct signal and relatively easy to simulate and reconstruct.

The CCQE cross section was first described by Llwellyn-Smith 74:

$$
\frac{d^{2} \sigma C C Q E_{\nu(\bar{\nu})}}{d Q^{2}}=\frac{M^{2} G_{F}^{2} \cos ^{2}\left(\theta_{c}\right)}{8 \pi E_{\nu(\bar{\nu})}^{2}}\left[A\left(Q^{2}\right) \pm B\left(Q^{2}\right) \frac{s-u}{M^{2}}+C\left(Q^{2}\right) \frac{(s-u)^{2}}{M^{4}}\right]
$$

where $M$ is the nucleon mass, $G_{F}$ is the Fermi coupling (strength of the weak force), $\theta_{c}$ is the Cabbibo angle, $s$ and $u$ are Mandelstam variables with $s-u=4 M E_{\nu}-Q^{2}-m_{l}, Q^{2}$ is the 4-momentum transferred between the neutrino (antineutrino) and the outgoing lepton. The derivation can be found in Reference 75].

$A, B$, and $C$ are functions of $Q^{2}$ defined as:

$$
\begin{gathered}
A\left(Q^{2}\right)=\frac{m^{2}+Q^{2}}{M^{2}}\left[(1+\tau) F_{A}^{2}-(1-\tau) F_{1}^{2}+\tau(1-\tau) F_{2}^{2}+4 \tau F_{1} F_{2}\right. \\
\left.-\frac{m^{2}}{4 M^{2}}\left(F_{1}+F_{2}\right)^{2}\left(F_{A}+2 F_{p}\right)^{2}-4 F_{p}^{2}(1+\tau)\right] \\
B\left(Q^{2}\right)=\frac{Q^{2}}{M^{2}} F_{A}\left(F_{1}+F_{2}\right) \\
C\left(Q^{2}\right)=\frac{1}{4}\left(F_{A}^{2}+F_{1}^{2}+\tau F_{2}^{2}\right)
\end{gathered}
$$




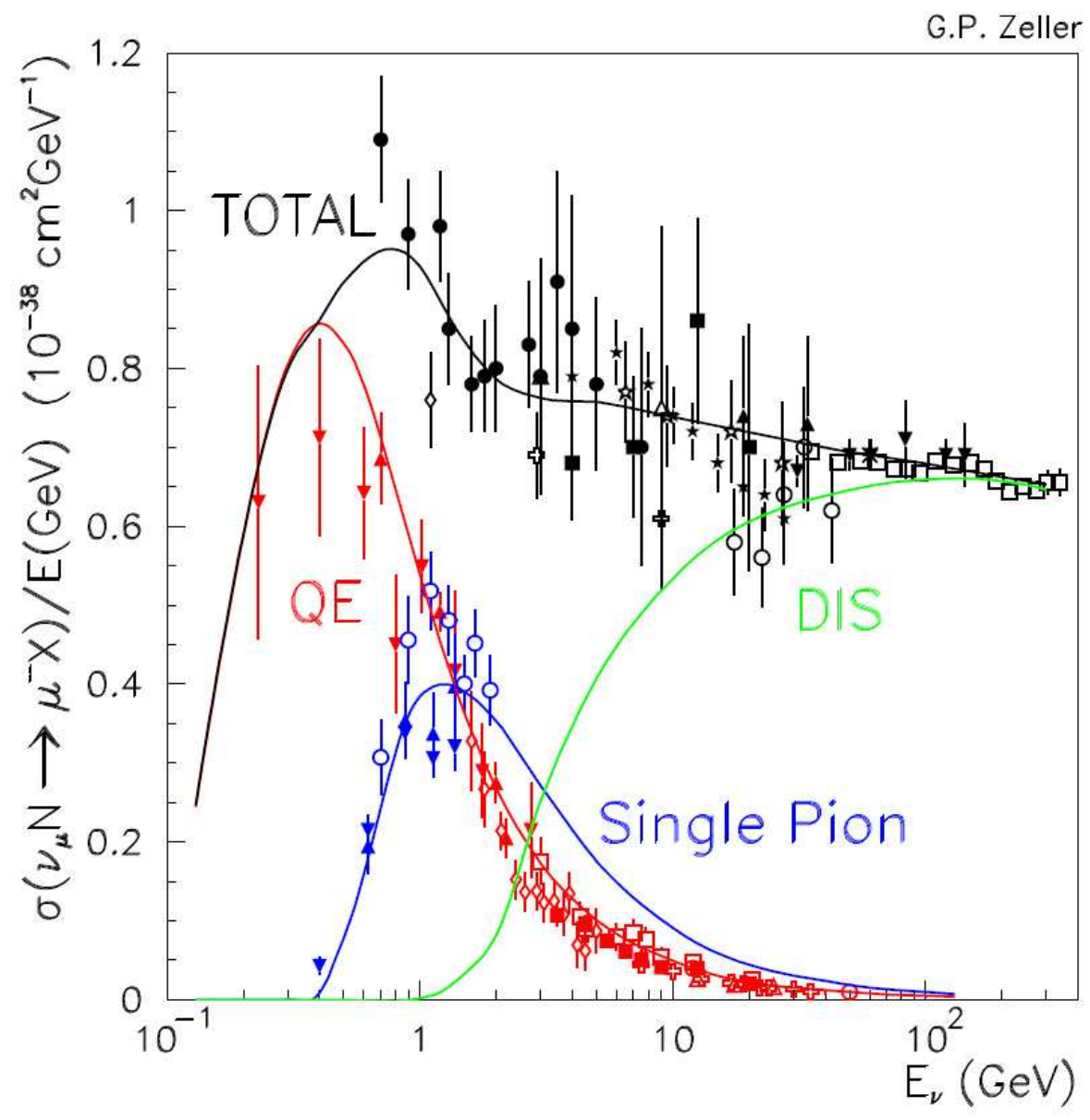

Figure 4.6: Previous world published data $53,54,55,56 ; 57,58,59 ; 60 ; 61 ; 62 ; 63 ; 64$; 65, 66, 67; 68; 69 on neutrino CC cross sections. The CC cross sections are separated by interaction type and divided by neutrino energy and plotted as a function of neutrino energy. Charged current quasielastic interaction is shown in red. Charged current single pion interactions are shown in blue. Deep inelastic scattering interactions are shown in green. Total CC cross sections are shown in black. The colored points are the data with uncertainties and the colored solid lines are the default NUANCE simulation predictions before further tuning based on MiniBooNE analyses. (From Reference 70])

where $\tau=\frac{Q^{2}}{4 M^{2}}, F_{1}$ and $F_{2}$ are the vector form factors, $F_{A}$ is the axial vector form factor, and $F_{p}$ is the pseudo scalar form factor. $m$ is the lepton mass. 


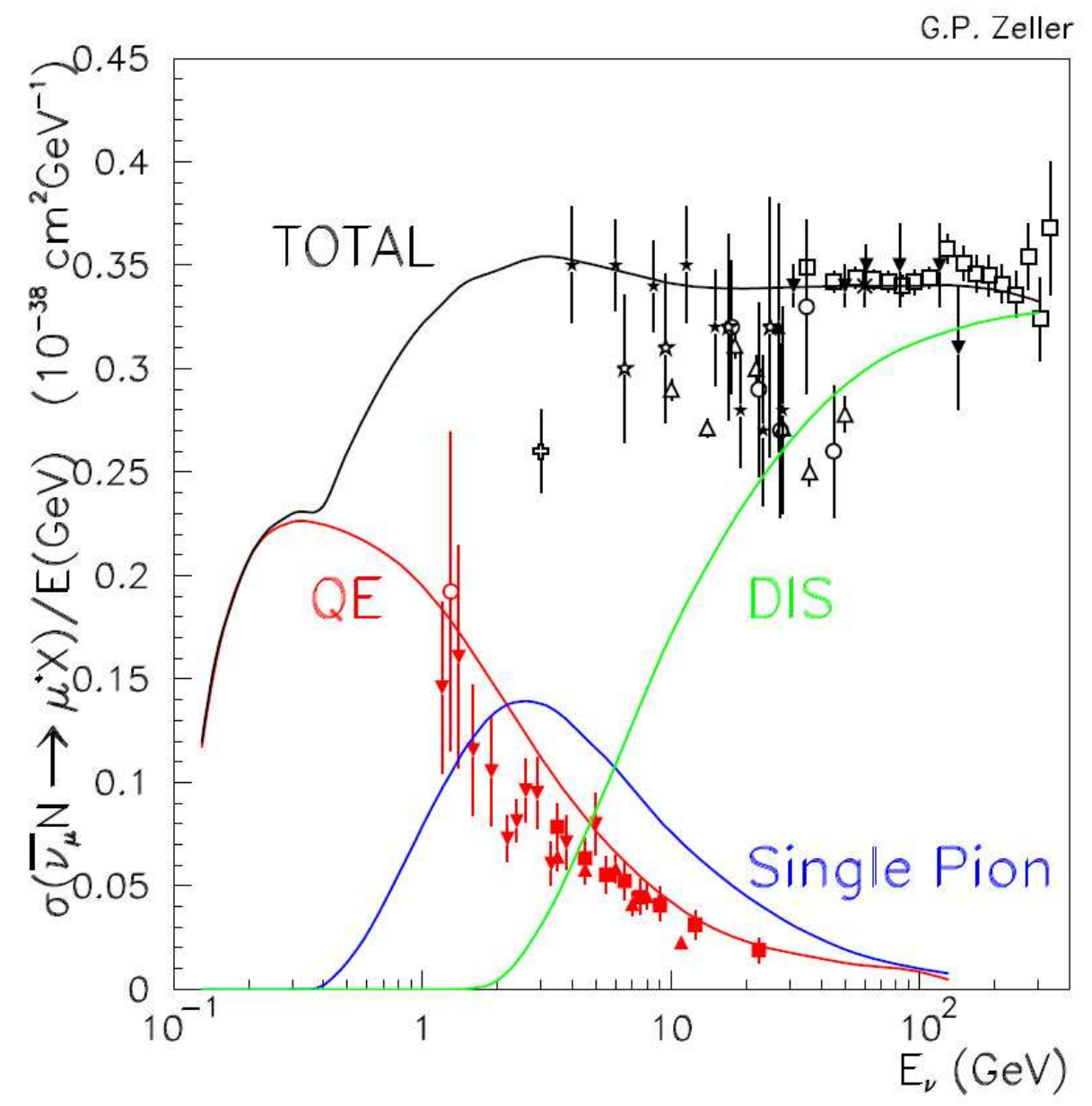

Figure 4.7: Previous world published data $62 ; 63 ; 64 ; 65 ; 66 ; 67 ; 68 ; 69 ; 71 ; 72,73$ on antineutrino CC cross sections. The CC cross sections are separated by interaction type and divided by antineutrino energy and plotted as a function of antineutrino energy. Charged current quasielastic interaction is shown in red. Charged current single pion interactions are shown in blue. Deep inelastic scattering interactions are shown in green. Total CC cross sections are shown in black. The colored points are the data with uncertainties and the colored solid lines are the default NUANCE simulation predictions before further tuning based on MiniBooNE analyses. (From Reference [70])

Assuming conserved vector current (CVC), the vector form factors $F_{1}$ and $F_{2}$ are defined 
as:

$$
\begin{gathered}
F_{1}\left(Q^{2}\right)=\frac{1}{1+\tau}\left(G_{E}^{p}-G_{E}^{n}-\tau\left(G_{M}^{p}-G_{M}^{n}\right)\right) \\
F_{2}\left(Q^{2}\right)=\frac{1}{1+\tau}\left(G_{M}^{p}+G_{M}^{n}-G_{E}^{p}+G_{E}^{n}\right)
\end{gathered}
$$

where $G_{M, E}^{p, n}$ are the Sachs form factors present in electron scattering. Conventionally, the $G_{M, E}^{p, n}$ form factors are assumed to be in a dipole format:

$$
G_{E, M}^{p, n}\left(Q^{2}\right)=\frac{G_{E, M}^{p, n}(0)}{1+\frac{Q^{2}}{M_{V}^{2}}}
$$

where $G_{E}^{p}(0)=1$ is the proton electric charge, $G_{E}^{n}(0)=0$ is the neutron electric charge, $G_{M}^{p}(0)=2.793$ is the proton magnetic moment, and $G_{M}^{n}(0)=-1.913$ is the neutron magnetic moment. The vector mass is $M_{V}^{2}=0.71 \mathrm{GeV}^{2}$.

Recent electron scattering data show deviations from the dipole format at high $Q^{2}$ so these form factors are parameterized as functions of Q2 from fits 76 to electron scattering data $77 ; 78 ; 79 ; 80$.

The axial form factor $F_{A}$ is assumed to be in a dipole format:

$$
F_{A}\left(Q^{2}\right)=\frac{-1.267}{1+\frac{Q^{2}}{M_{A}^{2}}}
$$

where $M_{A}$ is the axial mass for the CCQE interaction. $M_{A}$ is a tunable parameter and for the NUANCE simulation of this analysis, $M_{A}$ is based on the latest MiniBooNE muon neutrino cross section measurement 52$]$ and set to be $1.35 \pm 0.07 \mathrm{GeV} / \mathrm{c}^{2}$ for carbon targets. Figure 4.8 shows the MiniBooNE muon neutrino CCQE cross section per neutron as a function of neutrino energy with the best fit RFG model of $M_{A}=1.03 \mathrm{GeV} / \mathrm{c}^{2}$ and $\kappa=1.000$ (among other models). $M_{A}$ for hydrogen targets is set to $1.014 \pm 0.014 \mathrm{GeV} / \mathrm{c}^{2} 81$.

The pseudo scalar form factor $F_{p}$ is defined as:

$$
F_{p}\left(Q^{2}\right)=\frac{2 M^{2}}{m_{\pi}^{2}+Q^{2}} F_{A}\left(Q^{2}\right)
$$

where $m_{\pi}$ is the mass of the pion. More information can be found in Reference 75 .

The CCQE cross section model by Llwellyn-Smith assumes that the neutrino (antineutrino) interacts with a neutron (proton) while in reality, the neutrino (antineutrino) mainly 

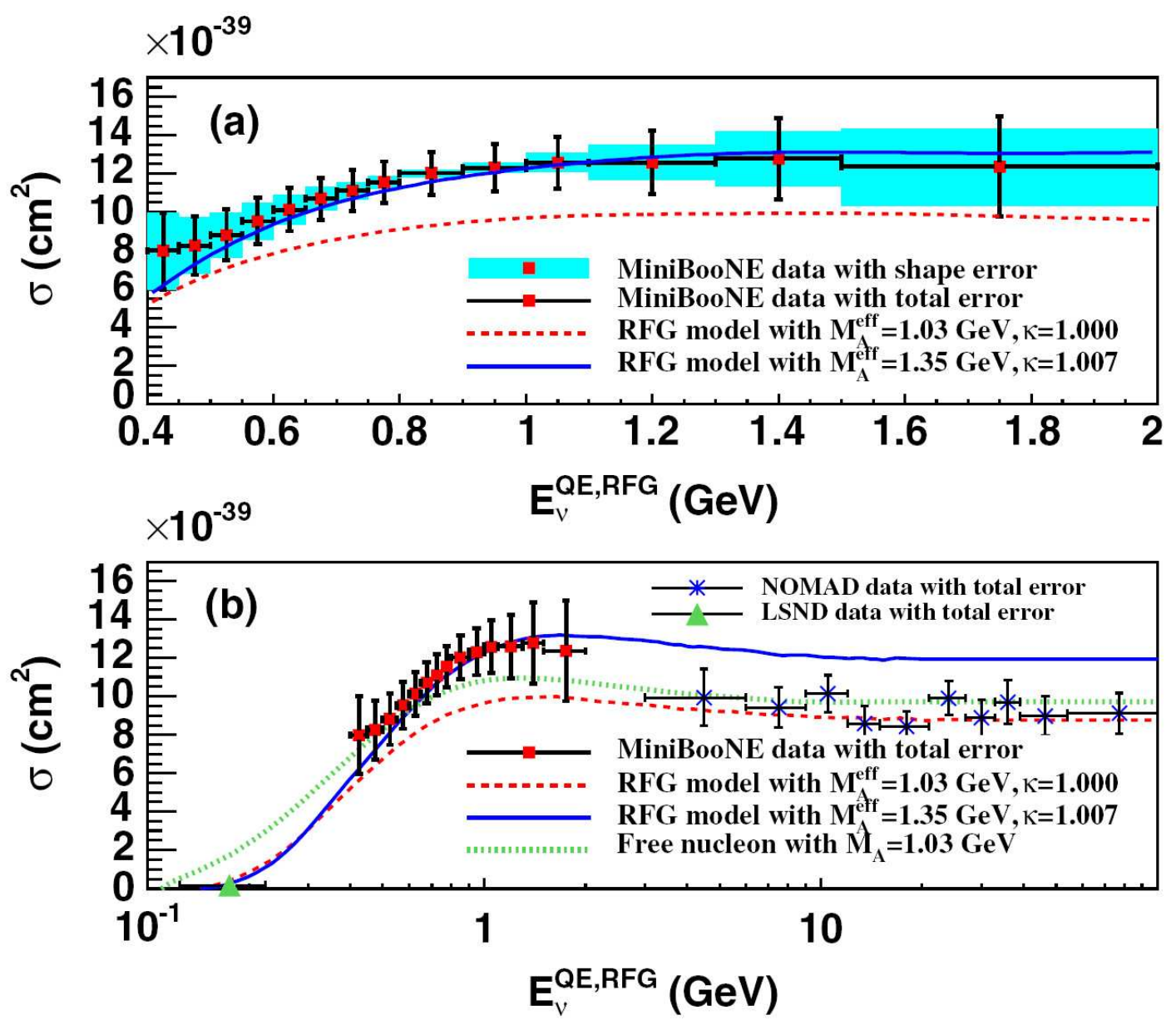

Figure 4.8: MiniBooNE muon neutrino CCQE cross section per neutron as a function of neutrino energy. In (a), shape errors are shown as shaded boxes along with the total errors as bars. In (b), a larger energy range is shown along with results from the LSND [82] and NOMAD [83] experiments. Also shown are predictions from the NUANCE simulation for an RFG model with $M_{A}=1.35 \mathrm{GeV} / \mathrm{c}^{2}$ and $\kappa=1.007$, for an RFG model with $M_{A}=1.03$ $\mathrm{GeV} / \mathrm{c}^{2}$ and $\kappa=1.000$, and for scattering from free nucleons with the world-average $M_{A}$ value. (From Reference [52])

interacts with the carbon targets of MiniBooNE and SciBooNE. The modeling of this nuclear effect was first derived by Smith and Moniz 84. The formula includes hadronic nuclei functions which depend on the form factors and the distribution $f$ of the nuclei in 
momentum space $\left(\int d \vec{k} f(\vec{k}, \vec{q}, \omega)\right)$.

The carbon target is modeled to be a relativistic Fermi Gas (RFG) characterized by Fermi momentum $p_{F}$ and nucleon binding energy $E_{B}$. The integral then becomes:

$$
\int d \vec{k} f(\vec{k}, \vec{q}, \omega)=\int_{E_{\text {low }}}^{E_{h i}} E d E
$$

where $E_{l o w}=\kappa \sqrt{p_{F}^{2}+M_{n}^{2}}$ and $E_{h i}=\kappa \sqrt{p_{F}^{2}+M_{p}^{2}}-\omega+E_{B}$. Based on previous MiniBooNE analysis [85], the factor $\kappa$ is introduced and represents a scale factor for the allowed momentum space and can increase or decrease the amount of Pauli blocking. $\kappa$ is introduced to allow an extra degree of freedom to describe MiniBooNE data at low $Q^{2}$. The simulation sets $E_{B}=30 \pm 9 \mathrm{MeV}$ for protons in carbon and $p_{F}=220 \pm 30 \mathrm{MeV} / \mathrm{c}$ in carbon, which are based from electron scattering data.

\subsubsection{Meson Production}

At higher neutrino and antineutrino energies, meson production occurs during the scattering. There are two channels for this process: resonance production and coherent production. $\pi, K$, and $\eta$ resonance meson production through neutrino or antineutrino interactions are modeled using the Rein and Seghal formalism 86. Coherent $\pi$ production is also modeled by Rein and Seghal $87 ; 88$.

The Rein and Seghal resonance production model assumes an intermediate baryon resonance, $N *$, in the reaction of $\nu_{l}+p / n \rightarrow l^{-}+N *$ and $N * \rightarrow N^{\prime}+p / n$ for neutrinos and $\bar{\nu}_{l}+p / n \rightarrow l^{+}+N *$ and $N * \rightarrow N^{\prime}+p / n$ for antineutrinos, where $N^{\prime}$ is the emitted $\pi, K$, or $\eta$. All intermediate baryon resonances with mass less than $2 \mathrm{GeV} / \mathrm{c}^{2}$ are included. Baryon resonances with mass greater than $2 \mathrm{GeV} / \mathrm{c}^{2}$ are simulated as deep inelastic scattering. For the $\Delta(1232)$ resonance, the Rein and Seghal method 89 is used to determine the angular distribution of the final state pions. For all other resonances, the angular distribution of the generated pion is chosen to be isotropic in the resonance rest frame. For the simulation, the tuneable parameter for resonance production $M_{A}^{r e s} \pi$ is set to be $1.1 \pm 0.275 \mathrm{GeV} / \mathrm{c}^{2}$ for all targets.

Coherent $\pi$ production assumes that the neutrino (antineutrino) scatters off the entire nuclear target in the process $\nu_{l}+X \rightarrow l^{-}+\pi^{+}+X^{\prime}\left(\bar{\nu}_{l}+X \rightarrow l^{+}+\pi^{-}+X^{\prime}\right)$ where $X$ is the 
nuclear target before and $X^{\prime}$ is the nuclear target after the scattering. For the simulation, the tuneable parameter for coherent production $M_{A}^{c o h \pi}$ is set to be $1.03 \pm 0.275 \mathrm{GeV} / \mathrm{c}^{2}$ for all targets. Coherent $\pi$ production plays a very small contribution to the analysis.

The simulation includes an additional adjustment to all CC single $\pi$ production events from neutrino and antineutrino interactions. This adjustment is performed as a function of $Q^{2}$ to correct for the discrepancies noticed between data and MC in both SciBooNE and MiniBooNE for CC single $\pi$ production events [52; 90. It is based on measurements in MiniBooNE using a purified sample of CC single $\pi$ production events 52 .

Meson production leading to multi-pion final states are also included in the simulation and simulated assuming $M_{A}^{N \pi}=1.30 \pm 0.52 \mathrm{GeV} / \mathrm{c}^{2}$ in NUANCE. The value of $M_{A}^{N \pi}$ is chosen to ensure that the total $\mathrm{CC}$ cross section prediction reproduces previous experimental data.

\subsubsection{Deep Inelastic Scattering}

At high neutrino and antineutrino energies, deep inelastic scattering (DIS) occurs. The DIS cross section is calculated using the GRV98 parton distribution functions 91. Additionally, there are corrections in the small $Q^{2}$ region developed by Bodek and Yang [92. The DIS contribution slowly increases for hadronic invariant masses $W$ starting at $1.7 \mathrm{GeV}$ and becomes the only source of neutrino and antineutrino interactions above $2 \mathrm{GeV}$. This is done in NUANCE to create a smooth transition between the resonance single pion production and DIS models and ensure continuity in distributions of kinematics and hadron multiplicity.

\subsubsection{Final State Internuclear Interactions}

In NUANCE, neutrino and antineutrino interactions are modeled as being instantaneous in time. After the initial neutrino or antineutrino interaction, any particles produced from the neutrino or antineutrino interaction (e.g. $p, n, \pi)$ can interact with the carbon nucleus. In NUANCE, the probability for the particle to interact is calculated in discrete $0.3 \mathrm{fm}$ increments until the particle exits the $2.5 \mathrm{fm}$ radius of the carbon nucleus. Pion absorption $\left(\pi^{+/-}+X \rightarrow X^{\prime}\right)$, pion charge exchange $\left(\pi^{+/-}+X \rightarrow \pi^{0}+X^{\prime}\right)$, and pionless $\Delta$ decay $\left(\Delta^{++} \rightarrow \Delta^{+}, \Delta^{+} \rightarrow \Delta\right)$ are also simulated in NUANCE and given uncertainties of $\pm 25 \%$, 
$\pm 30 \%$, and $\pm 100 \%$, respectively.

For SciBooNE, the simulation of these final state internuclear interactions are important because $\pi$ and $p$ emitted from the carbon nucleus can mimic the $\mu$ signal for the analysis. Also, the angle and energy of the $\mu$ can be altered by these internuclear interactions. For MiniBooNE, pion absorption, pion charge exchange, and pionless $\Delta$ decay causes single pion production interactions to resemble CCQE interactions, affecting the signal selection for the analysis.

\subsection{Detector Response}

The simulation of the detector responses for SciBooNE and MiniBooNE are different due to the inherent differences between the detectors (discrete tracking versus Cherenkov).

\subsubsection{MiniBooNE Detector Response}

MiniBooNE uses GEANT3 [93] for simulation of the detector. The detector is simulated to be a sphere with small "top hat"-like structure at the top and filled with mineral oil of the appropriate density based on measurement. Additional simulation places the detector inside a concrete, cylindrical vault filled with air, which is surrounded by dirt with density $2.15 \mathrm{~g} / \mathrm{cm}^{3}$ on all sides and above the detector.

The PMTs are modeled based on their tube type and measured response. The default GEANT3 simulation is used to model most particle propagation in the detector. The GEANT3 code was modified to include a custom model for light propagation in the detector [94]. Scintillation, fluorescence, and Cherenkov light components are all simulated. Absorption, scattering, and reflections in the detector tank and veto are also simulated.

A custom muon capture model is also added to simulate the $7 \%$ of $\mu^{-}$capture on mineral oil. GCALOR [95] is used for the final state hadronic model and models the low energy photons and neutrons produced upon $\mu^{-}$capture. GCALOR is also used to model the pion absorption and charge exchange in the detector medium. Some modifications were made to the standard GCALOR code, namely $\pi^{ \pm}$elastic scattering and photonuclear interactions 96 . 


\subsubsection{SciBooNE Detector Response}

SciBooNE uses GEANT4 97 to simulate the interactions of hadronic particles with detector materials. The SciBar, EC and MRD sub-detectors are all modeled with their respective construction materials and their model positions are based on survey data. The position of the SciBar planes relative to the first layer is set in the simulation based on survey data and cross checked with cosmic ray muon data [51].

For the simulation of the SciBar sub-detector, GEANT4 is used to model the true energy depositions in scintillator strips, which are then converted to the number of photoelectrons at the PMTs. The conversion factors are calculated based on the measured light yields for cosmic muons and the measured gains of PMTs. The scintillator quenching is simulated using Birk's law with the measured value of Birk's constant 44]. The measured light attenuation length is used in the simulation. The crosstalk between the MA-PMT is measured to be $3.15 \pm 0.4 \%$ for adjacent channels and used for the simulation. The single photoelectron resolution of the MA-PMT is set to $50 \%$ in the simulation to reproduce the observed $\mathrm{dE} / \mathrm{dx}$ distribution of cosmic muons and the absolute error is estimated to be $\pm 20 \%$. The density of the scintillator is measured by sampling $10 \%$ of the strips before the installation and determined to be $1.021 \mathrm{~g} / \mathrm{cm}^{3}$ and implemented into the simulation. The uncertainty of the total mass is estimated to be $1 \%$, including the effect of epoxy resin used to glue the strips.

For the simulation of the EC sub-detector, the energy to photoelectron conversion for each channel is simulated based on cosmic ray data and the attenuation length of the fibers is also simulated based on measurement. For the simulation of the MRD sub-detector, the time of energy deposition is digitized and converted into TDC counts. The positions of steel plates and scintillator strips are surveyed and implemented in the simulation. The gaps between the scintillator counters are also simulated.

In addition to simulating neutrino and antineutrino interactions in the detector, the interactions in the surrounding material, including the walls of the detector hall and the soil, are also simulated to estimate neutrino and antineutrino backgrounds. The density of surrounding material is assumed to be $2.15 \mathrm{~g} / \mathrm{cm}^{3}$ for the simulation. Surrounding material $\mathrm{MC}$ events a distance $\pm 5 \mathrm{~m}$ away in all three coordinate direction from the SciBooNE detector are generated and included in the event predictions. 


\section{Chapter 5}

\section{Event Reconstruction and}

\section{Selection}

The purpose of reconstruction and selection is to identify the particles interacting in the detectors given the raw detector signals and to efficiently select samples of events with the desired particles, which are $\mu^{+}$in this analysis, with low background event contamination. The selection criteria are chosen with a balance to maximize the number of background event rejected and to preserve the largest number of desired $\mu^{+}$events. This balance requires that different selection criteria are used for MiniBooNE and SciBooNE. MC simulation of the data is used to understand the properties of the selected samples. The same selection criteria are applied to both data and MC so that the selected MC samples can be compared directly to their corresponding data samples and used to estimate the effectiveness of the reconstruction on the data, i.e. the purity and efficiency of the selection criteria on data and the properties of the selected data samples. Ultimately, the selected data and MC samples will be used to test the theory proposed in this analysis.

The chapter is organized as follows. SciBooNE and MiniBooNE event reconstruction are first discussed, followed by information on the data sets used. Then, SciBooNE and MiniBooNE event selection are described followed by antineutrino energy reconstruction. The energy distributions will ultimately be used in the fit to test the oscillation hypothesis. Finally, the properties and composition of the selected samples are presented and the chapter 
is concluded with a summary of the results.

\subsection{SciBooNE Event Reconstruction}

SciBooNE is a discrete tracking detector so event reconstruction involves combining into tracks the hits from the passage of charged particles through the components of the detector. The length, position, and energy deposited in the tracks are assumed to correspond with the charged particle passing through the detector. Tracks are first formed by looking at adjacent hits in SciBar, the most sensitive sub-detector of SciBooNE. The tracks in SciBar are then matched to hits in the EC and reconstructed tracks or hits in the MRD.

\subsubsection{SciBar Track Reconstruction}

A SciBar hit is a signal event where more than two photoelectrons, or approximately 0.2 $\mathrm{MeV}$, is deposited in a scintillator strip. Associated hits in either the $\mathrm{X}-\mathrm{Z}$ or $\mathrm{Y}-\mathrm{Z}$ planes of SciBar are combined using a cellular automaton algorithm [98 to form two-dimensional SciBar tracks. The cellular automaton algorithm was first developed for the K2K experiment. SciBar hits are organized into clusters, where each cluster consists of adjacent SciBar hits. Segments are formed that connect the individual clusters no more than one scintillator strip apart. Any segments that share a cluster are then connected if the $\chi^{2}$ of a least squares linear fit to the segments is within range.

Three-dimensional SciBar tracks are reconstructed by matching the timing and edges in the z-direction of two two-dimensional SciBar tracks, one in the X-Z plane and one in the Y-Z plane. Time differences between two two-dimensional SciBar tracks are required to be less than $50 \mathrm{~ns}$, and the distance between the edges in the z-direction must be less than 6.6 $\mathrm{cm}$ for both upstream and downstream edges. If more than one two-dimensional track in one plane is matched up with another two-dimensional track in the other plane, the combination with the smallest summed value of the distances between their respective upstream and downstream edges in the z-direction is chosen. Three-dimensional reconstructed SciBar tracks have a minimum length of roughly $8 \mathrm{~cm}$. 


\subsubsection{EC Cluster Reconstruction}

An EC cluster is formed by taking adjacent hits in the EC for either the $\mathrm{X}-\mathrm{Z}$ or $\mathrm{Y}-\mathrm{Z}$ plane. The energy of the EC cluster is the sum of the energy of the EC hits and the position of the EC cluster is the energy weighted average of its composed EC hits. The EC cluster was developed to reconstruct photons and electrons and therefore not used in this analysis. The EC energy is estimated using EC track length in this analysis.

\subsubsection{MRD Track Reconstruction}

Two-dimensional tracks in the MRD are independently reconstructed using MRD hits in either the X-Z or Y-Z plane clustered within a $50 \mathrm{~ns}$ timing window. Three-dimensional tracks in the MRD are reconstructed by matching the timing of the two-dimensional MRD tracks. At least two MRD layers in each view are required to reconstruct a MRD track, resulting in a minimum penetration of three steel layers.

\subsubsection{SB-MRD Matched Track}

For this analysis, certain charged particles are produced in the fiducial volume of SciBar and stop in the fiducial volume of MRD so additional reconstruction between a reconstructed three-dimensional SciBar track and associated MRD hits or three-dimensional track is necessary. Such a track is defined as a SB-MRD matched track. No EC information is used in constructing the SB-MRD matched track.

A search for a candidate SciBar track that has a downstream $z$-direction edge that lies in the two most downstream layers of SciBar is first conducted. If the candidate SciBar track exists and there is also a MRD track, then several additional criteria must be fulfilled. The upstream edge of the MRD track must be within the two most upstream layers of the MRD, and be within $30 \mathrm{~cm}$ of the projected entry point of the candidate SciBar track into the MRD in both the $\mathrm{X}-\mathrm{Z}$ and $\mathrm{Y}-\mathrm{Z}$ directions. The maximum difference between the SciBar and MRD track angles with respect to the beam direction is given by $\theta_{\max }=\left|\theta_{S B}-\theta_{M R D}\right|$, with $\theta_{\max }$ required to be below a certain threshold, which varies between 0.4 radian and 1.1 radians, depending on the length of the MRD track. If no MRD track is found, the candidate SciBar track is extrapolated to the MRD to search for nearby MRD hits. The nearby MRD 
hits are required to be within a cone with an aperture of \pm 0.5 radian and a transverse offset within $10 \mathrm{~cm}$ of the extrapolated candidate SciBar track at the upstream edge of the MRD. The timing difference between the candidate SciBar track and the MRD track or hits must be within $100 \mathrm{nsec}$. The matching criteria imposes a minimum momentum of $350 \mathrm{MeV} / \mathrm{c}$ for the SB-MRD matched track.

\subsection{MiniBooNE Event Reconstruction}

MiniBooNE is a Cherenkov detector so event reconstruction involves searching for associated PMT activity generated from Cherenkov light created as charged particles pass through its mineral oil volume. The data stream of the PMT activity is divided into "events", where a single "event" is all the time and charge information for all the PMTs in a 19.2 $\mu$ s DAQ time window. Each event can consists of separate "subevents", or clusters of PMT activity separated in location and time. A subevent must have at least 10 PMT hits where no two consecutive hits are separated in time by more than $10 \mathrm{~ns}$.

Event reconstruction involves converting the spatial patterns in charge and time information obtained by the PMTs into the momentum and location information of the passing particles. The passing particles are assumed to create tracks in the mineral oil volume and each interaction is modeled as either a single track (CCQE $\nu_{\mu}, \bar{\nu}_{\mu}, \nu_{e}, \bar{\nu}_{e}$ interaction events, cosmic muons, decay electrons) or two tracks (NC $\pi_{0}$ interaction events). For CC events with an associated $\pi^{+}$or $\pi^{-}$production, the $\pi^{+}$or $\pi^{-}$decays immediately to a $\mu^{+}$or $\mu^{-}$, respectively, which then decays into an $e^{+}$or $e^{-}$. The $e^{+}$or $e^{-}$are classified as a different subevent. For CC events with an associated $\pi^{0}$ production, the $\pi^{0}$ is classified as another subevent different from the subevent of the charged particle produced in the CC interaction. Each subevent is characterized by a set of PMT signals $q_{i}$ and $t_{i}$ which are determined by an underlying set of parameters $\vec{a}$ which characterize the particle's trajectory and properties. The parameters of $\vec{a}$ are starting position $\left(x_{0}, y_{0}, z_{0}\right)$, starting time $t_{0}$, direction $\left(\theta_{0}, \phi_{0}\right)$, and kinetic energy $E_{0}$.

For the single track model, the likelihood of a set of parameters $\vec{a}$ given $q_{i}$ and $t_{i}$ is determined by the single track likelihood equation $L$ : 


$$
L=\prod_{i=\text { unhit } P M T}^{\text {all unhit } P M T s}(1-P(i \text { hit } ; \vec{a})) \prod_{i=h i t ~}^{\text {all hit } P M T} P(i \text { hit } ; \vec{a}) L_{q}\left(q_{i}, \vec{a}\right) L_{t}\left(t_{i}, \vec{a}\right)
$$

where $P(i$ hit; $\vec{a})$ is the probability that the $i$-th PMT is hit given parameters $\vec{a}$ and $L_{q}$ and $L_{t}$ are the probabilities of measuring charge $q$ at time $t$ in PMT $i$ due to parameters $\vec{a}$. The optimal values of parameters $\vec{a}$ are found by maximizing $L$ for the event.

The charge likelihood $L_{q}$ is determined in a two step process. In the first step, using the known optical photon and particle propagation properties of the mineral oil, given the particle type $\left(e^{-} / e^{+}\right.$or $\left.\mu^{-} / \mu^{+}\right)$and parameters $\vec{a}$, the average number of photoelectrons that a particular PMT should observe can be determined. In the second step, the PMT response to the photoelectrons observed is determined. The PMT response model was established using laser calibration data.

The time likelihood $L_{t}$ is determined with a combination of a time component from the particle's Cherenkov light, which follows a Gaussian distribution, and a time component from the particle's scintillation light, which has a exponential decay.

For the two track model, the two track likelihood equation has the same form as the single track likelihood equation, but with different calculations for the charge likelihood $L_{q}$ and the time likelihood $L_{t}$. $L_{q}$ is determined by adding the predicted charges from the two tracks to obtain the total predicted charge at each PMT. $L_{t}$ is determined by a weighted time average of the Cherenkov and scintillation time components from the two tracks. More details on MiniBooNE event reconstruction can be found in Reference 99 .

\subsection{SciBooNE and MiniBooNE Data Sets}

The Booster Neutrino Beam (BNB) operated from February 2003 till April 2012 and delivered both neutrinos and antineutrinos to the MiniBooNE and SciBooNE detectors. The amount of data collected from the BNB is measured in protons on target (POT), or the amount of protons delivered to the Be target. During the time period, the magnetic horn was sometimes configured in neutrino mode running, which provided a neutrino-rich beam, and sometimes configured in antineutrino mode running, which provided an antineutrinorich beam to both MiniBooNE and SciBooNE detectors. 


\subsubsection{BNB POT Collection}

Table 5.1 shows the POT collected for both experiments over their entire data collection periods. During the first antineutrino mode running collection period, absorber plates fell into the BNB decay volume altering the antineutrino flux. This period occurred from June 2006 to April 2007 and the antineutrino data during this period is excluded in the analysis. Excluding the downed absorber plates period, $1.010 \times 10^{21}$ POT was collected in antineutrino mode running for MiniBooNE and used for the analysis. The downed absorber plates period did not occur during SciBooNE data taking so the entire antineutrino mode running data set for SciBooNE $\left(1.53 \times 10^{20}\right.$ POT total $)$ is used for the analysis.

\begin{tabular}{|c|c|c|c|}
\hline Period & BNB Mode & SB POT & MB POT \\
\hline Feb. 2004-Jan. 2006 & neutrino & - & $5.58 \times 10^{20}$ \\
\hline Jan. 2006-Oct. 2007 & antineutrino & $\begin{array}{l}0.52 \times 10^{20} \\
\text { (Jun. } 2007 \\
\text {-Aug. 2007) }\end{array}$ & $\begin{array}{l}1.71 \times 10^{20} \\
\left(1.18 \times 10^{20} \text { were used in the }\right. \\
\text { analysis corresponding to } \\
\text { Jan. } 2006 \text {-May } 2006 \text { and } \\
\text { May } 2007-\text { Oct. } 2007 \text { when } \\
\text { all absorber plates were } \\
\text { correctly positioned) }\end{array}$ \\
\hline Oct. 2007-Apr. 2008 & neutrino & $0.99 \times 10^{20}$ & $0.83 \times 10^{20}$ \\
\hline Apr. 2008-Apr. 2012 & antineutrino & $\begin{array}{l}1.01 \times 10^{20} \\
(- \text { Aug. } 2008)\end{array}$ & $8.92 \times 10^{20}$ \\
\hline
\end{tabular}

Table 5.1: Summary of data run periods for SciBooNE and MiniBooNE.

\subsubsection{Data Quality}

To ensure that the data collected from detectors are accurate, data quality checks are imposed for both MiniBooNE and SciBooNE throughout their entire run periods. Only data that pass the given detector's respective quality checks are included in the analysis. 


\subsubsection{SciBooNE Data Quality Checks}

For SciBooNE, the data must pass five quality checks: Proton Beam Intensity, Toroid Agreement, Peak Horn Current, Targeting Efficiency, and GPS Time Difference.

Proton Beam Intensity: The intensity of the primary proton beam is measured on a spill-by-spill basis using TOR875, a torodial current transformer. Since the proton beam typically contains $5 \times 10^{12}$ protons per pulse, at least $0.1 \times 10^{12}$ protons per pulse are required per beam spill.

Toroid Agreement: To ensure the proton beam is properly transported, the fractional difference between two torodial current transformers, TOR860 and TOR875, located at two different positions along the beamline must be within $10 \%$.

Peak Horn Current: For accurate magnetic field focusing of the charged particles in the beamline, the absolute value of the peak magnetic horn current must be above $170 \mathrm{kA}$.

Targeting Efficiency: The targeting efficiency, which is the fraction of the proton beam passes through the entire length of the Be target and estimated using beam position monitors, must be greater than $95 \%$.

GPS Time Difference: The difference between the beam and SciBooNE detector GPS time stamps, $\left|t_{\text {beam }}-t_{\text {det }}\right|$, is required to be within $10 \mathrm{msec}$ for beam and detector information to be correctly merged.

Table 5.2 summarizes beam quality cuts and fractions of the total number of protons on target that fail each cut. The total efficiency of these cuts are 99.5\%. Pedestals, the supplied high-voltages, and responses to cosmic-ray muons of each sub-detector, and the response of SciBar to the LED light, are monitored continuously, and only the periods where all the sub-detectors are functioning are selected.

\subsubsection{SciBooNE Data Efficiency and Stability}

SciBooNE data collection during its entire run period was efficient and stable. The data collection efficiency, defined as the total data analyzed relative to the total data delivered, during its entire run period is $95.5 \%$ as shown in Fig. 5.1. Fig. 5.2 shows the constant rate for which charged current candidate events are gathered in SciBar and MRD as a function of time during the SciBooNE run period. 


\begin{tabular}{l|l|l}
\hline Cut & Parameters of Cut & Fail Fraction \\
\hline Proton Beam Intensity & TOR875 $>0.1 \times 10^{12}$ protons per pulse & $0.06 \%$ \\
Toroid Agreement & $2 \times \frac{\mid \text { TOR875-TOR860 }}{(\text { TOR875+TOR860) }}<10 \%$ & $0.07 \%$ \\
Peak Horn Current & $\left|I_{\text {peak }}\right|>170 \mathrm{kA}$ & $0.09 \%$ \\
Targeting Efficiency & $\epsilon_{\text {target }}>95 \%$ & $0.15 \%$ \\
GPS Time Difference & $\left|t_{\text {beam }}-t_{\text {det }}\right|<10 \mathrm{msec}$ & $0.13 \%$ \\
\hline
\end{tabular}

Table 5.2: SciBooNE beam quality checks and fractions of the total number of protons on target that fail each check.

\subsubsection{MiniBooNE Data Quality Checks}

MiniBooNE data must also pass five quality cuts: GPS Time Difference, Toroid Agreement, Horn Current Agreement, Targeting Efficiency, Buffer Latency. The efficiency of the MiniBooNE data quality checks is $98 \%$.

GPS Time Difference: Both the beam and MiniBooNE detector data streams must exist and have matching GPS time stamps.

Toroid Agreement: To ensure the proton beam is properly transported, the fractional difference between two torodial current transformers, TOR860 and TOR875, located at two different positions along the beamline must be within $10 \%$.

Horn Current Agreement: For accurate magnetic field focusing of the charged particles in the beamline, the absolute value of the peak magnetic horn current must be between $170 \mathrm{kA}$ and $180 \mathrm{kA}$.

Targeting Efficiency: The targeting efficiency, which is the fraction of the proton beam passes through the entire length of the Be target and estimated using beam position monitors, must be greater than $95 \%$.

\subsubsection{MiniBooNE Data Stability}

MiniBooNE data collection during its entire run period was efficient and stable. Fig. 5.3 shows the stability in the rate of neutrino and antineutrino events collected during the length of the MiniBooNE run period. 


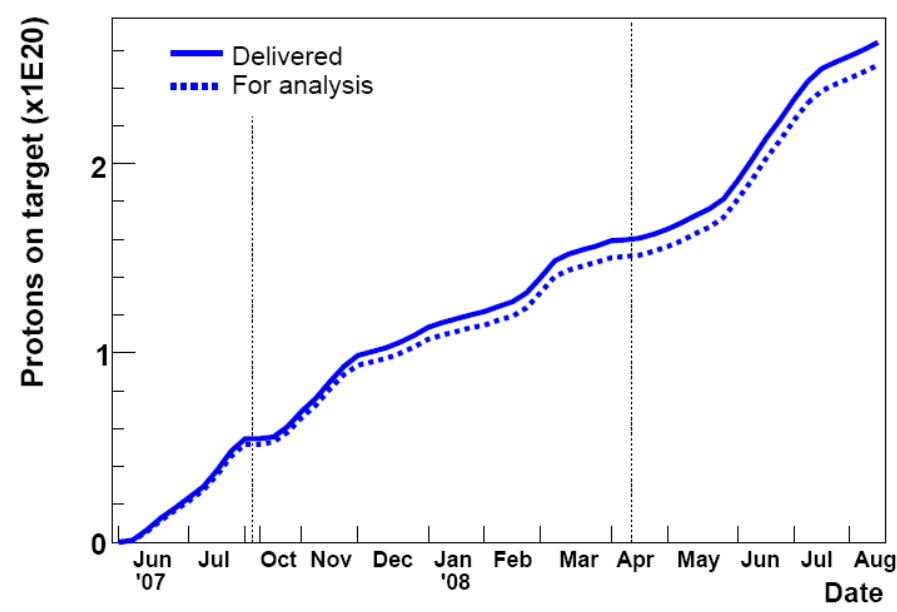

Figure 5.1: History of the accumulated number of protons on target (POT) for the entire SciBooNE data run. The first and third time periods correspond to the antineutrino mode runs used in the analysis. The solid blue line is the cumulative protons on target delivered and the dashed blue line is the cumulative protons on target collected for analysis.

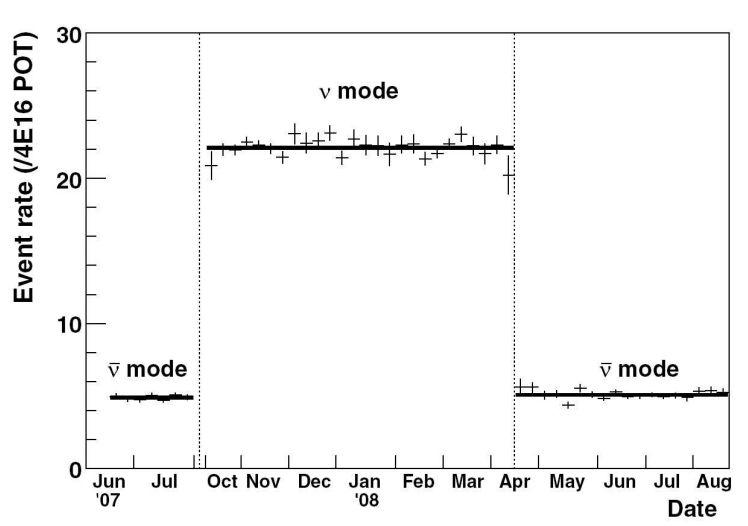

(a) SciBar Event Stability

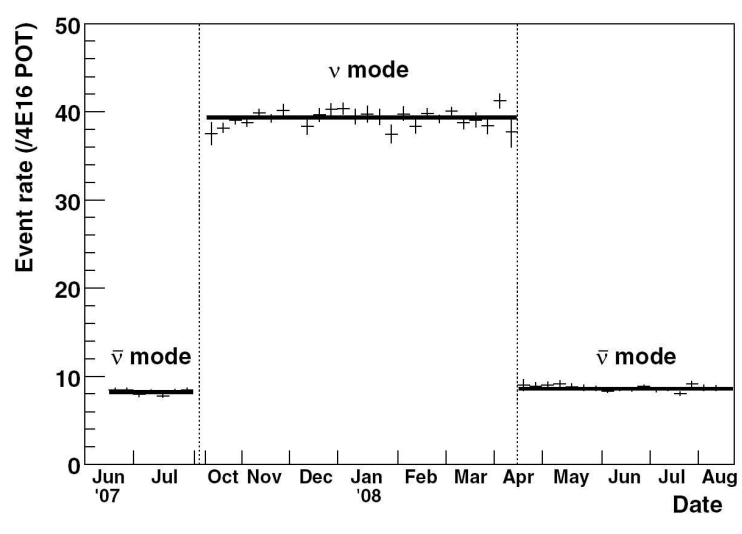

(b) MRD Event Stability

Figure 5.2: The number of charged current candidate events normalized to the number of protons on target as a function of time in SciBar (left) and in MRD (right). The first and third time periods for both figures correspond to the antineutrino mode running. 


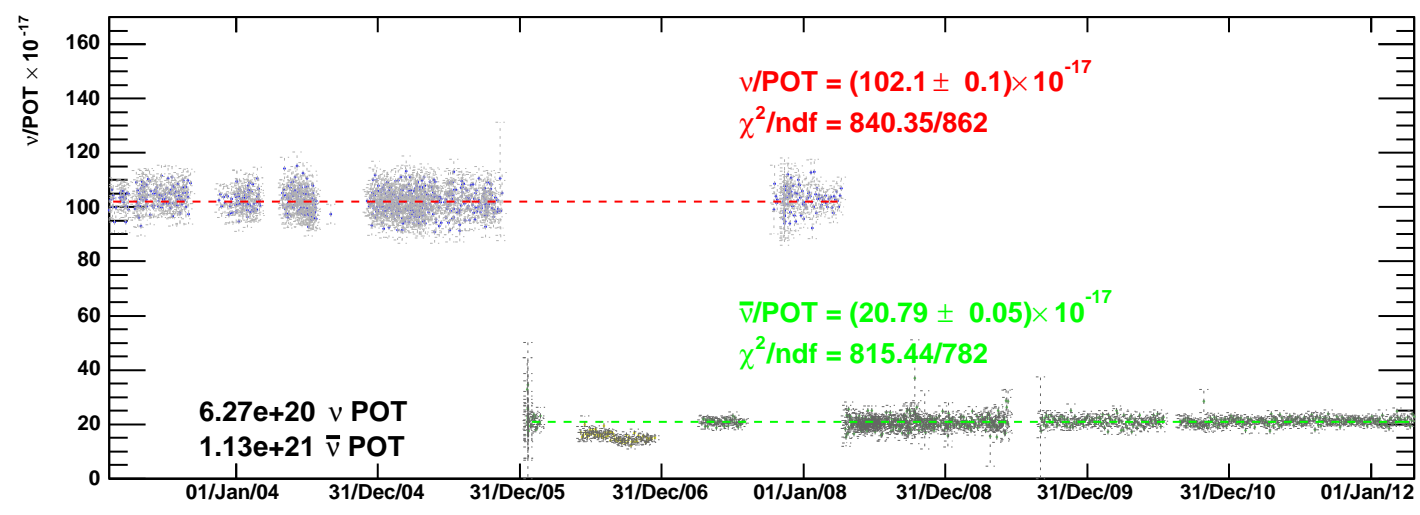

Figure 5.3: The rate at which neutrinos and antineutrino events are collected during the length of the MiniBooNE run period.

\subsection{SciBooNE CC Inclusive Event Selection}

The goal is to select muon antineutrinos that interact in the SciBar fiducial volume, resulting in a $\mu^{+}$that is fully contained in SciBar and MRD for accurate energy reconstruction. The corresponding observed event in the SciBooNE detector for this interaction will be an event containing a $\mu^{+}$track. To boost statistics, all charged current events are selected irrespective of the number of charged particles produced in association with the $\mu^{+}$. The SciBooNE detector cannot distinguish the charge of the muon $\left(\mathrm{a} \mu^{+}\right.$track in the detector is identical to a $\mu^{-}$track in the detector) so $\mu^{-}$events created from $\nu_{\mu}$ contamination in the antineutrino beam remains an intrinsic background. Figure 5.4 shows a candidate muon antineutrino event from SciBooNE data for top (X-Z plane) and side (Y-Z plane) views.

\subsubsection{Initial Cuts}

An initial set of cuts is used to select events with a candidate $\mu^{+}$track. The initial cuts remove the vast majority of events containing cosmic muons and neutrino-nucleus interaction events occurring outside the desired fiducial volume.

Selected events must contain reconstructed SciBar tracks created by charged particles originating in the SciBar fiducial volume (FV), defined to be $\pm 130 \mathrm{~cm}$ in both the $x$ and $y$ 


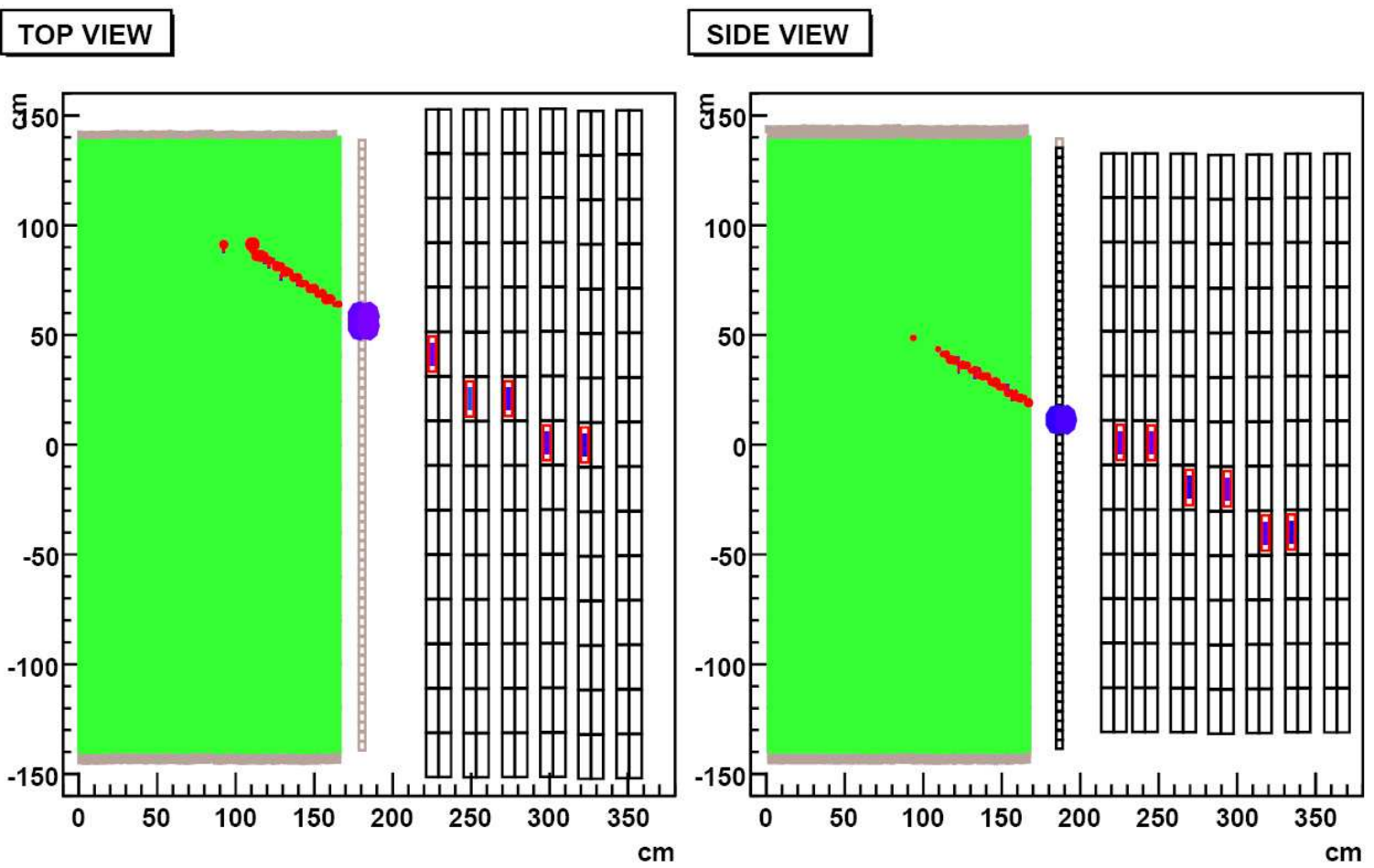

Figure 5.4: Event display in top (X-Z plane) and side (Y-Z plane) views of a muon antineutrino charged current quasielastic candidate in SciBooNE antineutrino run data. 
dimensions, and $2.62<z<157.2 \mathrm{~cm}$; this region corresponds to a total mass of 10.6 tons. This requirement removes the events with neutrino-nucleus interaction events occurring outside the SciBooNE detector.

The Muon Confidence Level (MuCL) is a particle identification variable for reconstructed SciBar tracks constructed using the observed energy deposit per SciBar layer and has the ability to distinguish different charged particles based on their $d E / d x$. More can be found in Reference 51. If there exists a SciBar track with a MuCL value above 0.05, corresponding to a high probability that a muon is selected, among all the SciBar tracks within the SciBar FV, then the event is selected and that SciBar track is designated as the candidate $\mu^{+}$track. Events with more than one SciBar track with MuCL value above 0.05 has the highest momentum track with a MuCL value above 0.05 designed as the candidate $\mu^{+}$track. In addition, the mean time of the candidate $\mu^{+}$track $(t)$ must be within the antineutrino beam time window of $0<t<2 \mu$ s to remove cosmic muons. The candidate $\mu^{+}$track must also have a reconstructed momentum (described in Subsections 5.4.2 and 5.4.3) greater than $0.25 \mathrm{GeV} / \mathrm{c}$ to reject short proton or pion tracks from NC interactions.

Events with a candidate $\mu^{+}$track are then divided into two separate sub-samples based on whether the candidate $\mu^{+}$track stops in SciBar or MRD. Selected events with the candidate $\mu^{+}$track stopping in SciBar are classified as SciBar Stopped events and selected events with the candidate $\mu^{+}$track stopping in the MRD are classified as MRD Stopped events. Both SciBar Stopped and MRD Stopped events are used in the analysis.

\subsubsection{SciBar Stopped Events}

For the event to be classified as SciBar Stopped, the candidate $\mu^{+}$track must have been fully contained within SciBar. This requires that the candidate $\mu^{+}$track for the event must not satisfy the SB-MRD matching (described in Subsection 5.1.4) with any MRD track or collection of MRD hits in the event.

All candidate $\mu^{+}$tracks are assumed to be forward going $\left(\mu^{+}\right.$with initial position upstream and final position downstream) unless there is enough information to label it as backward going ( $\mu^{+}$with initial position downstream and final position upstream). The decay $e^{+}$from the $\mu^{+}$are used to identify the direction of the candidate $\mu^{+}$tracks. The 
decay electron signal is selected by requiring delayed TDC hits at the ends of the muon tracks. The coincidence of the TDC signal from the $\mathrm{X}-\mathrm{Z}$ plane view and the $\mathrm{Y}-\mathrm{Z}$ plane view is required to remove hits from random noise or after pulses. To select the decay $e^{+}$and identify the track as backwards going, at least one delayed hit with $t>200 \mathrm{nsec}$ is required at the upstream end and no delayed hit with $t>200 \mathrm{nsec}$ is required at the downstream end. The same selection method is also used to identify the direction of $\mu^{-}$tracks created from the intrinsic $\nu_{\mu}$ background.

For SciBar Stopped events, the kinetic energy of the $\mu^{+}, E_{\mu^{+}}$, is equal to the energy of the candidate $\mu^{+}$track deposited in SciBar, $E_{\mu^{+}}^{S c i B a r} . E_{\mu^{+}}^{S c i B a r}$ is calculated from the length of the candidate $\mu^{+}$track using a range to energy conversion table shown in Figure 5.5.

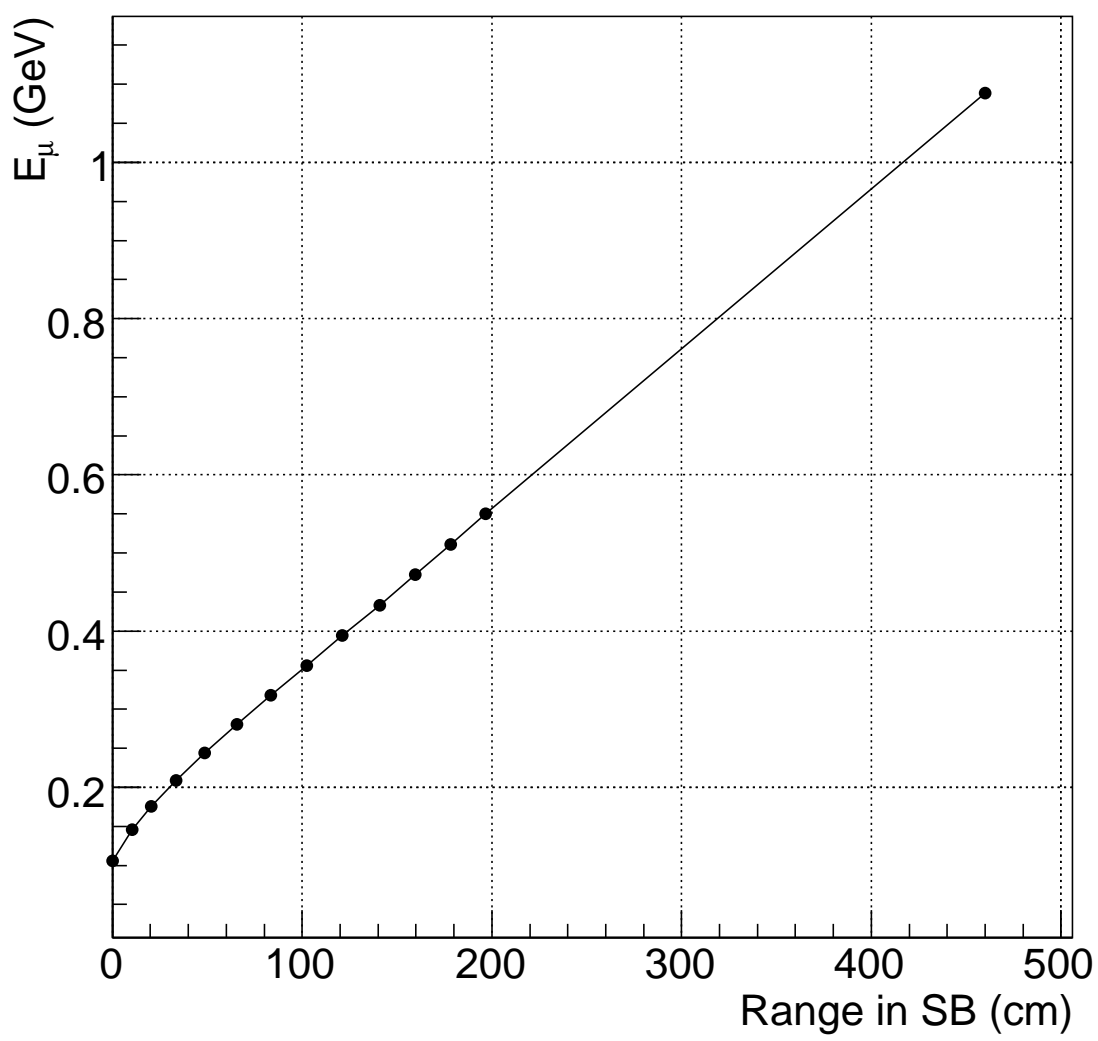

Figure 5.5: Range to muon energy conversion table used to reconstruct $E_{\mu^{+}}^{\text {SciBar }}$ for SciBar Stopped events. 
The momentum of the candidate $\mu^{+}$is calculated from the kinetic energy of the candidate $\mu^{+}$track. The angle of the candidate $\mu^{+}$is determined by the angle of the SciBar track representing the candidate $\mu^{+}$track relative to the antineutrino beam $(+z)$ direction.

Figure 5.6 shows the same SciBar Stopped event before any reconstruction and after the candidate $\mu^{+}$track has been reconstructed and selected. The green line is the reconstructed candidate $\mu^{+}$track.

\subsubsection{MRD Stopped Events}

For the event to be classified as a MRD Stopped event, the candidate $\mu^{+}$track must have been fully contained within SciBar and the MRD. This requires that the candidate $\mu^{+}$ track for the event must satisfy the SB-MRD matching (described in Subsection 5.1.4) and not exit the side or downstream end of the MRD. The latter requirement means that the downstream end of the SB-MRD matched track must be between $\pm 132 \mathrm{~cm}$ in the $x$ dimension of $\mathrm{MRD}, \pm 110 \mathrm{~cm}$ in the $y$ dimension of $\mathrm{MRD}$, and have a $z$ position upstream of the last layer of MRD.

Unlike SciBar Stopped events, all candidate $\mu^{+}$tracks for the MRD Stopped category are assumed to be forward going since the percentage of backward going candidate $\mu^{+}$tracks are estimated by simulation to be small and decay $e^{+}$tagging in the MRD is impossible.

The containment of the candidate $\mu^{+}$track within the entire SciBooNE detector ensures that the muon energy is correctly reconstructed. For MRD Stopped events, the total kinetic energy of the $\mu^{+}, E_{\mu^{+}}$, is reconstructed as:

$$
E_{\mu^{+}}=E_{\mu^{+}}^{S c i B a r}+E_{\mu^{+}}^{E C}+E_{\mu^{+}}^{W a l l}+E_{\mu^{+}}^{M R D}
$$

where $E_{\mu^{+}}^{S c i B a r}, E_{\mu^{+}}^{E C}, E_{\mu^{+}}^{W a l l}$, and $E_{\mu^{+}}^{M R D}$ are the expected kinetic energy deposit by a $\mu^{+}$in the SciBar, the EC, the wall of the dark box between EC and MRD, and the MRD, respectively. $E_{\mu^{+}}^{S c i B a r}$ for MRD Stopped events is calculated differently from $E_{\mu^{+}}^{S c i B a r}$ for SciBar Stopped events. $E_{\mu^{+}}^{S c i B a r}, E_{\mu^{+}}^{E C}$, and $E_{\mu^{+}}^{W a l l}$ are each calculated using a single length to energy conversion factor determined through MC simulation of energy deposition. The values used for the energy reconstruction are: $E_{\mu^{+}}^{S c i B a r} /($ per unit track length) $=2.04 \mathrm{MeV} / \mathrm{cm}$, $E_{\mu}^{E C}=90.8 / \cos \theta_{\mu^{+}} \mathrm{MeV}$, and $E_{\mu^{+}}^{W a l l}=3.3 / \cos \theta_{\mu} \mathrm{MeV}$, where $\cos \theta_{\mu}$ is the cosine of the 


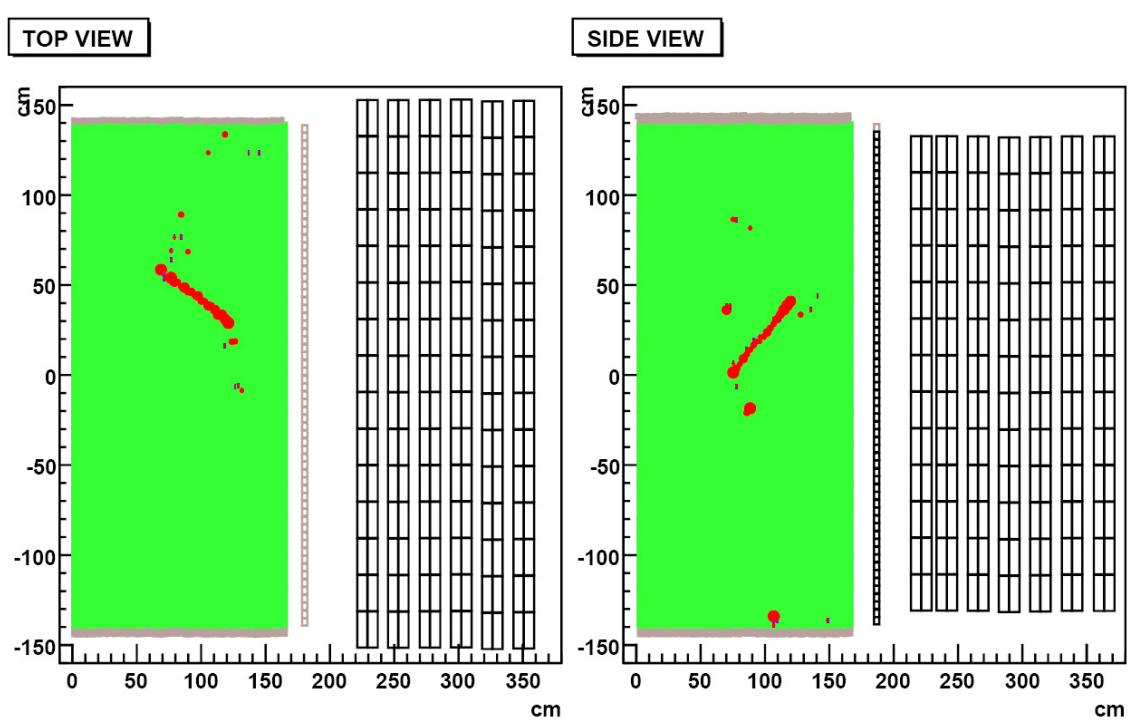

(a) Before Track Reconstruction

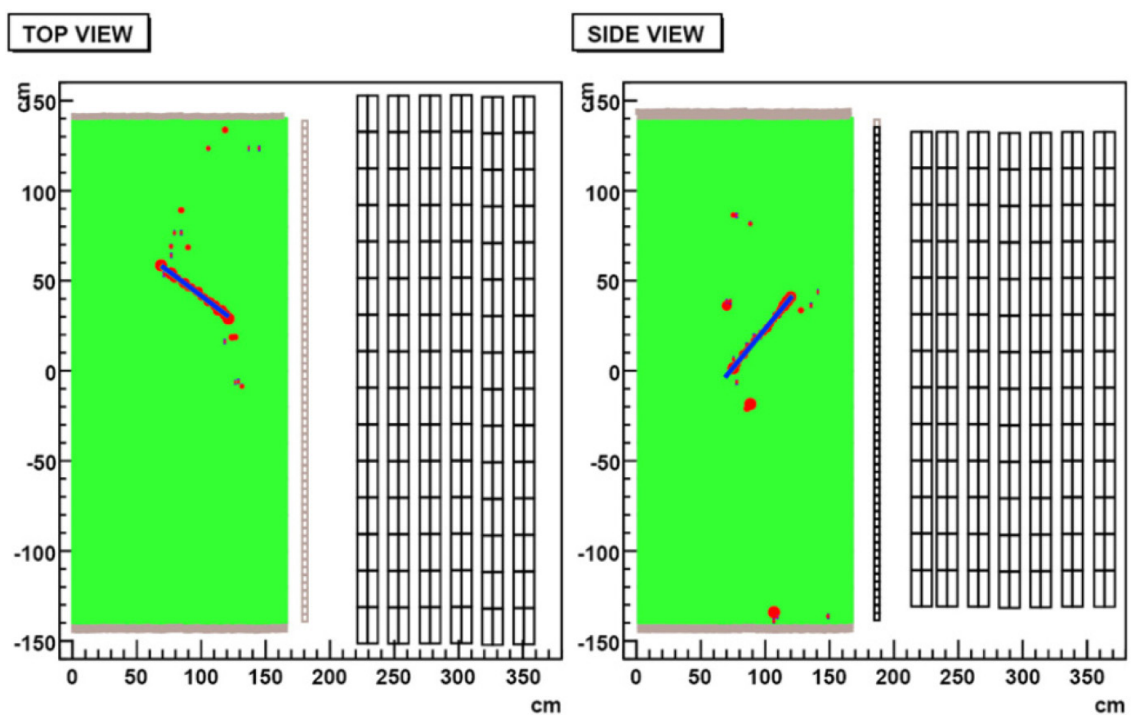

(b) After Track Reconstruction

Figure 5.6: Event display in top (X-Z plane) and side (Y-Z plane) views of a SciBar Stopped event in antineutrino run data before (top) and after (bottom) track reconstruction. The blue line is the reconstructed candidate $\mu^{+}$track.

angle of the candidate $\mu^{+}$track relative to the antineutrino beam $(+z)$ direction. $E_{\mu^{+}}^{M R D}$ is calculated using the range to energy conversion table shown in Figure 5.7. 


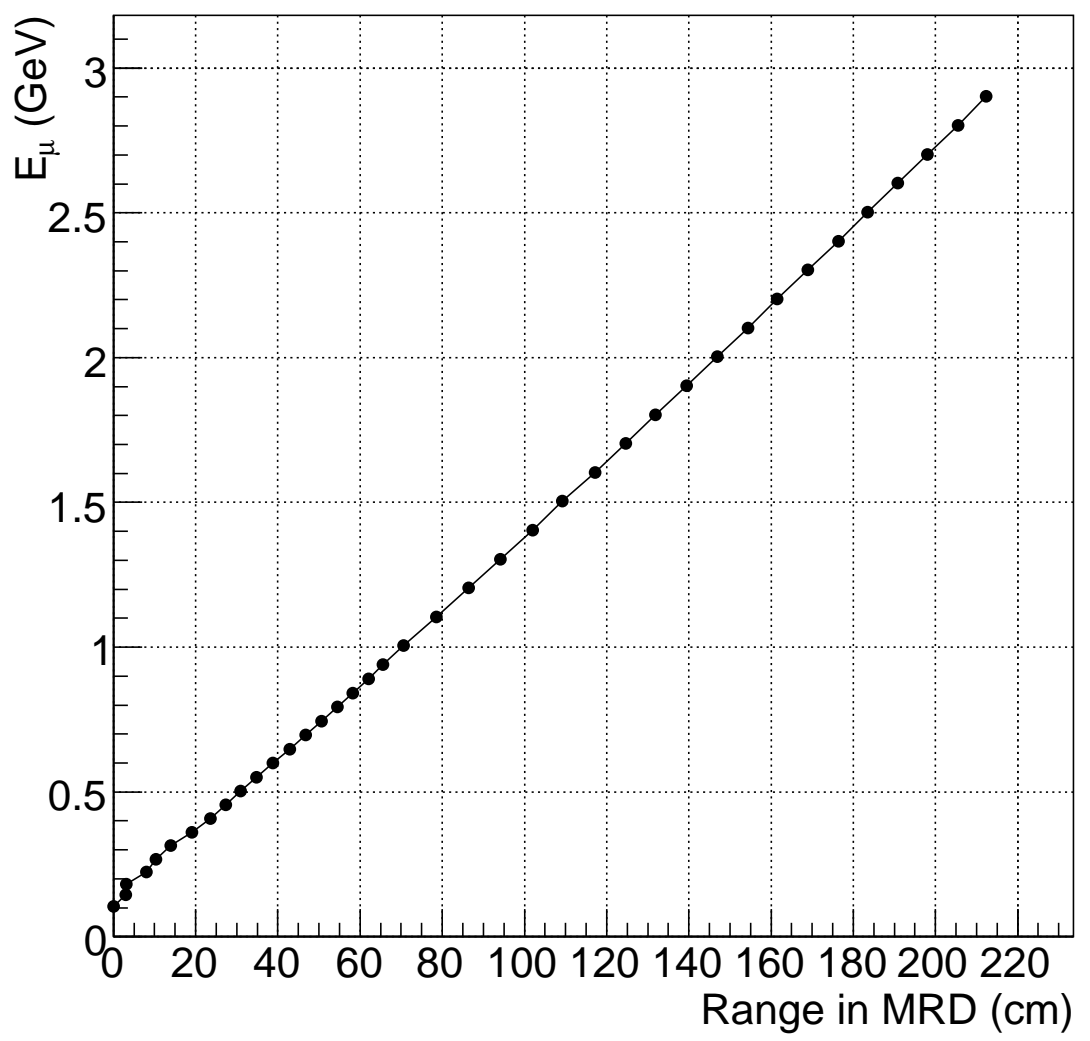

Figure 5.7: Range to muon energy conversion table used to reconstruct $E_{\mu}^{M R D}$ for MRD Stopped events.

The momentum of the candidate $\mu^{+}$is calculated from the kinetic energy of the candidate $\mu^{+}$track. The angle of the candidate $\mu^{+}$is determined by the angle of the SB-MRD Matched track representing the candidate $\mu^{+}$track relative to the antineutrino beam $(+z)$ direction.

Figure 5.8 shows the same MRD Stopped event before SB-MRD Matching and after the candidate $\mu^{+}$track has been fully reconstructed and selected.

\subsubsection{Data Events}

SciBooNE has no overburden so a small number of cosmic backgrounds do pass through the selection criteria and must be accounted for. Instead of estimating the cosmic backgrounds 


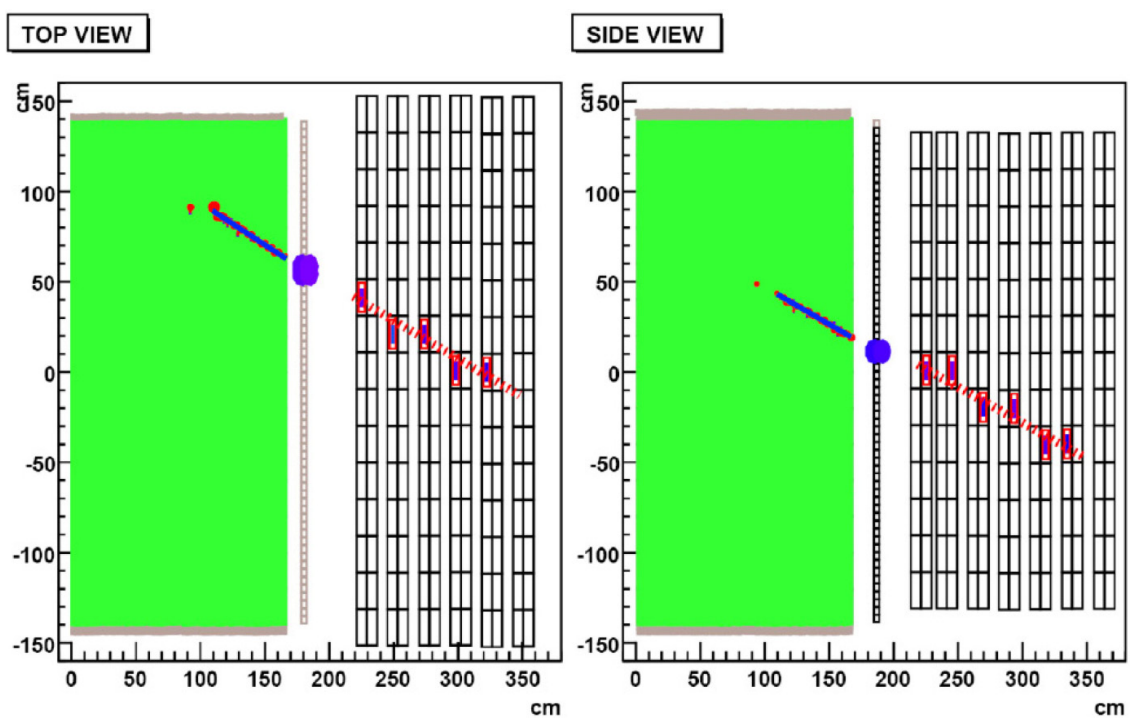

(a) Before SB-MRD Matching

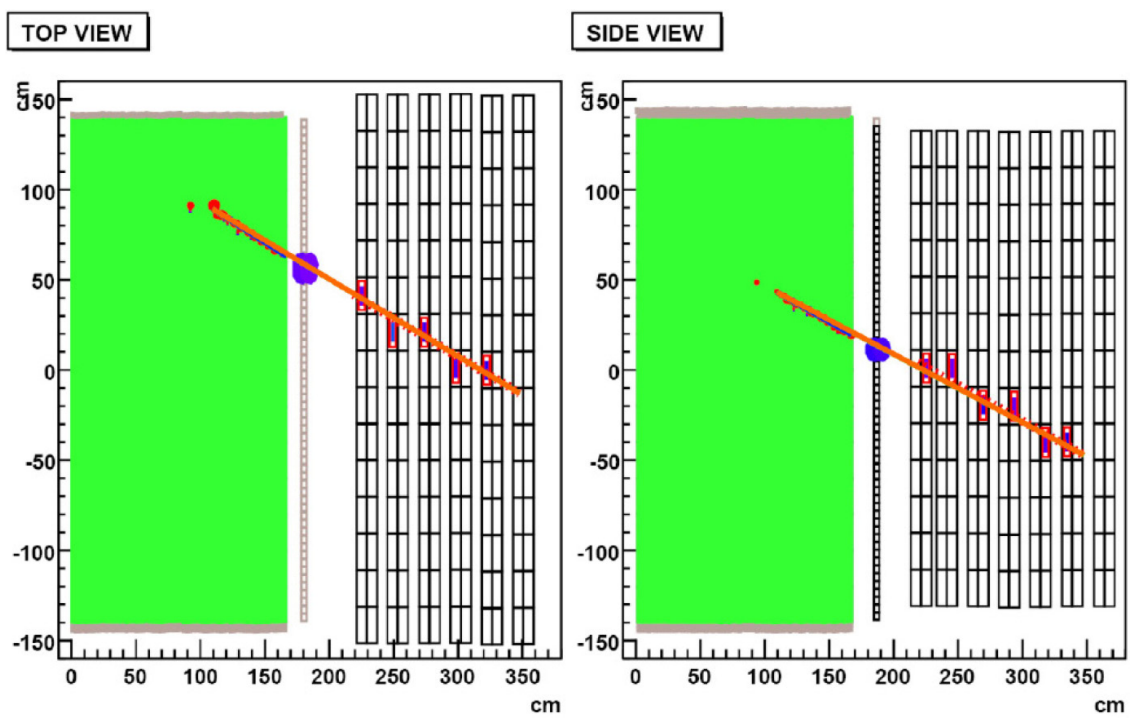

(b) After SB-MRD Matching

Figure 5.8: Event display in top (X-Z plane) and side (Y-Z plane) views of a MRD Stopped event in antineutrino run data before (top) and after (bottom) SB-MRD matching. In the top figure, the blue line is the reconstructed SciBar track and the dashed red line is the reconstructed MRD track. The long orange line in the bottom figure is the reconstructed candidate $\mu^{+}$track. 
in the data through MC simulation, cosmic backgrounds are estimated when the neutrino beam is turned off and its estimation is subtracted from data. For cosmic background estimation, the same muon selection criteria are applied to a beam-off time window that is five times longer than the beam-on window for better statistics. This event rate is scaled down by a factor of five and subtracted from the beam-on data. After selection, the beam-off data is less than $8 \%$ of the beam-on data.

\subsection{MiniBooNE CCQE Event Selection}

The goal of the selection cuts is to select events where a muon antineutrinos undergoes a quasielastic charged current interaction in the MiniBooNE fiducial volume. The interaction proceeds as follows:

$$
\begin{array}{lll}
1: & \bar{\nu}_{\mu}+p \rightarrow & \mu^{+}+n \\
2: & & \hookrightarrow e^{+}+\nu_{e}+\bar{\nu}_{\mu}
\end{array}
$$

where each line (labeled 1 and 2) represents a separate MiniBooNE subevent. The resulting signal is therefore two subevents both from the produced $\mu^{+}$, one associated with the $\mu^{+}$ itself and the other associated with its decay $e^{+}$.

One large source of background is $\bar{\nu}_{\mu}$ undergoing charged current single-pion production, which proceeds as follows:

$$
\begin{array}{ccc}
1: & \bar{\nu}_{\mu}+n \rightarrow \mu^{+}+n+\pi^{-} & \\
& \hookrightarrow \mu^{-}+\bar{\nu}_{\mu} \\
2: & \hookrightarrow e^{+}+\nu_{e}+\bar{\nu}_{\mu} \\
3: & \hookrightarrow e^{-}+\bar{\nu}_{e}+\nu_{\mu}
\end{array}
$$

where each line (labeled 1 and 2 and 3) represents a separate MiniBooNE subevent. The $\pi^{-}$decays immediately and light from the $\mu^{-}$gets added to the light from the primary antineutrino interaction in subevent 1 . Though this background interaction generally has 3 subevents, it is possible for the $\pi^{-}$in subevent 1 to be absorbed in the nucleus resulting in the lack of subevent 3 or it is possible for the $\mu^{-}$in subevent 1 to be captured on a ${ }^{12} \mathrm{C}$ resulting in the lack of subevent 3 , mimicking the desired two subevent signal. 


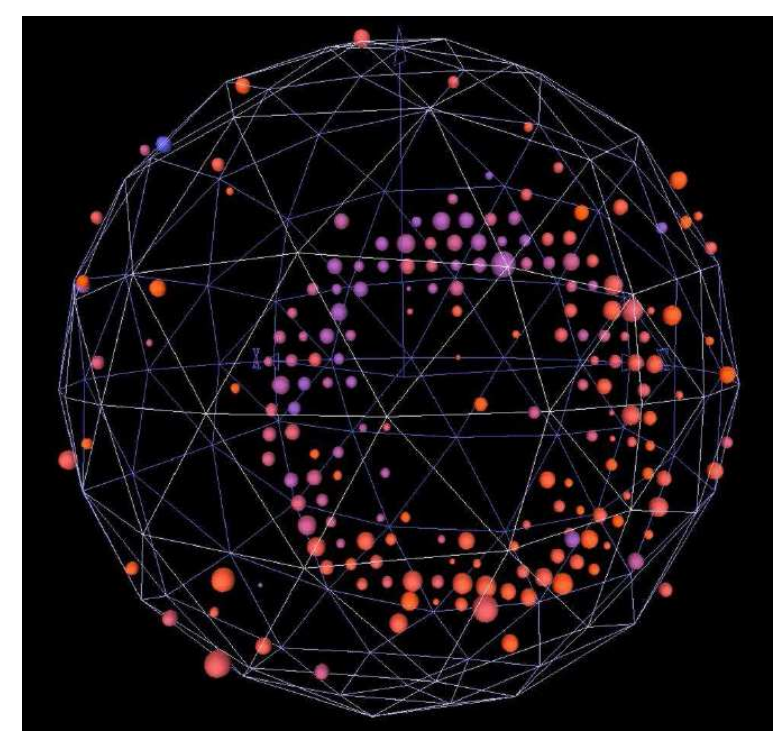

Figure 5.9: Event display of a muon antineutrino charged current quasielastic candidate in MiniBooNE.

The MiniBooNE detector cannot distinguish between a particle's charge (a $\mu^{+}$track in the detector is identical to a $\mu^{-}$track in the detector) so a $\mu^{-}$created via quasielastic charged current interaction from $\nu_{\mu}$ contamination in the antineutrino beam remains an intrinsic background. Some of the background $\mu^{-}$s are captured on carbon nuclei and this process is simulated in the MC. These specific background events are therefore not selected. Figure 5.9 shows a candidate muon antineutrino event from MiniBooNE.

The sequence of cuts used to select the MiniBooNE candidate CCQE $\mu^{+}$events are summarized in Table 5.3 .

The first cut (Cut 1), which is the number of veto hits $<6$ per event, rejects events containing cosmic muons and antineutrino-induced or neutrino-induced events occurring outside the MiniBooNE main detector volume. The first cut also removes events where any of the antineutrino or neutrino interaction products escape the main detector volume, ensuring that correct energy reconstruction, or events produced in the surrounding material. The second cut (Cut 2) requires that the candidate $\mu^{+}$is produced in coincidence with the antineutrino beam window and removes random cosmic or muon decays. The third cut (Cut 3) ensures that the reconstructed primary $\mu^{+}$vertex is located within a fiducial region 


\begin{tabular}{l|l}
\hline \hline Cut \# & Description \\
1 & all subevents, \# of veto hits $<6$ \\
2 & 1st subevent, event time window, $4400<T(\mathrm{~ns})<6400$ \\
3 & 1st subevent, reconstructed vertex radius $<500 \mathrm{~cm}$ \\
4 & 1st subevent, kinetic energy $>200 \mathrm{MeV}$ \\
5 & 1st subevent, $\mu / e$ log-likelihood ratio $>0.0$ \\
6 & \# total subevents $=2$ \\
7 & 1st subevent, minimum $\mu-e$ vertex separation distance \\
\hline \hline
\end{tabular}

Table 5.3: List of cuts for the MiniBooNE CCQE $\mu^{+}$event selection.

in the main detector volume sufficiently far from the PMTs for accurate reconstruction. The fourth cut (Cut 4) sets a minimum lower bound on the candidate $\mu^{+}$kinetic energy for reliable reconstruction.

The fifth cut (Cut 5) requires that the candidate primary $\mu^{+}$is better fit as a $\mu^{ \pm}$than as a $e^{ \pm}$. Misreconstructed and multi-particle events tend to prefer the $e^{ \pm}$hypothesis so this cut reduces these kind of background events. More information about the $\mu / e$ log-likelihood ratio can be found in Reference 99]. The sixth cut (Cut 6) extracts the CCQE antineutrino interaction by requiring exactly 2 subevents. At least 20 PMT hits are required for the latter subevent to reduce the probability of accidental coincidences with the initial antineutrino interaction.

The seventh cut (Cut 7) uses the $\mu$-e vertex separation distance, the measured separation between the reconstructed $\mu$ and $e$ vertices, to ensure that the $e^{+}$is correctly associated with the $\mu^{+}$. The value of the minimum $\mu-e$ vertex separation distance must be at least $100 \mathrm{~cm}$ and increases further as a function of $\mu^{+}$kinetic energy. More information about the cut can be found in Reference [75].

After all cuts, the candidate $\mu^{+}$kinematics are determined through the MiniBooNE event reconstruction model described in Section 5.2 . 


\subsection{Antineutrino Energy Reconstruction}

The $\bar{\nu}_{\mu}$ energy can be entirely reconstructed using the kinematics of the detected candidate $\mu^{+}$along with a few reasonable assumptions. The $\bar{\nu}_{\mu}$ energy in both the selected MiniBooNE CCQE interaction and SciBooNE CC interaction events are reconstructed in exactly the same method.

Though the selected events include $\bar{\nu}_{\mu}$ and $\nu_{\mu}$ interactions on carbon and hydrogen in the detectors, the reconstructed antineutrino energy is based on the assumption that the interaction is always a $\bar{\nu}_{\mu}$ CCQE interaction with a proton at rest in carbon: $\bar{\nu}_{\mu}+p \rightarrow \mu^{+}+n$. Hence, it is just a function of the measured energy and direction of the outgoing $\mu^{+}$. The equation for reconstructed antineutrino energy is:

$$
E_{\nu}^{Q E}=\frac{M_{n}^{2}-\left(M_{p}-E_{B}\right)^{2}-M_{\mu}^{2}+2\left(M_{p}-E_{B}\right) E_{\mu}}{2\left(M_{p}-E_{B}-E_{\mu}+P_{\mu} \cos \theta_{\mu}\right)},
$$

where $M_{n}$ and $M_{p}$ are the mass of the neutron and proton, $M_{\mu}, E_{\mu}, P_{\mu}$, and $\theta_{\mu}$ are the mass, total energy, momentum, and direction of the outgoing $\mu^{+}$, and $E_{B}$ is the binding energy (30 MeV for protons in carbon). The total energy $E_{\mu}$ of the outgoing $\mu^{+}$is just the sum of the total kinetic energy $E_{\mu^{+}}$of the $\mu^{+}$(the value extracted from the MiniBooNE event reconstruction single track model described in Section 5.2 for MiniBooNE events and defined in Subsections 5.4.2 and 5.4.3 for SciBar Stopped and MRD Stopped events, respectively) and the rest mass of the $\mu^{+}$.

Equation 5.2 is applied to all selected MiniBooNE and SciBooNE events in data and $\mathrm{MC}$, even though a sizeable fraction of the events are from the $\nu_{\mu}$ in the antineutrino beam and/or are not CCQE (i.e. charged current single $\pi(\mathrm{CC} 1 \pi)$, charged current multi-pion (CC multi-pion), or NC events misidentified as CCQE events). The impact of the CCQE reconstruction assumption, which leads to reduced accuracy in reconstructed energy for non-CCQE events, is accounted for in $\mathrm{MC}$, which includes these selected non-CCQE events and various effects associated with scattering off the carbon nucleus.

Figure 5.10 show the reconstructed antineutrino energy resolution, defined as:

$$
\frac{\text { reconstructed } E_{\nu}^{Q E}-\text { true } E_{\nu}}{\operatorname{true} E_{\nu}}
$$


for all MC selected events and true CCQE MC selected events in SciBooNE. Since SciBoooNE selects for all CC events, selected events with associated $\pi$ production underestimates the reconstructed antineutrino energy because the $\pi$ carries away energy and the antineutrino energy is solely reconstructed from the muon energy. Hence, these events create the antineutrino energy resolution hump at values less than 0 in (a) of Figure 5.10 . Table 5.4 shows the standard deviations of the Gaussian fits to the reconstructed antineutrino energy resolution distributions for selected MC MiniBooNE and SciBooNE CCQE and total events. Figure 5.11 shows the reconstructed $E_{\nu}^{Q E}$ compared to the true $E_{\nu}$ for selected MC CCQE events and non-CCQE events in MiniBooNE.

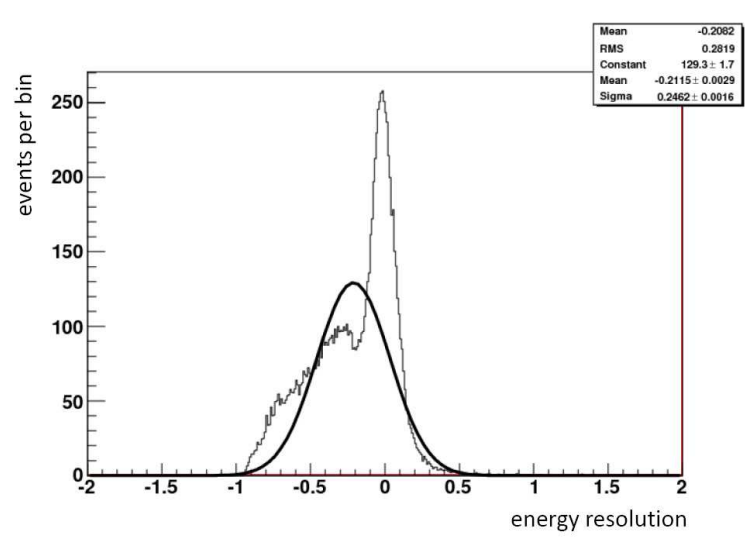

(a) SciBooNE $E_{\nu}$ Resolution for all MC Events

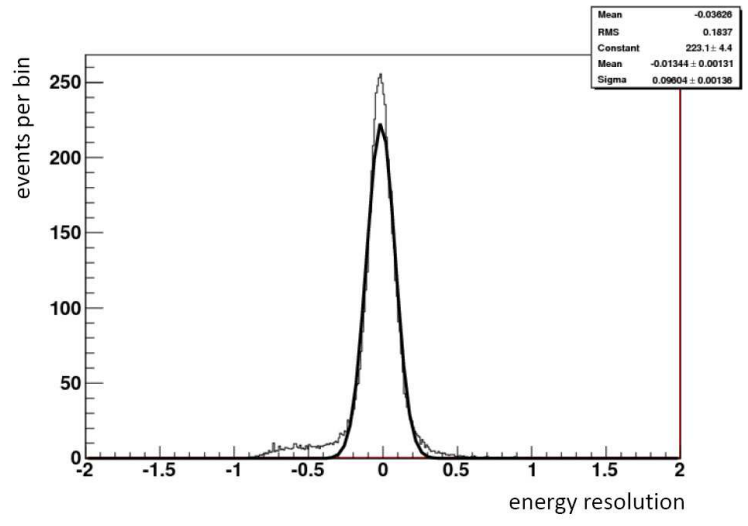

(b) SciBooNE $E_{\nu}$ Resolution for true CCQE MC Events

Figure 5.10: SciBooNE reconstructed antineutrino energy resolution ( $\frac{\text { reconstructed } E_{\nu}^{Q E}-\text { true } E_{\nu}}{\text { true } E_{\nu}}$ ) for all and true CCQE selected MC events. The black curves show the Gaussian fits to the reconstructed antineutrino energy resolution.

The reconstructed antineutrino energy, $E_{\nu}^{Q E}$, for selected MiniBooNE and SciBooNE data and $\mathrm{MC}$ events are binned in a 21-bin histogram that captures the shape of the energy distribution while maintaining adequate statistics in each bin. The binning goes from 300 $\mathrm{MeV}$ to $1.9 \mathrm{GeV}$, with individual bin widths as follows: bin 1, $100 \mathrm{MeV}$; bins 2-19, 66.7 $\mathrm{MeV}$; bin 20, $100 \mathrm{MeV}$; bin 21, $200 \mathrm{MeV}$. The first and last two bins are wider to ensure adequate event statistics in data and MC. 


\begin{tabular}{l|c|c}
\hline \hline interaction type & MiniBooNE & SciBooNE \\
\hline CCQE & $8.3 \%$ & $9.6 \%$ \\
\hline total & $13.9 \%$ & $24.6 \%$ \\
\hline \hline
\end{tabular}

Table 5.4: The standard deviations $(\sigma)$ of the Gaussian fits to the reconstructed antineutrino energy resolution distributions for selected MC MiniBooNE and SciBooNE CCQE and total events.

\subsection{Composition of the Selected MiniBooNE and SciBooNE Events}

The composition and properties of the selected MiniBooNE and SciBooNE events is understood by examining the truth variables of the selected MC simulation events. Figure 5.12 shows the predictions for reconstructed antineutrino and neutrino energy distributions, which corresponds to the selected $\bar{\nu}_{\mu}$ and $\nu_{\mu}$ events in the antineutrino beam, in MiniBooNE by interaction type: CCQE, CC1 $\pi$, and all other interaction types (CC multi-pion and NC). Figure 5.13 shows the predictions for reconstructed antineutrino and neutrino energy distribution in SciBooNE by interaction type: CCQE, CC1 $\pi$, and other (CC multi-pion and NC). $\nu_{e}$ and $\bar{\nu}_{e}$ events in the selected MiniBooNE and SciBooNE events are negligible and not shown.

Table 5.5 shows the numerical values for the MC predictions of selected MiniBooNE and SciBooNE events by neutrino and interaction type. The efficiency, defined as the number of selected MC events of a specific type divided by the number of MC events of the same type predicted to interact in the fiducial volume of the detector, of the MiniBooNE selection criteria for CCQE $\bar{\nu}_{\mu}$ events is $30 \%$. The efficiencies of the SciBooNE selection criteria for CC inclusive and CCQE $\bar{\nu}$ events are $37 \%$ and $35 \%$, respectively.

Figure 5.14 shows the predicted reconstructed antineutrino and neutrino energy distributions for selected events on hydrogen and carbon nuclei in MiniBooNE. Figure 5.15 shows the predicted reconstructed antineutrino and neutrino energy distributions for selected events on hydrogen and carbon nuclei in SciBooNE. 


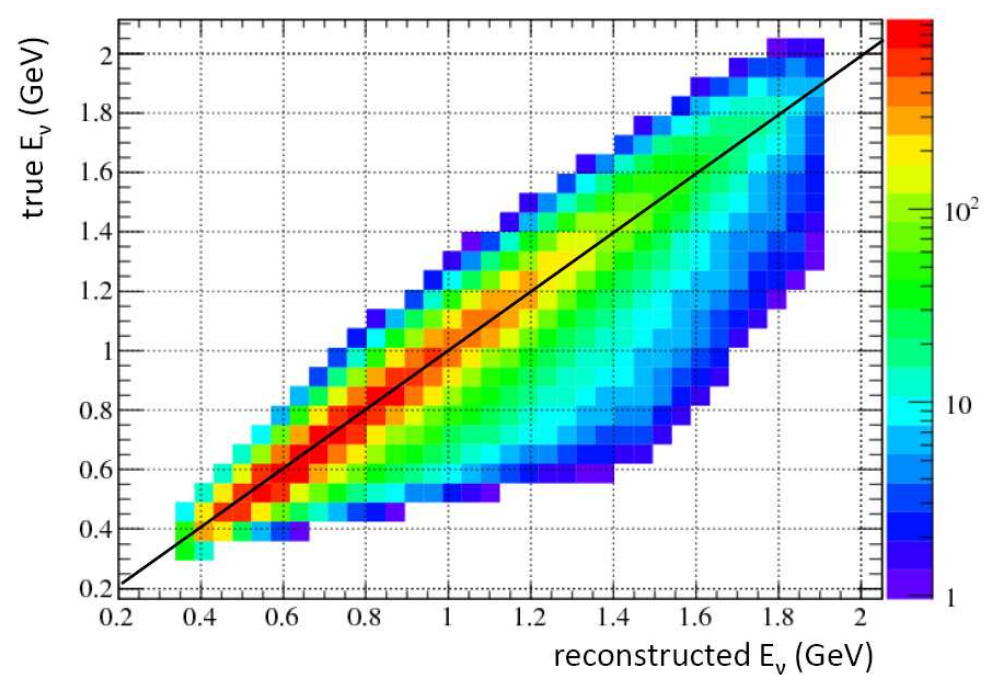

(a) CCQE events

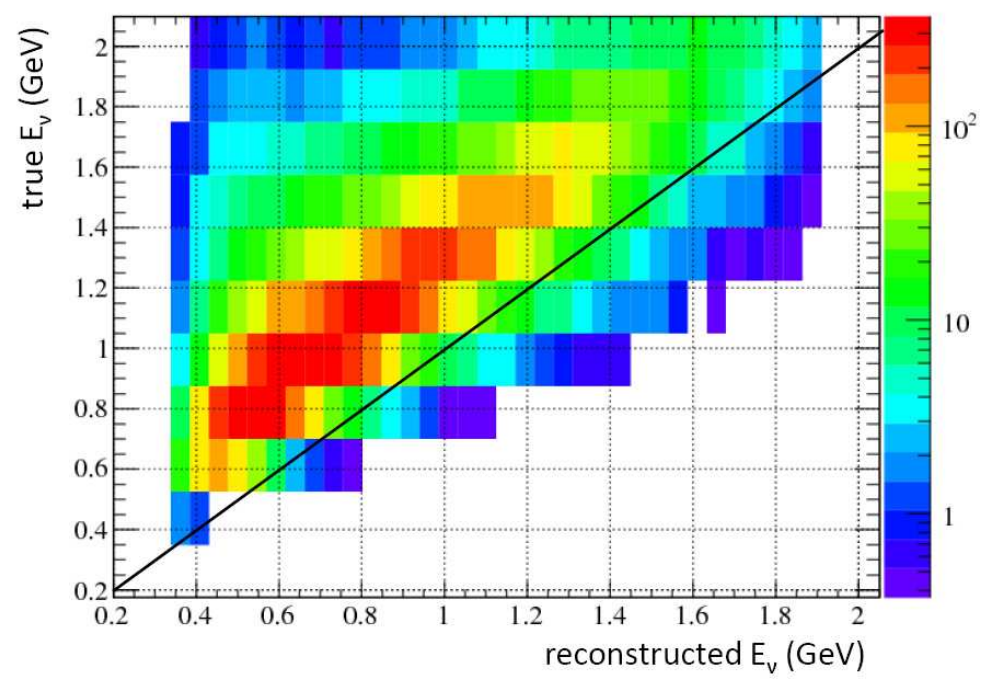

(b) non-CCQE events

Figure 5.11: 2D histogram of true $E_{\nu}$ versus reconstructed $E_{\nu}^{Q E}$ for selected CCQE events (top) and selected non-CCQE events in MiniBooNE. The black line is at $\mathrm{y}=\mathrm{x}$. 


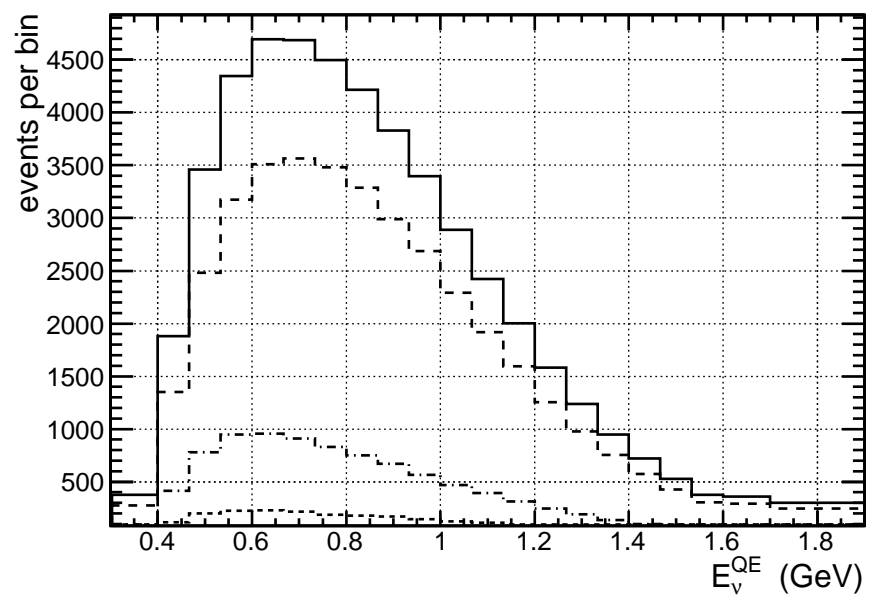

(a) MiniBooNE RS Events

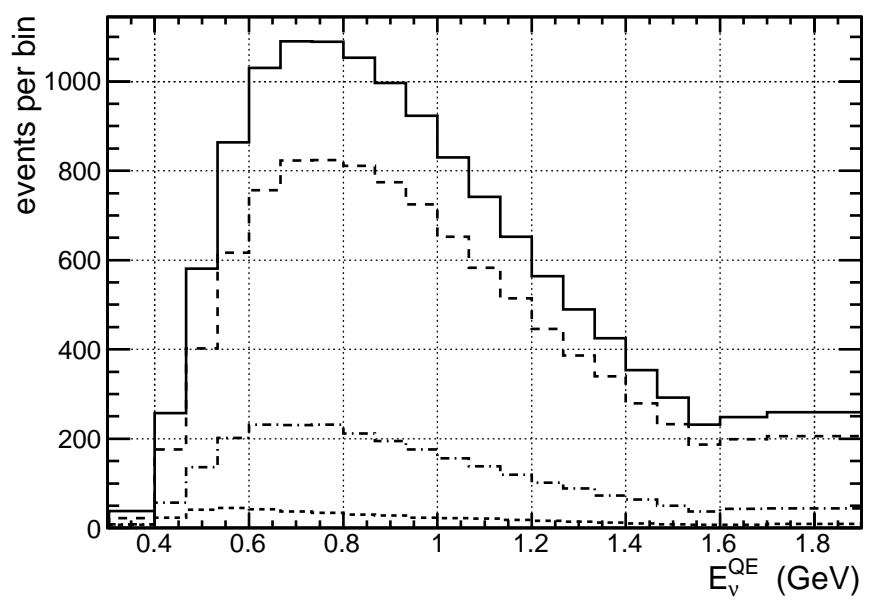

(b) MiniBooNE WS Events

Figure 5.12: Reconstructed antineutrino and neutrino energy $\left(E_{\nu}^{Q E}\right)$ distributions for selected RS and WS MiniBooNE events for different interaction types (CCQE, CC1 $\pi$, other) from MiniBooNE MC. Total events are represented by the solid line, CCQE interaction events are represented by the dashed line, $\mathrm{CC} 1 \pi$ interaction events are represented by the dot-dashed line, and all other interaction (CC multi- $\pi$ or NC) events are represented by the short-dashed line. 


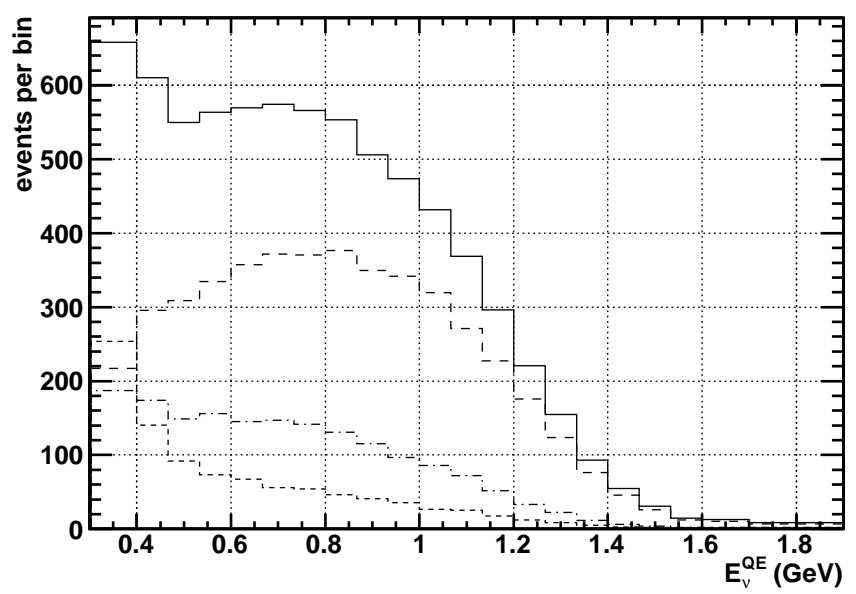

(a) SciBooNE RS Events

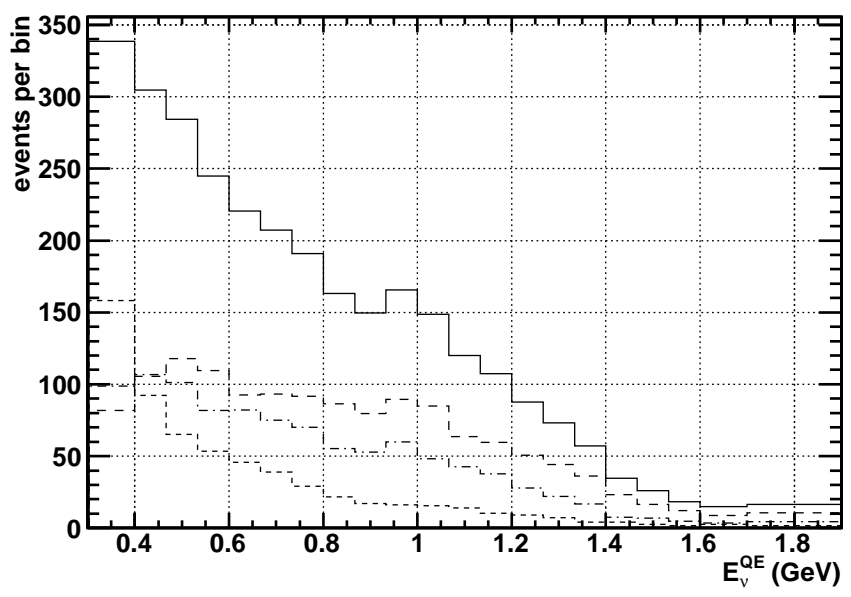

(b) SciBooNE WS Events

Figure 5.13: Reconstructed antineutrino and neutrino energy $\left(E_{\nu}^{Q E}\right)$ distributions for selected RS and WS SciBooNE events for different interaction types (CCQE, CC1 $\pi$, other) from SciBooNE MC. Total events are represented by the solid line, CCQE interaction events are represented by the dashed line, $\mathrm{CC} 1 \pi$ interaction events are represented by the dot-dashed line, and all other interaction (CC multi- $\pi$ or NC) events are represented by the short-dashed line. 


\begin{tabular}{l|c|c|c|c}
\hline \hline & \multicolumn{2}{|c|}{ MiniBooNE } & \multicolumn{2}{c}{ SciBooNE } \\
\hline interaction type & $\bar{\nu}$ events & $\nu$ events & $\bar{\nu}$ events & $\nu$ events \\
\hline CCQE & 37428 & 9955 & 4619 & 1359 \\
\hline $\mathrm{CC} 1 \pi$ & 8961 & 2593 & 1735 & 1006 \\
\hline $\mathrm{CC}$ multi- $\pi$ or NC & 2364 & 460 & 959 & 610 \\
\hline Total & 48753 & 13008 & 7313 & 2975 \\
\hline \hline
\end{tabular}

Table 5.5: MC predictions for the number of selected events by neutrino and interaction type for both MiniBooNE and SciBooNE.

The difference in shape between the SciBooNE RS (WS) and MiniBooNE RS (WS) energy distributions is mainly due to different event selection criteria between MiniBooNE and SciBooNE. MiniBooNE selects for CCQE interaction events and SciBooNE selects for all CC interaction events so the SciBooNE sample has a larger percentage of nonCCQE interaction events. Since the antineutrino energy reconstruction is based on a CCQE interaction assumption, there are more SciBooNE events with a larger discrepancy between true antineutrino energy and reconstructed antineutrino energy than in MiniBooNE, leading to shape differences. Differences in selection efficiency, antineutrino flux at the detector locations, and background rejection between MiniBooNE and SciBooNE also contribute to the shape differences.

Figure 5.16 shows the distribution of the combined antineutrino and neutrino propagation distances, from production in the decay tunnel to interaction in SciBooNE or MiniBooNE. The difference in the MiniBooNE and SciBooNE distribution shapes is due to a combination of different selection efficiencies and path length resolutions between MiniBooNE and SciBooNE. MiniBooNE selects proportionally more neutrino and antineutrino events from lower energy $\pi$ and $K$ than SciBooNE and lower energy $\pi$ and $K$ tend to decay upstream in the decay tunnel, leading to a longer path length. In addition, SciBooNE is much closer to the neutrino and antineutrino production source than MiniBooNE so SciBooNE has better path length resolution than MiniBooNE (MiniBooNE path length resolution is smeared over the much longer distance). Since MiniBooNE and SciBooNE both 


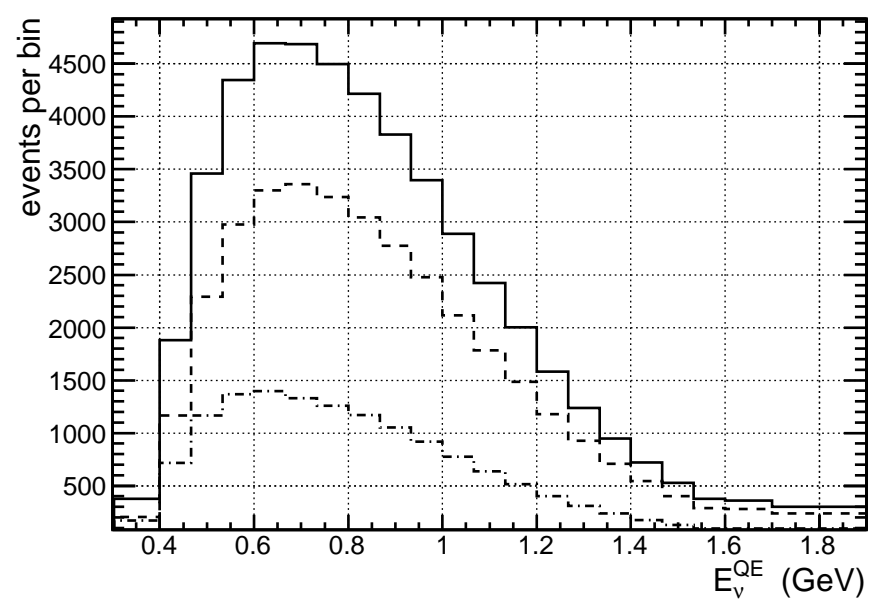

(a) MiniBooNE RS Events

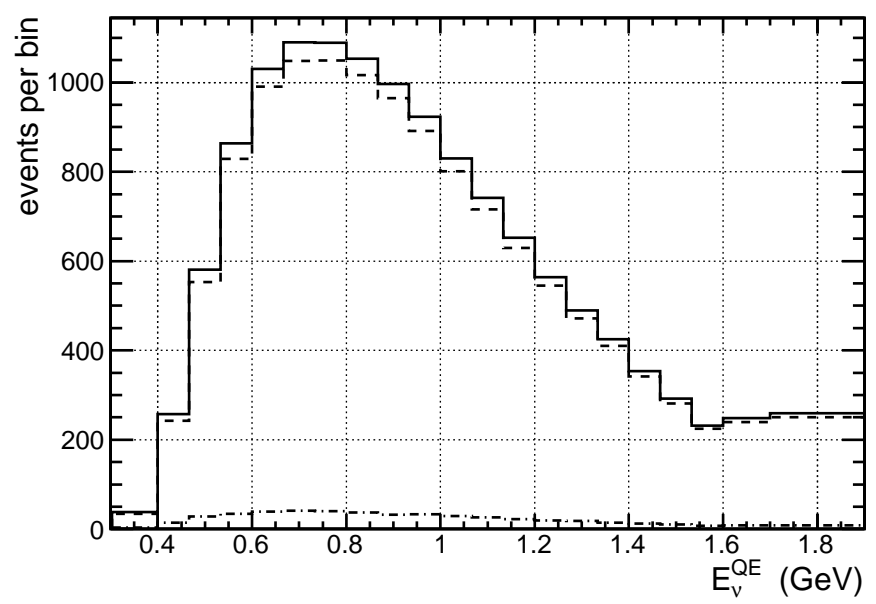

(b) MiniBooNE WS Events

Figure 5.14: Reconstructed antineutrino and neutrino energy $\left(E_{\nu}^{Q E}\right)$ distributions for selected RS and WS MiniBooNE events on different target types (hydrogen or carbon) from MiniBooNE MC. Total events are represented by the solid line, events with interaction on carbon are represented by the dashed line, and events with interaction on hydrogen are represented by the dot-dashed line. 


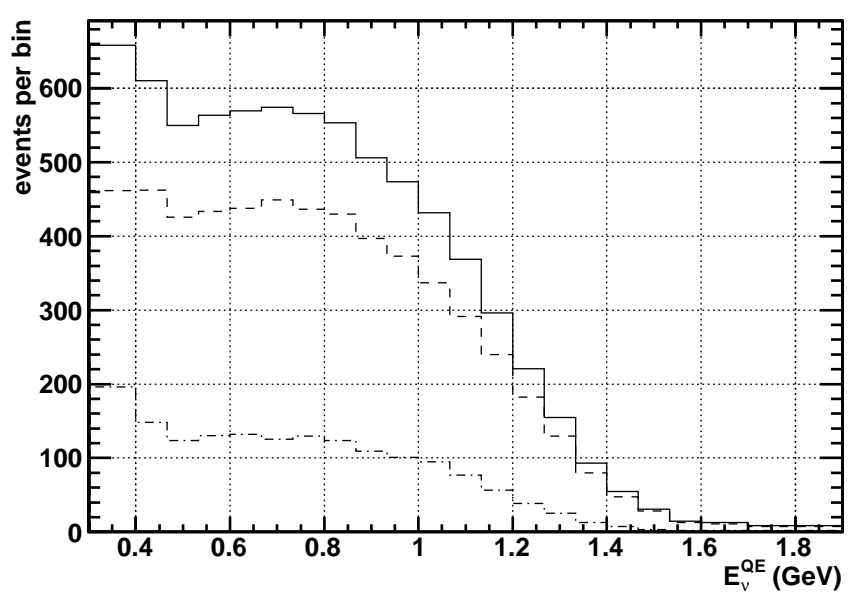

(a) SciBooNE RS Events

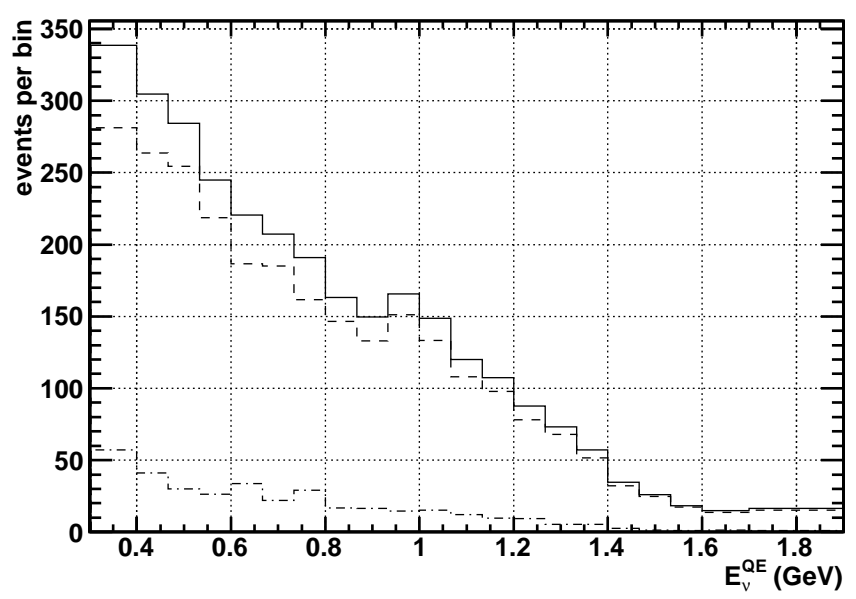

(b) SciBooNE WS Events

Figure 5.15: Reconstructed antineutrino and neutrino energy $\left(E_{\nu}^{Q E}\right)$ distributions for selected RS and WS SciBooNE events on different target types (hydrogen or carbon) from SciBooNE MC. Total events are represented by the solid line, events with interaction on carbon are represented by the dashed line, and events with interaction on hydrogen are represented by the dot-dashed line. 
select low energy neutrinos from $\pi^{+}$and $K^{+}$produced at the upstream end of the decay tunnel (with the longer neutrino path length), these low energy neutrino events manifest as a sharp peak at large path length $(\approx 100 \mathrm{~m})$ in SciBooNE while these low energy events are smeared out over the longer path lengths in MiniBooNE.

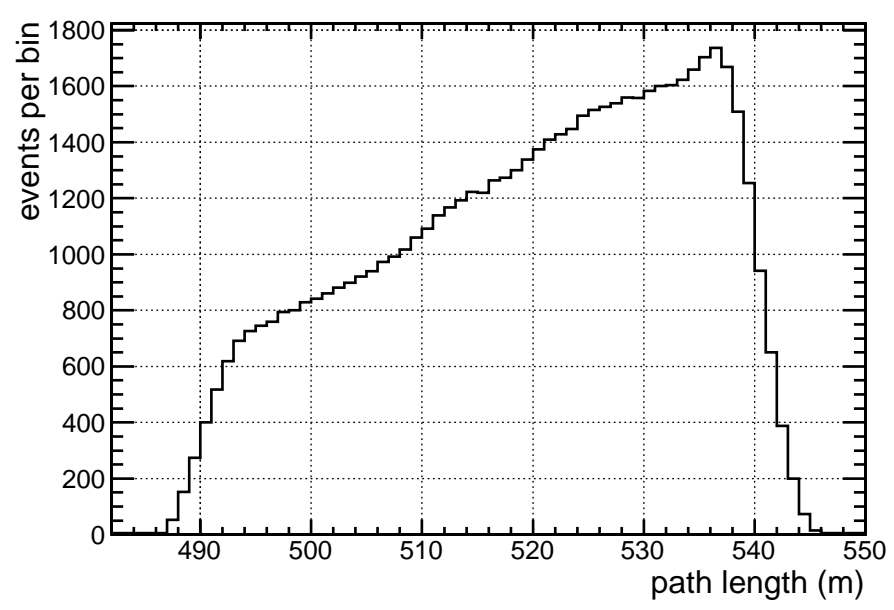

(a) MiniBooNE (anti)neutrino path lengths

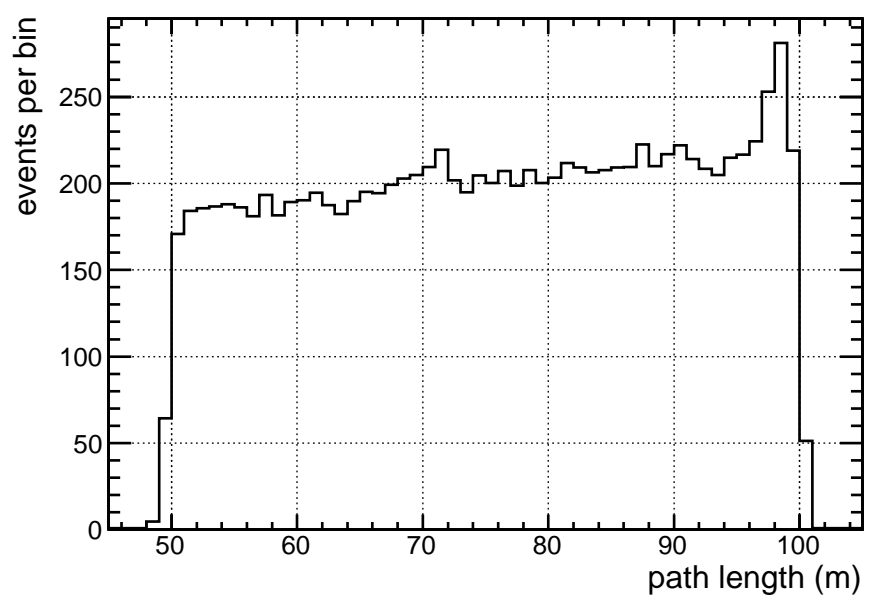

(b) SciBooNE (anti)neutrino path lengths

Figure 5.16: Antineutrino and neutrino path lengths for MiniBooNE and SciBooNE events from point of production to interaction in detector, as predicted by the MC simulation. 


\subsection{Event Reconstruction and Selection Results}

In conclusion, the combination of accurate event reconstruction and selection effectively produces significant samples with large percentages of the desired MiniBooNE CCQE and SciBooNE CC inclusive events. Though $\nu$ events are an intrinsic background and cannot be removed through selection, $\mathrm{MC}$ predicts that $61 \%$ of all events selected in MiniBooNE are CCQE $\bar{\nu}$ events and $71 \%(45 \%)$ of all events selected in SciBooNE are CC inclusive $(\mathrm{CCQE}) \bar{\nu}$ events. The MiniBooNE selection criteria retains $30 \%$ of all CCQE $\bar{\nu}_{\mu}$ events interacting in the MiniBooNE fiducial volume according to MC. The SciBooNE selection criteria retains $37 \%$ (35\%) of all CC inclusive (CCQE) $\bar{\nu}$ events interacting in the SciBar fiducial volume according to MC.

MiniBooNE and SciBooNE reconstruction also accurately reproduces the antineutrino energy for selected CCQE events (and non-CCQE events to a lesser degree). The MiniBooNE selected sample has a $8.3 \%(13.9 \%)$ standard deviation on the Gaussian fit to the energy resolution for CCQE (all) events. The SciBooNE selected sample has a 9.6\% (24.6\%) standard deviation on the Gaussian fit to the energy resolution for CCQE (all) events. The antineutrino energy reconstruction will determine both the data and MC reconstructed antineutrino energy distributions, the comparison of which will ultimately test the theory proposed in this analysis. 


\section{Chapter 6}

\section{Uncertainties}

The purpose of this chapter is to characterize and quantify all the uncertainties that are associated with the analysis. All the uncertainties are then combined into a $42 \times 42$ covariance matrix (error matrix), which encodes the uncertainty of each reconstructed antineutrino energy bin in MiniBooNE and SciBooNE and its correlated uncertainties to all other reconstructed antineutrino energy bins in MiniBooNE and SciBooNE. The error matrix, with all the uncertainties encapsulated, will ultimately be used in the analysis fit to determine the final result.

The systematic uncertainties considered in the analysis can be divided into three major categories: antineutrino flux, neutrino interaction, and detector specific. Antineutrino flux uncertainties are uncertainties involving meson production and interaction at the Be target through magnetic horn focusing up to the neutrino beam flux. Neutrino interaction uncertainties includes neutrino cross section uncertainties and subsequent nuclear interaction uncertainties. If the underlying systematic uncertainty is common between MiniBooNE and SciBooNE, the systematic uncertainty is treated as correlated between MiniBooNE and SciBooNE. It should be noted that much of the flux and neutrino interaction uncertainties cancel in this analysis since they are common between MiniBooNE and SciBooNE. Detector specific uncertainties are associated with modeling efficiencies of particle propagation and observation in the detector including size and mass uncertainties, which are unique to each detector.

The chapter is organized as follows. The multsim method of handling correlated uncer- 
tainties is first described. Antineutrino flux and neutrino interaction uncertainties are then characterized and quantified. Detector uncertainties for both MiniBooNE and SciBooNE are then discussed. A short explanation on the treatment of statistical uncertainties follows. The description of the construction of the combined error matrix is next. Finally, the chapter concludes with the presentation of the combined error matrix, its properties, and some final comments.

\subsection{Multsim Method and Reweighting}

Antineutrino flux and neutrino interaction uncertainties are calculated for both MiniBooNE and SciBooNE using the multisim method [100], which handles a large number of correlated uncertainties. To understand the multisim method, it is necessary to understand how an uncertainty is characterized in an error matrix for uncorrelated uncertainties. Assume there is a single source of uncertainty in parameter $\alpha$ called $\delta_{\alpha}$ so that $\alpha \pm \delta_{\alpha}$. To understand the effect the uncertainty of $\alpha$ has on the antineutrino energy distribution, create two antineutrino energy distribution predictions, one with the default value of $\alpha, p_{i}^{\alpha}$, and one with $\alpha^{\prime}=\alpha+\delta_{\alpha}$, denoted $p_{i}^{\delta}$. The $p_{i}^{\alpha}\left(p_{i}^{\delta}\right)$ values are the correlated set of predicted number of events in each of the antineutrino energy bins for parameter $\alpha\left(\alpha^{\prime}\right)$. The elements of the covariance matrix, also called the error matrix, which represent the correlated uncertainties between energy bins $i$ and $j$ due to $\alpha \pm \delta_{\alpha}$ are:

$$
M_{i j}^{\alpha}=\left(p_{i}^{\alpha}-p_{i}^{\delta}\right)\left(p_{j}^{\alpha}-p_{j}^{\delta}\right)
$$

If $\alpha$ has no effect on the energy distribution, then $p_{i}^{\alpha}-p_{i}^{\delta}=0$ and the elements of the error matrix are all 0 . If all the uncertainties are uncorrelated, it is possible to vary each source of systematic uncertainty by one standard deviation and rerun the entire prediction with each change. This is called the unisim method. The combined error matrix is then just the sum of all the covariance matrices for the different systematic uncertainties.

In this analysis, many of the uncertainties are correlated. For example, the total amount of light detected in MiniBooNE is constrained by calibration measurements. Therefore, the scintillation light and Cherenkov light cannot both be increased simultaneously. A variation 
of the scintillation light within its uncertainty must imply a variation of the Cherenkov light for the sum to remain constant.

To account for correlations, the multisim method is used. The multisim method is an extension of the unisim method. Instead of varying one parameter $\alpha$, one varies a set of correlated parameters $\vec{\alpha}=\left(\alpha_{1}, \alpha_{2}, \ldots, \alpha_{N}\right)$ in a correlated way. For example, instead of varying scintillation and Cherenkov light individually, scintillation light and Cherenkov light would be varied within their individual errors in their allowed phase space region while requiring the total amount of light to be fixed.

The way $\vec{\alpha}$ can be varied in a correlated fashion is to Chelosky decompose the correlated covariance matrix of the parameters (the covariance matrix quantifies how the parameters are correlated). The decomposition is always possible since a covariance matrix is always at least positive semi-definite and therefore can always be closely approximated as positive definite by adding small, negligible terms to some of the elements if necessary. The Chelosky decomposition of the covariance matrix results in a product of a lower triangular matrix and its transpose. With the lower triangular matrix and a set of independent standard normally distributed random variables, $\vec{\alpha}$ can be varied in a correlated fashion within its parameter space.

Similar to the unisim method, a prediction $p^{k}$ is created for each draw $\vec{\alpha}_{k}$ from the allowed parameter space. For example, let the prediction $p^{k}$ be the antineutrino energy distribution. Each prediction $p^{k}$ represents a different antineutrino energy distribution corresponding to its draw $\vec{\alpha}_{k}$. With a large number of draws, the entire parameter space of $\vec{\alpha}$ can be covered and therefore all possible antineutrino energy distribution variations (the $p^{k}$ predictions) from the entire parameter space of $\vec{\alpha}$ are considered.

The error matrix which describes the effect of variations to $\vec{\alpha}$ within the allowed parameter space is:

$$
M_{i j}^{\vec{\alpha}}=\frac{1}{N-1} \sum_{k}^{N}\left(p_{i}^{c v}-p_{i}^{k}\right)\left(p_{j}^{c v}-p_{j}^{k}\right)
$$

where $N$ is the total number of draws used, $p^{c v}$ represents the central value prediction using the default set of $\vec{\alpha}$, and $p^{k}$ represents the varied prediction using draw $k$ of $\vec{\alpha}$. For a Gaussian distributed error of $\alpha \pm \delta_{\alpha}$ considered in the unisim case, one draw is sufficient; for other situations, more draws are required. The advantage of the multisim method is 
that it can be applied when $\vec{\alpha}$ is correlated, when the unisim method cannot be used. In the case of 1 parameter and 1 prediction value, 1000 draws would map out the effect on the prediction due to the parameter to the $\sim 3 \%$ level. More information can be found under Reference [100].

To run the multisim method(thousands of draws of $\vec{\alpha}$ ) would require substantial computing resources if the antineutrino flux and neutrino interaction $\mathrm{MC}$ predictions were rerun for each draw. To avoid this problem, the "reweighting" method is used to calculate a new weight for a default MC event, instead of generating an entirely new event. For all MC events, important underlying parameter information is stored (e.g. neutrino energy, distance traveled, cross section interaction type and kinematics, neutrino parent type and kinematics, muon kinematics). Now, instead of rerunning the entire simulation, a weight, $w t_{k}$, is applied to each event based on the change due to a particular $\vec{\alpha}$ throw. A weight is then applied for all events to form a new prediction. The weight is the effect of changing underlying parameters $\vec{\alpha}$ (e.g. the ratio of the cross section with a change to the axial mass, $\left.w t=\frac{\sigma\left(M_{A}=1.28\right)}{\sigma\left(M_{A}=1.35\right)}\right)$. Reweighting is used for the antineutrino flux and neutrino interaction systematics, and some detector systematics.

For each of the antineutrino flux and neutrino interaction uncertainties and some detector uncertainties, 1000 draws were used to form the error matrices to minimize both statistical limitations of the draws and computational time. The error matrix for the correlated uncertainties, binned in the relevant parameter(s), is Cholesky decomposed and its elements are used to determine the 1000 draws for reweighting. The 1000 draws for each event are then combined with the specific parameters of the event to find the 1000 variational weights for the event. The 1000 variational weights for each neutrino or antineutrino event, after combining for all events, correspond to 1000 antineutrino energy distribution predictions. For each uncertainty, as a binned function of reconstructed antineutrino energy, the 1000 predictions are combined with the central value prediction according to Equation 6.2 to form the energy bin error matrix of the specific uncertainty. Correlated uncertainties between MiniBooNE and SciBooNE are given the same 1000 reweighting draws for every MiniBooNE and SciBooNE event. Uncorrelated uncertainties between MiniBooNE and SciBooNE are given different 1000 reweighting draws between MiniBooNE and SciBooNE 
events. The error matrices for the separate uncertainties are ultimately combined to form the error matrix used in the fit of the analysis. Further discussion on error matrices can be found in Reference 100 .

\subsection{Antineutrino Flux Uncertainties}

The antineutrino flux uncertainties pertains to all relevant uncertainties up to and including the generation of the antineutrino flux. The sources of antineutrino flux uncertainties, in order of production, are:

1. p-Be hadronic cross sections for $\nu_{\mu}$ events

2. p-Be hadronic cross sections for $\bar{\nu}_{\mu}$ events

3. $\pi^{+}$production in the antineutrino beam flux

4. $\pi^{-}$production from initial p-Be interaction

5. $K^{+}$production from initial p-Be interaction

6. $K^{0}$ production from initial p-Be interaction

7. $K^{-}$production from initial $\mathrm{p}$-Be interaction

8. magnetic field and skin effect produced by the horn for $\nu_{\mu}$ events

9. magnetic field and skin effect produced by the horn for $\bar{\nu}_{\mu}$ events

The dominant antineutrino flux uncertainties are the $\pi^{+}$and $\pi^{-}$production uncertainties with a significant contribution coming from the magnetic horn-related uncertainties.

Through $\pi^{+} \rightarrow \nu_{\mu}+\mu^{+}$, parent $\pi^{+}$production cross section uncertainties are relevant in this $\bar{\nu}_{\mu}$ analysis due to the large fraction of selected background $\nu_{\mu}$ events. Parent $\pi^{+}$ production cross section uncertainties for both the initial p-Be interaction and subsequent hadronic interactions are constrained by direct measurements of $\nu_{\mu}$ in the antineutrino beam flux using MiniBooNE 101; 102. The beam fraction of $\nu_{\mu}$ in the antineutrino beam was determined using three methods: a pure data sample of $\nu_{\mu}$ events from $\mathrm{CC} 1 \pi$ interactions, differences in Michel electron rates between final state $\mu^{-}$and $\mu^{+}$from $\nu_{\mu}$ and $\bar{\nu}_{\mu}$ 
interactions, respectively, due to $\mu^{-}$capture on carbon, and angular distribution differences between final state $\mu^{-}$and $\mu^{+}$from $\nu_{\mu}$ and $\bar{\nu}_{\mu}$ interactions, respectively. Averaging these three methods, the $\pi^{+}$production in the beam MC was scaled by a factor of 0.78 and given a $12.8 \%$ normalization uncertainty. For this analysis, all parent $\pi^{+}$neutrino events in the antineutrino beam are given a $12.8 \%$ Gaussian distributed normalization uncertainty, regardless of its kinematic properties.

Parent $\pi^{-}$production cross section uncertainties from the initial p-Be interaction are calculated using the HARP experiment data and covariance matrix 103. A spline is created from the HARP data set to interpolate across relevant $p_{\pi^{-}}$and $\theta_{\pi^{-}}$values. Each parent $\pi^{-}$antineutrino event is weighted the ratio of $w t=\frac{\sigma_{\text {spline }}}{\sigma_{S W}} 1000$ times (corresponding to the 1000 draws), where $\sigma_{\text {spline }}$ is the new $\pi^{-}$cross section calculated from the spline and $\sigma_{S W}$ is the cross section according to the default Sanford-Wang parameterization [3, to determine the uncertainty of the parent $\pi^{-}$production uncertainty for a given event.

The parent $K^{+}$production cross section uncertainties from the initial p-Be interaction are formed by taking correlated draws from the Feynman scaling covariance matrix [48] based on the recent SciBooNE measurement 104. The weight of the parent $K^{+}$neutrino event for each draw is $w t=\frac{\sigma_{c_{i}^{k}}}{\sigma_{F S}}$, the ratio of the new cross section with the drawn $c_{i}^{k}$ Feynman parameterization to the default $\sigma_{F S}$ Feynman scaling prediction cross section.

The parent $K^{0}$ production cross section uncertainties from the initial p-Be interaction are formed by taking correlated draws from the Sanford-Wang parameterization covariance matrix 3. The weight for a parent $K^{0}$ neutrino event is $w t=\frac{\sigma_{c_{i}}}{\sigma_{S W}}$, the ratio of the new cross section with the drawn $c_{i}$ Sanford-Wang parameterization to the default $\sigma_{S W}$ Sanford-Wang prediction cross section. $K^{-}$production is estimated using the MARS hadronic interaction package [50] due to the scarcity of production measurements in the relevant kinematic regions and is not well understood so parent $K^{-}$antineutrino events are given a conservative $100 \%$ Gaussian distributed normalization uncertainty, regardless of its kinematic properties.

p-Be hadronic cross sections uncertainties include uncertainties involving the secondary nucleon and pion interactions in the Be target and $\mathrm{Al}$ magnetic horn after initial parent pion production. The magnetic field uncertainty can be described by an uncertainty in the magnetic horn current of $\pm 1 \mathrm{kA}$. The uncertainty in the skin effect of the Al magnetic horn 
can be described by an uncertainty in the skin depth of $\pm 1.4 \mathrm{~mm}$. The p-Be hadronic cross section, horn magnetic field and skin effect uncertainties are quantified by rerunning the beam simulation for each lower and upper one standard deviation change and producing a series of histograms as a function of neutrino energy, radial distance away from center of beam z-axis, and neutrino type for each simulation. The series of histograms are combined to form an error matrix of the uncertainties as a function of neutrino energy and radial distance using the unisim method. Draws from this neutrino energy and radial distance binned error matrix are used to form the systematic predictions for the p-Be hadronic cross section, horn magnetic field and skin effect uncertainties. Separate error matrices are produced for the uncertainties of $\nu_{\mu}$ events and $\bar{\nu}_{\mu}$ events.

Figure 6.1 shows the flux error matrix and its correlations as a function of reconstructed antineutrino energy bins in MiniBooNE and SciBooNE. The limits of the 21 reconstructed antineutrino energy bins, which are the same for both MiniBooNE and SciBooNE, are listed in Table 6.2. Bins 1-21 are MiniBooNE and bins 22-42 are SciBooNE.

\subsection{Neutrino Interaction Uncertainties}

The neutrino interaction uncertainties includes all relevant uncertainties that involve the initial neutrino-nuclear interaction, such as cross section uncertainties, and subsequent nuclear interaction modeling. The cross section uncertainties are represented by uncertainties in the axial mass of the neutrino-nucleus dipole model used to calculate the cross sections. The sources of neutrino interaction uncertainties are:

1. charged current quasielastic axial mass on carbon target

2. $\kappa$, the Pauli blocking parameter

3. charged current quasielastic axial mass on hydrogen target

4. charged current resonant $\pi$ axial mass

5. charged current coherent $\pi$ axial mass

6. charged current multi $\pi$ axial mass 


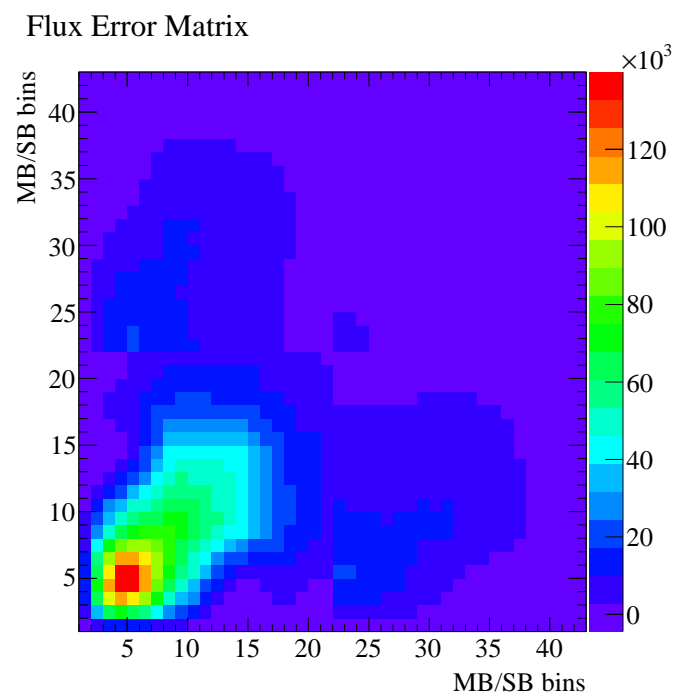

(a) Flux Error Matrix
Flux Correlation Error Matrix

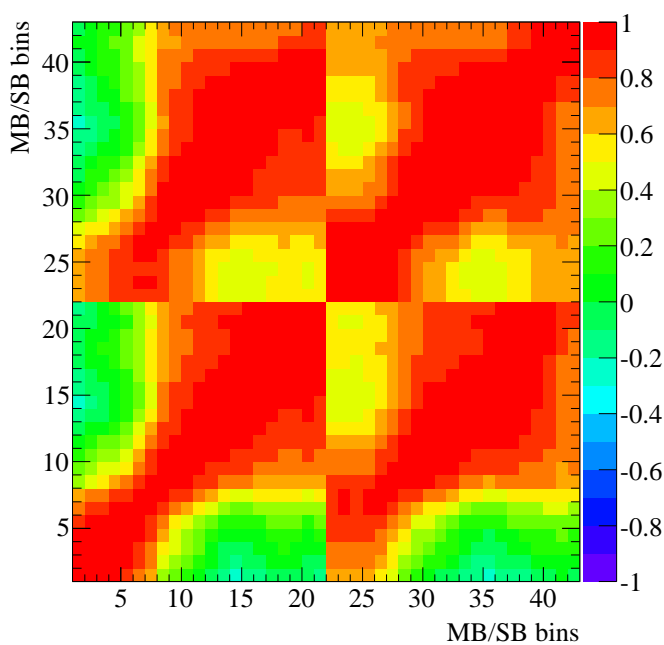

(b) Correlations of Flux Error Matrix

Figure 6.1: The MiniBooNE-SciBooNE flux error matrix (left) and its correlation (right) are shown as a function of reconstructed antineutrino energy bins with bin limits listed in Table 6.2. The bin limits are the same for both MiniBooNE and SciBooNE with bins 1-21 for MiniBooNE and bins 22-42 for SciBooNE.

7. nuclear binding energy

8. Fermi surface momentum

9. neutral current axial vector isoscalar contribution

10. limitations on the Relativistic Fermi Gas model to describe subsequent nuclear interactions after initial neutrino-nuclear interaction

11. $\pi$ absorption in nucleus

12. $\pi$ inelastic scattering

13. $\pi$-less $\Delta$ decay

The dominant neutrino uncertainties are the uncertainties associated with charged current quasielastic (CCQE) axial mass $M_{A}$ and with the limitations of the Relativistic Fermi 
Gas (RFG) model. The cross section and nuclear modeling uncertainties for $\nu$ and $\bar{\nu}$ events are set uncorrelated due to the poor understanding of differences between $\nu$ and $\bar{\nu}$ interactions in the neutrino-nucleus dipole model and their subsequent nuclear modeling. All the cross section and the nuclear modeling uncertainties are determined using the multisim method with 1000 draws due to correlations between some of the uncertainties and the relative ease of implementation.

CCQE cross sections on carbon are calculated assuming an RFG model with parameters $M_{A}=1.35 \mathrm{GeV} / \mathrm{c}^{2}$ and $\kappa=1.007$, where $\kappa$ is an empirical Pauli blocking parameter introduced to allow an extra degree of freedom to describe the MiniBooNE data at low momentum transfer. Based on the latest MiniBooNE CCQE neutrino cross section measurement on carbon [52, $M_{A}$ and $\kappa$ are given uncertainties of $\pm 0.07 \mathrm{GeV} / \mathrm{c}^{2}$ and \pm 0.005 , respectively, for CCQE interactions on carbon for both $\nu$ and $\bar{\nu}$ events separately. The uncertainties in $M_{A}$ and $\kappa$ for CCQE events on carbon are based only on the statistical uncertainties of the MiniBooNE CCQE neutrino cross section measurement, to avoid double counting sources of systematic uncertainties already accounted for in this analysis. Both the $\nu$ and $\bar{\nu} M_{A}$ central values of $1.014 \mathrm{GeV} / \mathrm{c}^{2}$ and their uncertainties of $\pm 0.014 \mathrm{GeV} / \mathrm{c}^{2}$ for quasielastic interactions on hydrogen are based on the latest deuterium measurements [81.

The default MC has a correction, as a function of $Q^{2}$, applied to background $\mathrm{CC} 1 \pi$ interaction events in MC based on the MiniBooNE CCQE neutrino cross section measurement [52]. The purpose of the $Q^{2}$ correction in the MiniBooNE measurement was to match the background $\mathrm{CC} 1 \pi$ interaction events in $\mathrm{MC}$ to a selected data sample comprising mainly of $\mathrm{CC} 1 \pi$ interaction events. Therefore, in light of the MC-to-data matching $Q^{2}$ matching, there is no uncertainty placed on $M_{A}$ for $\nu_{\mu} \mathrm{CC} 1 \pi$ interaction events. However, for $\bar{\nu}_{\mu} \mathrm{CC} 1 \pi$ interaction events, the $M_{A}$-resonant and coherent $1 \pi$ uncertainties are not constrained by the MiniBooNE measurement since the relationship between $\nu$ and $\bar{\nu}$ interactions is not well understood. These two interactions are given a $\pm 0.275 \mathrm{GeV} / \mathrm{c}^{2}$ uncertainty for $M_{A}$ consistent with previous MiniBooNE and SciBooNE measurements 105 . 37 .

$M_{A}$ for multi $\pi$ interactions are given an uncertainty of $\pm 0.52 \mathrm{GeV} / \mathrm{c}^{2}$. The Fermi surface momentum $\left(p_{F}\right)$ of carbon nuclei are given $\pm 30 \mathrm{MeV} / \mathrm{c}$ uncertainty and the nuclear 
binding energy $\left(E_{B}\right)$ of carbon nuclei are given $\pm 9 \mathrm{MeV}$ uncertainty. The $\mathrm{NC}$ axial vector isoscalar contribution $(\Delta \mathrm{s})$ is given an uncertainty of \pm 0.1 . These uncertainties are identical to previous MiniBooNE and SciBooNE measurements [105, 37.

Pion absorption, pion inelastic scattering, and pionless $\Delta$ decay in the carbon nucleus are given $\pm 25 \%, \pm 30 \%$, and $\pm 100 \%$ Gaussian distributed normalization uncertainties, respectively. These uncertainties are very small compared to other neutrino interaction uncertainties and are uncorrelated between MiniBooNE and SciBooNE events, unlike all other neutrino interaction uncertainties, due to difficulties in correlating them. These uncertainties are not included in Figure 6.3 in this section, but are included in Figures 6.4 and 6.5 in Section 6.4 due to their separate calculational methods for MiniBooNE and SciBooNE.

Nuclear interaction modeling systematic uncertainties are added to account for the limitations of the RFG model of Smith and Moniz 84 . Such limitations include the absence of processes such as meson exchange currents and multi-nucleon knockout events $106 ; 107 ; 108$; 109. Since these are RFG-related uncertainties, they are applied only to the neutrino and antineutrino interactions on carbon nuclei. Figure 6.2 shows how other recent nuclear models compare to the RFG model with a CCQE $M_{A}=1.35 \mathrm{GeV} / \mathrm{c}^{2}$ and $\kappa=1.007$. The largest discrepancy comes from Reference [106]. All the nuclear models in the figure were calculated under the assumption that the initial neutrino-nucleus interaction is CCQE. A 40\% Gaussian distributed normalization uncertainty is placed on $\bar{\nu}$ CCQE interactions on carbon to cover the discrepancy between the RFG model prediction for $\bar{\nu}$ and recent nuclear models 106 ; $107 ; 108 ; 109$. An additional 10\% Gaussian distributed normalization uncertainty is added to non-CCQE $\bar{\nu}$ interactions on carbon to account for the limitations of the RFG model for those type of events. Furthermore, an additional 10\% Gaussian distributed normalization uncertainty is assigned to both $\nu$ and $\bar{\nu}$ CCQE interactions on carbon to cover the difference between data and prediction in the MiniBooNE CCQE neutrino cross section measurement.

Figure 6.3 shows the neutrino interaction error matrix and its correlations as a function of reconstructed antineutrino energy bins in MiniBooNE and SciBooNE. The limits of the 21 reconstructed antineutrino energy bins, which are the same for both MiniBooNE and SciBooNE, are listed in Table 6.2. Bins 1-21 are MiniBooNE and bins 22-42 are SciBooNE. 


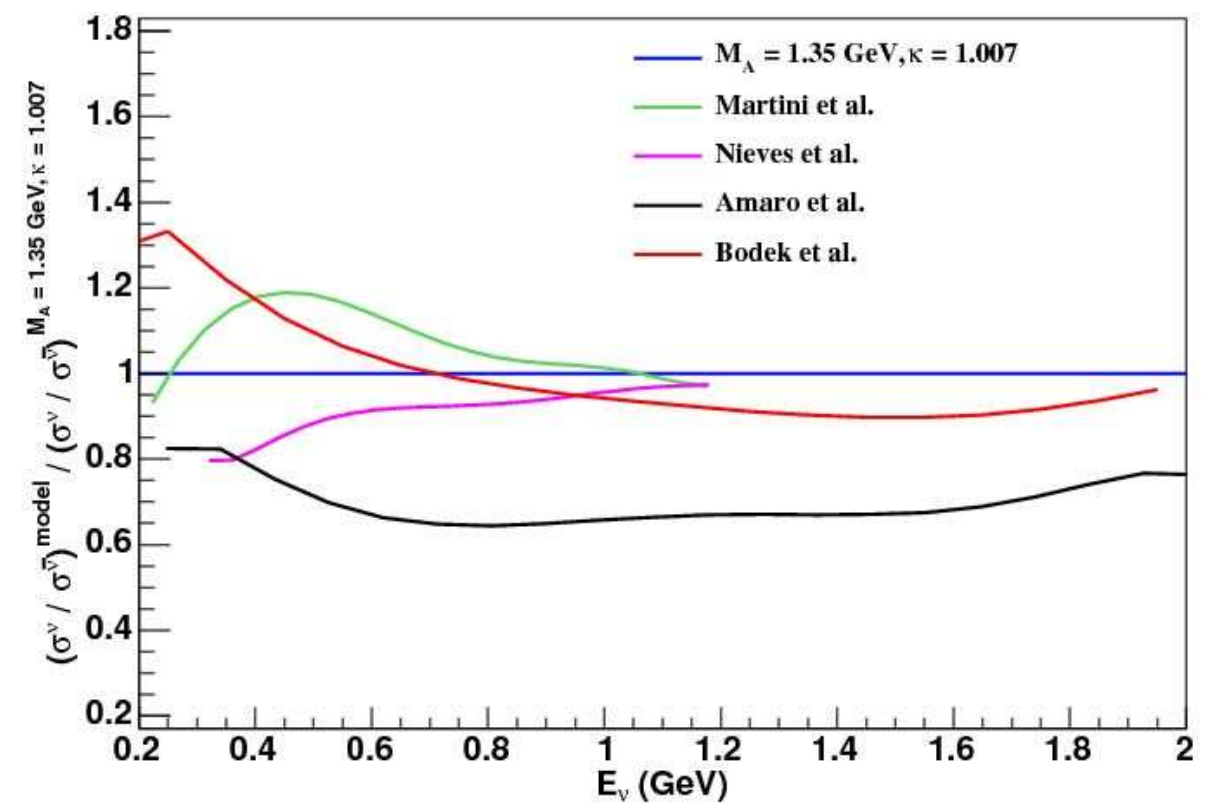

Figure 6.2: The ratio of neutrino cross section to antineutrino cross section $\left(\sigma^{\nu} / \sigma^{\bar{\nu}}\right)$ for the various nuclear models relative to neutrino cross section to antineutrino cross section $\left(\sigma^{\nu} / \sigma^{\bar{\nu}}\right)$ for the RFG model with CCQE $M_{A}=1.35 \mathrm{GeV} / \mathrm{c}^{2}$ and $\kappa=1.007$ as a function of (anti)neutrino energy. CCQE for the initial neutrino-nucleus interaction is assumed.

The full list of antineutrino beam flux and neutrino interactions parameters for the MC simulation and its associated systematic uncertainties are shown in Table 6.1.

\subsection{Detector Uncertainties}

MiniBooNE, an oil Cherenkov detector, and SciBooNE, a discrete scintillator detector, use fundamentally different neutrino detection methods so their detector uncertainties are different and uncorrelated.

\subsubsection{SciBooNE Detector Uncertainties}

The SciBooNE detector uncertainties are calculated with the unisim method. Two separate MC prediction are produced for each SciBooNE detector uncertainty, one for the default value up one standard deviation and one for the default value down one standard deviation. 
Table 6.1: Summary of antineutrino beam flux and neutrino interaction parameters for MC simulation with its associated systematic uncertainties.

\begin{tabular}{|c|c|}
\hline Beam & Uncertainty \\
\hline$\pi^{+}$production in antineutrino beam & $12.8 \%$ normalization uncertainty 101 \\
\hline$\pi^{-}$production from $\mathrm{p}$-Be interaction & Spline fit to HARP data \\
\hline$K^{+}$production from p-Be interaction & Table IX in Ref. $48 \mid$ \\
\hline$K^{0}$ production from $\mathrm{p}$-Be interaction & Table IX in Ref. $|3|$ \\
\hline$K^{-}$production from $\mathrm{p}$-Be interaction & $100 \%$ normalization uncertainty \\
\hline Nucleon and pion interaction in $\mathrm{Be} / \mathrm{Al}$ & Table XIII in Ref. $3 \mid$ \\
\hline Horn current & $\pm 1 \mathrm{kA}$ \\
\hline Horn skin effect & Horn skin depth, $\pm 1.4 \mathrm{~mm}$ \\
\hline Cross Sections & Uncertainty \\
\hline CCQE $M_{A}$ on carbon target & $1.35 \pm 0.07 \mathrm{GeV} / \mathrm{c}^{2}$ \\
\hline$\kappa$ & $1.007 \pm 0.005$ \\
\hline CCQE $M_{A}$ on hydrogen target & $1.014 \pm 0.014 \mathrm{GeV} / \mathrm{c}^{2}$ \\
\hline CC resonant $\pi M_{A}$ & $1.1 \pm 0.275 \mathrm{GeV} / \mathrm{c}^{2}[1]$ \\
\hline CC coherent $\pi M_{A}$ & $1.03 \pm 0.275 \mathrm{GeV} / \mathrm{c}^{2}[1]$ \\
\hline CC multi $\pi M_{A}$ & $1.3 \pm 0.52 \mathrm{GeV} / \mathrm{c}^{2}$ \\
\hline$E_{B}$ & $\pm 9 \mathrm{MeV}$ \\
\hline$p_{F}$ & $220 \pm 30 \mathrm{MeV} / \mathrm{c}$ \\
\hline$\Delta \mathrm{s}$ & $0.0 \pm 0.1$ \\
\hline CCQE on carbon & $\pm 10 \%$ norm error \\
\hline CCQE on carbon $\left(\bar{\nu}_{\mu}\right)$ only & $\pm 40 \%$ norm error \\
\hline non-CCQE on carbon $\left(\bar{\nu}_{\mu}\right)$ only & $\pm 10 \%$ norm error \\
\hline$\pi$ absorption in nucleus & $25 \%$ \\
\hline$\pi$ inelastic scattering & $30 \%$ \\
\hline$\pi$-less $\Delta$ decay & $100 \%$ \\
\hline
\end{tabular}

1 This uncertainty is not applied to $\nu_{\mu} \mathrm{CC} 1 \pi$ events that are $Q^{2}$ corrected. 


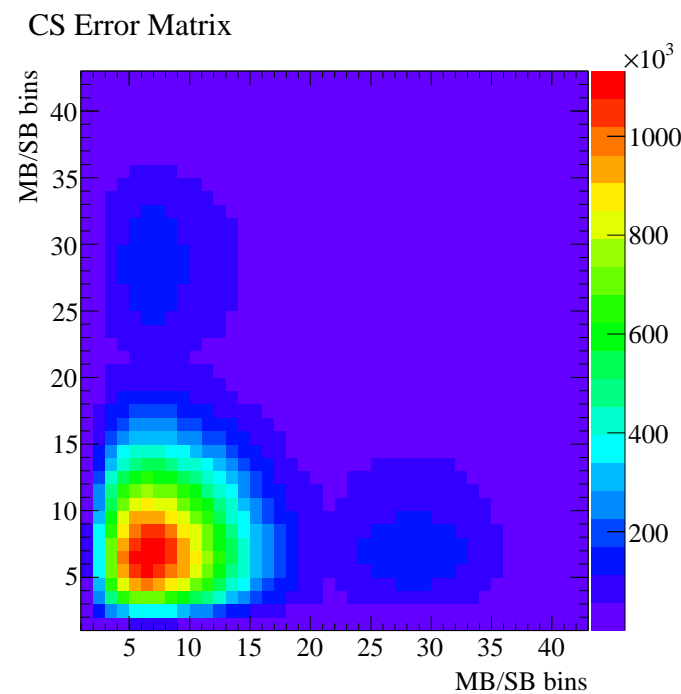

(a) Cross section Error Matrix
CS Correlation Error Matrix

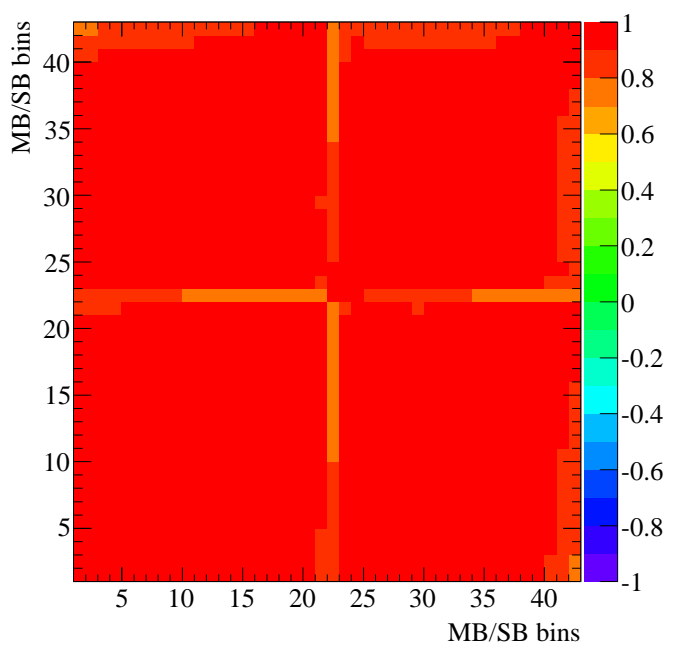

(b) Correlations of Cross section Error Matrix

Figure 6.3: The MiniBooNE-SciBooNE neutrino interaction error matrix (left) and its correlation (right) are shown as a function of reconstructed antineutrino energy bins with bin limits listed in Table 6.2. The bin limits are the same for both MiniBooNE and SciBooNE with bins 1-21 for MiniBooNE and bins 22-42 for SciBooNE.

For each uncertainty, as a binned function of reconstructed antineutrino energy, the up and down standard deviation MC predictions are individually compared to the default prediction, its two resulting error matrices averaged to form the error matrix for the uncertainties. The SciBooNE specific uncertainties include the detector uncertainties, reconstruction uncertainties, and background contamination uncertainties.

The SciBooNE detector uncertainties consist of uncertainties in the muon energy loss in the scintillator and iron, light attenuation in the wavelength shifting fibers, and PMT response; see Reference [105]. The crosstalk of the MA-PMT was measured to be $3.15 \%$ for adjacent channels, with an absolute error of $0.4 \%$ [41]. The single photoelectron resolution of the MA-PMT is set to $50 \%$ in the simulation, and the absolute error is estimated to be $\pm 20 \%$. Birk's constant for the SciBar scintillator was measured to be $0.0208 \pm 0.0023$ $\mathrm{cm} / \mathrm{MeV}$ [41]. The conversion factors from the ADC counts to the photoelectron were measured for all 14,336 MA-PMT channels in SciBar. The measurement uncertainty was 
at the $20 \%$ level. The threshold for hits to be used in SciBar track reconstruction is 2.5 photoelectrons; this threshold is varied by $\pm 20 \%$ to evaluate the systematic error for SciBar track reconstruction. The TDC dead time is set to $55 \mathrm{~ns}$ in the MC simulation, with the error estimated to be \pm 20 ns 51 .

The SciBooNE reconstruction uncertainties consist of antineutrino energy reconstruction uncertainties and muon track misidentification uncertainties. Since the antineutrino energy is reconstructed from the muon momentum assuming CCQE kinematics, systematic uncertainties in the muon momentum measurement is propagated into uncertainties in the reconstructed antineutrino energy. For antineutrino energy reconstruction uncertainties, the densities of SciBar, EC, and MRD are varied independently within their measured uncertainties of $\pm 3 \%, \pm 10 \%$, and $\pm 3 \%$, respectively. Misidentified muons stem mainly from proton tracks created through NC interactions, which are given a conservative $\pm 20 \%$ normalization uncertainty. A conservative $\pm 20 \%$ normalization uncertainty is applied for the MC simulated background of neutrino and antineutrino events initially interacting outside the SciBooNE detector that pass the selection criteria. A conservative $\pm 20 \%$ normalization uncertainty is applied for the MC simulated background of neutrino and antineutrino events initially interacting in the EC/MRD detector that pass the selection criteria.

Figure 6.4 shows the SciBooNE detector error matrix and its correlations as a function of reconstructed antineutrino energy bins with bin limits listed in Table 6.2. The 21 SciBooNE antineutrino energy bins have the exact same limits as the SciBooNE antineutrino energy bins in the MiniBooNE and SciBooNE error matrices for other uncertainties. Note that the pion absorption, pion inelastic scattering, and pionless $\Delta$ decay uncertainties in the carbon nucleus from Section 7.5 for the SciBooNE detector are included in the figure.

\subsubsection{MiniBooNE Detector Uncertainties}

Uncertainties associated with the MiniBooNE detector can be divided into two main categories: optical model and PMT response. The optical model includes light propagation, attenuation, and scattering (Rayleigh and Raman) in the detector. The optical model in the detector 94 uses 35 parameters for properties such as refractive index, attenuation

length, scintillation strength, reflections, fluorescence, etc. These parameters are tuned to 


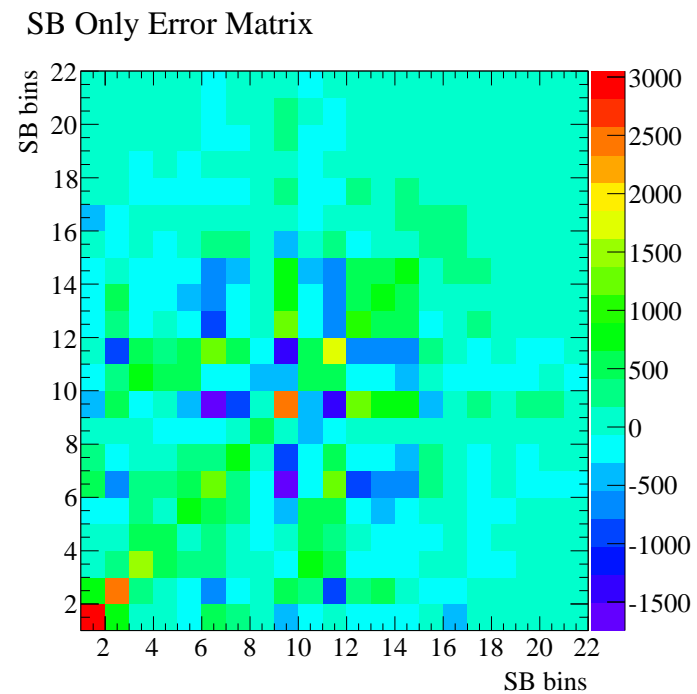

(a) SB Detector Error Matrix
SB Only Correlation Error Matrix

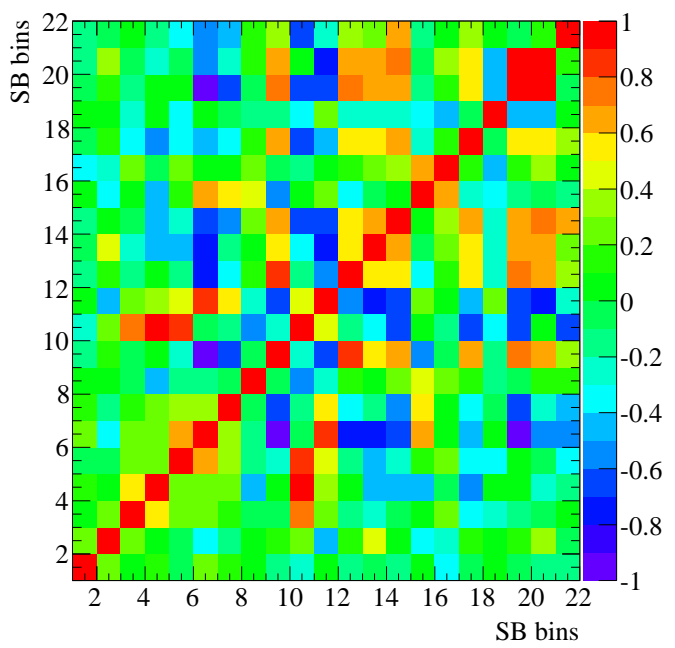

(b) Correlations of SB Detector Error Matrix

Figure 6.4: The SciBooNE detector error matrix (left) and its correlation (right) are shown as a function of SciBooNE reconstructed antineutrino energy bins with bin limits listed in Table 6.2. The 21 SciBooNE antineutrino energy bins presented here have the exact same limits as the SciBooNE antineutrino energy bins in the MiniBooNE and SciBooNE error matrices for other uncertainties.

non-MiniBooNE measurements as well as MiniBooNE internal data. 120 separate MC data sets from correlated changes of the 35 parameters in the form of histograms were created based on variations in these parameters. Histograms created from the optical model variations are smoothed to eliminate the contribution of statistical errors from the MC. Using the multisim method, these variations are then used to compute the optical model error matrix in bins of reconstructed antineutrino energy.

To estimate the impact of uncertainties in PMT response, independent MC data sets based on variations in the discriminator threshold, or the PMT charge-time correlations, were created and compared to the default MC data set. Based on comparisons with external data 110, 111, 112 and the output of GCALOR, a MC detector simulation package, an uncertainty of $35 \%$ is assigned to pion absorption and $50 \%$ is assigned to charge exchange in the detector medium. This is distinct from the uncertainties on pion absorption $( \pm 25 \%)$ 
and charge exchange $( \pm 35 \%)$ inside the carbon nucleus for nuclear interaction modeling in Section 7.5 ,

Figure 6.5 shows the MiniBooNE detector error matrix and its correlations as a function of reconstructed antineutrino energy bins with bin limits listed in Table 6.2. The 21 MiniBooNE antineutrino energy bins have the exact same limits as the MiniBooNE antineutrino energy bins in the MiniBooNE and SciBooNE error matrices for other uncertainties. Note that the pion absorption, pion inelastic scattering, and pionless $\Delta$ decay uncertainties in the carbon nucleus from Section 7.5 for the MiniBooNE detector are included in the figure.

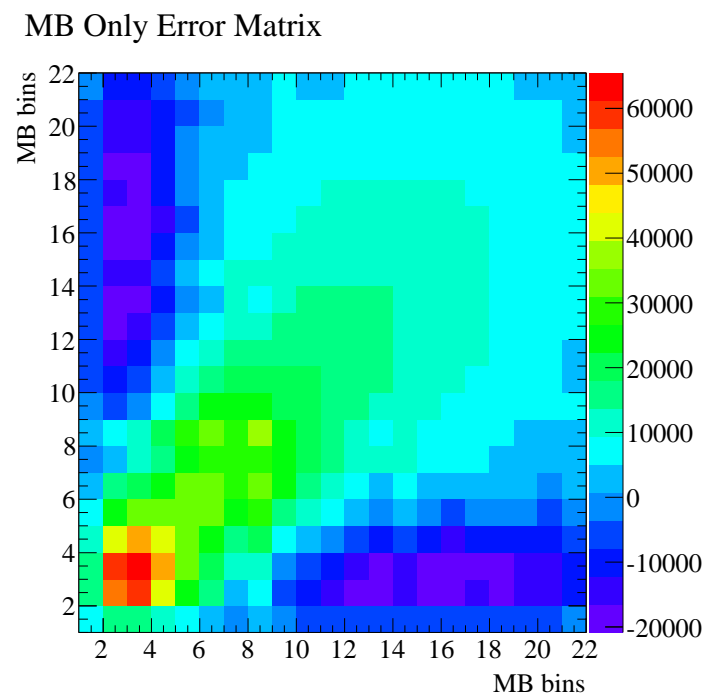

(a) MB Detector Error Matrix
MB Only Correlation Error Matrix

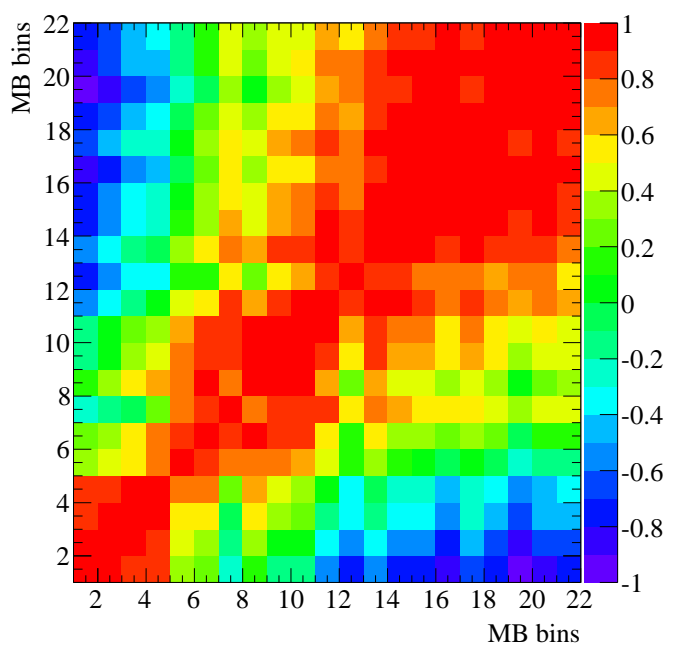

(b) Correlations of MB Detector Error Matrix

Figure 6.5: The MiniBooNE detector error matrix (left) and its correlation (right) are shown as a function of MiniBooNE reconstructed antineutrino energy bins with bin limits listed in Table 6.2. The 21 MiniBooNE antineutrino energy bins presented here have the exact same limits as the MiniBooNE antineutrino energy bins in the MiniBooNE and SciBooNE error matrices for other uncertainties. 


\subsection{Statistical Uncertainties}

The selected events are assumed to occur at a fixed rate and independently of each other so they follow a Poisson distribution. The $\sigma$ of the statistical uncertainties are the square root of the number of events observed. This leads to a diagonal covariance matrix with 42 elements (21 MiniBooNE elements and 21 SciBooNE elements with bin limits listed in Table 6.2 along the diagonal.

\subsection{Error Matrix}

The purpose of the error matrix is to encode all the uncertainties in the form of correlated uncertainties in reconstructed antineutrino energy since it is ultimately the comparison between data and $\mathrm{MC}$ prediction in reconstructed antineutrino energy that will be used to test for oscillations and antineutrino disappearance. The error matrix used in the fit of this analysis is formed by combining all the separate error matrices of MiniBooNE and SciBooNE from the different uncertainties. For this reason, all the error matrices for MiniBooNE and SciBooNE are all binned as a function of reconstructed antineutrino energy with the same bin limits.

\subsubsection{Fractional Error Matrix}

The elements of the error matrix are the covariances of the reconstructed antineutrino energy bins so they depend on the MC predicted value in the reconstructed antineutrino energy bins. Since the MC predicted value in each reconstructed antineutrino energy bin changes as a function of the oscillation parameters being tested in the physics parameter space, the error matrix must also be a function of the oscillation parameters in the physics

parameter space. Therefore, the error matrix must be constructed in such a way that its elements change as the oscillation parameters change. This construction requires that the total error matrix of all the uncertainties be constructed from fractional error matrices and the number of MC predicted $\bar{\nu}_{\mu}$ (right-sign) and $\nu_{\mu}$ (wrong-sign) events based on the oscillation parameter values.

The total error matrix is formed by combining right-sign, wrong-sign, and mixed-sign 
fractional error matrices. First, the fractional error matrix must be understood. In general, the elements of the fractional error matrix are defined as:

$$
\hat{M}_{i j}=\frac{M_{i j}}{N_{i} N_{j}}
$$

where $\hat{M}_{i j}$ is the $i j$-th element of the fractional error matrix, $M_{i j}$ is the $i j$-th element of the corresponding error matrix, and $N_{i}\left(N_{j}\right)$ is the number of events in the $i$-th $(j$-th) bin, respectively.

With the fractional error matrix defined and explained, the right-sign, wrong-sign, mixed-sign fractional error matrices can now be defined. For the right-sign fractional error matrix, the elements are defined as:

$$
\hat{M}_{i, j ;(R S, R S)}=\frac{M_{i, j ;(R S, R S)}}{N_{i}^{R S} N_{j}^{R S}}
$$

where $\hat{M}_{i, j ;(R S, R S)}$ is the $i j$-th element of the right-sign fractional error matrix, $M_{i, j ;(R S, R S)}$ is the $i j$-th element of the corresponding right-sign error matrix (the covariance matrix between antineutrino events and antineutrino events in the bins), and $N_{i}^{R S}\left(N_{j}^{R S}\right)$ is the number of antineutrino events in the $i$-th $(j$-th) bin, respectively. The right-sign fractional error matrix can therefore be viewed as the covariance matrix if there is a single antineutrino event in all the $i$ and $j$ bins. For the wrong-sign fractional error matrix, the elements are defined as:

$$
\hat{M}_{i, j ;(W S, W S)}=\frac{M_{i, j ;(W S, W S)}}{N_{i}^{W S} N_{j}^{W S}}
$$

where $\hat{M}_{i, j ;(W S, W S)}$ is the $i j$-th element of the wrong-sign fractional error matrix, $M_{i, j ;(W S, W S)}$ is the $i j$-th element of the corresponding wrong-sign error matrix (the covariance matrix between neutrino events and neutrino events in the bins), and $N_{i}^{W S}\left(N_{j}^{W S}\right)$ is the number of neutrino events in the $i$-th $(j$-th) bin, respectively.

For the mixed-sign fractional error matrix, the elements are defined as:

$$
\hat{M}_{i, j ;(R S, W S)}=\frac{M_{i, j ;(R S, W S)}}{N_{i}^{R S} N_{j}^{W S}}
$$

where $\hat{M}_{i, j ;(R S, W S)}$ is the $i j$-th element of the mixed-sign fractional error matrix, $M_{i, j ;(R S, W S)}$ is the $i j$-th element of the corresponding mixed-sign error matrix (the covariance matrix 
between antineutrino events in the $i$ bins and neutrino events in the $j$ bins), $N_{i}^{R S}$ is the number of antineutrino events in the $i$-th bin, and $N_{j}^{W S}$ is the number of neutrino events in the $j$-th bin. Similarly, $\hat{M}_{i, j ;(W S, R S)}$ is the $i j$-th element of the mixed-sign error matrix defined relative to $M_{i, j ;(W S, R S)}$, the $i j$-th element of the mixed-sign error matrix between neutrino events in the $i$ bins and antineutrino events in the $j$ bins. $\hat{M}_{i, j ;(W S, R S)}$ and $M_{i, j ;(W S, R S)}$ are the transpose of matrices $\hat{M}_{i, j ;(R S, W S)}$ and $M_{i, j ;(R S, W S)}$, respectively.

The total error matrix, $M$, is a $42 \times 42$ covariance matrix in MiniBooNE and SciBooNE reconstructed antineutrino energy bins and has the following form:

$$
M=\left(\begin{array}{cc}
M^{\mathrm{MB}-\mathrm{SB}} & M^{\mathrm{SB}-\mathrm{SB}} \\
M^{\mathrm{MB}-\mathrm{MB}} & M^{\mathrm{SB}-\mathrm{MB}}
\end{array}\right) .
$$

Starting from the bottom left corner and proceeding up or right are 21 reconstructed antineutrino energy bins of MiniBooNE arranged according to increasing energy followed by the 21 reconstructed antineutrino energy bins of SciBooNE arranged according to increasing energy. The limits of the 21 reconstructed antineutrino energy bins for both MiniBooNE and SciBooNE are shown in Table6.2, The error matrix can be divided into four $21 \times 21$ matrix quarters: the bottom left quarter $\left(M^{\mathrm{MB}-\mathrm{MB}}\right)$ is the MiniBooNE to MiniBooNE antineutrino energy bin correlations, the top right quarter $\left(M^{\mathrm{SB}-\mathrm{SB}}\right)$ is the SciBooNE to SciBooNE antineutrino energy bin correlations, the top left quarter $\left(M^{\mathrm{MB}-\mathrm{SB}}\right)$ is the MiniBooNE to SciBooNE antineutrino energy bin correlations, and the bottom right quarter $\left(M^{\mathrm{SB}-\mathrm{MB}}\right)$ is the SciBooNE to MiniBooNE antineutrino energy bin correlations. The antineutrino flux and neutrino interaction uncertainties contribute to all the energy bins of the entire $42 \times$ 42 error matrix. The MiniBooNE detector uncertainties contribute to the bottom left $21 \times$ 21 quarter of the total error matrix. The SciBooNE detector uncertainties contribute to the top right $21 \times 21$ quarter of the total error matrix.The statistical uncertainties contribute only to the diagonal energy bins starting at the bottom left corner and ending at the top right corner.

The bin to bin covariance elements of the total error matrix are defined as:

$$
\begin{aligned}
M_{i, j}^{\mathrm{X}}= & \hat{M}_{i, j ;(R S, R S)}^{\mathrm{X}} N_{i}^{\mathrm{Y} R S} N_{j}^{\mathrm{Z} R S}+\hat{M}_{i, j ;(W S, W S)^{\mathrm{X}} N_{i}^{\mathrm{Y} W S} N_{j}^{\mathrm{Z} W S}} \\
& +\hat{M}_{i, j ;(R S, W S)}^{\mathrm{X}} N_{i}^{\mathrm{Y} R S} N_{j}^{\mathrm{Z} W S}+\hat{M}_{i, j ;(W S, R S)}^{\mathrm{X}} N_{i}^{\mathrm{Y} W S} N_{j}^{\mathrm{Z} R S}+M_{i, j}^{\mathrm{X} s t a t} .
\end{aligned}
$$


Table 6.2: Reconstructed antineutrino energy bin limits for MiniBooNE and SciBooNE.

\begin{tabular}{c|c|c}
\hline \hline Bin Number & Bin Lower Limit $(\mathrm{MeV})$ & Bin Upper Limit $(\mathrm{MeV})$ \\
\hline 1 & 300 & 400 \\
2 & 400 & 467 \\
3 & 467 & 533 \\
4 & 533 & 600 \\
5 & 600 & 667 \\
6 & 667 & 733 \\
7 & 733 & 800 \\
8 & 800 & 867 \\
9 & 867 & 933 \\
10 & 933 & 1000 \\
11 & 1000 & 1067 \\
12 & 1067 & 1133 \\
13 & 1133 & 1200 \\
14 & 1200 & 1267 \\
15 & 1267 & 1333 \\
16 & 1333 & 1400 \\
17 & 1400 & 1467 \\
18 & 1467 & 1533 \\
19 & 1533 & 1900 \\
20 & 1600 & \\
21 & & \\
\hline \hline
\end{tabular}

X denotes the type of detector correlation (either MB-MB, SB-SB, SB-MB, or MB-SB) with $\mathrm{Y}$ and $\mathrm{Z}$ denoting the type of bins (either MiniBooNE or SciBooNE) associated with X. For MiniBooNE to MiniBooNE correlations, $\mathrm{X}=\mathrm{MB}-\mathrm{MB}, \mathrm{Y}=\mathrm{MB}, \mathrm{Z}=\mathrm{MB}$. For SciBooNE to SciBooNE correlations, $\mathrm{X}=\mathrm{SB}-\mathrm{SB}, \mathrm{Y}=\mathrm{SB}, \mathrm{Z}=\mathrm{SB}$. For MiniBooNE to SciBooNE correlations, $\mathrm{X}=\mathrm{MB}-\mathrm{SB}, \mathrm{Y}=\mathrm{MB}, \mathrm{Z}=\mathrm{SB}$. For SciBooNE to MiniBooNE correlations, $\mathrm{X}=\mathrm{SB}-\mathrm{MB}, \mathrm{Y}=\mathrm{SB}$, 
$\mathrm{Z}=\mathrm{MB} \cdot N_{i}^{\mathrm{Y} R S}\left(N_{j}^{\mathrm{Z} R S}\right)$ and $N_{i}^{\mathrm{Y} W S}\left(N_{j}^{\mathrm{Z} W S}\right)$ are the number of right-sign antineutrino and wrong-sign neutrino events for bin type $\mathrm{Y}$ (bin type $\mathrm{Z}$ ) in reconstructed antineutrino energy bin $i$ (bin $j$ ), respectively. $\hat{M}_{i, j ;(R S, R S)}^{\mathrm{X}}$ are the elements of the right-sign fractional error matrix (antineutrino to antineutrino correlated) for detector correlation type X. $\hat{M}_{i, j ;(W S, W S)}^{\mathrm{X}}$ are the elements of the wrong-sign fractional error matrix (neutrino to neutrino correlated) for detector correlation type X. $\hat{M}_{i, j ;(R S, W S)}^{\mathrm{X}}$ and $\hat{M}_{i, j ;(W S, R S)}^{\mathrm{X}}$ are the elements of the mixed-sign fractional error matrix for antineutrino to neutrino correlated and neutrino to antineutrino correlated, respectively, with detector correlation type $\mathrm{X} . M^{\mathrm{X}}$ stat is the statistical covariance matrix in reconstructed antineutrino energy bins for correlation type $\mathrm{X}$ (only SB-SB and MB-MB have nonzero elements).

Figure 6.6 shows the right-sign fractional error matrix and the wrong-sign fractional error matrix for flux uncertainties in MiniBooNE and SciBooNE. Figure 6.7 shows the right-sign fractional error matrix and the wrong-sign fractional error matrix for neutrino interaction uncertainties in MiniBooNE and SciBooNE. Figure 6.8 shows the right-sign fractional error matrix and the wrong-sign fractional error matrix for detector uncertainties in SciBooNE. Figure 6.9 shows the right-sign fractional error matrix and the wrong-sign fractional error matrix for detector uncertainties in MiniBooNE. Finally, Figure 6.10 shows the total error matrix and its correlations as a function of reconstructed antineutrino energy bins in MiniBooNE and SciBooNE. For all the figures, bins 1-21 are MiniBooNE and bins 22-42 are SciBooNE. The limits of the reconstructed antineutrino energy bins are shown in Table 6.2 and are exactly the same for MiniBooNE and SciBooNE.

The elements of the total error matrix are all positive. Since the total error matrix is a covariance matrix, the matrix is positive semi-definite and all its eigenvalues are nonnegative. A study of the total error matrix shows that its eigenvalues are all significantly larger than 0 making the total error matrix a positive definite matrix. Therefore, the total error matrix is invertible and has a Cholesky decomposition. Both properties are essential for the analysis. 
Flux Fractional Error Matrix RS

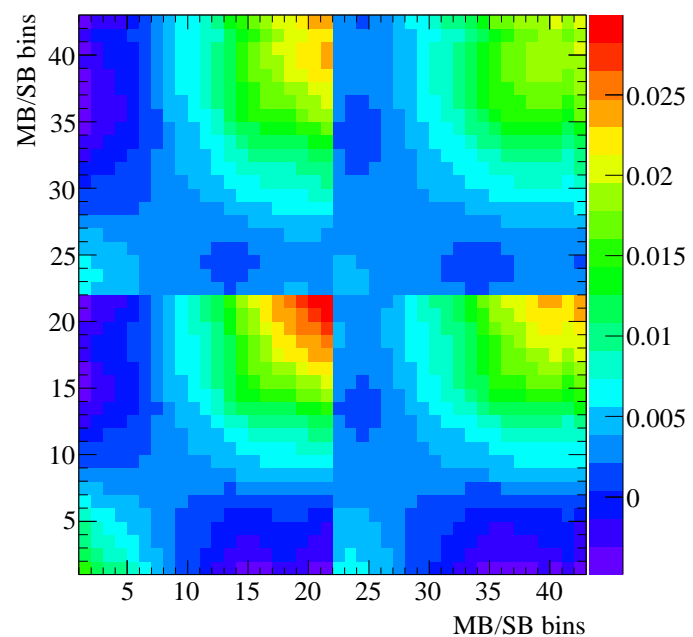

(a) Flux Right-Sign Fractional Error Matrix
Flux Fractional Error Matrix WS

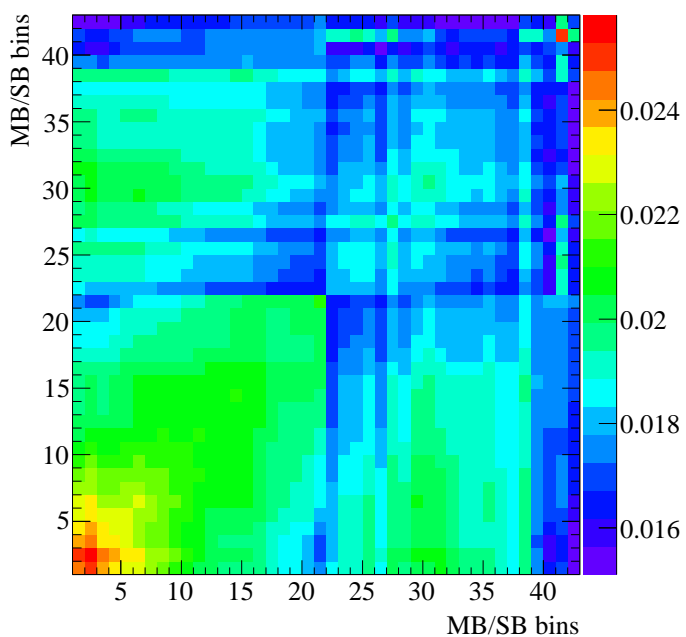

(b) Flux Wrong-Sign Fractional Error Matrix

Figure 6.6: The MiniBooNE-SciBooNE flux right-sign fractional error matrix (left) and flux wrong-sign fractional error matrix (right) are shown as a function of reconstructed antineutrino energy bins. Bins 1-21 are MiniBooNE and bins 22-42 are SciBooNE.

\subsection{Conclusion on Uncertainties}

In conclusion, all the relevant uncertainties from the neutrino (antineutrino) production to the neutrino (antineutrino) interactions in both detectors to the reconstruction and selection are addressed, described, and quantified. They can divided into three broad uncertainty categories: flux, neutrino (antineutrino) interaction, and detector. Figure 6.11 shows the $\sigma / \mu$ fractional uncertainties calculated from the square root of the diagonal elements of the error matrices as a function of reconstructed antineutrino energy in $\mathrm{GeV}$ for flux, neutrino interaction, detector, and total uncertainties in MiniBooNE. Figure 6.12 shows the $\sigma / \mu$ fractional uncertainties calculated from the square root of the diagonal elements of the error matrices as a function of reconstructed antineutrino energy in $\mathrm{GeV}$ for flux, neutrino interaction, detector, and total uncertainties in SciBooNE. From the figures, the neutrino interaction uncertainties are the largest source of uncertainties followed by detector uncertainties and flux uncertainties. Statistical uncertainties are very small. 

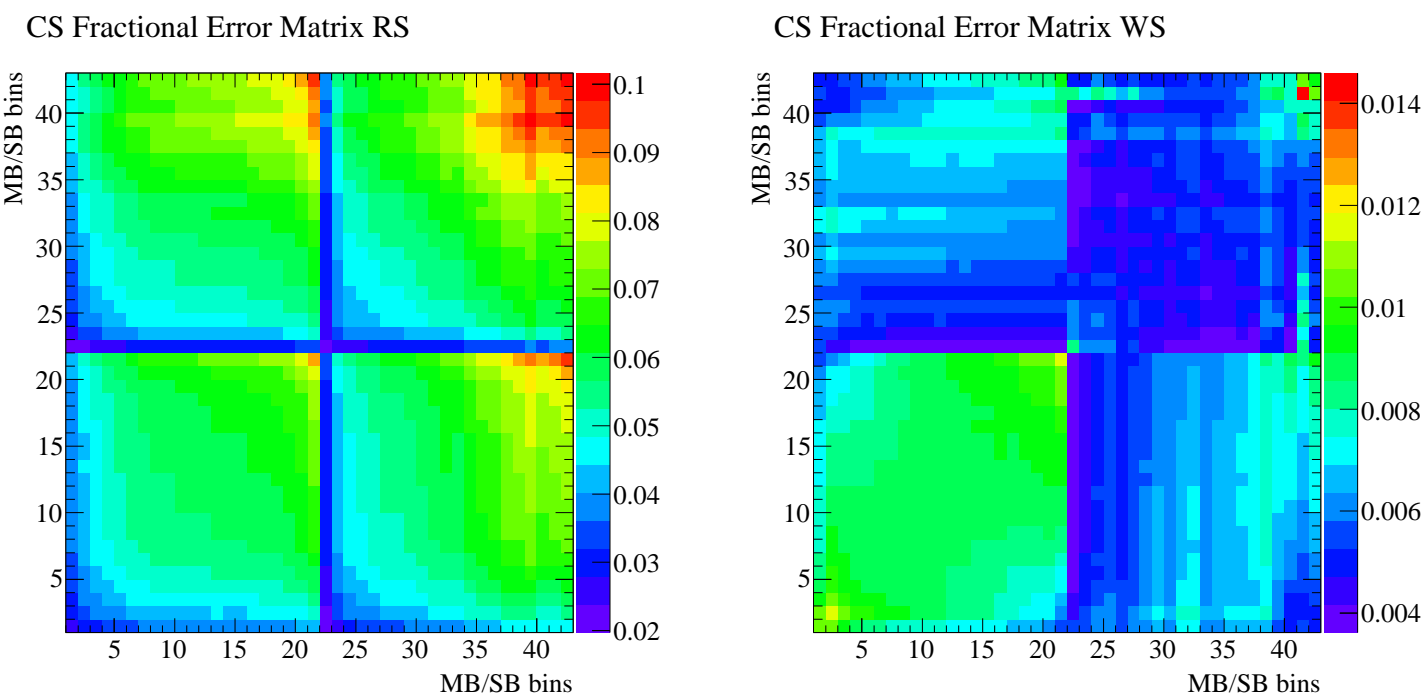

(a) Cross section Right-Sign Fractional Error Matrix (b) Cross section Wrong-Sign Fractional Error Matrix

Figure 6.7: The MiniBooNE-SciBooNE neutrino interaction right-sign fractional error matrix (left) and neutrino interaction wrong-sign fractional error matrix (right) are shown as a function of reconstructed antineutrino energy bins. Bins 1-21 are MiniBooNE and bins 22-42 are SciBooNE.

All the uncertainties are then expressed in the form of a total error matrix, a $42 \times$ 42 covariance matrix between reconstructed antineutrino energy bins of MiniBooNE and SciBooNE. The error matrix is constructed from fractional error matrices to allow the error matrix to vary as a function of the theory's oscillation parameters and provides the most accurate assessment of the uncertainties. Ultimately, the error matrix will provide a quantifiable measure of the differences between the reconstructed antineutrino energy distributions of data and MC predictions and be used directly in determining whether or not there are indications of antineutrino disappearance oscillations. 
SB Only Fractional Error Matrix RS

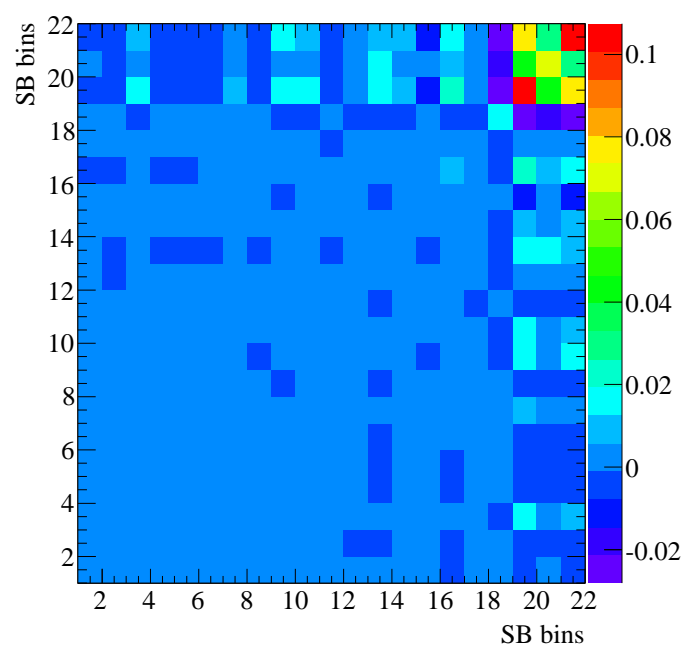

SB Only Fractional Error Matrix WS

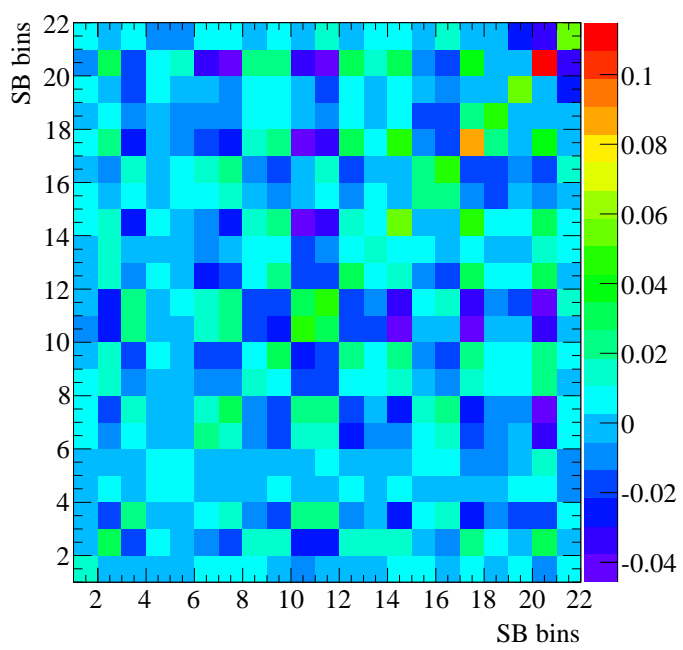

(a) SB Detector Right-Sign Fractional Error Matrix (b) SB Detector Wrong-Sign Fractional Error Matrix

Figure 6.8: The SciBooNE detector right-sign fractional error matrix (left) and detector wrong-sign fractional error matrix (right) are shown as a function of reconstructed antineutrino energy bins. The 21 SciBooNE antineutrino energy bins presented here have the exact same limits as the SciBooNE antineutrino energy bins in the MiniBooNE and SciBooNE error matrices for other uncertainties. 
MB Only Fractional Error Matrix RS

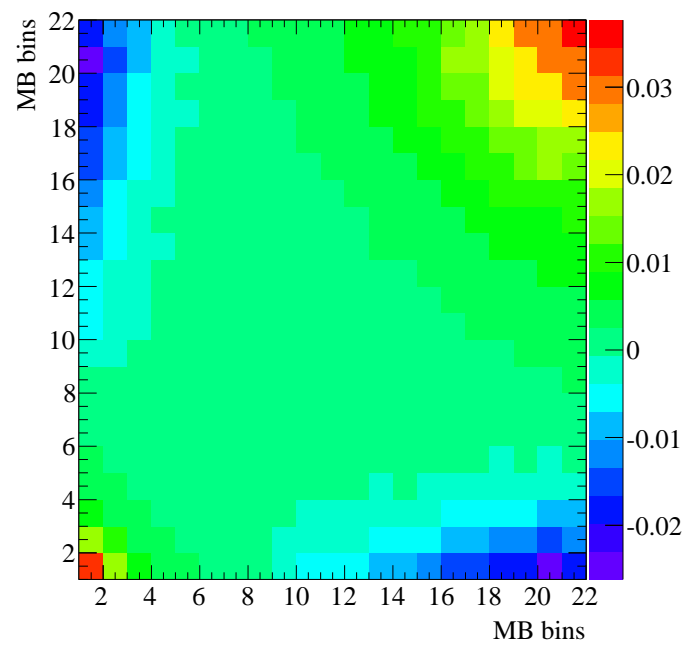

MB Only Fractional Error Matrix WS

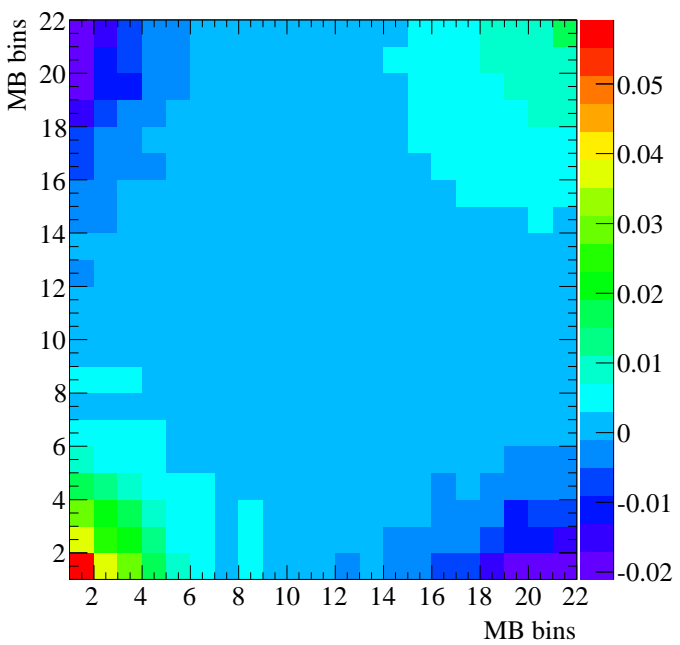

(a) MB Detector Right-Sign Fractional Error Matrix (b) MB Detector Wrong-Sign Fractional Error Matrix

Figure 6.9: The MiniBooNE detector right-sign fractional error matrix (left) and detector wrong-sign fractional error matrix (right) are shown as a function of reconstructed antineutrino energy bins. The 21 MiniBooNE antineutrino energy bins presented here have the exact same limits as the MiniBooNE antineutrino energy bins in the MiniBooNE and SciBooNE error matrices for other uncertainties. 


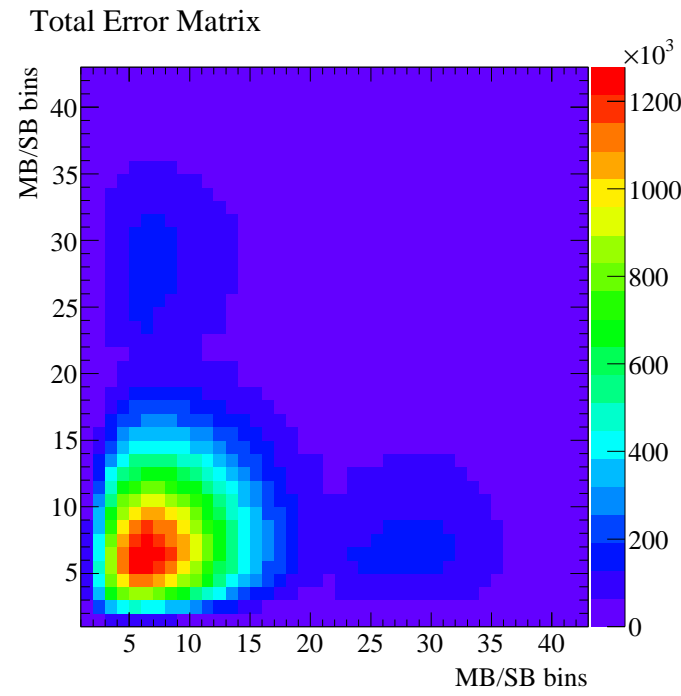

(a) Total Error Matrix
Total Correlation Error Matrix

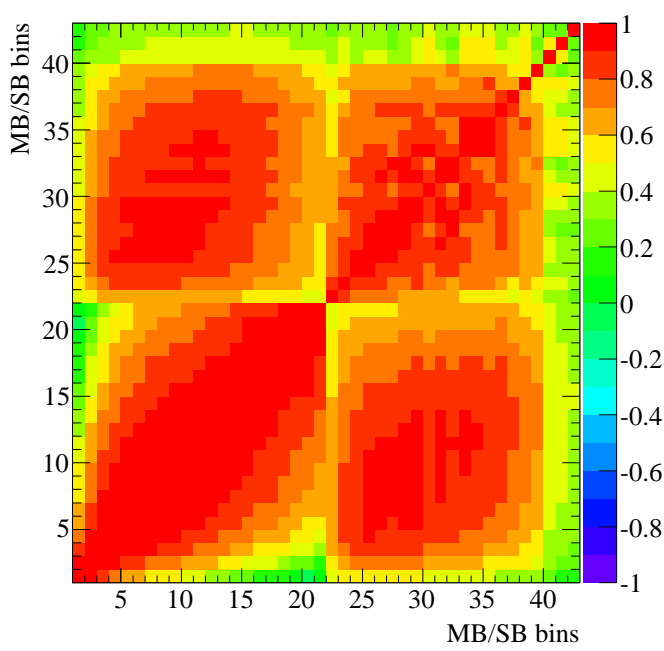

(b) Correlations of Total Error Matrix

Figure 6.10: The MiniBooNE-SciBooNE total error matrix (left) and its correlation (right) are shown as a function of reconstructed antineutrino energy bins. Bins 1-21 are MiniBooNE and bins 22-42 are SciBooNE. 


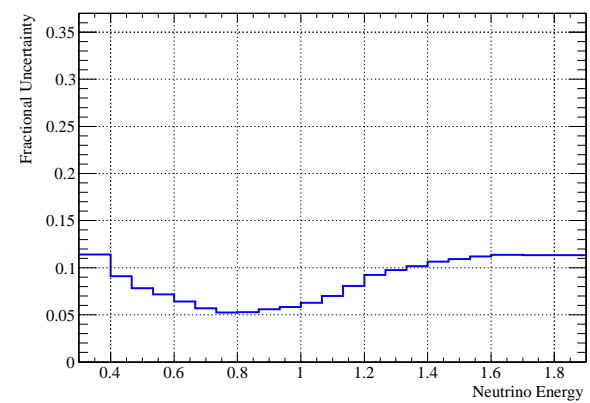

(a) MB Flux $\sigma / \mu$

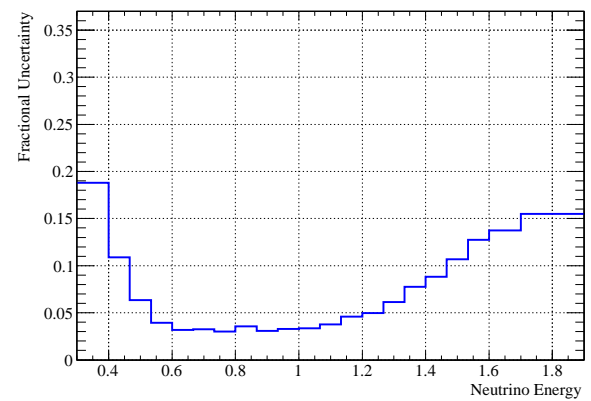

(c) MB Detector $\sigma / \mu$

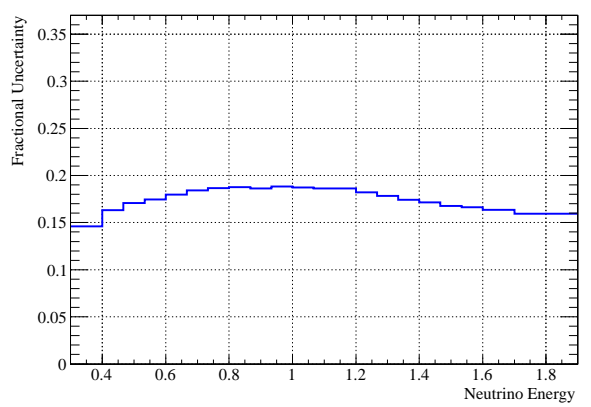

(b) MB Cross section $\sigma / \mu$

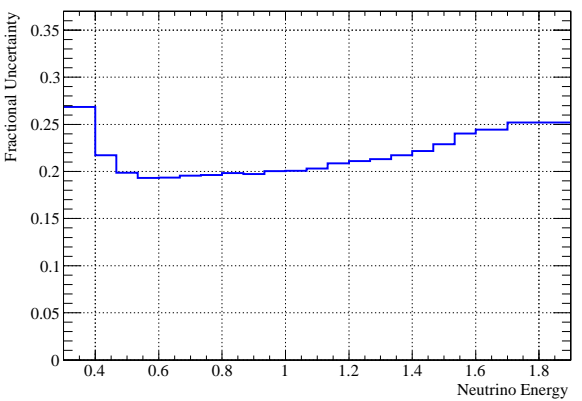

(d) MB Total $\sigma / \mu$

Figure 6.11: The MiniBooNE $\sigma / \mu$ fractional uncertainties for flux (top left), neutrino interactions (top right), detector (bottom left), and total systematic and statistical (bottom right) as a function of reconstructed antineutrino energy in $\mathrm{GeV}$. These are calculated from the diagonal elements of the error matrices so they do not capture any correlated error effects. 


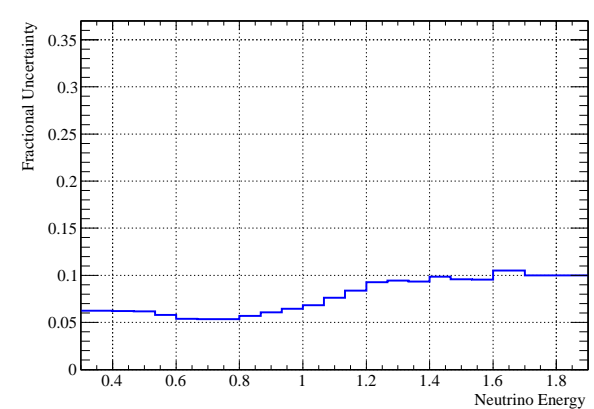

(a) SB Flux $\sigma / \mu$

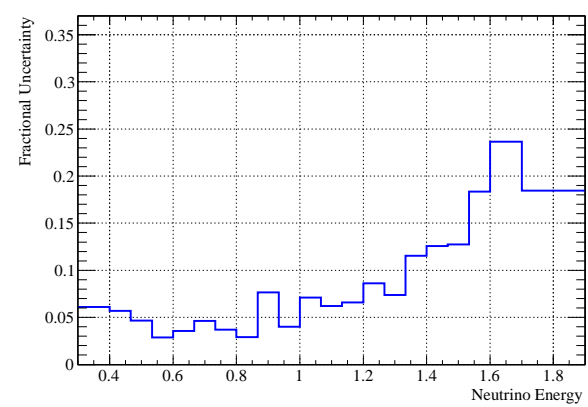

(c) $\mathrm{SB}$ Detector $\sigma / \mu$

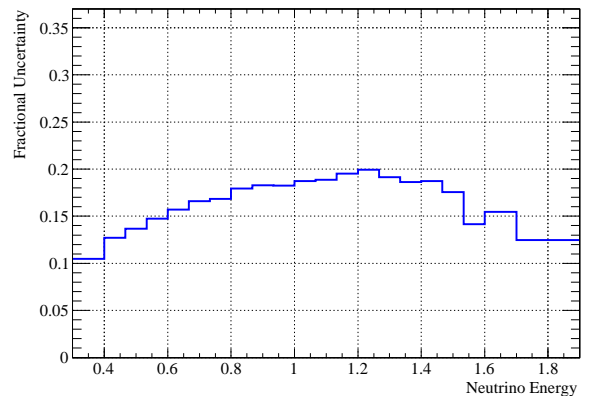

(b) SB Cross section $\sigma / \mu$

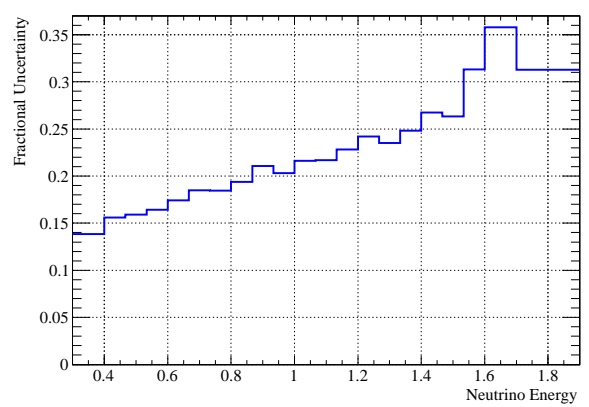

(d) SB Total $\sigma / \mu$

Figure 6.12: The SciBooNE $\sigma / \mu$ fractional uncertainties for flux (top left), neutrino interaction (top right), detector (bottom left), and total systematic and statistical (bottom right) as a function of reconstructed antineutrino energy in GeV. These are calculated from the diagonal elements of the error matrices so they do not capture any correlated error effects. 


\section{Chapter 7}

\section{Search for Dual Baseline Disappearance}

The purpose of this chapter is to state the muon antineutrino disappearance theory, explain and justify the methodology of the muon antineutrino disappearance analysis using the Feldman-Cousins method with a $\Delta \chi^{2}$ test statistic, and present the results. The antineutrino disappearance analysis is a "blind" analysis so all of the comprehensive studies of the robustness, consistency, and sensitivity of the analysis are done using MC predictions without any data. Data is unveiled at the last step and compared with MC predictions to determine the final analysis results.

The chapter is organized as follows. The muon antineutrino disappearance theory and formulation is introduced. The $\chi^{2}$ and the $\Delta \chi^{2}$ test statistic is then presented to explain how the antineutrino disappearance theory will be tested. The Feldman-Cousins method is presented to describe the statistical approach taken to extract the signals in the $\Delta \chi^{2}$ fit. Frequentist studies are then shown to test and understand the robustness, consistency, and sensitivity of the fit. Finally, data is unveiled and the chapter concludes with the results of the muon antineutrino disappearance analysis with comments. 


\subsection{Antineutrino Disappearance Oscillation Probability}

The muon antineutrino disappearance theory states that $\bar{\nu}_{\mu}$ oscillates over distance into a heavy sterile neutrino state $\bar{\nu}_{x}$. Since $\bar{\nu}_{x}$ is a sterile neutrino state, it does not interact through the weak interaction and cannot be detected. $\bar{\nu}_{\mu} \rightarrow \bar{\nu}_{x}$ oscillation will appear as a deficit in the number of $\bar{\nu}_{\mu}$ observed, referred to here as a "disappearance". Muon antineutrino disappearance is parameterized by a two-antineutrino oscillation model, where the oscillation survival probability for a $\bar{\nu}_{\mu}$ in the beam is given by

$$
P\left(\bar{\nu}_{\mu} \rightarrow \bar{\nu}_{x}\right)=1-\sin ^{2} 2 \theta \sin ^{2} \frac{1.27 \Delta m^{2} L}{E} .
$$

where $L$ is the path length in kilometers, $E$ is the antineutrino energy in $\mathrm{GeV}, \theta$ is the mixing angle, and $\Delta m^{2}$ is the difference in the squares of the masses of two different mass eigenstates.

If a detector is placed at an appropriate fixed distance away from the $\bar{\nu}_{\mu}$ production source, and if muon antineutrino disappearance exists, the $\bar{\nu}_{\mu} \rightarrow \bar{\nu}_{x}$ oscillation will produce a distinct signature in antineutrino energy, since antineutrinos with different energies oscillate with different probabilities for the same distance traveled. For this reason, the reconstructed antineutrino energy distribution is the distribution used for comparing the data and MC predictions along with the error matrix in the $\Delta \chi^{2}$ fit described in Section 7.2 . $\mathrm{MC}$ simulation is used to determine the muon antineutrino production in the decay tunnel. Data from the MiniBooNE and SciBooNE detector, which are at two different fixed distances away from the muon antineutrino production source, will be used to confirm or deny whether muon antineutrino disappearance exists for the $\sin ^{2} 2 \theta$ and $\Delta m^{2}$ parameter space by comparing to various MC predictions based on different $\bar{\nu}_{\mu}$ survival probabilities from different $\sin ^{2} 2 \theta$ and $\Delta m^{2}$ values.

This analysis is a test on the theory of $\bar{\nu}_{\mu}$ disappearance so the number of $\bar{\nu}_{\mu}$ events must be allowed to vary based on the oscillation parameters $\sin ^{2} 2 \theta$ and $\Delta m^{2}$ of the theory. Though CPT invariance requires that $\bar{\nu}_{\mu}$ disappearance demands $\nu_{\mu}$ disappearance, the number of $\nu_{\mu}$ events is fixed in this analysis since $\nu_{\mu}$ disappearance has not been observed based on previous measurement 37. Very few $\nu_{e}$ and $\bar{\nu}_{e}$ events pass the MiniBooNE and SciBooNE selection criteria so their uncertainties relative to $\nu_{\mu}$ and $\bar{\nu}_{\mu}$ events are very 
small. $\nu_{e}$ and $\bar{\nu}_{e}$ disappearance and appearance are also not in the scope of this analysis. Therefore, the number of $\nu_{e}$ and $\bar{\nu}_{e}$ events are fixed.

Previous muon antineutrino disappearance measurements from MiniBooNE in 2009 [38. and the CCFR experiment [40] observed no $\bar{\nu}_{\mu}$ disappearance and set a lower limit on the value of $\Delta m^{2}$. Based on these measurements, the the upper limit of $\Delta m^{2}$ for the analysis is set at $\Delta m^{2}=100 \mathrm{eV}^{2}$. Due to limits from the data statistics, the location of the SciBooNE and MiniBooNE detectors relative to the $\bar{\nu}_{\mu}$ source, and the uncertainties involved in the analysis, frequentist studies described in Section 7.4 show that the lower limit of the $\Delta m^{2}$ for the analysis should be set at $\Delta m^{2}=0.01 \mathrm{eV}^{2}$. Therefore, the parameter space being tested in this analysis is a two dimensional parameter space of $\Delta m^{2}$ and $\sin ^{2} 2 \theta$ with limits $100-0.01 \mathrm{eV}^{2}$ and $0-1$, respectively.

\section{$7.2 \quad \chi^{2}$ and $\Delta \chi^{2}$ Test Statistic}

The $\chi^{2}$ statistic is

$$
\chi^{2}=\sum_{i, j=1}^{42}\left(D_{i}-N_{i}\right)\left(M^{-1}\right)_{i j}\left(D_{j}-N_{j}\right),
$$

where $\left(M^{-1}\right)_{i j}$ is the $i j$-th element of the inverse of the error matrix $M$, the covariance matrix in MiniBooNE and SciBooNE reconstructed antineutrino energy $\left(E_{\nu}^{Q E}\right)$ bins described in Equation 6.7 and presented in Section 6.6. $D_{i}\left(D_{j}\right)$ is the data count in bin $i(j)$ and $N_{i}$ $\left(N_{j}\right)$ is the MC prediction for bin $i(j)$, in MiniBooNE and SciBooNE $E_{\nu}^{Q E}$ bins. $N_{i}$ is the sum of neutrino (WS) and antineutrino (RS) events in the $i$-th bin:

$$
N_{i}=N_{i}^{R S}\left(\Delta m^{2}, \sin ^{2} 2 \theta\right)+N_{i}^{W S}
$$

$N_{j}$ is similarly defined as $N_{i}$. As shown in Equation 7.3 and explained in Section 7.1, only the number of predicted RS antineutrino events depend on the oscillation parameters, $\Delta m^{2}$ and $\sin ^{2} 2 \theta$, in this two-antineutrino $\bar{\nu}_{\mu}$ oscillation model and the number of predicted WS neutrino events are fixed. The index $i$ runs from 1 to 42 with the first 21 bins being the MiniBooNE $E_{\nu}^{Q E}$ bins ordered in ascending energy and the second 21 bins being SciBooNE $E_{\nu}^{Q E}$ bins ordered in ascending energy. The limits of the $E_{\nu}^{Q E}$ bins for index $i$ are listed in Table 6.2. 
For the goodness of fit test to determine whether the data agrees with the MC prediction, a $\Delta \chi^{2}$ test statistic is used. It is defined as:

$$
\Delta \chi^{2}=\chi^{2}\left(N\left(\Delta m^{2}, \sin ^{2} 2 \theta\right), M\left(\Delta m^{2}, \sin ^{2} 2 \theta\right)\right)-\chi^{2}\left(N\left(\theta_{\mathrm{BF}}\right), M\left(\theta_{\mathrm{BF}}\right)\right)
$$

where $\Delta m^{2}$ and $\sin ^{2} 2 \theta$ are the oscillation parameters at a given test point and $\theta_{\mathrm{BF}}$ refers to the oscillation parameters $\Delta m^{2}$ and $\sin ^{2} 2 \theta$ at the best fit point in the entire $\Delta m^{2}, \sin ^{2} 2 \theta$ parameter space region $\left(\Delta m^{2}\right.$ from 0.01 to $100 \mathrm{eV}^{2} . N$ and $M$ are the MC prediction and error matrix, respectively, and hence, are a function of the oscillation parameters.

In general, since the fit has two parameters $\Delta m^{2}$ and $\sin ^{2} 2 \theta$, the $\Delta \chi^{2}$ at each point in $\Delta m^{2}, \sin ^{2} 2 \theta$ parameter space should follow a $\chi^{2}$ distribution with two degrees of freedom (DOF), which corresponds to a $90 \%$ confidence level (CL) value for goodness of fit of less than 4.61. But previous studies done to measure $\nu_{\mu}$ and $\bar{\nu}_{\mu}$ disappearance using the $\Delta \chi^{2}$ test statistic and frequentist studies done for this analysis (described in Section 7.4) show that this is not exactly the case, though the $\chi^{2}$ distribution with two DOF can be used as an approximation. The reason is due to the fact that the best fit point $\theta_{\mathrm{BF}}$ doesn't exactly correspond to the true best fit point since fluctuations can cause the $\theta_{\mathrm{BF}}$ to deviate from the true best fit point. For example, assume that there is no $\bar{\nu}_{\mu}$ disappearance. Then, the true best fit point would be the line with $\sin ^{2} 2 \theta$ at 0 (there is also no $\Delta m^{2}$ dependence and the $\chi^{2}$ distribution reduces to one DOF). But because of statistical uncertainties in the data and systematic uncertainties in creating the MC prediction, the data and MC prediction might not exactly agree due to these small fluctuations. The small fluctuations can mimic a small oscillation signal and create a best fit point $\theta_{\mathrm{BF}}$ with a non-zero $\sin ^{2} 2 \theta$. The $\left(\chi^{2}\right)$ $\Delta \chi^{2}$ value at $\theta_{\mathrm{BF}}$ will be slightly smaller than the $\left(\chi^{2}\right) \Delta \chi^{2}$ value at the true best fit point. To address these challenges, the Feldman-Cousins method described in Section 7.3 is used to determine the confidence level of the data relative to the MC prediction at each point in $\Delta m^{2}, \sin ^{2} 2 \theta$ space.

\subsection{Feldman-Cousins Method}

The method of Feldman and Cousins 113] is used to determine the significance of the $\Delta \chi^{2}$ at each point $\Delta m^{2}, \sin ^{2} 2 \theta$ in parameter space that corresponds to a certain confidence 
level of acceptance or rejection. To obtain the $90 \%$ confidence level exclusion region for $\bar{\nu}_{\mu}$ disappearance, a smooth $\Delta \chi^{2}$ distribution is formed for each point $\Delta m^{2}, \sin ^{2} 2 \theta$ in parameter space using hundreds of iterations of generated fake data (described in Section 7.4) at that $\Delta m^{2}, \sin ^{2} 2 \theta$. The fake data $\Delta \chi^{2}$ distribution changes for each $\Delta m^{2}, \sin ^{2} 2 \theta$ point and accounts for the shifts in the $\Delta \chi^{2}$ due to $\theta_{\mathrm{BF}}$ not corresponding exactly to the true best fit point as mentioned in Section 7.2. The $\Delta \chi^{2}$ value from actual data at each $\Delta m^{2}, \sin ^{2} 2 \theta$ is then compared to the fake data $\Delta \chi^{2}$ distribution at each $\Delta m^{2}, \sin ^{2} 2 \theta$. If the $\Delta \chi^{2}$ value from actual data is larger than $90 \%$ of the all the fake data $\Delta \chi^{2}$ values at a $\Delta m^{2}, \sin ^{2} 2 \theta$ point, then the $\Delta m^{2}, \sin ^{2} 2 \theta$ point in parameter space is excluded at $90 \%$ confidence level. The aggregation of all the excluded $90 \%$ confidence level $\Delta m^{2}, \sin ^{2} 2 \theta$ points forms the $90 \%$ confidence level exclusion region. The region not excluded by the $90 \%$ confidence level exclusion region would be a basis for the detection of a $\bar{\nu}_{\mu}$ oscillation signal or is a region beyond the sensitivity of the analysis.

For the analysis, the oscillation parameter space is divided into a grid of points with $\sin ^{2} 2 \theta$ divided into 200 linear increments from 0 to 1 and $\Delta m^{2}$ divided into 300 logarithmic increments from -2 to 2 , which are the logarithmic limits of $0.01-100 \mathrm{eV}^{2}$. Including the boundaries of the $\Delta m^{2}, \sin ^{2} 2 \theta$ parameter space, the analysis uses a total of 60501 oscillation parameter points to determine the signal or exclusion region for $\bar{\nu}_{\mu}$ disappearance. However, creating a fake data $\Delta m^{2}$ value at each oscillation parameter point is computationally intensive due to the necessity of calculating $\chi^{2}$ at each oscillation parameter point in order to find $\chi^{2}\left(N\left(\theta_{\mathrm{BF}}\right), M\left(\theta_{\mathrm{BF}}\right)\right)$ in the entire parameter space for each fake data $\Delta m^{2}$ value. To accomplish this, a courser, subset grid of points is used for the Feldman-Cousins $\Delta \chi^{2}$ distribution. The Feldman-Cousins $\Delta \chi^{2}$ grid divides the oscillation parameter space into 40 linear increments from 0 to 1 for $\sin ^{2} 2 \theta$ and 60 logarithmic increments from -2 to 2 for $\Delta m^{2}$ for a total of 2501 points (the Feldman-Cousins grid uses every fifth grid point for the analysis oscillation parameter space grid). At least 500 fake data $\Delta \chi^{2}$ values at generated each point in order for the fake data $\Delta \chi^{2}$ distribution to be smooth and the confidence level of acceptance or rejection with data to be accurate. More fake data $\Delta \chi^{2}$ values (on the order of 1000) are generated in the region of the oscillation parameter space most likely to produce a $\bar{\nu}_{\mu}$ signal or limit for exclusion. At each of the Feldman-Cousins grid points, 
a cutoff value for a certain confidence level of acceptance or rejection (e.g. confidence level for $90 \%$ exclusion of oscillation signal) is determined from its corresponding fake data $\Delta \chi^{2}$ distribution. This confidence level cutoff value is then linearly interpolated between the Feldman-Cousins grid points to all of the analysis's 60501 parameter space grid points so that each of the analysis's 60501 parameter space points has a unique value for acceptance or rejection. Data is finally unveiled and the $\Delta \chi^{2}$ is calculated at each analysis parameter space grid point and compared to the confidence level cutoff value to determine acceptance or rejection to produce a signal or exclusion limit.

\subsection{Frequentist Studies}

In order to better understand the robustness, consistency, and sensitivity of the $\Delta \chi^{2}$ fit in the parameter space without using the data, fake data must be used. Given the assumption that the MC predictions are an accurate reflection of actual data and the uncertainties involved are fairly well understood, fake data can be generated using the MC prediction and the combined error matrix.

To create the fake data, first, a Cholesky decomposition is performed on the combined error matrix $M$ :

$$
M\left(\Delta m^{2}, \sin ^{2} 2 \theta\right)=L L^{*}
$$

where $L$ is a lower triangular matrix and $L^{*}$ is the conjugate transpose of $L$. A 42 dimensional vector $u$ is created, where each of the 42 elements represents one of the reconstructed antineutrino energy bins of MiniBooNE and SciBooNE. Each element of $u$ are independently drawn from a Gaussian distribution with mean 0 and variance 1. A fluctuated fake data histogram, $N_{\text {fake }}$, is given by

$$
N_{\text {fake }}=N_{\text {default }}\left(\Delta m^{2}, \sin ^{2} 2 \theta\right)+L u
$$

where $N_{\text {default }}$ is the default MC prediction assuming an oscillation signal with oscillation parameters at point $\Delta m^{2}, \sin ^{2} 2 \theta$. The term $L u$ can be understood as the correlated systematic and statistical uncertainties added to the default MC prediction at that $\Delta m^{2}$, $\sin ^{2} 2 \theta$ point. Note that since $M$, the combined error matrix, is a function of the oscillation 
parameters, $L$ is not constant across the parameter space and the $L u$ values can vary significantly (and not only through $u$ ) across the parameter space. This method of fake data generation efficiently allows hundreds of fake data to be generated at each $\Delta m^{2}$, $\sin ^{2} 2 \theta$ point with minimal computing time for use in the Feldman-Cousins methodology.

Figures 7.1 and 7.2 show that the fake data are being generated properly. Figure 7.1 shows the normalization variations between MiniBooNE and SciBooNE in a scatter plot. Each point in the two dimensional scatter plot represents a MiniBooNE and SciBooNE fake data normalization (both normalized relative to default MC prediction). As expected, the uncertainties that cause the MiniBooNE and SciBooNE MC predictions to change in normalization have the same underlying sources (e.g. antineutrino cross section) so normalization is correlated and therefore, scatter points lie along the $y=x$ diagonal line. Figure 7.2 shows the SciBooNE fake data value normalized to default SciBooNE prediction over MiniBooNE fake data value normalized to default MiniBooNE prediction as a function of reconstructed antineutrino energy bins. As expected, the histograms cluster around the value of 1 with larger fluctuations at the lower and higher antineutrino energy bins, which corresponds to the higher systematic and statistical uncertainties in the analysis at lower and higher antineutrino energy.

The robustness, consistency, and sensitivity of the $\Delta \chi^{2}$ fit can now be tested. To make things simpler, the $\Delta \chi^{2}$ distribution at each $\Delta m^{2}, \sin ^{2} 2 \theta$ parameter space point is approximated using a $\chi^{2}$ distribution with 2 DOF $\left(\sin ^{2} 2 \theta\right.$ and $\left.\Delta m^{2}\right)$ instead of using the computing-intensive Feldman-Cousins method. The $90 \%$ and $3-\sigma$ confidence levels of oscillation signal exclusion are made at values of 4.61 and 11.38, respectively, corresponding to $90 \%$ and $3-\sigma$ confidence levels of a $\chi^{2}$ distribution with 2 DOF. This allows the $\Delta \chi^{2}$ fit to be efficiently tested without loss of generality, though slight deviations between exclusion limits and oscillation signal regions calculated using the $\chi^{2}$ distribution with 2 DOF approximation and the Feldman-Cousins method might occur at low $\Delta m^{2}$ and low $\sin ^{2} 2 \theta$ of the parameter space.

Figure 7.3 shows the averaged exclusion limits at the $90 \%$ and $3-\sigma$ confidence levels and possible oscillation signal region and best fit $\Delta \chi^{2}$ points for many fake data sets all with no oscillation signal assumed. The excluded region is the region with high $\Delta m^{2}(\log$ 


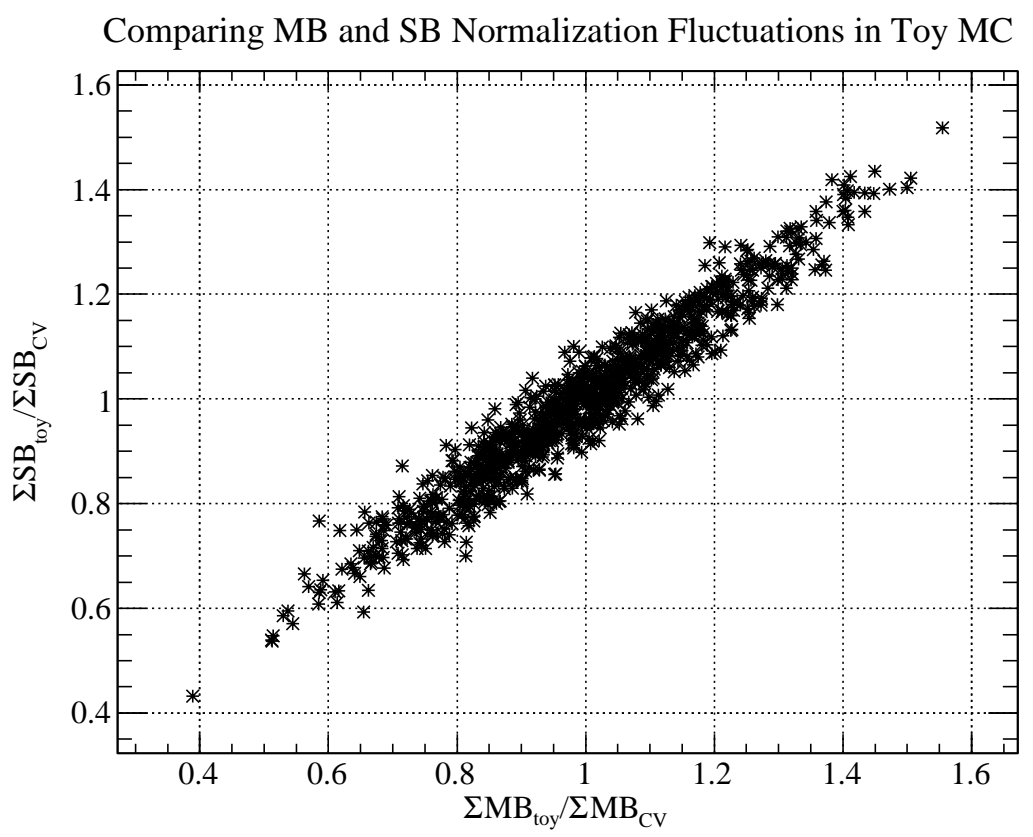

Figure 7.1: Two dimensional scatter plot of normalization variations of MiniBooNE and SciBooNE. Each point in the scatter plot represents a MiniBooNE and SciBooNE fake data normalization (both normalized relative to default MC prediction). The $\mathrm{x}$-axis is the MiniBooNE normalization (sum of all MB reconstructed antineutrino energy bins for one fake data relative to sum of all default MB reconstructed antineutrino energy bins) and the $\mathrm{y}$-axis is the SciBooNE normalization (sum of all SB reconstructed antineutrino energy bins for one fake data relative to sum of all default SB reconstructed antineutrino energy bins). 100 scatter points are plotted and no oscillation signal is assumed.

$\left.\Delta m^{2}>-0.5\right)$ and high $\sin ^{2} 2 \theta\left(\sin ^{2} 2 \theta>0.1\right)$ where the test can uniformly rule out a large region of oscillation parameter space, which is what is desired and expected. The figure also shows the overall sensitivity allowed by the analysis. The allowed oscillation signal region (region not excluded at the $90 \%$ confidence level) is in light blue and at low $\Delta m^{2}$ (log $\left.\Delta m^{2}<-0.5\right)$ and low $\sin ^{2} 2 \theta\left(\sin ^{2} 2 \theta<0.1\right)$, the region where either the magnitude of the disappearance oscillation (controlled by $\sin ^{2} 2 \theta$ ) is so small that it is beyond the sensitivity within uncertainties of the analysis (very small $\sin ^{2} 2 \theta$ value) or the oscillation is so long relative to energies of the $\bar{\nu}_{\mu}$ being measured that the $\bar{\nu}_{\mu}$ have not yet had the distance to 


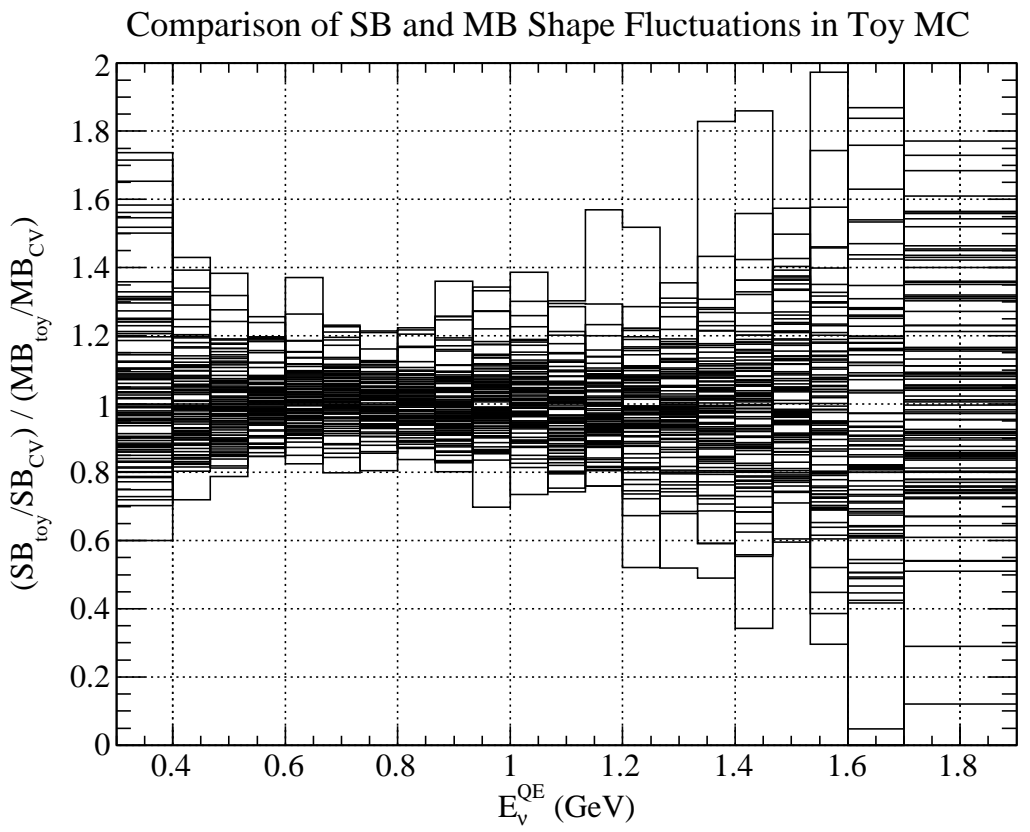

Figure 7.2: SciBooNE fake data value over MiniBooNE fake data value as a function of reconstructed antineutrino energy bins. Both the SciBooNE and MiniBooNE fake data values in each bin are normalized to default SciBooNE and MiniBooNE MC prediction bin values, respectively. 100 histograms are plotted and no oscillation signal is assumed.

oscillate into its sterile $\bar{\nu}_{x}$ partner before reaching the MiniBooNE detector. The best fit points for the fake data sets cluster around $\log \Delta m^{2}=1$ and $\sin ^{2} 2 \theta=0.075$, instead of at $\sin ^{2} 2 \theta=0$, due to the fact that the fake data fluctuations can mimic small oscillation signals (as described in Section 7.2). Figure 7.4 shows fake data $\Delta \chi^{2}$ and $\chi^{2}$ distribution assuming no oscillation signal at the no oscillation signal line. The fake data $\Delta \chi^{2}$ and $\chi^{2}$ distributions fit the $\chi^{2}$ distributions quite well assuming 2 DOF and 40 DOF, respectively. This confirms the assumption that the fake data $\Delta \chi^{2}$ distribution follows a $\chi^{2}$ distribution with 2 DOF so the $\chi^{2}$ distribution with 2 DOF can be an approximate substitute for the Feldman-Cousins $\Delta \chi^{2}$ distributions. Also the fake data $\chi^{2}$ distribution is exactly what is expected since there are a total of $42 \mathrm{MiniBooNE}$ and SciBooNE reconstructed antineutrino energy bins corresponding to 42 DOF minus the 2 DOF from the oscillation parameter fit.

Figure 7.5 shows the averaged exclusion limits at the $90 \%$ and $3-\sigma$ confidence levels and 


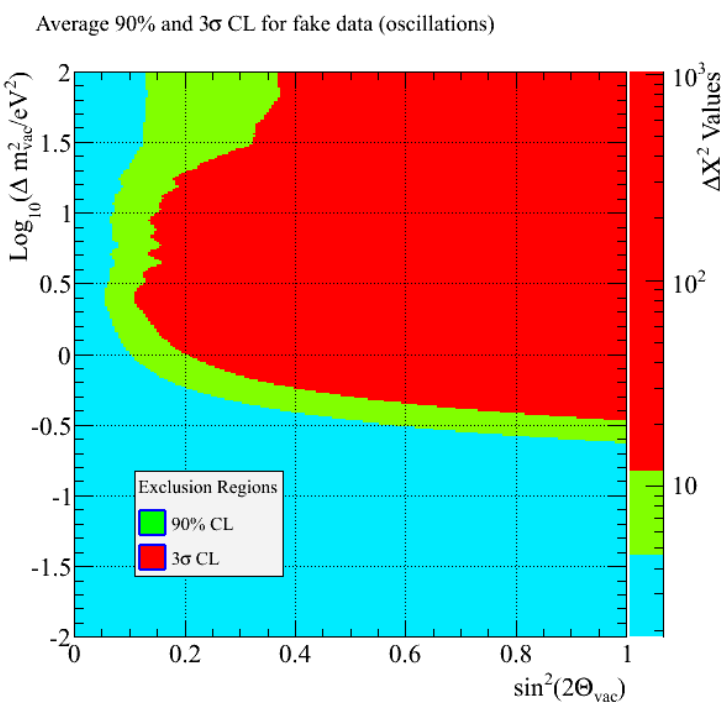

(a) Exclusion Limit From Fake Data Sets Assuming No Oscillation Signal best fit points

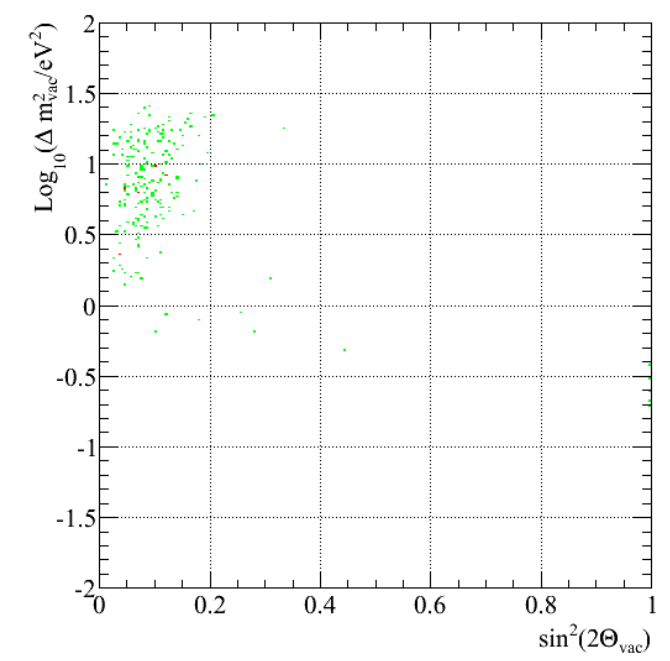

(b) Best Fit $\Delta \chi^{2}$ Points From Fake Data Sets

Figure 7.3: Averaged exclusion limits at the $90 \%$ and $3-\sigma$ confidence levels and possible oscillation signal region (left) and best fit $\Delta \chi^{2}$ points (right) for many fake data sets all with no oscillation signal assumed. The allowed oscillation signal region is in light blue. The regions were produced assuming the $\Delta \chi^{2}$ distribution is a $\chi^{2}$ distribution with 2 DOF.

possible oscillation signal region and best fit $\Delta \chi^{2}$ points for many fake data sets assuming an oscillation signal of $\Delta m^{2}=1.0 \mathrm{eV}^{2}$ and $\sin ^{2} 2 \theta=0.2$. The allowed oscillation signal region (region not excluded at the $90 \%$ confidence level) is a light blue band that clearly includes the oscillation signal point. The best fit points for the fake data sets cluster around $\Delta m^{2}=1.0 \mathrm{eV}^{2}$ and $\sin ^{2} 2 \theta=0.2$ along the oscillation signal band. Figure 7.6 shows fake data $\Delta \chi^{2}$ and $\chi^{2}$ distribution assuming oscillation signal of $\Delta m^{2}=1.0 \mathrm{eV}^{2}$ and $\sin ^{2} 2 \theta=0.2$ at that oscillation parameter point. The fake data $\Delta \chi^{2}$ and $\chi^{2}$ distributions fit the $\chi^{2}$ distributions quite well assuming 2 DOF and 40 DOF, respectively. Therefore, the fake data $\Delta \chi^{2}$ distribution follows a $\chi^{2}$ distribution with 2 DOF and the $\chi^{2}$ distribution is consistent with the 42 MiniBooNE and SciBooNE antineutrino energy bins minus the two DOF from the oscillation parameters. The test demonstrates how a signal at or near $\Delta m^{2}=1.0 \mathrm{eV}^{2}$ and $\sin ^{2} 2 \theta=0.2$ would be distinguished and found if it existed in the data. 


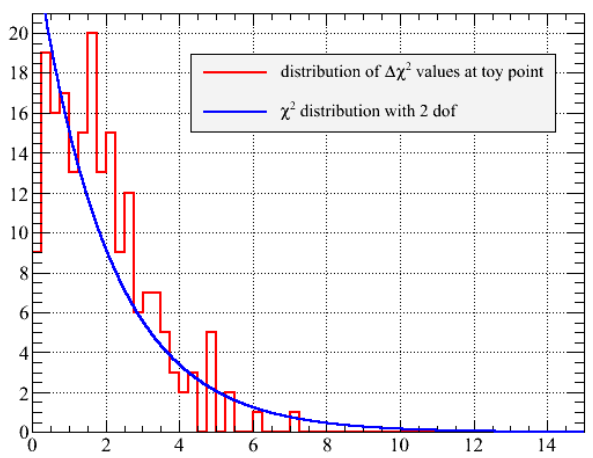

(a) Fake Data $\Delta \chi^{2}$ Values

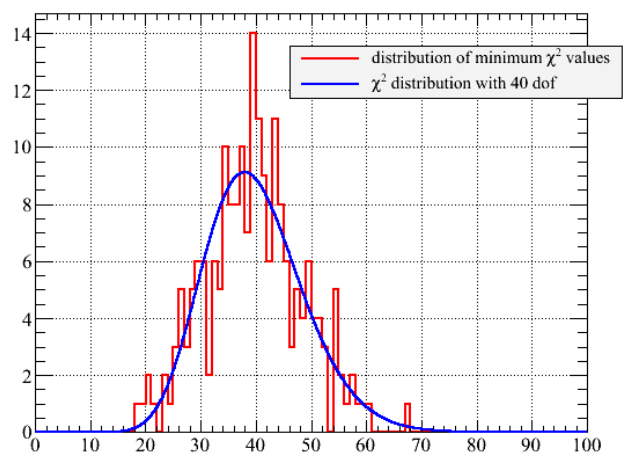

(b) Fake Data $\chi^{2}$ Values

Figure 7.4: Fake data $\Delta \chi^{2}$ distribution with the blue line showing a $\chi^{2}$ distribution with $2 \mathrm{DOF}$ (left) and fake data $\chi^{2}$ distribution with the blue line showing a $\chi^{2}$ distribution with 40 DOF right for many fake data sets and MC prediction all with no oscillation signal assumed.

Figure 7.7 shows the averaged exclusion limits at the $90 \%$ and $3-\sigma$ confidence levels and possible oscillation signal region and best fit $\Delta \chi^{2}$ points for many fake data sets assuming an oscillation signal of $\Delta m^{2}=1.0 \mathrm{eV}^{2}$ and $\sin ^{2} 2 \theta=0.4$. The allowed oscillation signal region (region not excluded at the $90 \%$ confidence level) is a light blue ellipse that clearly includes the oscillation signal point. The best fit points for the fake data sets cluster around $\Delta m^{2}=1.0 \mathrm{eV}^{2}$ and $\sin ^{2} 2 \theta=0.4$ along the oscillation signal ellipse. Figure 7.8 shows fake data $\Delta \chi^{2}$ and $\chi^{2}$ distribution assuming oscillation signal of $\Delta m^{2}=1.0 \mathrm{eV}^{2}$ and $\sin ^{2} 2 \theta=0.4$ at that oscillation parameter point. The fake data $\Delta \chi^{2}$ and $\chi^{2}$ distributions fit the $\chi^{2}$ distributions assuming 2 DOF and 40 DOF, respectively, well. Therefore, the fake data $\Delta \chi^{2}$ distribution follows a $\chi^{2}$ distribution with 2 DOF and the $\chi^{2}$ distribution is consistent with the 42 MiniBooNE and SciBooNE antineutrino energy bins minus the two DOF from the oscillation parameters. The test demonstrates how a signal at or near $\Delta m^{2}=1.0 \mathrm{eV}^{2}$ and $\sin ^{2} 2 \theta=0.4$ would be distinguished and found if it existed in the data.

Figure 7.9 shows the averaged exclusion limits at the $90 \%$ and $3-\sigma$ confidence levels and possible oscillation signal region and best fit $\Delta \chi^{2}$ points for many fake data sets assuming an oscillation signal of $\Delta m^{2}=10.0 \mathrm{eV}^{2}$ and $\sin ^{2} 2 \theta=0.2$. The allowed oscillation signal region 


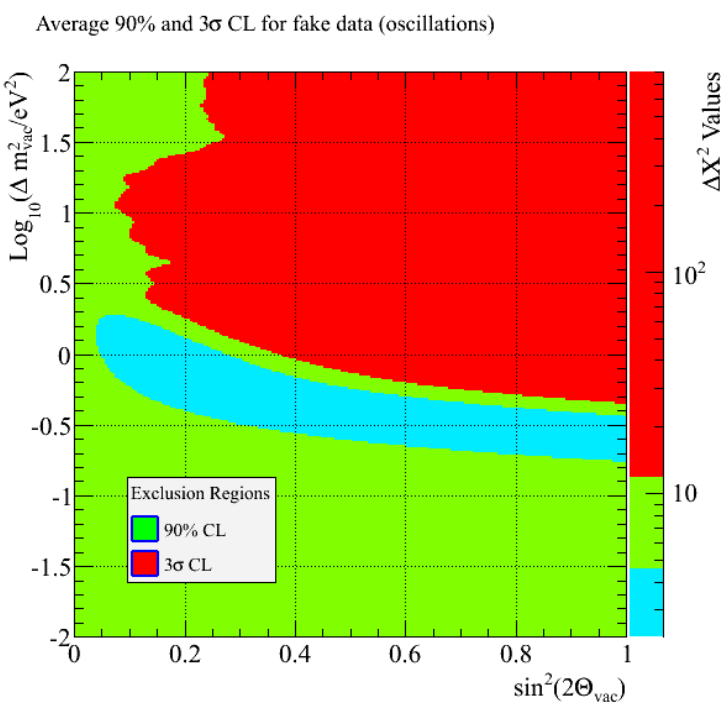

(a) Exclusion Limit From Fake Data Sets Assuming Oscillation Signal With $\Delta m^{2}=1.0 \mathrm{eV}^{2}$ and $\sin ^{2} 2 \theta=$ best fit points

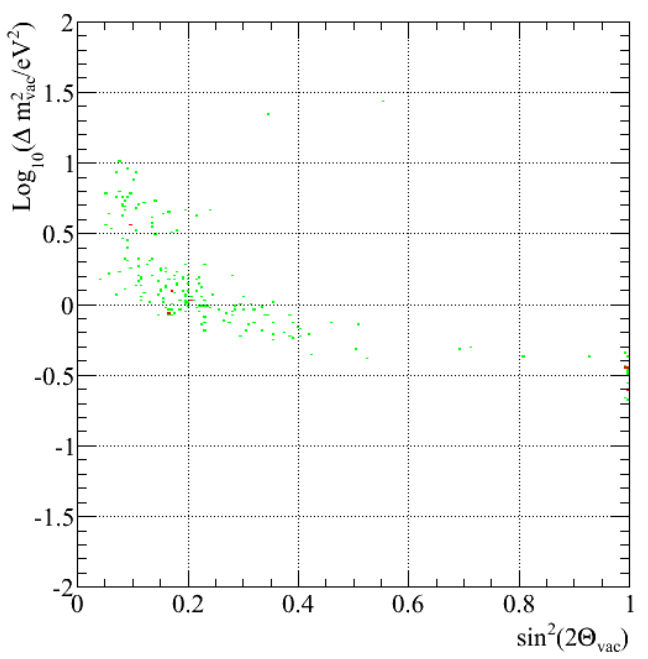

(b) Best Fit $\Delta \chi^{2}$ Points From Fake Data Sets 0.2

Figure 7.5: Averaged oscillation signal region and exclusion limits at the $90 \%$ and $3-\sigma$ confidence levels (left) and best fit $\Delta \chi^{2}$ points (right) for many fake data sets all with oscillation signal of $\Delta m^{2}=1.0 \mathrm{eV}^{2}$ and $\sin ^{2} 2 \theta=0.2$ assumed. The allowed oscillation signal region is in light blue. The regions were produced assuming the $\Delta \chi^{2}$ distribution is a $\chi^{2}$ distribution with 2 DOF.

(region not excluded at the $90 \%$ confidence level) is a tiny light blue ellipse that basically only includes the oscillation signal point. The best fit points for the fake data sets cluster around $\Delta m^{2}=10.0 \mathrm{eV}^{2}$ and $\sin ^{2} 2 \theta=0.2$ along the oscillation signal ellipse. Figure 7.10 shows fake data $\Delta \chi^{2}$ and $\chi^{2}$ distribution assuming oscillation signal of $\Delta m^{2}=10.0 \mathrm{eV}^{2}$ and $\sin ^{2} 2 \theta=0.2$ at that oscillation parameter point. The fake data $\Delta \chi^{2}$ and $\chi^{2}$ distributions fit the $\chi^{2}$ distributions well assuming 2 DOF and 40 DOF, respectively. Therefore, the fake data $\Delta \chi^{2}$ distribution follows a $\chi^{2}$ distribution with $2 \mathrm{DOF}$ and the $\chi^{2}$ distribution is consistent with the $42 \mathrm{MiniBooNE}$ and SciBooNE antineutrino energy bins minus the two DOF from the oscillation parameters. The test demonstrates how a signal at $\Delta m^{2}=10.0$ $\mathrm{eV}^{2}$ and $\sin ^{2} 2 \theta=0.2$ would be distinguished and found if it existed in the data. 


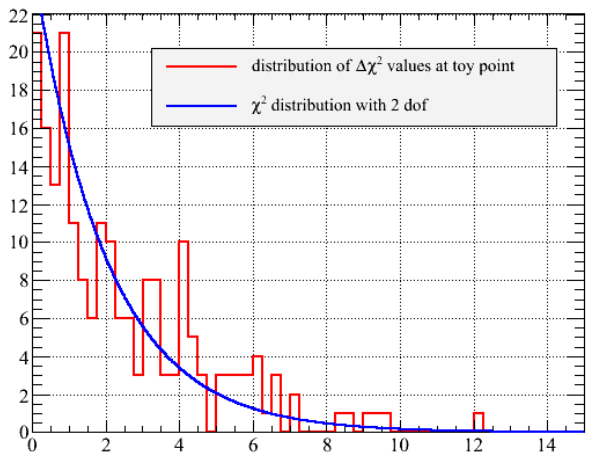

(a) Fake Data $\Delta \chi^{2}$ Values

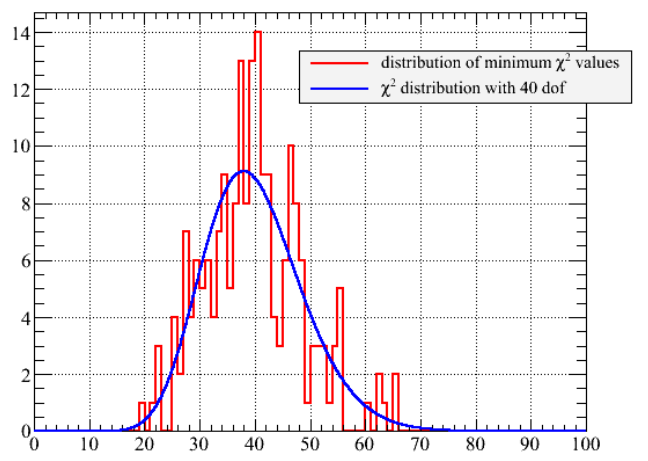

(b) Fake Data $\chi^{2}$ Values

Figure 7.6: Fake data $\Delta \chi^{2}$ distribution with the blue line showing a $\chi^{2}$ distribution with 2 DOF (left) and fake data $\chi^{2}$ distribution with the blue line showing a $\chi^{2}$ distribution with 40 DOF right for many fake data sets and MC prediction all with oscillation signal of $\Delta m^{2}=1.0 \mathrm{eV}^{2}$ and $\sin ^{2} 2 \theta=0.2$ assumed.

Figure 7.11 shows the MC predictions of the deficit expected in the antineutrino energy spectrum due to the different $\sin ^{2} 2 \theta$ and $\Delta m^{2}$ oscillation signals from the fake data studies described earlier in this section normalized to the default MC prediction at the SciBooNE and MiniBooNE detectors. Much can be learned and understood by examining the figure. If the oscillation signal has a $\Delta m^{2}=1.0 \mathrm{eV}^{2}$, SciBooNE acts as a near detector measuring the un-oscillated antineutrino energy spectrum while MiniBooNE acts as a far detector measuring the oscillated, deficient antineutrino energy spectrum. If the oscillation signal has a larger $\Delta m^{2}=10.0 \mathrm{eV}^{2}$, both SciBooNE and MiniBooNE are measuring the oscillated, deficient antineutrino energy spectrum. $\sin ^{2} 2 \theta$ affects the magnitude of the antineutrino energy spectrum deficit and not the shape, as can be seen between the histograms with different $\sin ^{2} 2 \theta$ and the same $\Delta m^{2}$. This is what is expected if the oscillation probability formula in Equation 7.1 is closely examined since the $\sin ^{2} 2 \theta$ acts as a coefficient of the $\sin ^{2} \frac{1.27 \Delta m^{2} L}{E}$ term with the distance and antineutrino energy dependence.

Figure 7.12 shows several fake data exclusion limit curves at the $90 \%$ confidence level (any region enclosed to the right of a curve is excluded with at least a $90 \%$ confidence level for that curve) assuming no oscillation signal for $\Delta m^{2}$ between $0.01 \mathrm{eV}^{2}$ and $100 \mathrm{eV}^{2}$ and 
Average $90 \%$ and $3 \sigma \mathrm{CL}$ for fake data (oscillations)

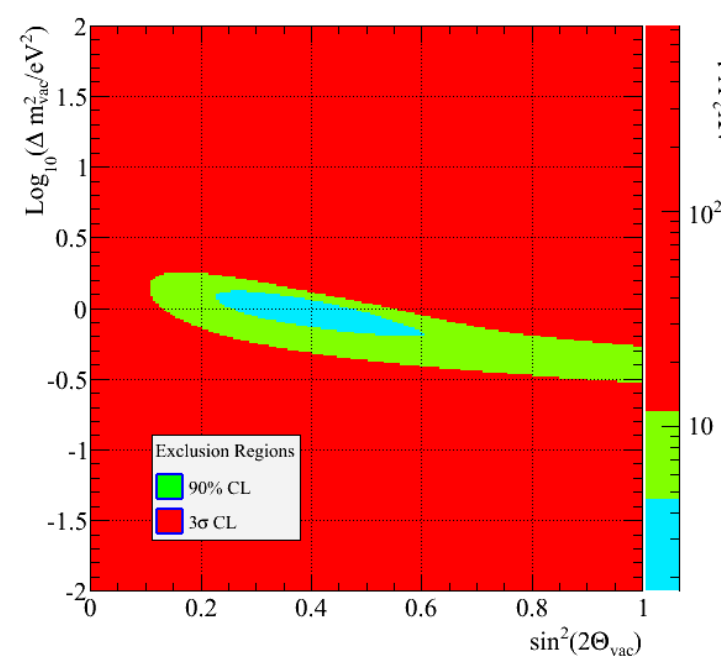

(a) Exclusion Limit From Fake Data Sets Assuming Oscillation Signal With $\Delta m^{2}=1.0 \mathrm{eV}^{2}$ and $\sin ^{2} 2 \theta=$ best fit points

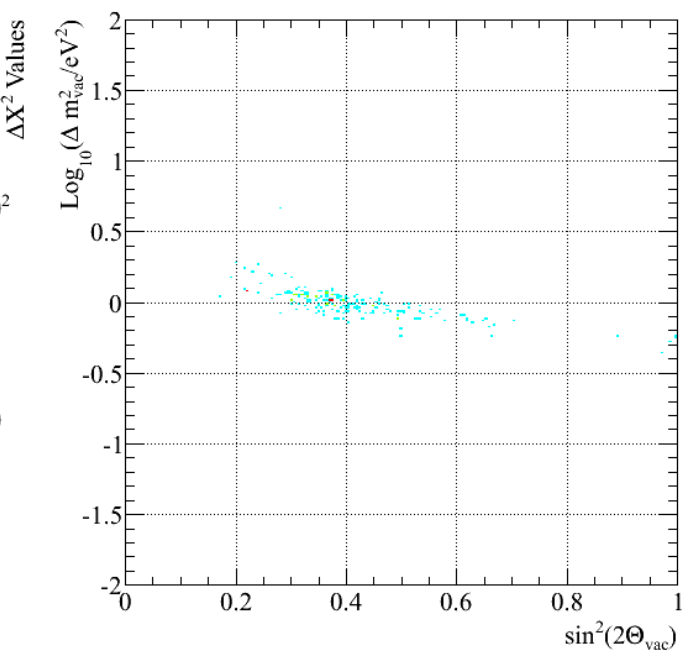

(b) Best Fit $\Delta \chi^{2}$ Points From Fake Data Sets 0.4

Figure 7.7: Averaged oscillation signal region and exclusion limits at the $90 \%$ and 3- $\sigma$ confidence levels (left) and best fit $\Delta \chi^{2}$ points (right) for many fake data sets all with oscillation signal of $\Delta m^{2}=1.0 \mathrm{eV}^{2}$ and $\sin ^{2} 2 \theta=0.4$ assumed. The allowed oscillation signal region is a light blue. The regions were produced assuming the $\Delta \chi^{2}$ distribution is a $\chi^{2}$ distribution with 2 DOF.

$\Delta m^{2}$ between $100 \mathrm{eV}^{2}$ and $10000 \mathrm{eV}^{2}$. If the oscillation parameter space is extended towards larger $\Delta m^{2}$, the oscillation disappearance probability would oscillate much more frequently over the same distance given the energy of the selected antineutrino events. Larger than a certain $\Delta m^{2}$ value, the oscillations would eventually be so short in distance that the reconstruction antineutrino energy distributions in SciBooNE and MiniBooNE would not be able to resolve any oscillation shape at all. All the shape information contained in the antineutrino energy distribution would be irrelevant and only the normalization of the data relative to the $\mathrm{MC}$ prediction can have any resolving power. This effect can be seen in the figure at $\Delta m^{2}$ values larger than $100 \mathrm{eV}^{2}$ where the exclusion limit curves are all constant $\sin ^{2} 2 \theta$ regardless of $\Delta m^{2}$ value, which implies that the antineutrino energy distribution 


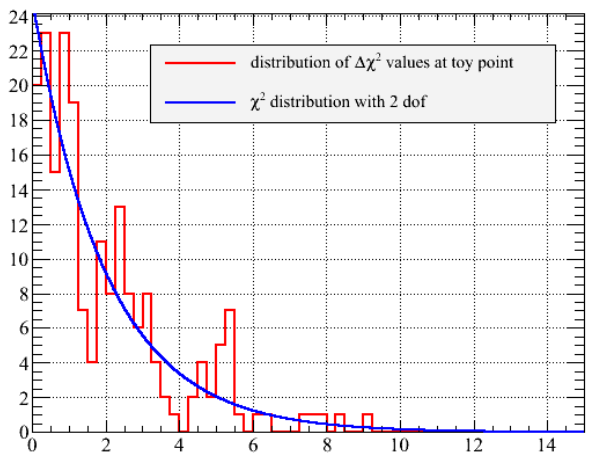

(a) Fake Data $\Delta \chi^{2}$ Values

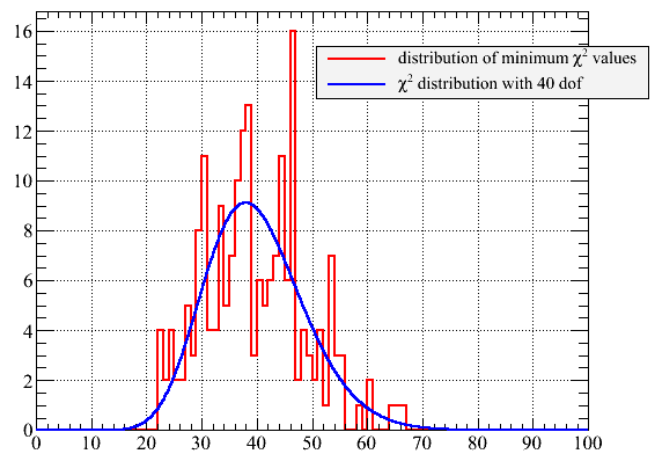

(b) Fake Data $\chi^{2}$ Values

Figure 7.8: Fake data $\Delta \chi^{2}$ distribution with the blue line showing a $\chi^{2}$ distribution with 2 DOF (left) and fake data $\chi^{2}$ distribution with the blue line showing a $\chi^{2}$ distribution with 40 DOF right for many fake data sets and MC prediction all with oscillation signal of $\Delta m^{2}=1.0 \mathrm{eV}^{2}$ and $\sin ^{2} 2 \theta=0.4$ assumed.

shape and hence, the $\Delta m^{2}$ value, no longer has any effect in determining the exclusion limit. This is a good consistency test for the analysis, showing good agreement of what is expected and what is observed.

All the fake data studies show that the $\Delta \chi^{2}$ test statistic is behaving exactly as expected in the oscillation parameter space. The data can now be unveiled and compared to the MC prediction to explore if a $\bar{\nu}_{\mu}$ disappearance oscillation signal exists. 


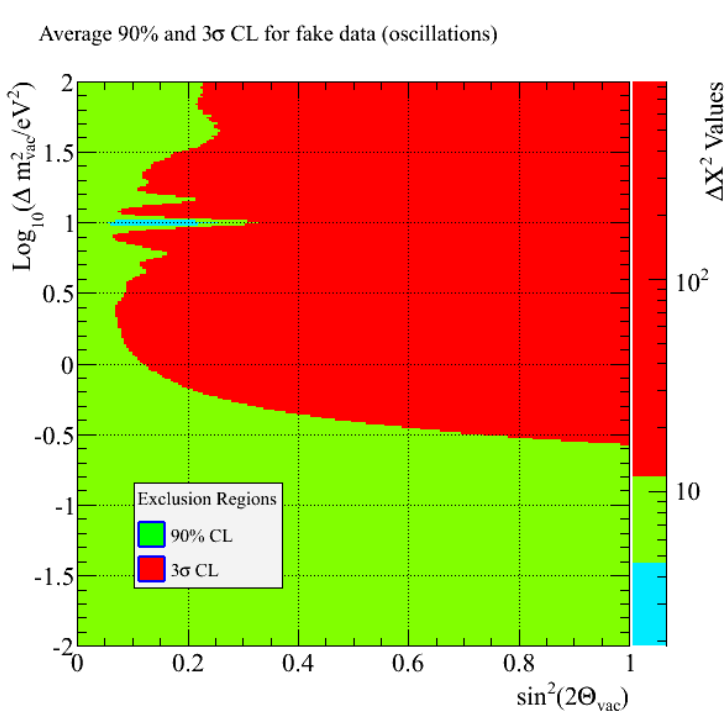

(a) Exclusion Limit From Fake Data Sets Assuming Oscillation Signal With $\Delta m^{2}=10.0 \mathrm{eV}^{2}$ and $\sin ^{2} 2 \theta=0.2$ best fit points

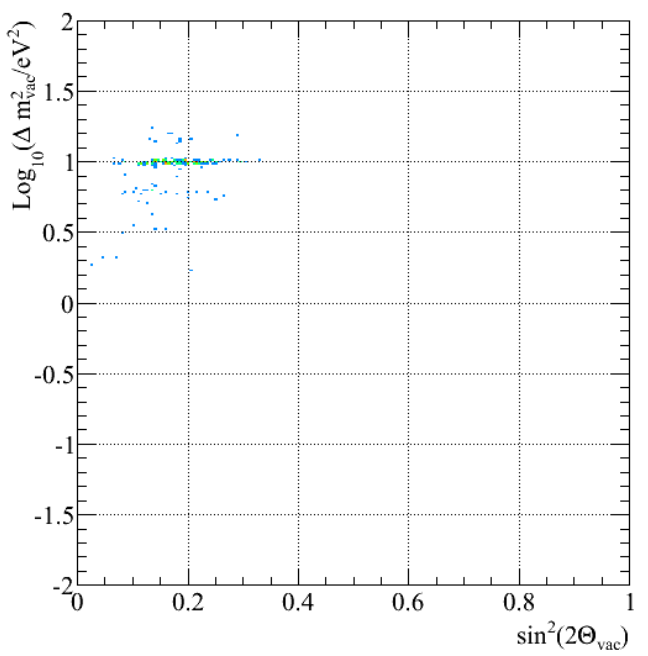

(b) Best Fit $\Delta \chi^{2}$ Points From Fake Data Sets

Figure 7.9: Averaged exclusion limits at the $90 \%$ and $3-\sigma$ confidence levels and possible oscillation signal region (left) and best fit $\Delta \chi^{2}$ points (right) for many fake data sets all with oscillation signal of $\Delta m^{2}=10.0 \mathrm{eV}^{2}$ and $\sin ^{2} 2 \theta=0.2$ assumed. The allowed oscillation signal region is a light blue. The regions were produced assuming the $\Delta \chi^{2}$ distribution is a $\chi^{2}$ distribution with 2 DOF. 


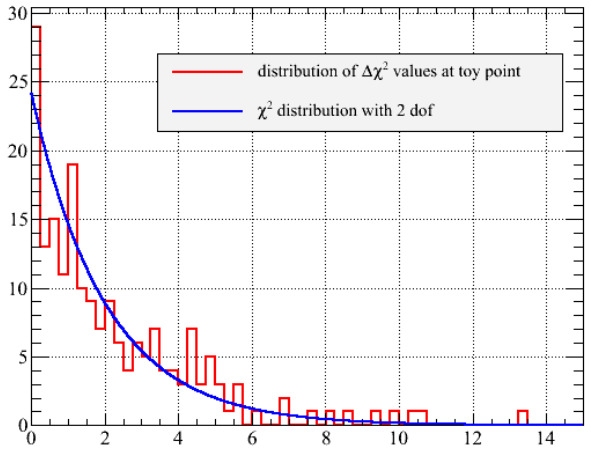

(a) Fake Data $\Delta \chi^{2}$ Values

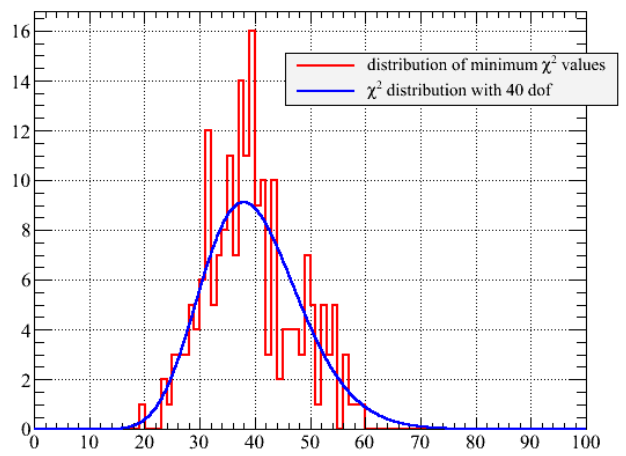

(b) Fake Data $\chi^{2}$ Values

Figure 7.10: Fake data $\Delta \chi^{2}$ distribution with the blue line showing a $\chi^{2}$ distribution with 2 DOF (left) and fake data $\chi^{2}$ distribution with the blue line showing a $\chi^{2}$ distribution with $40 \mathrm{DOF}$ right for many fake data sets and $\mathrm{MC}$ prediction all with oscillation signal of $\Delta m^{2}=10.0 \mathrm{eV}^{2}$ and $\sin ^{2} 2 \theta=0.2$ assumed. 


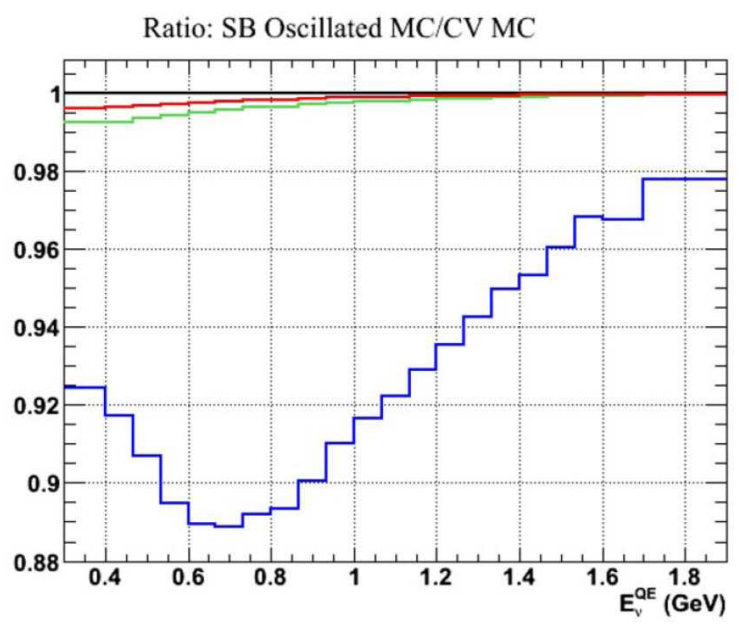

(a) SciBooNE MC Predictions

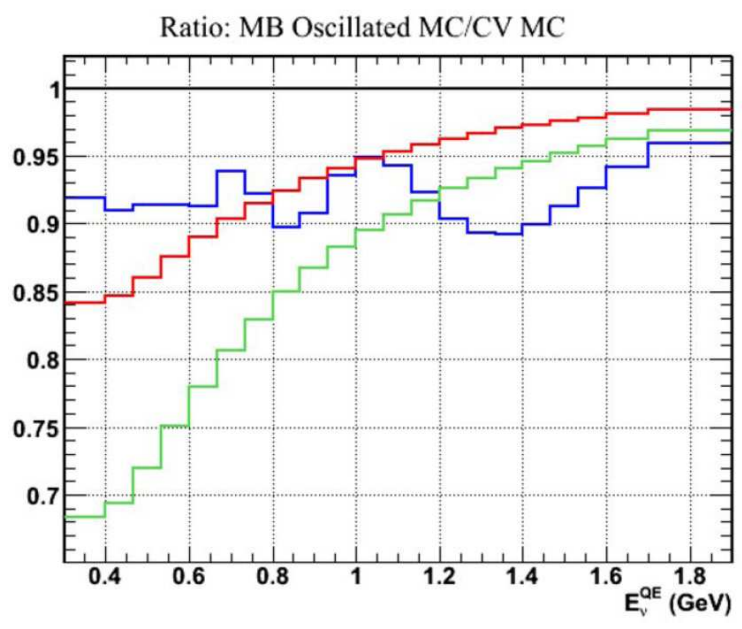

(b) MiniBooNE MC Predictions

Figure 7.11: MC predictions of the deficit expected in the antineutrino energy spectrum due to several different $\sin ^{2} 2 \theta$ and $\Delta m^{2}$ oscillation signals normalized to default MC prediction (no oscillation signal) at the SciBooNE and MiniBooNE detectors. The black line at 1 represents the default MC prediction. The red histogram represents an oscillation signal with $\Delta m^{2}=1.0 \mathrm{eV}^{2}$ and $\sin ^{2} 2 \theta=0.2$. The green histogram represents an oscillation signal with $\Delta m^{2}=1.0 \mathrm{eV}^{2}$ and $\sin ^{2} 2 \theta=0.4$. The blue histogram represents an oscillation signal with $\Delta m^{2}=10.0 \mathrm{eV}^{2}$ and $\sin ^{2} 2 \theta=0.2$. 

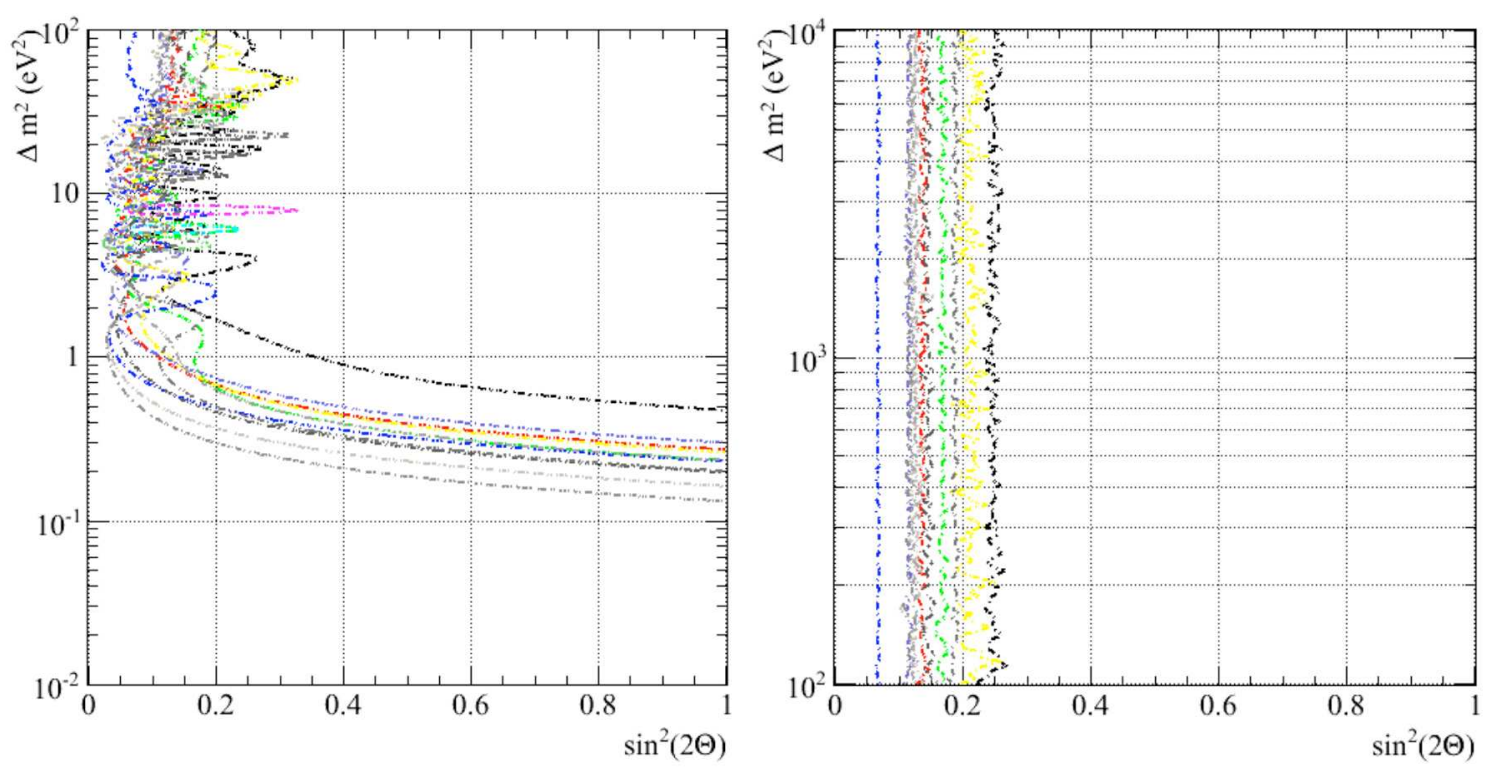

(a) Fake Data Exclusion Limits Assuming No Os- (b) Fake Data Exclusion Limits Assuming No Oscilcillation Signal For $\Delta m^{2}$ Between $0.01 \mathrm{eV}^{2}$ and 100 lation Signal For $\Delta m^{2}$ Between $100 \mathrm{eV}^{2}$ and 10000 $\mathrm{eV}^{2}$

$\mathrm{eV}^{2}$

Figure 7.12: Fake data exclusion limits at the $90 \%$ confidence level assuming no oscillation signal for $\Delta m^{2}$ between $0.01 \mathrm{eV}^{2}$ and $100 \mathrm{eV}^{2}$ (left) and $\Delta m^{2}$ between $100 \mathrm{eV}^{2}$ and 10000 $\mathrm{eV}^{2}$ (right). The regions were produced assuming the $\Delta \chi^{2}$ distribution is a $\chi^{2}$ distribution with 2 DOF. 


\subsection{Results}

The data can now be applied to the analysis to determine the final results. The data is first processed through the event selection criteria described in Chapter 5 to obtain the selected data samples with the high $\bar{\nu}_{\mu}$ event purity. Figure 7.13 shows the observed data event distributions for MiniBooNE and SciBooNE along with the default MC predictions (no disappearance oscillations), in bins of reconstructed antineutrino energy. The systematic uncertainties shown for the MC predictions was computed as just the square roots of the diagonals of the total systematic error matrix without correlations between different antineutrino energy bins. A data over MC prediction excess is observed across the entire antineutrino energy spectrum for both SciBooNE and MiniBooNE and this effect has been observed in previous SciBooNE and MiniBooNE measurements [52; 105. This discrepancy is understood to be due to a lack of understanding in our antineutrino flux and antineutrino interaction (includes cross section and subsequent nuclear interactions) predictions. The use of both SciBooNE and MiniBooNE data, which are two very different distances away from the antineutrino production source, in conjunction in this analysis removes much of antineutrino flux and interaction uncertainties that are the source of the discrepancy. Note that the data over MC excess is still well within the statistical and systematic uncertainties as indicated by the vertical error bars on data (statistical uncertainties) and the shaded error bands on MC (systematic uncertainties) in the figure.

Table 7.1 lists the event counts in each reconstructed antineutrino energy bin used in the $\Delta \chi^{2}$ test statistic, for data, MC predictions, and uncertainties. The listed uncertainties are based on the square roots of the diagonals of the total error matrix and do not include the correlations between different antineutrino energy bins. The reported SciBooNE data has its cosmic data background removed. The cosmic data background is estimated by applying the same selection criteria to a beam-off time window that is five times longer than the beam-on window (for better statistics) and dividing the result by five, resulting in a fractional number of events (described in Subsection 5.4.4).

When the actual data (not the MC generated fake data) is applied into the analysis using the Feldman-Cousins method and the $\Delta \chi^{2}$ and $90 \%$ exclusion confidence levels are calculated throughout the oscillation parameter space, the final result shows no $\bar{\nu}_{\mu} \rightarrow \nu_{x}$ 
Table 7.1: Observed event counts for each MiniBooNE and SciBooNE reconstructed antineutrino energy bin, for data, MC predictions, and uncertainties.

\begin{tabular}{c|c|c|c|c|c|c|c}
\hline \hline $\begin{array}{c}\text { Bin Range } \\
(\mathrm{MeV})\end{array}$ & MB & MB & MB & SB & SB Cosmic & SB & SB \\
\hline $300-400$ & 565 & 413.5 & 111.0 & 1077.0 & 21.0 & 997.3 & 136.8 \\
$400-467$ & 2577 & 2139.2 & 464.8 & 966.8 & 89.2 & 915.6 & 141.9 \\
$467-533$ & 4433 & 4039.9 & 802.2 & 872.8 & 85.2 & 834.4 & 132.1 \\
\hline $533-600$ & 5849 & 5211.0 & 1005.7 & 854.4 & 72.6 & 809.4 & 132.2 \\
$600-667$ & 6411 & 5725.6 & 1108.7 & 856.8 & 59.2 & 790.6 & 137.3 \\
$667-733$ & 6445 & 5778.3 & 1130.3 & 915.0 & 51.0 & 781.9 & 144.3 \\
\hline $733-800$ & 6090 & 5586.8 & 1096.9 & 849.8 & 52.2 & 757.3 & 139.5 \\
$800-867$ & 5678 & 5268.3 & 1044.8 & 876.6 & 43.4 & 717.1 & 138.8 \\
$867-933$ & 5314 & 4826.2 & 951.8 & 787.0 & 39.0 & 655.8 & 138.0 \\
\hline $933-1000$ & 4624 & 4319.6 & 865.1 & 688.0 & 35.0 & 639.7 & 129.6 \\
$1000-1067$ & 4015 & 3720.3 & 747.2 & 628.0 & 29.0 & 580.2 & 125.4 \\
$1067-1133$ & 3349 & 3163.6 & 642.1 & 569.6 & 28.4 & 488.7 & 105.8 \\
\hline $1133-1200$ & 2965 & 2655.9 & 554.3 & 496.6 & 21.4 & 403.9 & 92.2 \\
$1200-1267$ & 2464 & 2147.2 & 453.0 & 377.0 & 23.0 & 308.4 & 74.6 \\
$1267-1333$ & 1937 & 1726.4 & 367.8 & 273.6 & 22.4 & 228.4 & 53.6 \\
\hline $1333-1400$ & 1534 & 1372.0 & 297.9 & 178.6 & 18.4 & 150.0 & 37.2 \\
$1400-1467$ & 1227 & 1073.3 & 238.1 & 111.2 & 18.8 & 89.4 & 23.9 \\
$1467-1533$ & 859 & 820.5 & 187.7 & 65.4 & 17.6 & 57.1 & 15.0 \\
\hline $1533-1600$ & 679 & 607.2 & 145.8 & 39.0 & 17.0 & 33.1 & 10.4 \\
$1600-1700$ & 684 & 607.2 & 149.1 & 40.8 & 28.2 & 27.6 & 9.9 \\
\hline \hline $1700-1900$ & 610 & 560.1 & 144.5 & 37.6 & 39.4 & 24.8 & 7.8 \\
\hline
\end{tabular}

2 The SB data has its SB cosmic data background removed.

oscillation within the sensitivity of the analysis. Figure 7.14 and Figure 7.15 show the $\bar{\nu}_{\mu}$ 90\% CL exclusion disappearance limit for the joint disappearance analysis in linear scale and 
$\log$ scale, respectively. Regions with very small $\sin ^{2} 2 \theta$ and small $\Delta m^{2}$ cannot be excluded due to the lack of sensitivity of the analysis as shown by the fake data frequentist studies described in Section 7.4. The 90\% CL exclusion limit leans towards the more sensitive edge of the expected $90 \%$ CL sensitivity determined from fake data studies and shown in the shaded band in both figures. This makes sense since what is observed is a data excess above default MC prediction while what is expected from a $\bar{\nu}_{\mu}$ disappearance is a deficit of data relative to default MC prediction. This pushes the $90 \%$ CL exclusion limit towards the more sensitive side. For $\Delta m^{2}=1 \mathrm{eV}^{2}$ and $\Delta m^{2}=10 \mathrm{eV}^{2}$, the $90 \%$ CL limit for $\sin ^{2} 2 \theta$ are at 0.121 and 0.024 , respectively. At $\sin ^{2} 2 \theta=1$, the $90 \%$ CL limit for $\Delta m^{2}$ is $0.156 \mathrm{eV}^{2}$. The best fit point from the joint analysis is $\Delta m^{2}=5.9 \mathrm{eV}^{2}, \sin ^{2} 2 \theta=0.086$. At the best fit point, $\chi^{2}=40.0$ (probability $47.1 \%$ ). For the null oscillation hypothesis, the $\chi^{2}$ is slightly higher as expected at $\chi^{2}=43.5$ (probability $41.2 \%$ ). With $\Delta \chi^{2}=3.5$, the null oscillation hypothesis is excluded at $81.9 \% \mathrm{CL}$, though this is just the result of the fluctuations inherent in the data. Figure 7.14 and Figure 7.15 also show the improvement in the $90 \%$ confidence level exclusion limit for $\bar{\nu}_{\mu}$ disappearance relative to previous MiniBooNE 38 and CCFR experiments 40 .

A MiniBooNE-only disappearance analysis is included to give a sense of what the sensitivity would be without the inclusion of SciBooNE data. Figure 7.16 shows the $90 \%$ CL exclusion region and best fit point for the MiniBooNE-only $\bar{\nu}_{\mu}$ disappearance analysis, completed using the same methodology as the joint disappearance analysis except with the exclusion of SciBooNE data, SciBooNE MC prediction, and SciBooNE portion of the error matrix uncertainties in the $\chi^{2}$ statistic. The best fit point is $\Delta m^{2}=5.9 \mathrm{eV}^{2}, \sin ^{2} 2 \theta=0.076$. At the best fit point, $\chi^{2}=25.7$ (probability 12.4\%). For the null oscillation hypothesis, $\chi^{2}=28.3$ (probability $13.7 \%$ ). With $\Delta \chi^{2}=2.6$, the null oscillation hypothesis is excluded at $52.4 \%$ CL. A significant portion of the overall joint analysis's exclusion limit can be derived from the MiniBooNE-only analysis's exclusion limit, which makes sense since the selected data set from MiniBooNE is by far the largest giving it the greatest predictive power. Again, Figure 7.16 also shows the improvement in the $90 \%$ confidence level exclusion limit for $\bar{\nu}_{\mu}$ disappearance relative to previous MiniBooNE 38] and CCFR experiments 40. The weakness of the MiniBooNE-only disappearance analysis is that it is more dependent 
on the understanding of the antineutrino flux and antineutrino interaction uncertainties. The joint disappearance analysis removes this weakness since the SciBooNE data is used to constrain the antineutrino flux and antineutrino interaction uncertainties and is therefore more rigorous.

Figure 7.17 shows the data to $\mathrm{MC}$ ratios for MiniBooNE and SciBooNE, as well as how the best fit signal modifies the MC predictions. From these ratio plots, it can be seen that MiniBooNE and SciBooNE have correlated data to MC ratios. Both ratio plots show data to $\mathrm{MC}$ ratios of over 1 , or a data over MC excess. Figure 7.18 shows the double ratio defined as:

$$
\frac{\left(\frac{\text { MiniBooNE data }}{\text { MiniBooNE default MC }}\right)}{\left(\frac{\text { SciBooNE data }}{\text { SciBooNE default MC }}\right)} \text {. }
$$

In the double ratio, any common normalization differences between MiniBooNE and SciBooNE data over MC are removed. Many of the normalization differences between MiniBooNE and SciBooNE are caused by systematic uncertainties from the same sources and therefore, cancel out in the double ratio and the expected result is a value near one. The double ratio result agrees well with the expectation except where statistics are small. Also, the best fit result is in line with data and an expectation of one. Therefore, the double ratio result shows that using both SciBooNE and MiniBooNE takes advantage of correlations between SciBooNE and MiniBooNE, which can be used to perform a better disappearance analysis that a MiniBooNE-only disappearance analysis.

The results of the data show that no $\bar{\nu}_{\mu} \rightarrow \nu_{x}$ oscillation is discovered within the sensitivity of the analysis. Post data unveiling consistency checks show that the data behaves in the manner expected. There is a common upward normalization shift of data relative to MC prediction for both MiniBooNE and SciBooNE, which has been previously observed 52 , 105. The discrepancy is attributed to our lack of understanding of antineutrino flux and antineutrino interaction with one possible major contribution being the limitations of the RFG model to simulate what is happening in the carbon targets, including multi-nucleon nuclear effects (described in Section). The use of both SciBooNE and MiniBooNE in conjunction resolves this issue since these uncertainties cancel out in the analysis. 


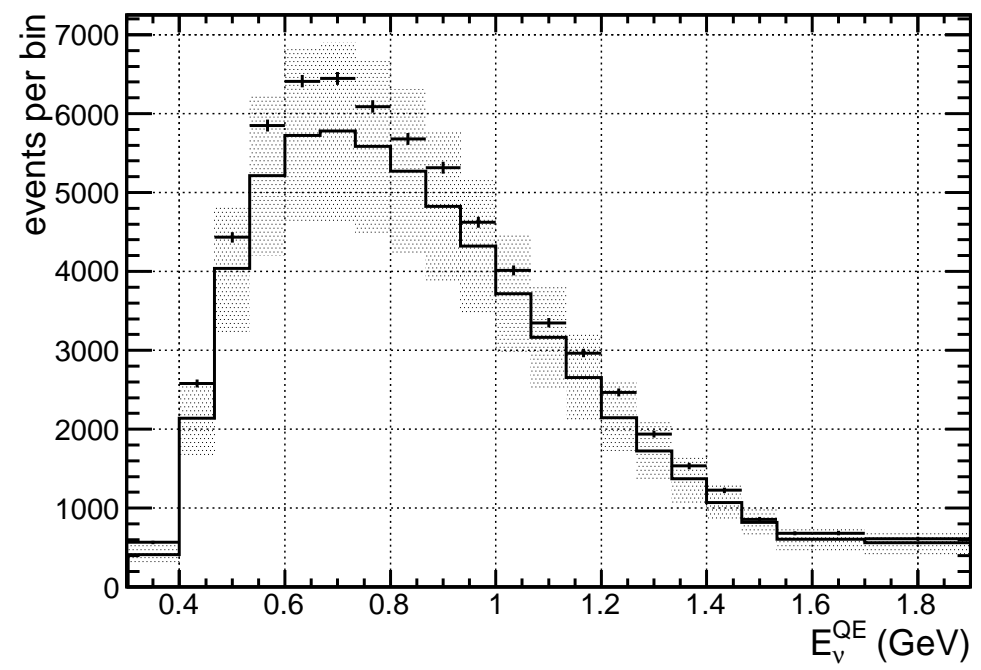

(a) MiniBooNE

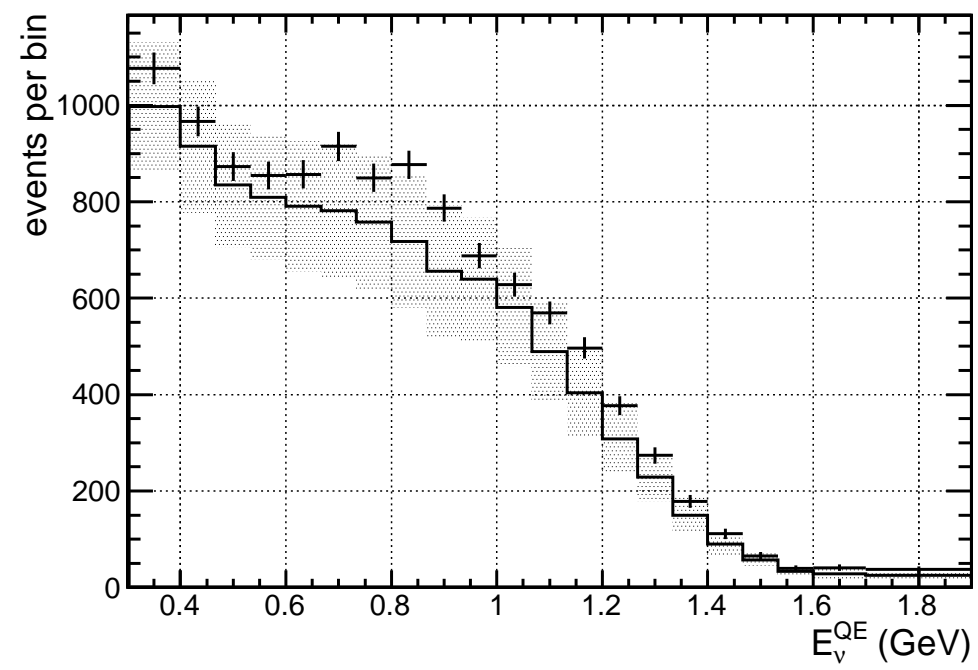

(b) SciBooNE

Figure 7.13: Reconstructed antineutrino energy $\left(E_{\nu}^{Q E}\right)$ distribution for data events (crosses), compared to MC predictions (solid lines), for MiniBooNE and SciBooNE. Vertical error bars on data are the statistical uncertainties. Shaded error bands around the MC predictions are the systematic uncertainties computed as the square roots of the diagonals of the total systematic error matrix. 


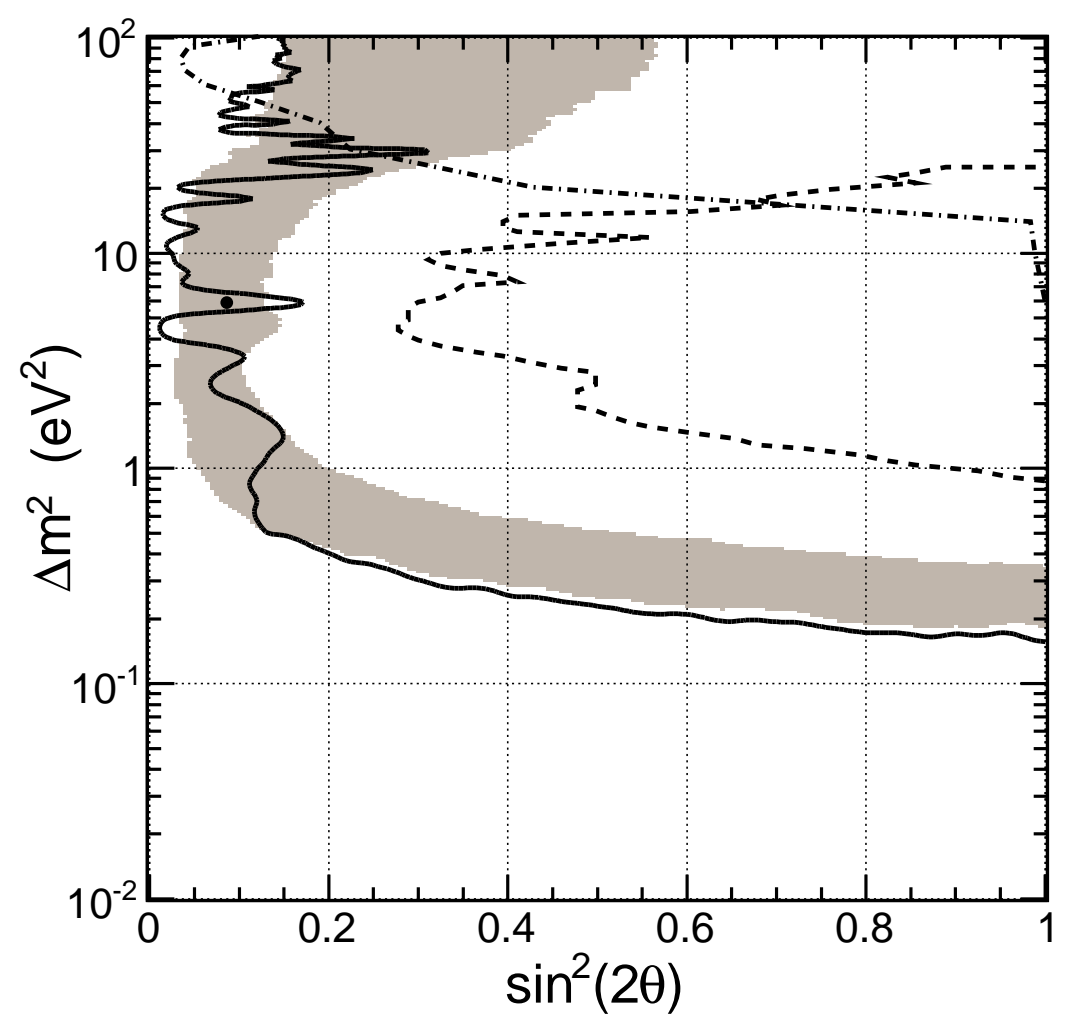

Figure 7.14: 90\% CL exclusion region (solid line) and best fit point for the joint MiniBooNE, SciBooNE $\bar{\nu}_{\mu}$ disappearance analysis in linear scale for $\sin ^{2} 2 \theta$ and $\Delta m^{2}$. Also shown is the 90\% CL result from the 2009 MiniBooNE disappearance analysis [38] (dashed line) and the CCFR experiment [40] (dot-dashed line). The expected 90\% CL sensitivity band from fake data studies is also shown (shaded region); $1 \sigma(68 \%)$ of fake data tests, where the fake data had statistical and systematic fluctuations but no oscillation signal, had 90\% CL limit curves in this shaded region. 


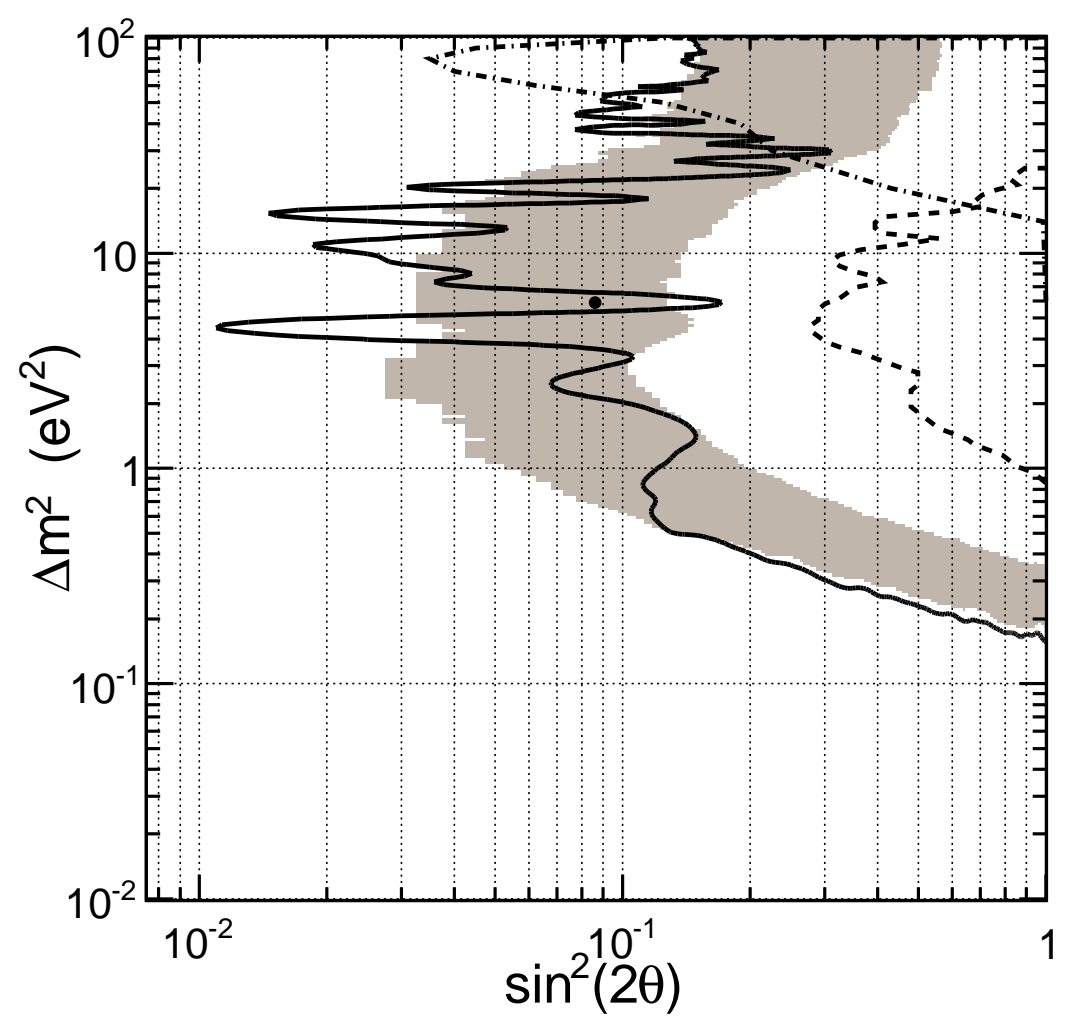

Figure 7.15: 90\% CL exclusion region (solid line) and best fit point for the joint MiniBooNE, SciBooNE $\bar{\nu}_{\mu}$ disappearance analysis in log scale for $\sin ^{2} 2 \theta$ and $\Delta m^{2}$. Also shown is the 90\% CL result from the 2009 MiniBooNE disappearance analysis [38] (dashed line) and the CCFR experiment [40] (dot-dashed line). The expected 90\% CL sensitivity band from fake data studies is also shown (shaded region); $1 \sigma(68 \%)$ of fake data tests, where the fake data had statistical and systematic fluctuations but no oscillation signal, had 90\% CL limit curves in this shaded region. 


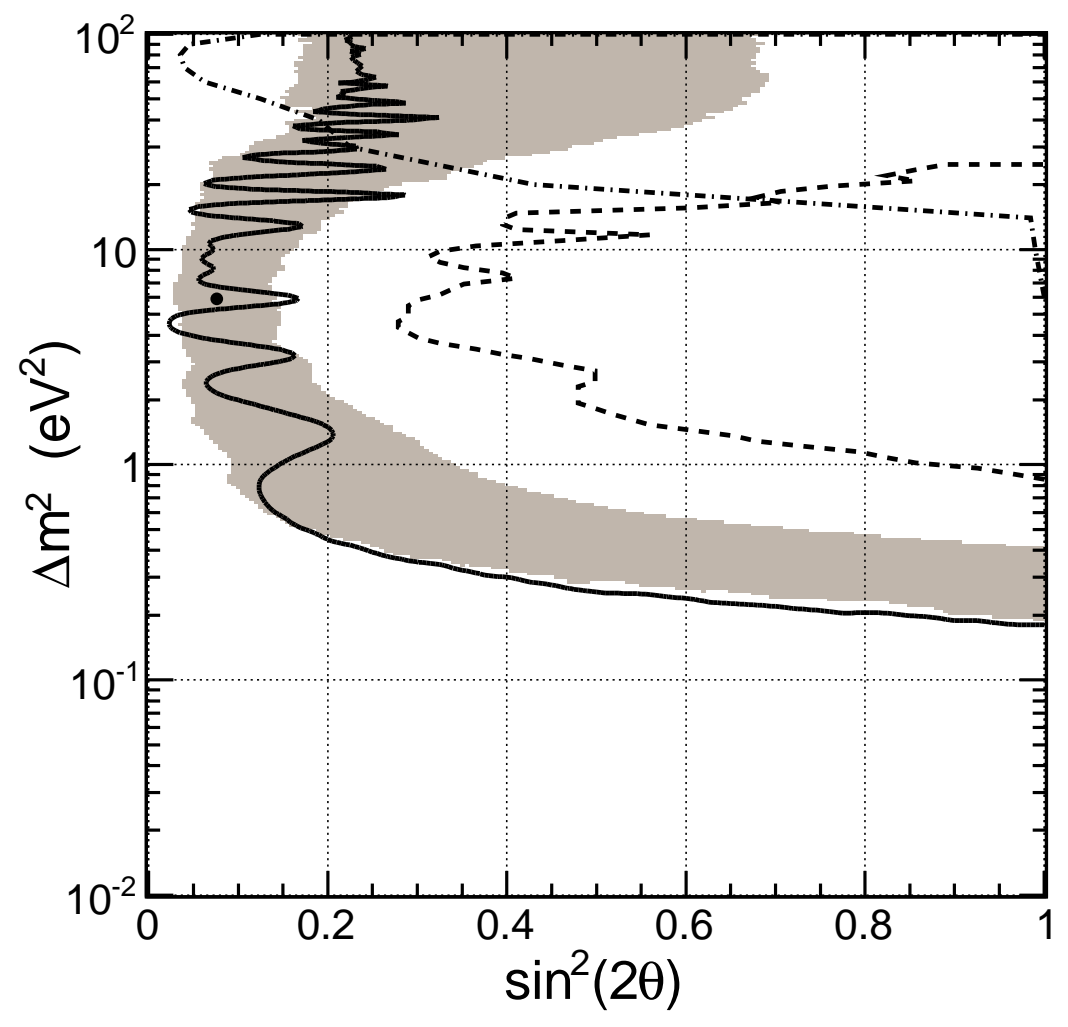

Figure 7.16: 90\% CL exclusion region (solid line) and best fit point for the MiniBooNEonly $\bar{\nu}_{\mu}$ disappearance analysis. Also shown is the $90 \%$ CL result from the 2009 MiniBooNE disappearance analysis [38] (dashed line) and the CCFR experiment [40] (dot-dashed line). The expected $90 \%$ CL sensitivity band from fake data studies is also shown (shaded region); $1 \sigma(68 \%)$ of fake data tests, where the fake data had statistical and systematic fluctuations but no oscillation signal, had 90\% CL limit curves in this shaded region. 


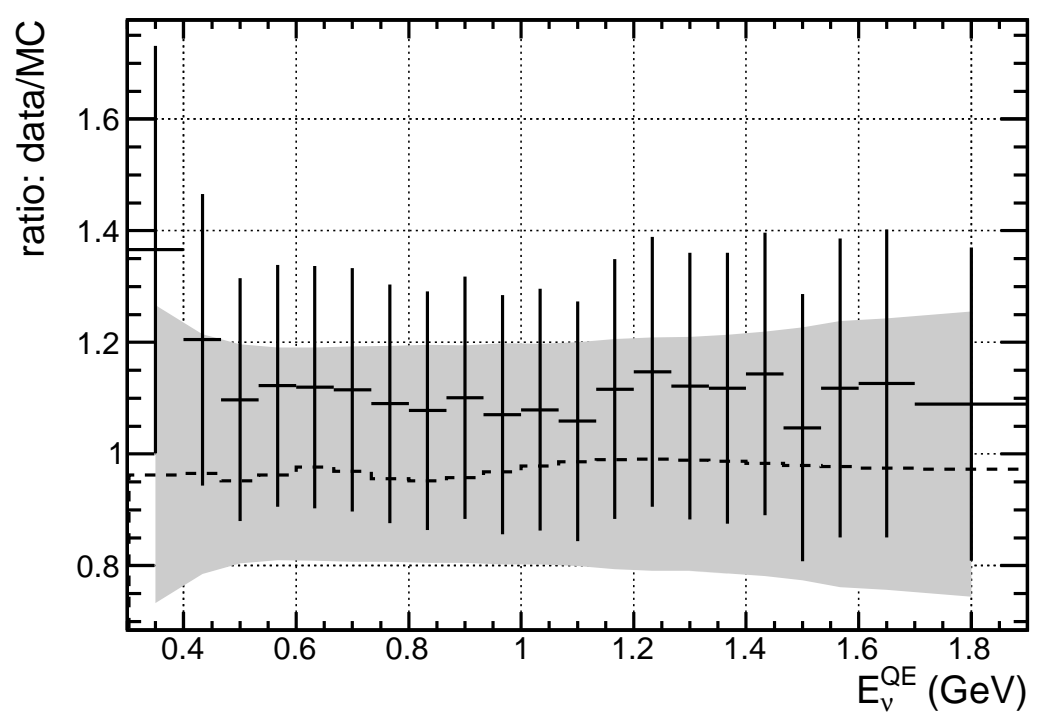

(a) MiniBooNE

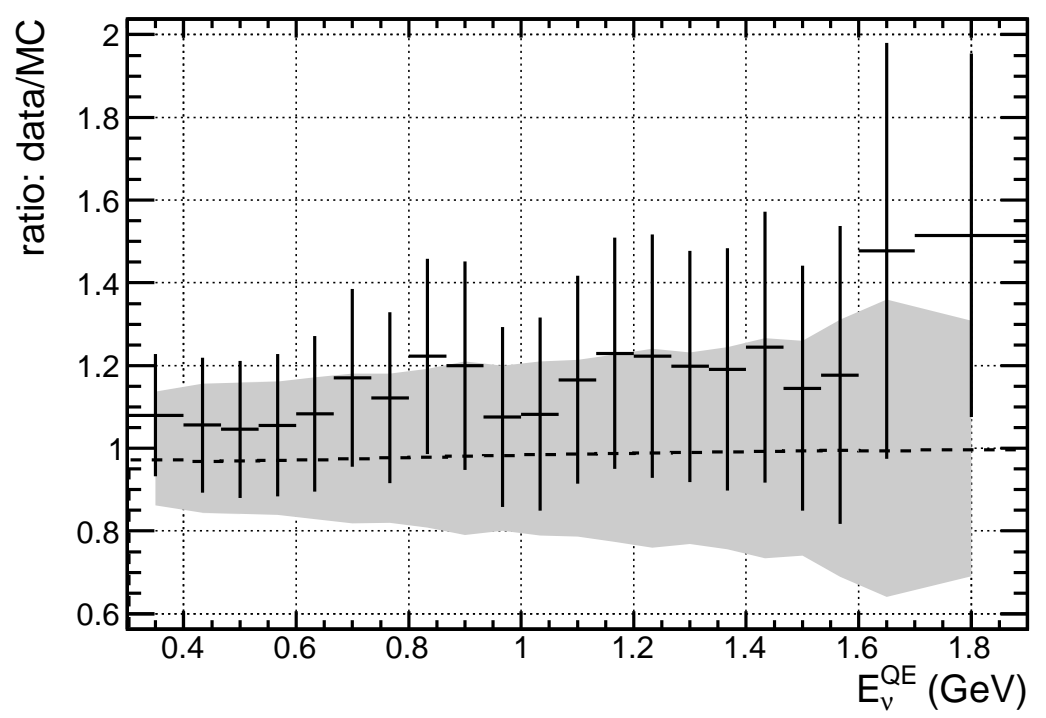

(b) SciBooNE

Figure 7.17: The ratio, with full error bars (systematic and statistical), between data and default $\mathrm{MC}$ as a function of reconstructed antineutrino energy $\left(E_{\nu}^{Q E}\right)$. The ratio of best fit signal MC to default MC is also shown (dashed line). The best fit results from the joint analysis were used to generate the signal MC. The shaded regions are the $1 \sigma$ band from fake data with statistical and systematic fluctuations but no oscillation signal. 


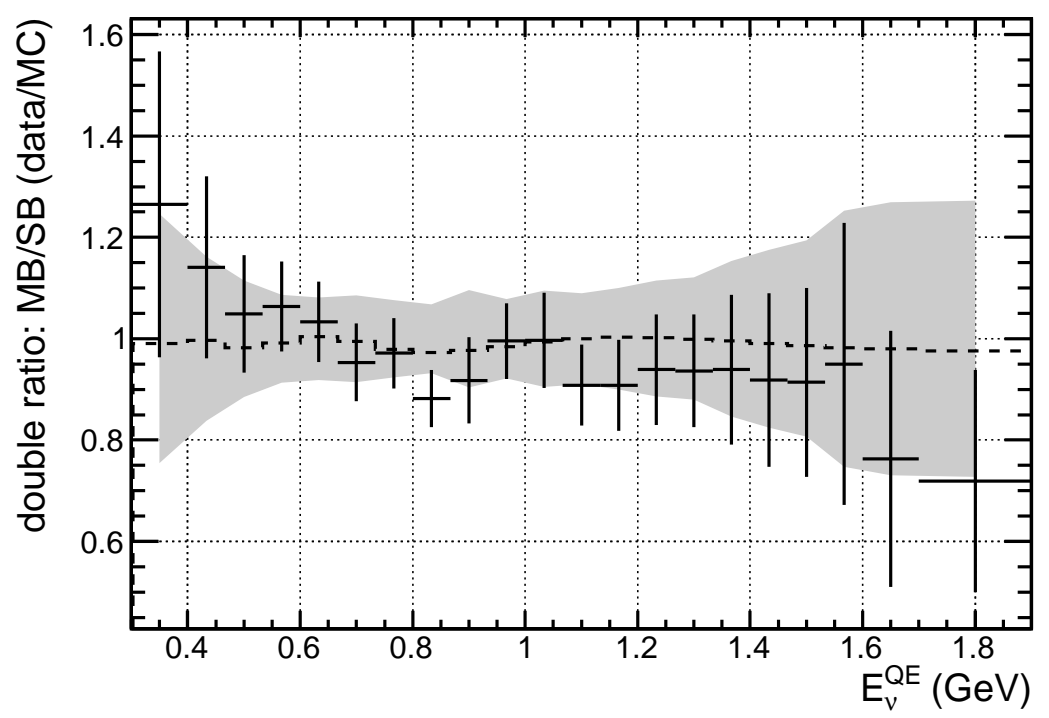

Figure 7.18: The double ratio (Eq. 7.7), with full error bars (systematic and statistical), as a function of reconstructed antineutrino energy $\left(E_{\nu}^{Q E}\right)$. Some of the MiniBooNE and SciBooNE uncertainties cancel in this double ratio. The double ratio where the MiniBooNE and SciBooNE signal MC based on the best fit results from the joint analysis are used in placed of data is also shown (dashed line). The shaded region is the $1 \sigma$ band from fake data with statistical and systematic fluctuations but no oscillation signal. 


\section{Chapter 8}

\section{Conclusions}

A search for $\bar{\nu}_{\mu}$ disappearance into a sterile neutrino has been performed in the $\Delta m^{2}$ region between 0.01 and $100 \mathrm{eV}^{2}$ using both the SciBooNE and MiniBooNE detectors in conjunction. The use of both SciBooNE and MiniBooNE data allows the cancellation of much of the antineutrino flux and interaction uncertainties. The reason is because the SciBooNE detector acts as a near detector that measures the antineutrino flux and constrains its uncertainties while the MiniBooNE acts as a far detector that measures the disappearance oscillation. This results in a more rigorous analysis and better sensitivity than just MiniBooNE data alone. The joint analysis shows no evidence of sterile neutrino oscillations and the data and MC prediction assuming no sterile neutrino oscillations agree with each other within uncertainties for both SciBooNE and MiniBooNE detectors. New sterile neutrino oscillation parameter space has been excluded at the 90\% CL as shown in Figure 7.14. In fact, assuming CPT conservation, the $90 \%$ CL limit for the $\bar{\nu}_{\mu}$ disappearance search covers more sterile neutrino parameter space than all previous neutrino or antineutrino disappearance searches.

The $\bar{\nu}_{\mu}$ disappearance analysis can be combined with other world measurement on neutrino and antineutrino disappearance (assuming CPT conservation) to produce an even greater sensitivity for sterile neutrino search. In addition, there are recent studies being conducted to better understand neutrino and antineutrino interactions in the nucleus and to reduce neutrino and antineutrino cross sectional uncertainties. If realized, these future improvements in our understanding of physics associated with neutrino and antineutrino in- 
teractions will lead to ever more sensitive neutrino and antineutrino disappearance searches and open up new measurable regions in the sterile neutrino oscillation parameter space that are unfeasible today.

The importance of more sensitive neutrino and antineutrino disappearance searches can not be understated. Disappearance searches, along with their complementary appearance searches, are essential to discovering the presence of neutrino oscillations into sterile neutrinos. Disappearance and appearances searches together constrain the mixing parameters of sterile neutrinos in the $3+N$ sterile neutrino models, where there are 3 Standard Model neutrino mass eigenstates and $N$ sterile neutrino mass eigenstates. LSND and MiniBooNE measured $\bar{\nu}_{e}\left(\nu_{e}\right)$ appearance in a $\bar{\nu}_{\mu}\left(\nu_{\mu}\right)$ beam consistent with a sterile neutrino oscillation of $\Delta m^{2} \approx 1 \mathrm{eV}^{2}, 23 ; 26$ and recent results showed evidence of $\bar{\nu}_{e}$ disappearance from reactors 114, so it is now more important than ever to find $\nu_{\mu}$ and $\bar{\nu}_{\mu}$ disappearance so that the sterile neutrino mixing parameters can be constrained and sterile neutrino models can be either verified or disproved.

The conclusive discovery of sterile neutrinos through disappearance and appearance searches would be a major step forward in particle physics since it would be particle physics beyond the Standard Model. The search for sterile neutrinos forms a large part of the current particle physics program and this joint MiniBooNE and SciBooNE $\bar{\nu}_{\mu}$ disappearance analysis is an important contribution to the cause. 


\section{Bibliography}

[1] Francis. El experimento RENO confirma el anuncio de Daya Bay tambin con antineutrinos. http://francisthemulenews.wordpress.com/2012/04/06/el-experimento-renoconfirma-el-anuncio-de-daya-bay-para-los-neutrinos-pero-con-antineutrinos/, 2012.

[2] M. Maltoni. Sterile neutrinos after the first MiniBooNE results. J. Phys. Conf. Ser., 110:082011, 2008.

[3] A.A. Aguilar-Arevalo et al. The Neutrino Flux prediction at MiniBooNE. Phys. Rev. D, 79:072002, 2009.

[4] S. Schael et al. Precision electroweak measurements on the $Z$ resonance. Phys. Rept., 427:257-454, 2006.

[5] Ch. Kraus et al. Final results from phase II of the Mainz neutrino mass searchin tritium $\beta$ decay. Eur. Phys. J., C40:447-468, 2005.

[6] K. Assamagan et al. Upper limit of the muon-neutrino mass and charged-pion mass from momentum analysis of a surface muon beam. Phys. Rev. D, 53:6065-6077, Jun 1996.

[7] R. Barate et al. An upper limit on the $\tau$ neutrino mass from three- and five-prong tau decays. Eur. Phys. J., C2:395-406, 1998.

[8] B.T. Cleveland et al. Measurement of the solar electron neutrino flux with the Homestake chlorine detector. Astrophys. J., 496:505-526, 1998.

[9] W. Hampel et al. GALLEX solar neutrino observations: Results for GALLEX IV. Phys. Lett. B, 447:127-133, 1999. 
[10] M. Altmann et al. Complete results for five years of GNO solar neutrino observations. Phys. Lett. B, 616:174-190, 2005.

[11] J.N. Abdurashitov et al. Measurement of the solar neutrino capture rate with gallium metal. III: Results for the 2002-2007 data-taking period. Phys. Rev. C, 80:015807, 2009.

[12] B. Aharmim et al. Electron energy spectra, fluxes, and day-night asymmetries of B-8 solar neutrinos from measurements with $\mathrm{NaCl}$ dissolved in the heavy-water detector at the Sudbury Neutrino Observatory. Phys. Rev. C, 72:055502, 2005.

[13] J. Hosaka et al. Solar neutrino measurements in super-Kamiokande-I. Phys. Rev. D, $73: 112001,2006$.

[14] C. Arpesella et al. Direct Measurement of the Be-7 Solar Neutrino Flux with 192 Days of Borexino Data. Phys. Rev. Lett., 101:091302, 2008.

[15] S. Abe et al. Precision Measurement of Neutrino Oscillation Parameters with KamLAND. Phys. Rev. Lett., 100:221803, 2008.

[16] Beringer, J. and others. Review of Particle Physics. Phys. Rev. D, 86:010001, Jul 2012.

[17] Y. Fukuda et al. Atmospheric muon-neutrino / electron-neutrino ratio in the multiGeV energy range. Phys. Lett. B, 335:237-245, 1994.

[18] Ahn, M. H. and others. Measurement of neutrino oscillation by the K2K experiment. Phys. Rev. D, 74:072003, Oct 2006.

[19] Michael, D. G. and others. Observation of Muon Neutrino Disappearance with the MINOS Detectors in the NuMI Neutrino Beam. Phys. Rev. Lett., 97:191801, Nov 2006.

[20] Y. Abe et al. Reactor electron antineutrino disappearance in the Double Chooz experiment. Phys. Rev. D, 86:052008, 2012. 
[21] F.P. An et al. Observation of electron-antineutrino disappearance at Daya Bay. Phys. Rev. Lett., 108:171803, 2012.

[22] J.K. Ahn et al. Observation of Reactor Electron Antineutrino Disappearance in the RENO Experiment. Phys. Rev. Lett., 108:191802, 2012.

[23] A. Aguilar-Arevalo et al. Evidence for neutrino oscillations from the observation of anti-neutrino(electron) appearance in a anti-neutrino(muon) beam. Phys. Rev. D, 64:112007, 2001.

[24] A. A. Aguilar-Arevalo et al. A Search for electron neutrino appearance at the $\Delta m^{2} \sim$ 1eV² scale. Phys. Rev. Lett., 98:231801, 2007.

[25] A. A. Aguilar-Arevalo et al. Event excess in the miniboone search for $\bar{\nu}_{\mu} \rightarrow \bar{\nu}_{e}$ oscillations. Phys. Rev. Lett., 105:181801, Oct 2010.

[26] A.A. Aguilar-Arevalo et al. A Combined $\nu_{\mu}$ to $\nu_{e}$ and $\bar{\nu}_{\mu}$ to $\bar{\nu}_{e}$ Oscillation Analysis of the MiniBooNE Excesses. arXiv:hep-ex/1207.4809, 2012.

[27] Ashie, Y. and others. Measurement of atmospheric neutrino oscillation parameters by Super-Kamiokande I. Phys. Rev. D, 71:112005, Jun 2005.

[28] Y. Declais et al. Search for neutrino oscillations at 15-meters, 40-meters, and 95meters from a nuclear power reactor at Bugey. Nucl. Phys. B, 434:503-534, 1995.

[29] M. Apollonio et al. Search for neutrino oscillations on a long baseline at the CHOOZ nuclear power station. Eur. Phys. J., C27:331-374, 2003.

[30] F. Dydak et al. A Search for Muon-neutrino Oscillations in the $\Delta m^{2}$ Range $0.3-\mathrm{eV}^{2}$ to $90-\mathrm{eV}^{2}$. Phys. Lett. B, 134:281, 1984.

[31] F. Boehm et al. Final results from the Palo Verde neutrino oscillation experiment. Phys. Rev. D, 64:112001, 2001.

[32] B. Armbruster et al. Upper limits for neutrino oscillations $\bar{\nu}_{\mu} \rightarrow \bar{\nu}_{e}$ from muon decay at rest. Phys. Rev. D, 65:112001, 2002. 
[33] P. Astier et al. Search for $\nu_{\mu} \rightarrow \nu_{e}$ oscillations in the NOMAD experiment. Phys.Lett., B570:19-31, 2003.

[34] H. Pas et al. Sterile-active neutrino oscillations and shortcuts in the extra dimension. Phys. Rev. D, 72:095017, 2005.

[35] S. Palomares-Ruiz et al. Explaining LSND by a decaying sterile neutrino. JHEP, 0509:048, 2005.

[36] A. Nelson and J. Walsh. Short Baseline Neutrino Oscillations and a New Light Gauge Boson. Phys. Rev. D, 77:033001, 2008.

[37] K.B.M. Mahn et al. Dual baseline search for muon neutrino disappearance at 0.5eV $\mathrm{eV}^{2}<\Delta m^{2}<40 \mathrm{eV}^{2}$. Phys.Rev., D85:032007, 2012.

[38] A.A. Aguilar-Arevalo et al. Search for Muon Neutrino and Antineutrino Disappearance in MiniBooNE. Phys. Rev. Lett., 103:061802, Aug 2009.

[39] P. Adamson et al. Active to sterile neutrino mixing limits from neutral-current interactions in MINOS. Phys. Rev. Lett., 107:011802, 2011.

[40] I. E. Stockdale et al. Limits on Muon-Neutrino Oscillations in the Mass Range 30 $<\Delta m^{2}<1000 \mathrm{eV}^{2} / \mathrm{c}^{4}$. Phys. Rev. Lett., 52:1384-1388, Apr 1984.

[41] K. Hiraide et al. Search for Charged Current Coherent Pion Production on Carbon in a Few-GeV Neutrino Beam. Phys. Rev. D, 78:112004, 2008.

[42] A.A. Aguilar-Arevalo et al. The MiniBooNE Detector. Nucl.Instrum.Meth., A599:2846, 2009.

[43] K. Nitta et al. The K2K SciBar detector. Nucl. Instrum. Meth., A535:147-151, 2004.

[44] M. Hasegawa. PhD thesis, Kyoto University, 2006.

[45] S. Buontempo et al. Construction and test of calorimeter modules for the CHORUS experiment. Nucl. Instrum. Meth., A349:70-80, 1994. 
[46] M. G. Catanesi et al. Measurement of the production cross-section of positive pions in the collision of $8.9 \mathrm{GeV} / \mathrm{c}$ protons on beryllium. Eur. Phys. J., C52:29-53, 2007.

[47] I. Chemakin et al. Pion production by protons on a thin beryllium target at $6.4,12.3$, and $17.5 \mathrm{GeV} / c$ incident proton momenta. Phys. Rev. C, 77:015209, 2008.

[48] C. Mariani et al. Improved parametrization of $K^{+}$production in $p$-Be collisions at low energy using feynman scaling. Phys. Rev. D, 84:114021, Dec 2011.

[49] F. Abe et al. Production of neutral strange particles $K_{S}^{0}$ and $\Lambda^{0}$ by $12-\mathrm{GeV}$ protons on nuclear targets. Phys. Rev. D, 36:1302-1319, Sep 1987.

[50] N. V. Mokhov et al. MARS Code Developments. arXiv:nucl-th/9812038, 1998.

[51] K. Hiraide. PhD thesis, Kyoto University, 2009.

[52] A.A. Aguilar-Arevalo et al. First measurement of the muon neutrino charged current quasielastic double differential cross section. Phys. Rev. D, 81:092005, May 2010.

[53] D. Allasia et al. Investigation of exclusive channels in neutrino/antineutrino-deuteron charged current interactions. Nuclear Physics B, 343(2):285-309, 1990.

[54] N. J. Baker et al. Quasielastic neutrino scattering: A measurement of the weak nucleon axial-vector form factor. Phys. Rev. D, 23:2499-2505, Jun 1981.

[55] N. J. Baker et al. Total cross sections for $\nu_{\mu} n$ and $\nu_{\mu} p$ charged-current interactions in the 7-foot bubble chamber. Phys. Rev. D, 25:617-623, Feb 1982.

[56] C. Baltay et al. Cross Sections and Scaling-Variable Distributions of Neutral- and Charged- Current Neutrino-Nucleon Interactions from a Low-Energy Narrow Band Beam. Phys. Rev. Lett., 44:916-919, Apr 1980.

[57] S. J. Barish et al. Study of neutrino interactions in hydrogen and deuterium: Description of the experiment and study of the reaction $\nu+d \rightarrow \mu^{-}+p+p_{s}$. Phys. Rev. $D, 16: 3103,1977$.

[58] S. J. Barish et al. Study of Neutrino Interactions in Hydrogen and Deuterium. II. Inelastic Charged Current Reactions. Phys. Rev. D, 19:2521, 1979. 
[59] D.S. Baranov et al. Measurement of the $\nu_{\mu} \mathrm{N}$ total cross section at 2-30 GeV in a skat neutrino experiment. Phys. Lett. B, 81(2):255-257, 1979.

[60] S. Bonetti et al. Study of Quasi-Elastic Reactions of $\nu$ and $\bar{\nu}$ in Gargamelle. Nuovo Cim., A38:260-270, 1977.

[61] T. Kitagaki et al. High-energy quasielastic $\nu_{\mu} n \rightarrow \mu^{-} p$ scattering in deuterium. Phys. Rev. D, 28:436-442, Aug 1983.

[62] S. V. Belikov et al. Quasielastic Neutrino and Antineutrino Scattering: Total CrossSections, Axial-Vector Form-Factor. Z. Phys., A320:625, 1985.

[63] J. P. Berge et al. Total neutrino and antineutrino charged current cross-section measurements in 100, 160, and $200 \mathrm{GeV}$ narrow band beams. Z. Phys., C35:443, 1987.

[64] J. Brunner et al. Quasielastic nucleon and hyperon production by neutrinos and antineutrinos with energies below $30 \mathrm{GeV}$. Z. Phys. C, 45:551-555, 1990.

[65] D.C. Colley et al. Cross Sections for Charged Current $\nu$ and $\bar{\nu}$ Interactions in the Energy Range 10 to $50 \mathrm{GeV}$. Z. Phys. C, 2:187-190, 1979.

[66] S. Ciampolillo et al. Total crosssection for neutrino charged current interactions at 3 $\mathrm{GeV}$ and $9 \mathrm{GeV}$. Phys. Lett. B, 84:281, 1979.

[67] D. MacFarlane et al. Nucleon Structure Functions from High-Energy Neutrino Interactions with Iron and QCD Results. Z. Phys. C, 26:1, 1984.

[68] J. Morfin et al. Total cross sections and nucleon structure functions in the gargamelle SPS neutrino/antineutrino experiment. Phys. Lett. B, 104(3):235-238, 1981.

[69] A. S. Vovenko et al. Sov. J. Nucl. Phys., 30:527, 1979.

[70] G.P. Zeller. Neutrino Cross Sections. PANIC plenary talk, 2011.

[71] N. Armenise et al. Charged current elastic antineutrino interactions in propane. Nuclear Physics B, 152(3-4):365-375, 1979. 
[72] A. E. Asratian et al. Quasielastic Anti-neutrino Interactions In Neon And Total Cross-sections In The Energy Range $10 \mathrm{GeV}$ To $50 \mathrm{GeV}$. Sov. J. Nucl. Phys., 39:392, 1984.

[73] G. Fanourakis et al. Study of low-energy antineutrino interactions on protons. Phys. Rev. D, 21:562-568, Feb 1980.

[74] C. H. Llewellyn Smith. Neutrino Reactions at Accelerator Energies. Phys. Rept., $3: 261,1972$.

[75] T. Katori. PhD thesis, Indiana University, 2008.

[76] H. S. Budd and others. Modeling Quasi-elastic Form Factors for Electron and Neutrino Scattering. arXiv/hep-ex/0308005, 2003.

[77] M. K. Jones et al. $G_{E_{p}} / G_{M_{p}}$ Ratio by Polarization Transfer in $\overrightarrow{e p} \rightarrow e \vec{p}$. Phys. Rev. Lett., 84:1398-1402, Feb 2000.

[78] O. Gayou et al. Measurement of $G_{E_{p}} / G_{M_{p}}$ in $\overrightarrow{e p} \rightarrow e \vec{p}$ to $Q^{2}=5.6 \mathrm{GeV}^{2}$. Phys. Rev. Lett., 88:092301, Feb 2002.

[79] A.F. Krutov and others. Extraction of the neutron charge form-factor $G_{E}^{n}\left(Q^{2}\right)$ from the charge form-factor of deuteron $G_{C}^{d}\left(Q^{2}\right)$. Eur. Phys. J., A16:285-290, 2003.

[80] S. Galster et al. Elastic electron-deuteron scattering and the electric neutron form factor at four-momentum transfers $5 \mathrm{fm}^{-2}<q^{2}<14 \mathrm{fm}^{-2}$. Nuclear Physics B, $32(1): 221-237,1971$.

[81] A. Bodek and Howard Scott others. Extraction of the axial nucleon form-factor from neutrino experiments on deuterium. J. Phys. Conf. Ser., 110:082004, 2008.

[82] L.B. Auerbach et al. Measurements of charged current reactions of muon neutrinos on C-12. Phys. Rev. C, 66:015501, 2002.

[83] V. Lyubushkin et al. A Study of quasi-elastic muon neutrino and antineutrino scattering in the NOMAD experiment. Eur. Phys. J., C63:355-381, 2009. 
[84] R.A. Smith and E.J. Moniz. Neutrino reactions on nuclear targets. Nuclear Physics $B, 43: 605-622,1972$.

[85] A. A. Aguilar-Arevalo et al. Measurement of muon neutrino quasielastic scattering on carbon. Phys. Rev. Lett., 100:032301, 2008.

[86] D. Rein and L. M. Sehgal. Neutrino Excitation of Baryon Resonances and Single Pion Production. Ann. Phys., 133:79, 1981.

[87] D. Rein and L. M. Sehgal. Coherent $\pi^{0}$ production in neutrino Reactions. Nucl. Phys., B223:29, 1983.

[88] D. Rein and L. M. Sehgal. PCAC and the Deficit of Forward Muons in $\pi^{+}$Production by Neutrinos. Phys. Lett. B, 657:207-209, 2007.

[89] D. Rein. Angular Distribution in neutrino induced single pion production processes. Z. Phys., C35:43-64, 1987.

[90] Y. Nakajima. PhD thesis, Kyoto University, 2011.

[91] M. Gluck et al. Dynamical parton distributions revisited. Eur. Phys. J., C5:461-470, 1998.

[92] A. Bodek and U. K. Yang. Modeling neutrino and electron scattering cross sections in the few $\mathrm{GeV}$ region with effective LO PDFs. arXiv/hep-ex/0308007, 2003.

[93] R. Brun et al. GEANT Detector Description and Simulation Tool, 1994.

[94] B.C. Brown et al. Study of Scintillation, Fluorescence, and Scattering in Mineral Oil for the MiniBooNE Neutrino Detector. IEEE Nucl. Sci. Symp. Conf. Rec. 1, pages 652-656, 2004.

[95] C. Zeitnitz and T.A. Gabriel. The GEANT - CALOR interface and benchmark calculations of ZEUS test calorimeters. Nucl. Instrum. Meth., A349:106-111, 1994.

[96] A.A. Aguilar-Arevalo et al. Unexplained Excess of Electron-Like Events From a 1-GeV Neutrino Beam. Phys. Rev. Lett., 102:101802, 2009. 
[97] A. Heikkinen, N. Stepanov, and J. P. Wellisch. Bertini intra-nuclear cascade implementation in Geant4. arXiv:nucl-th/0306008, 2003.

[98] J.L.A. Aunion. PhD thesis, Universidad Autonoma de Barcelona, 2010.

[99] R.B. Patterson et al. The Extended-track reconstruction for MiniBooNE. Nucl. Instrum. Meth., A608:206-224, 2009.

[100] K.B.M. Mahn. PhD thesis, Columbia University, 2009.

[101] A.A. Aguilar-Arevalo et al. Measurement of the neutrino component of an antineutrino beam observed by a nonmagnetized detector. Phys. Rev. D, 84:072005, Oct 2011.

[102] J. Grange. New Results from MiniBooNE Charged-Current Quasi-Elastic AntiNeutrino Data. AIP Conf. Proc., 1405:83-88, 2011.

[103] HARP Collaboration. Paper in preparation.

[104] G. Cheng et al. Measurement of $K^{+}$production cross section by $8 \mathrm{GeV}$ protons using high-energy neutrino interactions in the SciBooNE detector. Phys. Rev. D, 84:012009, Jul 2011.

[105] Y. Nakajima et al. Measurement of inclusive charged current interactions on carbon in a few-GeV neutrino beam. Phys. Rev. D, 83:012005, 2011.

[106] J.E. Amaro et al. Meson-exchange currents and quasielastic antineutrino cross sections in the SuperScaling Approximation. Phys. Rev. Lett., 108:152501, 2012.

[107] A. Bodek et al. Neutrino Quasielastic Scattering on Nuclear Targets: Parametrizing Transverse Enhancement (Meson Exchange Currents). Eur. Phys. J., C71:1726, 2011.

[108] J. Nieves et al. The nucleon axial mass and the MiniBooNE Quasielastic NeutrinoNucleus Scattering problem. Phys. Lett. B, 707:72-75, 2012.

[109] M. Martini and J. others. Unified approach for nucleon knock-out and coherent and incoherent pion production in neutrino interactions with nuclei. Phys. Rev. C, 80:065501, 2009. 
[110] D. Ashery et al. True Absorption and Scattering of pions on Nuclei. Phys. Rev. C, 23:2173-2185, 1981.

[111] M. K. Jones et al. Pion absorption above the $\Delta$ (1232) resonance. Phys. Rev. C, 48:2800-2817, 1993.

[112] R. D. Ransome et al. Pion absorption in heavy nuclei. Phys. Rev. C, 45:509-513, 1992.

[113] Gary J. Feldman and Robert D. Cousins. Unified approach to the classical statistical analysis of small signals. Phys. Rev. D, 57:3873-3889, Apr 1998.

[114] G. Mention, M. Fechner, Th. Lasserre, Th.A. Mueller, D. Lhuillier, et al. The Reactor Antineutrino Anomaly. Phys.Rev., D83:073006, 2011. 


\section{Appendix A}

\section{MiniBooNE and SciBooNE Muon}

\section{Antineutrino Disappearance \\ Publication}

The following appendix contains the paper of this thesis work published in Physical Review D. 
PHYSICAL REVIEW D 86, 052009 (2012)

\section{Dual baseline search for muon antineutrino disappearance at $0.1 \mathrm{eV}^{2}<\Delta m^{2}<100 \mathrm{eV}^{2}$}

G. Cheng, ${ }^{6, a}$ W. Huelsnitz, ${ }^{15, b}$ A. A. Aguilar-Arevalo, ${ }^{18}$ J. L. Alcaraz-Aunion, ${ }^{3}$ S. J. Brice, ${ }^{7}$ B. C. Brown, ${ }^{7}$ L. Bugel, ${ }^{17}$ J. Catala-Perez, ${ }^{24}$ E. D. Church, ${ }^{25}$ J. M. Conrad, ${ }^{17}$ R. Dharmapalan, ${ }^{1}$ Z. Djurcic, ${ }^{2}$ U. Dore,${ }^{21}$ D. A. Finley, ${ }^{7}$ R. Ford, ${ }^{7}$ A. J. Franke, ${ }^{6}$ F. G. Garcia, ${ }^{7}$ G. T. Garvey, ${ }^{15}$ C. Giganti, ${ }^{21, c}$ J. J. Gomez-Cadenas, ${ }^{24}$ J. Grange, ${ }^{8}$ P. Guzowski, ${ }^{10, d}$ A. Hanson, ${ }^{11}$ Y. Hayato, ${ }^{12}$ K. Hiraide,${ }^{14, \mathrm{e}}$ C. Ignarra, ${ }^{17}$ R. Imlay, ${ }^{16}$ R. A. Johnson, ${ }^{4}$ B. J. P. Jones, ${ }^{17}$ G. Jover-Manas, ${ }^{3}$

G. Karagiorgi, ${ }^{6,17}$ T. Katori, ${ }^{11,17}$ Y. K. Kobayashi, ${ }^{23}$ T. Kobilarcik, ${ }^{7}$ H. Kubo, ${ }^{14}$ Y. Kurimoto, ${ }^{14, f}$ W. C. Louis, ${ }^{15}$ P. F. Loverre,${ }^{21}$ L. Ludovici, ${ }^{21}$ K. B. M. Mahn, ${ }^{6, g}$ C. Mariani, ${ }^{6, \mathrm{~h}}$ W. Marsh, ${ }^{7}$ S. Masuike,${ }^{23}$ K. Matsuoka, ${ }^{14}$ V. T. McGary, ${ }^{17}$ W. Metcalf, ${ }^{16}$ G. B. Mills,${ }^{15}$ J. Mirabal, ${ }^{15}$ G. Mitsuka, ${ }^{13, i}$ Y. Miyachi, ${ }^{23, j}$ S. Mizugashira, ${ }^{23}$ C. D. Moore, ${ }^{7}$ J. Mousseau, ${ }^{8}$

Y. Nakajima, ${ }^{14, \mathrm{k}}$ T. Nakaya, ${ }^{14}$ R. Napora,${ }^{20,1}$ P. Nienaber,${ }^{22}$ D. Orme,${ }^{14}$ B. Osmanov, ${ }^{8}$ M. Otani,${ }^{14}$ Z. Pavlovic, ${ }^{15}$

D. Perevalov, ${ }^{7}$ C. C. Polly, ${ }^{7}$ H. Ray, ${ }^{8}$ B. P. Roe, ${ }^{19}$ A. D. Russell, ${ }^{7}$ F. Sanchez,${ }^{3}$ M. H. Shaevitz,${ }^{6}$ T.-A. Shibata, ${ }^{23}$ M. Sorel, ${ }^{24}$ J. Spitz ${ }^{17}$ I. Stancu, ${ }^{1}$ R. J. Stefanski, ${ }^{7}$ H. Takei,${ }^{23, \mathrm{~m}}$ H.-K. Tanaka, ${ }^{17}$ M. Tanaka, ${ }^{9}$ R. Tayloe, ${ }^{11}$ I. J. Taylor, ${ }^{10, n}$ R. J. Tesarek, ${ }^{7}$ Y. Uchida, ${ }^{10}$ R. G. Van de Water, ${ }^{15}$ J. J. Walding, ${ }^{10,0}$ M. O. Wascko, ${ }^{10}$ D. H. White, ${ }^{15}$ H. B. White, ${ }^{7}$ D. A. Wickremasinghe, ${ }^{4}$ M. Yokoyama, ${ }^{14, p}$ G. P. Zeller, ${ }^{7}$ and E. D. Zimmerman ${ }^{5}$

(MiniBooNE and SciBooNE Collaborations)

${ }^{1}$ University of Alabama, Tuscaloosa, Alabama 35487, USA

${ }^{2}$ Argonne National Laboratory, Argonne, Illinois 60439, USA

${ }^{3}$ Institut de Fisica d'Altes Energies, Universitat Autonoma de Barcelona, E-08193 Bellaterra (Barcelona), Spain

${ }^{4}$ University of Cincinnati, Cincinnati, Ohio 45221, USA

${ }^{5}$ University of Colorado, Boulder, Colorado 80309, USA

${ }^{6}$ Columbia University, New York, New York 10027, USA

${ }^{7}$ Fermi National Accelerator Laboratory, Batavia, Illinois 60510, USA

${ }^{8}$ University of Florida, Gainesville, Florida 32611, USA

${ }^{9}$ High Energy Accelerator Research Organization (KEK), Tsukuba, Ibaraki 305-0801, Japan

${ }^{10}$ Imperial College London, London SW7 2AZ, United Kingdom

${ }^{11}$ Indiana University, Bloomington, Indiana 47405, USA

${ }^{12}$ Kamioka Observatory, Institute for Cosmic Ray Research, University of Tokyo, Gifu 506-1205, Japan

${ }^{13}$ Research Center for Cosmic Neutrinos, Institute for Cosmic Ray Research, University of Tokyo, Kashiwa, Chiba 277-8582, Japan ${ }^{14}$ Kyoto University, Kyoto 606-8502, Japan

${ }^{15}$ Los Alamos National Laboratory, Los Alamos, New Mexico 87545, USA

${ }^{16}$ Louisiana State University, Baton Rouge, Louisiana 70803, USA

${ }^{17}$ Massachusetts Institute of Technology, Cambridge, Massachusetts 02139, USA

${ }^{18}$ Instituto de Ciencias Nucleares, Universidad Nacional Autónoma de México, D.F. 04510, México

${ }^{19}$ University of Michigan, Ann Arbor, Michigan 48109, USA

${ }^{20}$ Purdue University Calumet, Hammond, Indiana 46323, USA

\footnotetext{
${ }^{\mathrm{a} C}$ Corresponding author. gcc2113@columbia.edu

${ }^{\mathrm{b}}$ Corresponding author.
} whuelsn@fnal.gov

${ }^{c}$ Present address: DSM/Irfu/SPP, CEA Saclay, F-91191 Gif-sur-Yvette, France.

${ }^{\mathrm{d}}$ Present address: The School of Physics and Astronomy, The University of Manchester, Manchester, M13 9PL, United Kingdom. ${ }^{\text {e}}$ Present address: Kamioka Observatory, Institute for Cosmic Ray Research, University of Tokyo, Gifu 506-1205, Japan.

${ }^{f}$ Present address: High Energy Accelerator Research Organization (KEK), Tsukuba, Ibaraki 305-0801, Japan.

${ }^{\mathrm{g}}$ Present address: TRIUMF, Vancouver, British Columbia, V6T 2A3, Canada.

${ }^{h}$ Present address: Center for Neutrino Physics, Virginia Tech, Blacksburg, VA, USA.

${ }^{\text {i } P r e s e n t ~ a d d r e s s: ~ S o l a r-T e r r e s t r i a l ~ E n v i r o n m e n t ~ L a b o r a t o r y, ~ N a g o y a ~ U n i v e r s i t y, ~ F u r o-c h o, ~ C h i k u s a-k u, ~ N a g o y a, ~ J a p a n . ~}$

${ }^{j}$ Present address: Yamagata University, Yamagata, 990-8560 Japan.

${ }^{k}$ Present address: Lawrence Berkeley National Laboratory, Berkeley, CA 94720, USA.

${ }^{\mathrm{l}}$ Present address: Epic Systems, Inc.

${ }^{m}$ Present address: Kitasato University, Tokyo, 108-8641 Japan.

${ }^{\mathrm{n}}$ Present address: Department of Physics and Astronomy, State University of New York, Stony Brook, New York 11794-3800, USA.

${ }^{\circ}$ Present address: Department of Physics, Royal Holloway, University of London, Egham, TW20 0EX, United Kingdom.

${ }^{\mathrm{p}}$ Present address: Department of Physics, University of Tokyo, Tokyo 113-0033, Japan. 


\section{APPENDIX A. MINIBOONE AND SCIBOONE MUON ANTINEUTRINO DISAPPEARANCE PUBLICATION}

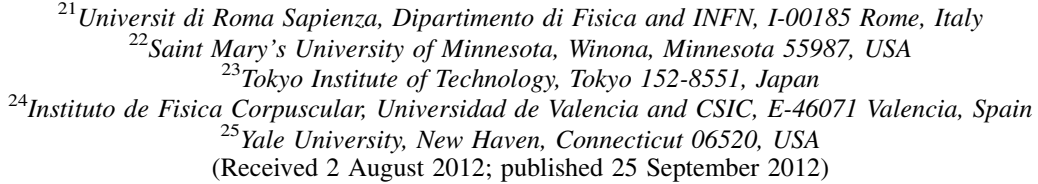

The MiniBooNE and SciBooNE collaborations report the results of a joint search for short baseline disappearance of $\bar{\nu}_{\mu}$ at Fermilab's Booster Neutrino Beamline. The MiniBooNE Cherenkov detector and the SciBooNE tracking detector observe antineutrinos from the same beam, therefore the combined analysis of their data sets serves to partially constrain some of the flux and cross section uncertainties. Uncertainties in the $\nu_{\mu}$ background were constrained by neutrino flux and cross section measurements performed in both detectors. A likelihood ratio method was used to set a $90 \%$ confidence level upper limit on $\bar{\nu}_{\mu}$ disappearance that dramatically improves upon prior limits in the $\Delta m^{2}=0.1-100 \mathrm{eV}^{2}$ region.

\section{INTRODUCTION}

Recently there has been increasing evidence in support of neutrino oscillations in the $\Delta m^{2} \approx 1 \mathrm{eV}^{2}$ region. The LSND [1] experiment observed an excess of $\bar{\nu}_{e}$-like events in a $\bar{\nu}_{\mu}$ beam. MiniBooNE [2-4] has observed an excess of $\nu_{e}$-like and $\bar{\nu}_{e}$-like events, in a $\nu_{\mu}$ beam and $\bar{\nu}_{\mu}$ beam, respectively. Additional evidence for short baseline anomalies with $L / E \approx 1$, where $L$ is the neutrino path length in $\mathrm{km}$ and $E$ the neutrino energy in $\mathrm{GeV}$, includes the deficit of events observed in reactor antineutrino experiments [5] and radioactive source neutrino measurements [6]. If these anomalies are due to neutrino oscillations in the $\Delta m^{2} \approx 1 \mathrm{eV}^{2}$ range, then they could imply the existence of one or more new sterile neutrino species that do not participate in standard weak interactions but mix with the known neutrino flavors through additional mass eigenstates. Observation of $\nu_{\mu}\left(\bar{\nu}_{\mu}\right)$ disappearance in conjunction with $\nu_{e}\left(\bar{\nu}_{e}\right)$ appearance in this $\Delta m^{2}$ range would be a smoking gun for the presence of these sterile neutrinos. Alternatively, constraining $\nu_{\mu}\left(\bar{\nu}_{\mu}\right)$ disappearance can, along with global $\nu_{e}\left(\bar{\nu}_{e}\right)$ disappearance data, constrain the oscillation interpretation of the $\nu_{e}$ $\left(\bar{\nu}_{e}\right)$ appearance signals in LSND and MiniBooNE [7].

Searches for $\nu_{\mu}$ and $\bar{\nu}_{\mu}$ disappearance in MiniBooNE were performed in 2009 [8]. No evidence for disappearance was found. The search for $\nu_{\mu}$ disappearance was recently repeated in MiniBooNE with the inclusion of data from the SciBooNE detector in a joint analysis [9]. Once again, the results were consistent with no $\nu_{\mu}$ disappearance. The analysis presented here is an improved search for $\bar{\nu}_{\mu}$ disappearance using data from MiniBooNE and SciBooNE taken while the Booster Neutrino Beamline (BNB) operated in antineutrino mode.

The Monte Carlo (MC) predictions for both MiniBooNE and SciBooNE were updated to account for recent neutrino flux and cross section measurements made with both experiments. The data from both detectors were then simultaneously fit to a simple two-antineutrino oscillation model. Improved constraints on MC predictions, the inclusion of SciBooNE data, and a MiniBooNE antineutrino data set nearly 3 times larger than what was available for the original $\bar{\nu}_{\mu}$ disappearance analysis, have allowed a $90 \%$ confidence level upper limit to be set that dramatically improves upon prior limits in the $\Delta m^{2}=0.1-100 \mathrm{eV}^{2}$ region, pushing down into the region of parameter space of interest to sterile neutrino models.

This paper is organized as follows. Section II describes the BNB and the MiniBooNE and SciBooNE detectors. Then, the simulation of neutrino interactions with nuclei and subsequent detector responses are described in Sec. III. The event selection and reconstruction for both detectors are described in Sec. IV. The parameters for the MC tuning and its systematic uncertainties are given in Sec. V. Section VI describes the analysis methodology. The results of the analysis are presented in Sec. VII, and the final conclusions are given in Sec. VIII.

\section{BEAM LINE AND EXPERIMENTAL APPARATUS}

MiniBooNE and SciBooNE both use the BNB at Fermilab in Batavia, Illinois. The $8 \mathrm{GeV}$ kinetic energy protons from the booster accelerator strike a 1.7 interaction length beryllium target, which is located inside a focusing horn. The horn is pulsed in time with the beam to produce a toroidal magnetic field that, depending on the polarity setting, will either focus $\pi^{-} / K^{-}$and defocus $\pi^{+} / K^{+}$or vice versa. These mesons then pass through a $60 \mathrm{~cm}$ long

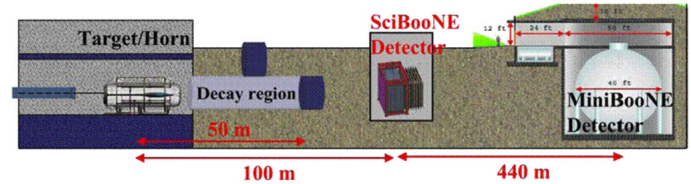

FIG. 1 (color online). Schematic view of the BNB from the beryllium target and magnetic horn to the SciBooNE and MiniBooNE detectors. 
DUAL BASELINE SEARCH FOR MUON ANTINEUTRINO ...

PHYSICAL REVIEW D 86, 052009 (2012)

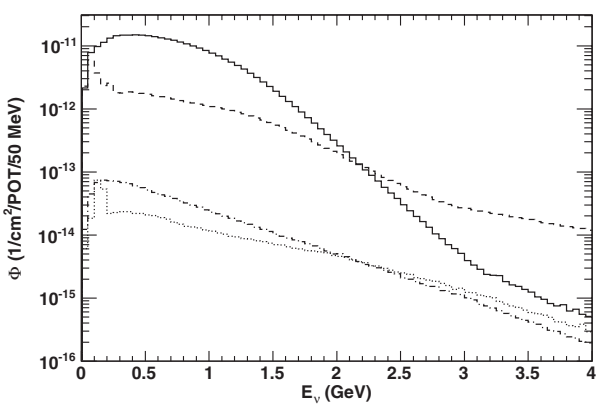

(a) MiniBooNE antineutrino mode flux

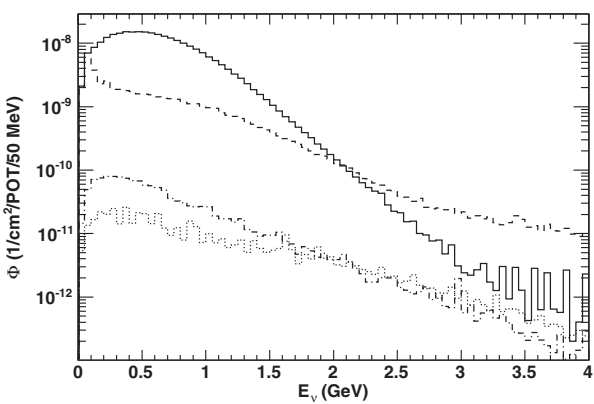

(b) SciBooNE antineutrino mode flux

FIG. 2. The neutrino and antineutrino flux prediction as a function of true neutrino (antineutrino) energy, in antineutrino mode at the MiniBooNE and SciBooNE detectors. The $\bar{\nu}_{\mu}$ flux is represented by the solid line, the $\nu_{\mu}$ flux is represented by the dashed line, the $\bar{\nu}_{e}$ flux is represented by the dot-dashed line, and the $\nu_{e}$ flux is represented by the dotted line.

collimator and decay in flight along a $50 \mathrm{~m}$ long tunnel. A schematic view of the BNB from the beryllium target to both detectors is shown in Fig. 1.

The resulting neutrino beam will have an enhanced flux of either muon neutrinos (neutrino mode) or muon antineutrinos (antineutrino mode). In antineutrino mode beam running, the flux of antineutrinos in the beam will be referred to as the right-sign (RS) flux and the flux of neutrinos in the beam will be referred to as the wrong-sign (WS) flux. These two designations are used because antineutrinos are the signal in this analysis and neutrinos are an intrinsic background. Figure 2 shows the neutrino and antineutrino flux prediction in antineutrino mode at both the MiniBooNE and SciBooNE detectors. Details on the beam line and flux predictions are given in Ref. [10].

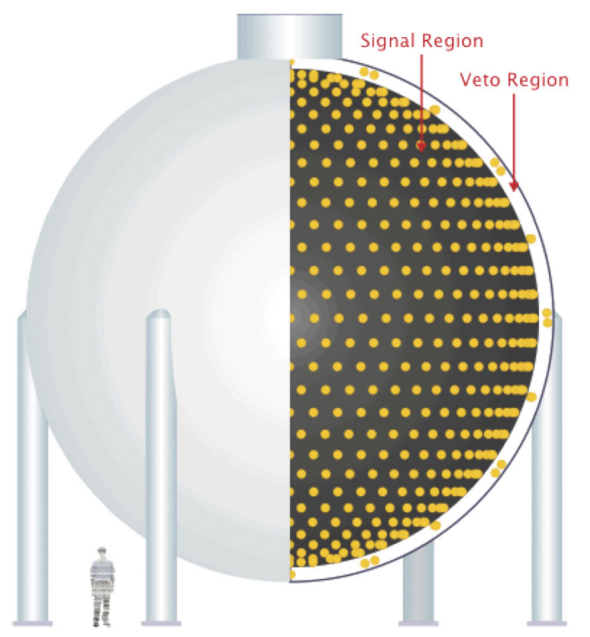

FIG. 3 (color online). Schematic view of the MiniBooNE detector.
The MiniBooNE detector [11] is located $541 \mathrm{~m}$ downstream of the antineutrino production target and consists of a spherical $12.2 \mathrm{~m}$ diameter tank containing 800 tons of mineral oil $\left(\mathrm{CH}_{2}\right)$, beneath at least $3 \mathrm{~m}$ of earth overburden. The fiducial volume is a sphere $10 \mathrm{~m}$ in diameter, with a fiducial mass of 450 tons. The detector is instrumented with 1280 8-inch photomultiplier tubes (PMTs) in the active region, and 2408 -inch PMTs in an outer, veto region. Events are reconstructed based on timing and charge information mostly from Cherenkov radiation. A schematic of the MiniBooNE detector is shown in Fig. 3.

The SciBooNE detector [12] is located $100 \mathrm{~m}$ downstream of the target. SciBooNE is a discrete tracking detector comprised of three subdetectors (in order from upstream to downstream): a fully active and finely segmented scintillator tracker (SciBar), an electromagnetic calorimeter (EC), and a muon range detector (MRD). The SciBar subdetector [13] consists of 14336 extruded polystyrene $\left(\mathrm{C}_{8} \mathrm{H}_{8}\right)$ strips arranged vertically and horizontally to construct a $3 \times 3 \times 1.7 \mathrm{~m}^{3}$ volume. Each scintillator strip is read out by a wavelength shifting fiber attached to a 64-channel multianode PMT (MA-PMT).

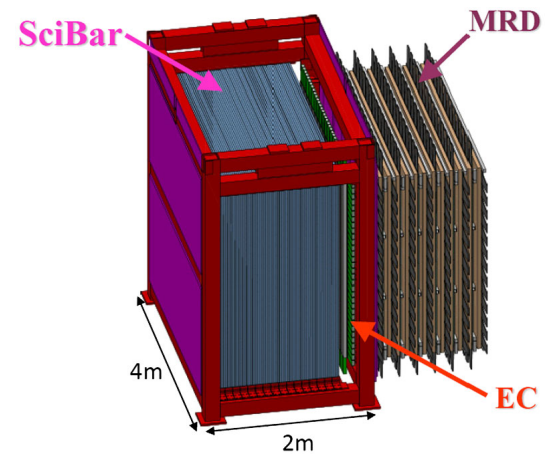

FIG. 4 (color online). Schematic view of the SciBooNE detector. 
G. CHENG et al.

The 15 ton SciBar subdetector (10.6 ton fiducial volume) provides the primary interaction target. The EC subdetector is a two plane (vertical and horizontal) "spaghetti"-type calorimeter; 64 modules made of $1 \mathrm{~mm}$ scintillating fibers embedded in lead foil are bundled and read out at both ends by PMTs. The MRD subdetector, designed to measure muon momentum, is made from 12 iron plates, each $5 \mathrm{~cm}$ thick, sandwiched between 13 alternating horizontal and vertical scintillator planes of thickness $6 \mathrm{~mm}$ that are read out via 362 individual 2-inch PMTs. A schematic of the SciBooNE detector is shown in Fig. 4.

\section{MONTE CARLO SIMULATION}

Simulation of the neutrino and antineutrino flux, neutrino and antineutrino interactions in the detector, and detector response has been discussed in detail in previous publications for MiniBooNE [14,15] and SciBooNE [16]. Calculation of the neutrino and antineutrino flux at the detector is done with a GEANT4-based model [17] that is constrained by external measurements $[10,18]$ and accounts for proton transport to the target, $p$-Be interactions in the target, meson production, focusing by the magnetic horn, meson propagation and decay, and neutrino and antineutrino propagation to the detectors.

Neutrino and antineutrino interactions in both detectors are simulated using the NUANCE [19] event generator. Bound nucleons are described by the relativistic Fermi gas (RFG) model [20]. The MiniBooNE detector response is simulated using GEANT3 [21], which takes the final-state particles emerging from a nucleus and propagates them through the detector. The GEANT3 code was modified to include a custom model for light propagation in the detector [22] and to use GCALOR [23] for pion absorption and charge exchange in the detector medium. SciBooNE uses GEANT4 [24] to simulate the interactions of hadronic particles with detector materials.

\section{EVENT SELECTION AND RECONSTRUCTION}

MiniBooNE data from a total of $1.01 \times 10^{21}$ protons on target (POT) operation in antineutrino mode, from July 2006 up through April 2012, are included in the analysis. Data from SciBooNE antineutrino mode operation from June 2007 through August 2008 are included, comprising a total of $1.53 \times 10^{20}$ POT for the SciBooNE contribution.

MiniBooNE event selection and reconstruction is essentially identical to that used for a previous neutrino mode $\nu_{\mu}$ cross section measurement [14]. Events with only a single $\mu^{+}$in the detector are selected. Event selection cuts are based on the beam timing, fiducial volume, observation of two correlated events (the muon and its decay electron), and the likelihood of the fit to the muon hypothesis. These cuts are designed to reject incoming particles (i.e. muons from cosmic rays or from neutrino and antineutrino interactions in the surrounding material), ensure that the event is contained
PHYSICAL REVIEW D 86, 052009 (2012)

within the detector, and ensure correct event classification as well as accurate muon energy estimation. The capture of $\mu^{-}$ resulting from initial $\nu_{\mu}$ charged current quasielastic (CCQE) interaction events is simulated in the $\mathrm{MC}$ and these specific events are not selected. In antineutrino mode, a sizable fraction of the events (roughly 20\%) are due to $\nu_{\mu}$ interactions. MiniBooNE cannot distinguish between $\nu_{\mu}$ and $\bar{\nu}_{\mu}$ events on an event-by-event basis, so $\mu^{-} \mathrm{s}$ from $\nu_{\mu}$ interactions are an irreducible background.

For SciBooNE, the event selection and reconstruction is nearly identical to the previous inclusive charged current measurement [16]. Two-dimensional SciBar tracks are reconstructed using a cellular automaton algorithm [25] from SciBar hits. Three-dimensional SciBar tracks are then reconstructed based on the timing and end point positions of the two-dimensional SciBar tracks. Two-dimensional tracks in the MRD are independently reconstructed using hits in the MRD that are clustered within a $50 \mathrm{~ns}$ timing window. Three-dimensional tracks in the MRD are reconstructed by matching the timing of the two-dimensional projections. If the downstream edge of a SciBar track lies in the last two layers of SciBar, a search for a matching track or hits in the MRD is performed. The upstream edge of the MRD track is required to be on either one of the first two layers of the MRD, and to be within $30 \mathrm{~cm}$ of the projected entry point of the SciBar track into the MRD (a more detailed description of the track reconstruction can be found in Ref. [12]).

To select $\mu^{+}$events, the highest momentum track per event in the beam on-time window is required to have $p_{\mu}>0.25 \mathrm{GeV} / c$ to reduce the number of neutral current (NC) events. The energy loss of the track in SciBar must be consistent with a muon hypothesis, and must originate within the 10.6 ton SciBar fiducial volume. These muon candidate tracks are further categorized as SciBar-stopped or MRD-stopped. SciBar-stopped events have the downstream end point of the muon candidate track contained in the SciBar fiducial volume. MRD-stopped events have the muon candidate track being a SciBar track matched to MRD hits or to an MRD track with a downstream end point that does not exit the back or sides of the MRD. Both SciBar-stopped and MRD-stopped events are used in the analysis. SciBooNE has no overburden so cosmic backgrounds must be subtracted. For cosmic background estimation, the same muon selection criteria are applied to a beam-off time window that is 5 times longer than the beam-on window. This event rate is scaled and subtracted from the beam-on data.

The selected events include $\bar{\nu}_{\mu}$ and $\nu_{\mu}$ interactions on carbon and hydrogen in the detectors. The reconstructed antineutrino energy is based on the assumption that the interaction is always a $\bar{\nu}_{\mu}$ CCQE interaction with a proton at rest in carbon: $\bar{\nu}_{\mu}+p \rightarrow \mu+n$. Hence, it is a function of the measured energy and direction of the outgoing muon. The equation for reconstructed energy is 


\section{APPENDIX A. MINIBOONE AND SCIBOONE MUON ANTINEUTRINO DISAPPEARANCE PUBLICATION}

DUAL BASELINE SEARCH FOR MUON ANTINEUTRINO ...

$$
E_{\nu}^{\mathrm{QE}}=\frac{M_{n}^{2}-\left(M_{p}-E_{B}\right)^{2}-M_{\mu}^{2}+2\left(M_{p}-E_{B}\right) E_{\mu}}{2\left(M_{p}-E_{B}-E_{\mu}+P_{\mu} \cos \theta_{\mu}\right)},
$$

where $M_{n}$ and $M_{p}$ are the mass of the neutron and proton, $M_{\mu}, E_{\mu}, P_{\mu}$, and $\theta_{\mu}$ are the mass, energy, momentum, and direction of the outgoing muon, and $E_{B}$ is the binding energy ( $30 \mathrm{MeV}$ for protons in carbon). Equation (1) is applied to all selected events in data and $\mathrm{MC}$, even though a sizable fraction of the events are not CCQE [i.e. charged current single $\pi(\mathrm{CC} 1 \pi)$, charged current multipion (CC multipion), or NC events misidentified as CCQE events]. The impact of the CCQE reconstruction assumption, which leads to reduced accuracy in reconstructed energy for nonCCQE events, is accounted for in MC, which also includes these selected non-CCQE events. MiniBooNE has an estimated resolution for reconstructed energy of $8.3 \%$ for CCQE events and $13.9 \%$ for all events. SciBooNE has an estimated reconstructed energy resolution of $9.6 \%$ for CCQE events and $24.6 \%$ for all events.

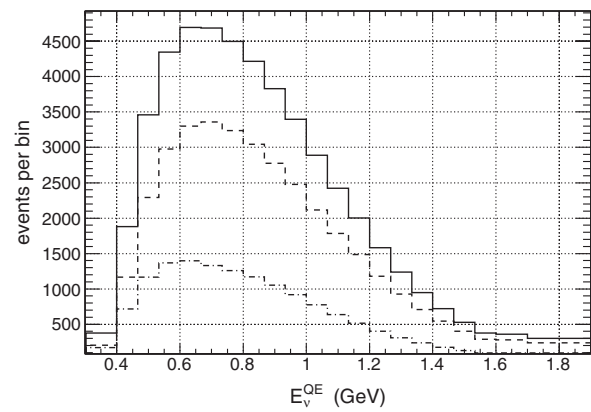

(a) MiniBooNE RS Events
PHYSICAL REVIEW D 86, 052009 (2012)

MiniBooNE and SciBooNE data and MC are put in 21-bin histograms of $E_{\nu}^{\mathrm{QE}}$. The binning goes from $300 \mathrm{MeV}$ to $1.9 \mathrm{GeV}$, with individual bin widths as follows: bin 1, $100 \mathrm{MeV}$; bins 2-19, $66.7 \mathrm{MeV}$; bin 20, $100 \mathrm{MeV}$; bin 21, $200 \mathrm{MeV}$. The first and last two bins are wider to ensure adequate event statistics in data and MC.

Figure 5 shows the predicted event distributions in MiniBooNE for reconstructed antineutrino and neutrino energy, for events on hydrogen and carbon nuclei. Figure 6 shows the predictions for MiniBooNE's reconstructed antineutrino and neutrino energy distributions by interaction type: $\mathrm{CCQE}, \mathrm{CC} 1 \pi$, and all other interaction types $(\mathrm{CC}$ multipion and $\mathrm{NC}$ ). Table I shows the MC predictions for the selected MiniBooNE events by neutrino and interaction type. $\nu_{e}$ and $\bar{\nu}_{e}$ contamination is negligible.

The following plots show several properties of the selected SciBooNE events, as predicted by simulation. Figure 7 shows the reconstructed antineutrino and neutrino energy distributions for events on hydrogen and carbon

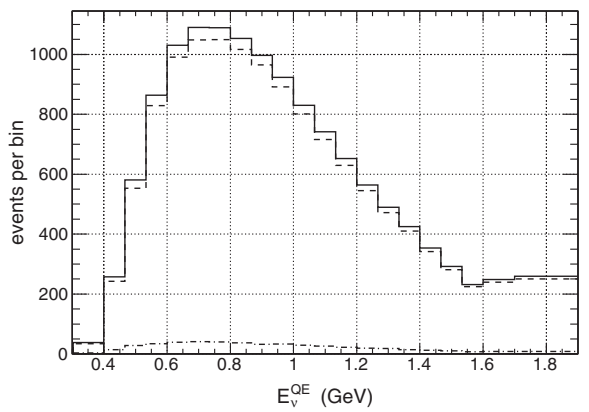

(b) MiniBooNE WS Events

FIG. 5. Reconstructed antineutrino and neutrino energy $\left(E_{\nu}^{\mathrm{QE}}\right)$ distributions for selected RS and WS MiniBooNE events on different target types (hydrogen or carbon) from MiniBooNE MC. Total events are represented by the solid line, events with interaction on carbon are represented by the dashed line, and events with interaction on hydrogen are represented by the dot-dashed line.

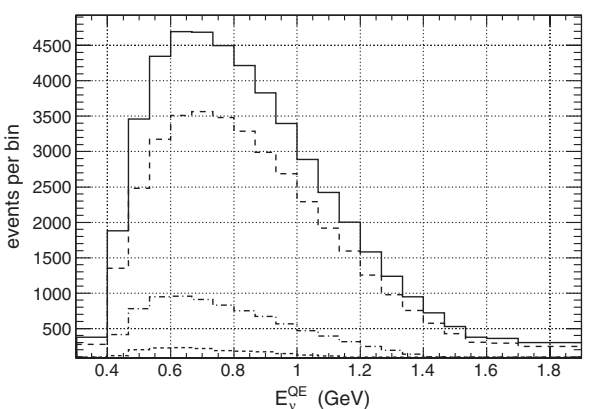

(a) MiniBooNE RS Events

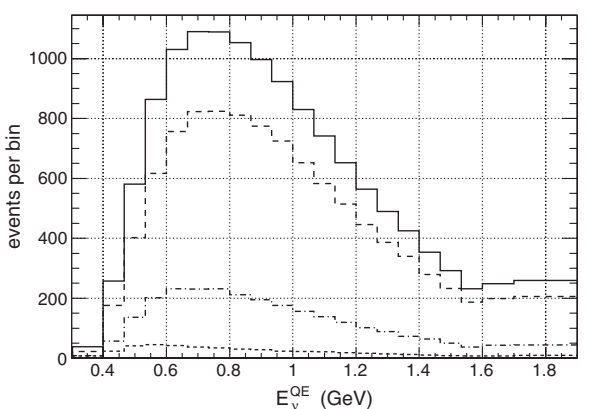

(b) MiniBooNE WS Events

FIG. 6. Reconstructed antineutrino and neutrino energy $\left(E_{\nu}^{\mathrm{QE}}\right)$ distributions for selected RS and WS MiniBooNE events for different interaction types (CCQE, CC1 $\pi$, other) from MiniBooNE MC. Total events are represented by the solid line, CCQE interaction events are represented by the dashed line, $\mathrm{CC} 1 \pi$ interaction events are represented by the dot-dashed line, and all other interaction (CC multi- $\pi$ or NC) events are represented by the short-dashed line. 
G. CHENG et al.

TABLE I. MC predictions for the number of selected events by neutrino and interaction type in both MiniBooNE and SciBooNE.

\begin{tabular}{lcccc}
\hline \hline & \multicolumn{2}{c}{ MiniBooNE } & \multicolumn{2}{c}{ SciBooNE } \\
\hline Interaction type & $\bar{\nu}$ events & $\nu$ events & $\bar{\nu}$ events & $\nu$ events \\
CCQE & 37428 & 9955 & 4619 & 1359 \\
$\mathrm{CC} 1 \pi$ & 8961 & 2593 & 1735 & 1006 \\
$\mathrm{CC}$ multi- $\pi$ or NC & 2364 & 460 & 959 & 610 \\
\hline \hline
\end{tabular}

nuclei. Figure 8 shows the reconstructed antineutrino and neutrino energy distribution by interaction type: CCQE, $\mathrm{CC} 1 \pi$, and other (CC multipion and NC). Table I shows the MC predictions for the selected SciBooNE events by neutrino and interaction type. The data set is estimated to contain an additional 811 events from cosmic ray muons.
PHYSICAL REVIEW D 86, 052009 (2012)

The difference in shape between the SciBooNE RS (WS) and MiniBooNE RS (WS) energy distributions is mainly due to different event selection criteria between MiniBooNE and SciBooNE. MiniBooNE selects for CCQE interaction events and SciBooNE selects for all $\mathrm{CC}$ interaction events so the SciBooNE sample has a larger percentage of non-CCQE interaction events. Since the antineutrino energy reconstruction is based on a CCQE interaction assumption, there are more SciBooNE events with a larger discrepancy between true antineutrino energy and reconstructed antineutrino energy than in MiniBooNE, leading to shape differences. Differences in selection efficiency, antineutrino flux at the detector locations, and background rejection between MiniBooNE and SciBooNE also contribute to the shape differences.

Figure 9 shows the distribution of the combined antineutrino and neutrino propagation distances, from production

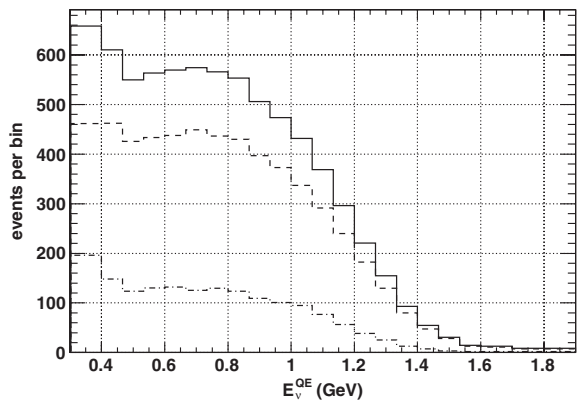

(a) SciBooNE RS Events

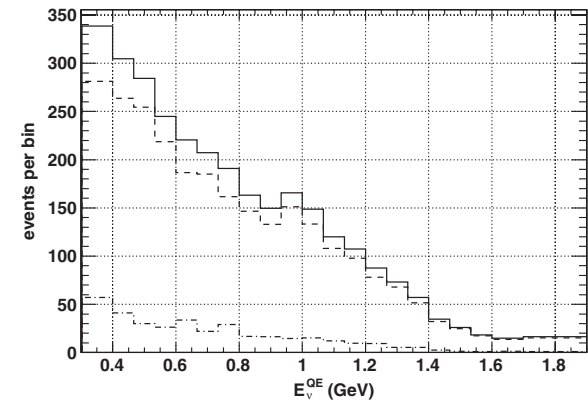

(b) SciBooNE WS Events

FIG. 7. Reconstructed antineutrino and neutrino energy $\left(E_{\nu}^{\mathrm{QE}}\right)$ distributions for selected RS and WS SciBooNE events on different target types (hydrogen or carbon) from SciBooNE MC. Total events are represented by the solid line, events with interaction on carbon are represented by the dashed line, and events with interaction on hydrogen are represented by the dot-dashed line.

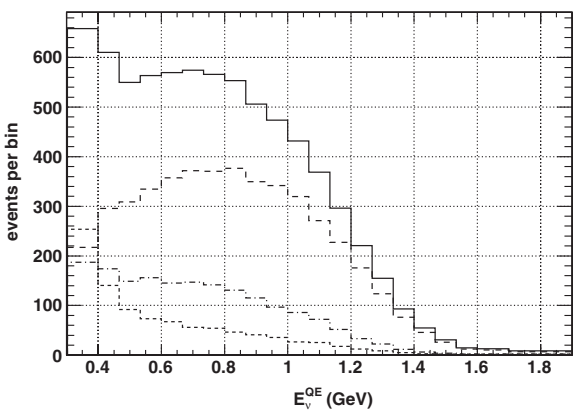

(a) SciBooNE RS Events

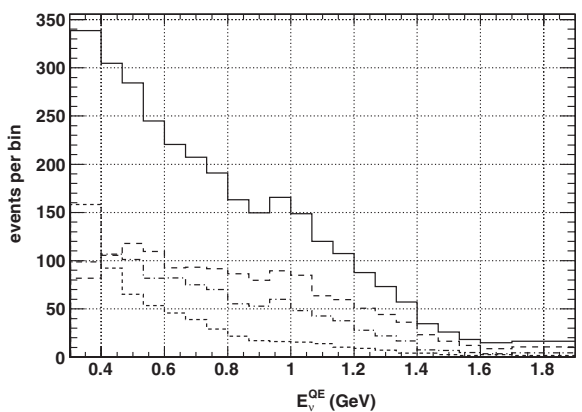

(b) SciBooNE WS Events

FIG. 8. Reconstructed antineutrino and neutrino energy $\left(E_{\nu}^{\mathrm{QE}}\right)$ distributions for selected RS and WS SciBooNE events for different interaction types (CCQE, $\mathrm{CC} 1 \pi$, other) from SciBooNE MC. Total events are represented by the solid line, CCQE interaction events are represented by the dashed line, $\mathrm{CC} 1 \pi$ interaction events are represented by the dot-dashed line, and all other interaction (CC multi- $\pi$ or NC) events are represented by the short-dashed line. 


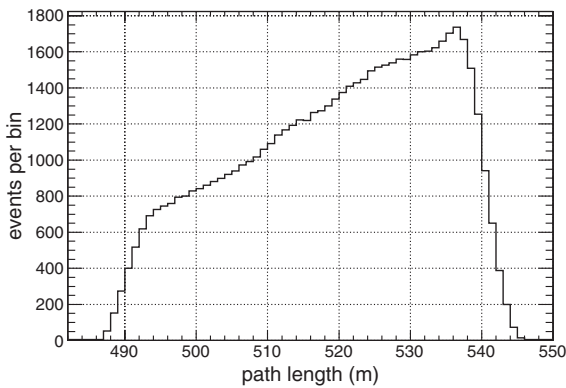

(a) MiniBooNE (anti)neutrino path lengths

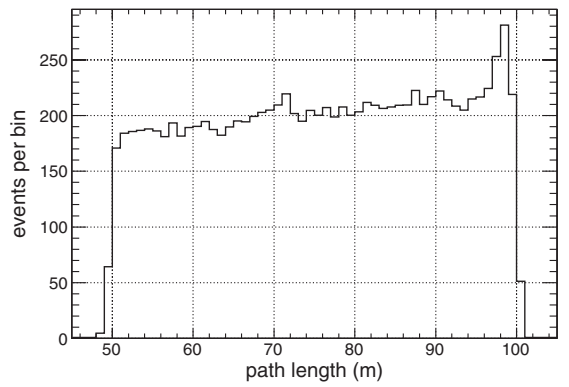

(b) SciBooNE (anti)neutrino path lengths

FIG. 9. Antineutrino and neutrino path lengths for MiniBooNE and SciBooNE events from point of production to interaction in detector, as predicted by the MC simulation.

in the decay tunnel to interaction in SciBooNE or MiniBooNE.

\section{SYSTEMATIC UNCERTAINTIES}

Beam and cross section uncertainties are calculated for both MiniBooNE and SciBooNE using the multisim method [26]. In this procedure, groups of correlated simulation parameters associated with beam production and cross section modeling uncertainties are sampled according to their covariance matrices. The parameters for each source of uncertainty $\left(\pi^{ \pm}, K^{+}\right.$, etc.) are sampled 1000 times to obtain sufficient statistics. Each MC event in MiniBooNE and SciBooNE is reweighted based on these varied parameters forming 1000 new MC predictions of the $E_{\nu}^{\mathrm{QE}}$ distribution in both detectors. Covariance matrices, in bins of $E_{\nu}^{\mathrm{QE}}$, are then computed for each source of uncertainty by comparing these 1000 new MC predictions to the default MC prediction. The procedure takes care of the correlation of beam production and cross section uncertainties between MiniBooNE and SciBooNE. Cross section and nuclear model uncertainties for $\nu$ and $\bar{\nu}$ events are treated as uncorrelated due to the poor understanding of differences between $\nu$ and $\bar{\nu}$ interactions in nuclear modeling. Some detector specific uncertainties are calculated using the unisim method [26], where uncorrelated detector specific uncertainties are varied up or down by 1 standard deviation.

\section{A. Beam uncertainties}

Uncertainties in the delivery of the primary proton beam to the beryllium target, the primary beam optics, secondary hadron production in proton-beryllium interactions, hadronic interactions in the target and horn, and the horn magnetic field, are included in the beam multisims. Uncertainties in the magnetic field horn current, skin effect of the horn, and secondary nucleon and pion interactions in the $\mathrm{Be}$ target and $\mathrm{Al}$ horn are obtained from previous MiniBooNE analyses [10].

The normalization of the neutrino component in the antineutrino beam was adjusted based on direct measurements in MiniBooNE $[15,27]$. The beam fraction of $\nu_{\mu}$ in the antineutrino beam was determined using three methods: a pure data sample of $\nu_{\mu}$ events from $\mathrm{CC} 1 \pi$ interactions, differences in Michel electron rates between final state $\mu^{-}$and $\mu^{+}$from $\nu_{\mu}$ and $\bar{\nu}_{\mu}$ interactions, respectively, due to $\mu^{-}$capture on carbon, and angular distribution differences between final state $\mu^{-}$and $\mu^{+}$from $\nu_{\mu}$ and $\bar{\nu}_{\mu}$ interactions, respectively. Averaging these three methods, the $\pi^{+}$production in the beam MC was scaled by a factor of 0.78 and given a $12.8 \%$ normalization uncertainty. Uncertainties on the production of $\pi^{-}$from the initial $p$-Be interaction are calculated using spline fits to data from the HARP experiment [10]. An updated $K^{+}$ production simulation with reduced uncertainties for the initial $p$-Be interaction is used. This update is based on a new Feynman scaling fit [28] to recent SciBooNE measurements [29]. The $K^{0}$ production uncertainties for the initial $p$-Be interaction are from the Sanford-Wang parametrization covariance matrix [10]. $K^{-}$production is estimated using the MARS hadronic interaction package [30] due to the scarcity of production measurements in the relevant kinematic regions. $K^{-}$production cross section uncertainties from the initial $p$-Be interaction are given a conservative $100 \%$ normalization uncertainty.

\section{B. Cross section uncertainties}

CCQE cross sections on carbon are calculated assuming an RFG model with parameters $M_{A}$ (axial mass) $=1.35$ and $\kappa$ (Pauli blocking factor $)=1.007$. An additional correction, as a function of $Q^{2}$, is applied to background $\mathrm{CC} 1 \pi$ interaction events in MC [14]. The uncertainties in $M_{A}$ and $\kappa$ for CCQE events on carbon are based on the statistical uncertainties of the MiniBooNE neutrino mode measurement [14], to avoid double counting systematic uncertainties accounted for in this analysis as detailed in this section.

Since the purpose of the $Q^{2}$ correction in the MiniBooNE neutrino mode measurement [14] is to match 


\section{APPENDIX A. MINIBOONE AND SCIBOONE MUON ANTINEUTRINO DISAPPEARANCE PUBLICATION}

\section{G. CHENG et al.}

the background $\mathrm{CC} 1 \pi$ interaction events in $\mathrm{MC}$ to a selected data sample comprising mainly of $\mathrm{CC} 1 \pi$ interaction events, there is no uncertainty placed on $M_{A}$ for $\nu_{\mu} \mathrm{CC} 1 \pi$ interaction events. However, for $\bar{\nu}_{\mu} \mathrm{CC} 1 \pi$ interaction events, the $M_{A}$-resonant and coherent $1 \pi$ uncertainties are not constrained by the MiniBooNE neutrino mode measurement and are not reduced. The values and uncertainties of $M_{A}$ for CC coherent $\pi$ interactions, $M_{A}$ for multi- $\pi$ interactions, Fermi surface momentum $\left(p_{F}\right)$, and $\mathrm{NC}$ axial vector isoscalar contribution $(\Delta \mathrm{s})$ are identical to previous MiniBooNE and SciBooNE measurements $[9,16]$. The uncertainties for pion absorption, pion inelastic scattering, and pionless $\Delta$ decay in the target nucleus ( $\pm 25 \%, \pm 30 \%$, and $\pm 100 \%$, respectively) are treated in the same way as in a previous measurement [9], however they are treated as uncorrelated between MiniBooNE and SciBooNE (unlike all other cross section uncertainties). Both the $\nu$ and $\bar{\nu} M_{A}$ values and their uncertainties for quasielastic interactions on hydrogen are based on the latest deuterium measurements [31].

Additional systematic uncertainties are added to account for limitations of the RFG model. Such limitations include the absence of processes such as meson exchange currents and multinucleon knockout events [32-35]. A 10\% normalization uncertainty is assigned to both $\nu$ and $\bar{\nu} \mathrm{CCQE}$
PHYSICAL REVIEW D 86, 052009 (2012)

interactions on carbon to cover the difference between data and prediction in the MiniBooNE $\nu_{\mu}$ CCQE measurement. An additional $40 \%$ normalization uncertainty is placed on $\bar{\nu}$ CCQE interactions on carbon to cover the discrepancy between the RFG model prediction for $\bar{\nu}$ and recent nuclear models [32-35]. An additional 10\% normalization uncertainty is added to non-CCQE $\bar{\nu}$ interactions on carbon to account for the limitations of the RFG model for those type of events.

The full list of beam and cross section parameters for MC simulation and its associated systematic uncertainties are shown in Table II.

\section{Detector uncertainties}

Uncertainties associated with the MiniBooNE detector include light propagation, attenuation, and scattering in the detector as well as PMT response. The optical model for light propagation in the detector [22] uses 35 parameters for properties such as refractive index, attenuation length, scintillation strength, etc. These parameters are tuned to non-MiniBooNE measurements as well as MiniBooNE internal data. Over 100 separate MC data sets were created based on variations in these parameters. In a manner similar to the multisim method, these results were used to compute the optical model error matrix in bins of

TABLE II. Summary of beam and cross section parameters for MC simulation with its associated systematic uncertainties.

\begin{tabular}{lc}
\hline \hline Beam & Uncertainty \\
\hline$\pi^{+}$production in antineutrino beam (from WS neutrino background) & $12.8 \%$ normalization uncertainty [15] \\
$\pi^{-}$production from $p$-Be interaction & Spline fit to HARP data \\
$K^{+}$production from $p$-Be interaction & Table IX in Ref. [28] \\
$K^{0}$ production from $p$-Be interaction & Table IX in Ref. [10] \\
$K^{-}$production from $p$-Be interaction & $100 \%$ normalization uncertainty \\
Nucleon and pion interaction in Be/Al & Table XIII in Ref. [10] \\
Horn current & $\pm 1 \mathrm{kA}$ \\
Horn skin effect & Horn skin depth, $\pm 1.4 \mathrm{~mm}$ \\
\hline Cross Sections & Uncertainty \\
\hline CCQE $M_{A}$ on carbon target & $1.35 \pm 0.07 \mathrm{GeV}$ \\
$\kappa$ & $1.007 \pm 0.005$ \\
CCQE $M_{A}$ on hydrogen target & $1.014 \pm 0.014 \mathrm{GeV}$ \\
CC resonant $\pi M_{A}$ & $1.1 \pm 0.275 \mathrm{GeV}$ \\
CC coherent $\pi M_{A}$ & $1.03 \pm 0.275 \mathrm{GeV}$ \\
CC multi $\pi M_{A}$ & $1.3 \pm 0.52 \mathrm{GeV}$ \\
$E_{B}$ & $\pm 9 \mathrm{MeV}$ \\
$p_{F}$ & $220 \pm 30 \mathrm{MeV} / \mathrm{c}$ \\
$\Delta \mathrm{SCQE}$ on carbon & $0.0 \pm 0.1$ \\
CCQE on carbon $\left(\bar{\nu}_{\mu}\right)$ only & $\pm 10 \%$ norm error \\
Non-CCQE on carbon $\left(\bar{\nu}_{\mu}\right)$ only & $\pm 40 \%$ norm error \\
$\pi$ absorption in nucleus & $\pm 10 \%$ norm error \\
$\pi$ inelastic scattering & $25 \%$ \\
$\pi$-less $\Delta$ decay & $30 \%$ \\
\hline \hline
\end{tabular}

${ }^{\mathrm{a}}$ This uncertainty is not applied to $\nu_{\mu} \mathrm{CC} 1 \pi$ events that are $Q^{2}$ corrected. 


\section{APPENDIX A. MINIBOONE AND SCIBOONE MUON ANTINEUTRINO DISAPPEARANCE PUBLICATION}

\section{DUAL BASELINE SEARCH FOR MUON ANTINEUTRINO ...}

reconstructed antineutrino energy. To estimate the impact of uncertainties in PMT response, independent MC data sets based on variations in the discriminator threshold, or the PMT charge-time correlations, were created and compared to default MC. Based on comparisons with external data [36-38] and the output of GCALOR, an uncertainty of $35 \%$ is assigned to pion absorption and $50 \%$ is assigned to charge exchange in the detector medium. This is distinct from the uncertainty on pion absorption and charge exchange inside the nucleus.

Uncertainties associated with the SciBooNE detector include uncertainties in the muon energy loss in the scintillator and iron, light attenuation in the wavelength shifting fibers, and PMT response; see Ref. [16]. The crosstalk of the MA-PMT was measured to be $3.15 \%$ for adjacent channels with an absolute error of $0.4 \%$ [12]. The single photoelectron resolution of the MA-PMT is set to $50 \%$ in the simulation, and the absolute error is estimated to be $\pm 20 \%$. Birk's constant for quenching in the SciBar scintillator was measured to be $0.0208 \pm 0.0023 \mathrm{~cm} / \mathrm{MeV}$ [12]. The conversion factors for analog-to-digital converter counts to photoelectrons were measured for all 14336 MAPMT channels in SciBar. The measurement uncertainty was at the $20 \%$ level. The threshold for hits to be used in SciBar track reconstruction is 2.5 photoelectrons; this threshold is varied by $\pm 20 \%$ to evaluate the systematic error for SciBar track reconstruction. The time-to-digital converter dead time is set to $55 \mathrm{~ns}$ in the MC simulation, with the error estimated to be $\pm 20 \mathrm{~ns}$ [39].

The reconstruction uncertainties consist of antineutrino energy reconstruction uncertainties and muon track misidentification uncertainties. For antineutrino energy reconstruction uncertainties, the densities of SciBar, EC, and MRD are varied independently within their measured uncertainties of $\pm 3 \%, \pm 10 \%$, and $\pm 3 \%$, respectively. Misidentified muons stem mainly from proton tracks created through NC interactions, which are given a conservative $\pm 20 \%$ normalization uncertainty. A conservative $\pm 20 \%$ normalization uncertainty is applied for the MC simulated background of neutrino and antineutrino events initially interacting outside the SciBooNE detector that pass the selection criteria. A conservative $\pm 20 \%$ normalization uncertainty is applied for the MC simulated background of neutrino and antineutrino events initially interacting in the EC/MRD detector that pass the selection criteria.

\section{Error matrix}

All of the MiniBooNE uncertainties, the SciBooNE uncertainties, and the correlations between them are expressed in the total error matrix, $M$, a $42 \times 42$ covariance matrix in MiniBooNE and SciBooNE reconstructed antineutrino energy bins defined as

$$
M=\left(\begin{array}{ll}
M^{\mathrm{MB}-\mathrm{SB}} & M^{\mathrm{SB}-\mathrm{SB}} \\
M^{\mathrm{MB}-\mathrm{MB}} & M^{\mathrm{SB}-\mathrm{MB}}
\end{array}\right),
$$

PHYSICAL REVIEW D 86, 052009 (2012)

where

$$
\begin{aligned}
M_{i, j}^{\mathrm{X}}= & \hat{M}_{i, j ;(\mathrm{RS}, \mathrm{RS})}^{\mathrm{X}} N_{i}^{\mathrm{YRS}} N_{j}^{\mathrm{ZRS}} \\
& +\hat{M}_{i, j ;(\mathrm{WS}, \mathrm{WS})}^{\mathrm{X}} N_{i}^{\mathrm{Y} \mathrm{WS}} N_{j}^{\mathrm{ZWS}} \\
& +\hat{M}_{i, j ;(\mathrm{RS}, \mathrm{WS})}^{\mathrm{X})} N_{i}^{\mathrm{YRS}} N_{j}^{\mathrm{ZWS}} \\
& +\hat{M}_{i, j ;(\mathrm{WS}, \mathrm{RS})}^{\mathrm{X})} N_{i}^{\mathrm{Y} \mathrm{WS}} N_{j}^{\mathrm{ZRS}} \\
& +M_{i, j}^{\mathrm{X} \text { stat }}
\end{aligned}
$$

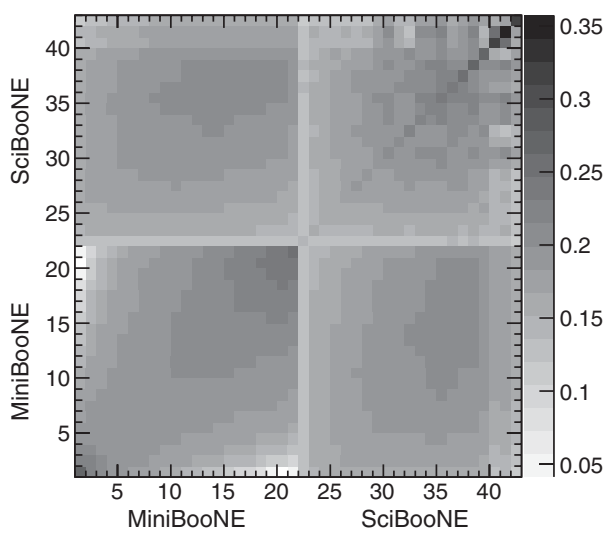

FIG. 10. Bin-wise square root of the total (statistical and systematic errors combined) fractional error matrix $\sqrt{\hat{M}_{i j}}=$ $\sqrt{M_{i j}} / \sqrt{N_{i} N_{j}}$, where $M_{i j}$ is the total error matrix and $N_{i}\left(N_{j}\right)$ is the MC prediction for reconstructed antineutrino energy bin $i$ (j). Bins 1 through 21 are MiniBooNE, bins 22 through 42 are SciBooNE.

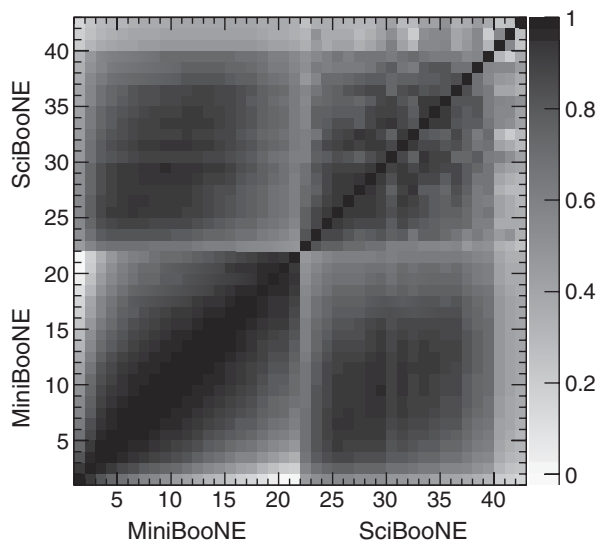

FIG. 11. Correlation coefficients of the total (statistical and systematic errors combined) error matrix $\left(\rho_{i j}=M_{i j} /\left(\sigma_{i i} \sigma_{j j}\right)\right)$. Bins 1 through 21 are MiniBooNE, bins 22 through 42 are SciBooNE. No bins are anticorrelated. 


\section{APPENDIX A. MINIBOONE AND SCIBOONE MUON ANTINEUTRINO

G. CHENG et al.

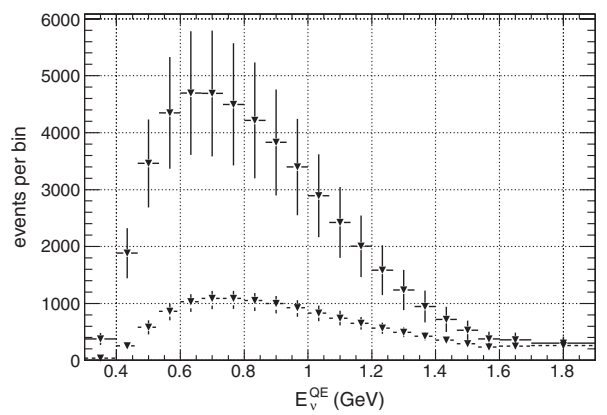

(a) MiniBooNE RS and WS events with systematic
PHYSICAL REVIEW D 86, 052009 (2012)

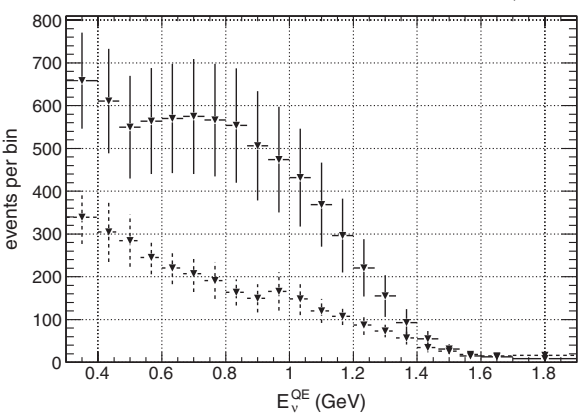

(b) SciBooNE RS and WS events with systematics

FIG. 12. RS (solid line) and WS (dashed line) contributions to the default MC prediction for MiniBooNE and SciBooNE reconstructed antineutrino and neutrino energy $\left(E_{\nu}^{\mathrm{QE}}\right)$ distributions. Error bars are the systematic uncertainties from the diagonals of the error matrix $\left(\sigma_{i i}=\sqrt{M_{i i}}\right)$ and do not account for correlations.

are the bin to bin covariance elements of the full error matrix. $\mathrm{X}$ denotes the type of correlation with $\mathrm{Y}$ and $\mathrm{Z}$ denoting the type of bins (either MiniBooNE or SciBooNE) associated with X. For MiniBooNE to MiniBooNE correlations, $\mathrm{X}=$ $\mathrm{MB}-\mathrm{MB}, \quad \mathrm{Y}=\mathrm{MB}, \mathrm{Z}=\mathrm{MB}$. For SciBooNE to SciBooNE correlations, $\mathrm{X}=\mathrm{SB}-\mathrm{SB}, \mathrm{Y}=\mathrm{SB}, \mathrm{Z}=\mathrm{SB}$. For MiniBooNE to SciBooNE correlations, $X=M B-S B$, $\mathrm{Y}=\mathrm{MB}, \mathrm{Z}=\mathrm{SB}$. For SciBooNE to MiniBooNE correlations, $\mathrm{X}=\mathrm{SB}-\mathrm{MB}, \mathrm{Y}=\mathrm{SB}, \mathrm{Z}=\mathrm{MB} . N_{i}^{\mathrm{YRS}}\left(N_{j}^{\mathrm{ZRS}}\right)$ and $N_{i}^{\mathrm{YWS}}\left(N_{j}^{\mathrm{ZWS}}\right)$ are the number of RS and WS events for bin type $\mathrm{Y}$ (bin type $\mathrm{Z}$ ) in reconstructed antineutrino energy bin $i$ (bin $j$ ), respectively. $\hat{M}_{i, j ;(\mathrm{RS}, \mathrm{RS})}^{\mathrm{X}}$ are the elements of the RS to RS correlated fractional error matrix for correlation type $\mathrm{X}$ defined as

$$
\hat{M}_{i, j ;(\mathrm{RS}, \mathrm{RS})}^{\mathrm{X})}=\frac{M_{i, j ;(\mathrm{RS}, \mathrm{RS})}^{\mathrm{X}}}{N_{i}^{\mathrm{YRS}} N_{j}^{\mathrm{ZRS}}},
$$

where $M_{i, j ;(\mathrm{RS}, \mathrm{RS})}^{\mathrm{X}}$ is the full RS to RS reconstructed antineutrino energy bin covariance for correlation type $\mathrm{X}$. $\hat{M}_{i, j ;(\mathrm{WS}, \mathrm{WS})}^{\mathrm{X}}, \hat{M}_{i, j ;(\mathrm{RS}, \mathrm{WS})}^{\mathrm{X}}$, and $\hat{M}_{i, j ;(\mathrm{WS}, \mathrm{RS})}^{\mathrm{X}}$ are similarly defined fractional error matrices for correlation type $\mathrm{X}$ with different RS and WS correlations. $M^{\mathrm{X} \text { stat }}$ is the statistical covariance matrix in reconstructed antineutrino energy bins for correlation type $\mathrm{X}$ (only SB - SB and MB - MB have nonzero elements).

The decomposition and reconstruction of the full error matrix $M$ to and from the fractional error matrices allows the error matrix to be updated based on different MC predictions, as a function of the oscillation parameters in the physics parameter space.

For illustrative purposes, Fig. 10 shows the square roots of the elements of the total fractional error matrix, $\sqrt{\hat{M}_{i j}}=$ $\sqrt{M_{i j}} / \sqrt{N_{i} N_{j}}$, where $M_{i j}$ are the elements of the total error matrix and $N_{i}\left(N_{j}\right)$ is the MC prediction for reconstructed antineutrino energy bin $i(j)$. Figure 11 shows the correlation coefficients of the total error matrix in reconstructed antineutrino energy bins.
Figure 12 shows the MiniBooNE and SciBooNE default $\mathrm{MC} E_{\nu}^{\mathrm{QE}}$ predictions for RS and WS events with error bars corresponding to the $\sqrt{M_{i i}}$ values of the error matrix diagonal elements.

\section{ANALYSIS METHODOLOGY}

Oscillation predictions are based on a two-antineutrino oscillation model, where the oscillation survival probability for a $\bar{\nu}_{\mu}$ in the beam is given by

$$
P\left(\bar{\nu}_{\mu} \rightarrow \bar{\nu}_{x}\right)=1-\sin ^{2} 2 \theta \sin ^{2} \frac{1.27 \Delta m^{2} L}{E} .
$$

$L$ is the path length in kilometers, $E$ is the antineutrino energy in $\mathrm{GeV}, \theta$ is the mixing angle, and $\Delta m^{2}$ is the difference in the squares of the masses of two different mass eigenstates.

The $\chi^{2}$ statistic is formed,

$$
\chi^{2}=\sum_{i, j=1}^{42}\left(D_{i}-N_{i}\right)\left(M^{-1}\right)_{i j}\left(D_{j}-N_{j}\right),
$$

where $\left(M^{-1}\right)_{i j}$ is the $i j$ th element of the inverse of the error matrix $M$, the covariance matrix in MiniBooNE and SciBooNE $E_{\nu}^{\mathrm{QE}}$ bins described in Eq. (2). $D_{i}\left(D_{j}\right)$ is the data count in bin $i(j)$ and $N_{i}\left(N_{j}\right)$ is the MC prediction for bin $i(j)$, in MiniBooNE and SciBooNE $E_{\nu}^{\mathrm{QE}}$ bins. $N_{i}$ is the sum of neutrino (WS) and antineutrino (RS) events in the $i$ th bin:

$$
N_{i}=N_{i}^{\mathrm{RS}}\left(\Delta m^{2}, \sin ^{2} 2 \theta\right)+N_{i}^{\mathrm{WS}} .
$$

As shown in Eq. (7), only the predicted RS event rate depends on the oscillation parameters, $\Delta m^{2}$ and $\sin ^{2} 2 \theta$, for this twoantineutrino oscillation model. The WS flux is assumed to not oscillate. The index runs from 1 to 42 (21 MiniBooNE $E_{\nu}^{\mathrm{QE}}$ bins and $21 \mathrm{SciBooNE} E_{\nu}^{\mathrm{QE}}$ bins). For the physics analysis fitting, a $\Delta \chi^{2}$ test statistic is used as defined by 
DUAL BASELINE SEARCH FOR MUON ANTINEUTRINO ...

PHYSICAL REVIEW D 86, 052009 (2012)

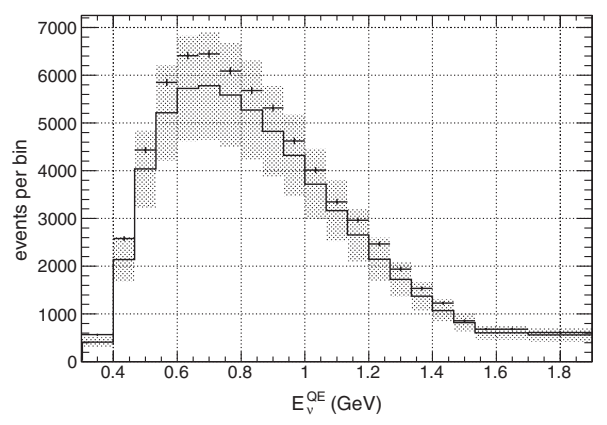

(a) MiniBooNE

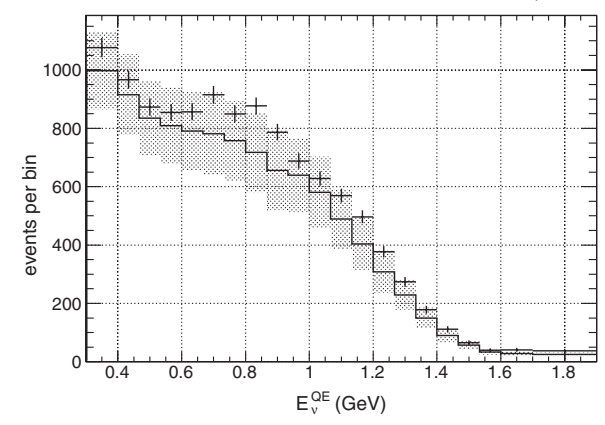

(b) SciBooNE

FIG. 13. Reconstructed antineutrino energy $\left(E_{\nu}^{\mathrm{QE}}\right)$ distribution for data events, compared to Monte Carlo predictions, for MiniBooNE and SciBooNE. Vertical error bars on data are statistical uncertainty. Shaded error band around simulation is the systematic uncertainty computed as the square roots of the diagonals of the total error matrix.

$$
\Delta \chi^{2}=\chi^{2}\left(N\left(\theta_{\text {phys }}\right), M\left(\theta_{\text {phys }}\right)\right)-\chi^{2}\left(N\left(\theta_{\mathrm{BF}}\right), M\left(\theta_{\mathrm{BF}}\right)\right)
$$

where $\theta_{\mathrm{BF}}$ refers to the oscillation parameters at the best fit point and $\theta_{\text {phys }}$ refers to the oscillation parameters at a given test point.

The method of Feldman and Cousins [40] is used to determine the $\Delta \chi^{2}$ value at each point that corresponds to a certain confidence level of acceptance or rejection. To obtain the $90 \%$ confidence level exclusion region for $\bar{\nu}_{\mu}$ disappearance, a $\Delta \chi^{2}$ distribution is formed for each point $\theta_{\text {phys }}$ in parameter space using many iterations of generated fake data at that $\theta_{\text {phys }}$. The $\Delta \chi^{2}$ value from actual data at each $\theta_{\text {phys }}$ is then compared to the fake data $\Delta \chi^{2}$ distribution at each $\theta_{\text {phys }}$. If the $\Delta \chi^{2}$ value from actual data is larger than $90 \%$ of the all the fake data $\Delta \chi^{2}$ values at a $\theta_{\text {phys }}$ point, then the $\theta_{\text {phys }}$ point in parameter space is excluded at $90 \%$ confidence level. The aggregation of all the excluded $90 \%$ confidence level $\theta_{\text {phys }}$ points forms the $90 \%$ confidence level exclusion region.

TABLE III. Observed event counts for each MiniBooNE and SciBooNE data bin, MC predictions, and uncertainty.

\begin{tabular}{lccccccc}
\hline \hline Bin range $(\mathrm{MeV})$ & MB data & MB MC & MB error \pm & SB data $^{a}$ & SB cosmic & SB MC & SB error \pm \\
\hline $300-400$ & 565 & 413.5 & 111.0 & 1077.0 & 21.0 & 997.3 & 136.8 \\
$400-467$ & 2577 & 2139.2 & 464.8 & 966.8 & 89.2 & 915.6 & 141.9 \\
$467-533$ & 4433 & 4039.9 & 802.2 & 872.8 & 85.2 & 834.4 & 132.1 \\
$533-600$ & 5849 & 5211.0 & 1005.7 & 854.4 & 72.6 & 809.4 & 132.2 \\
$600-667$ & 6411 & 5725.6 & 1108.7 & 856.8 & 59.2 & 790.6 & 137.3 \\
$667-733$ & 6445 & 5778.3 & 1130.3 & 915.0 & 51.0 & 781.9 & 144.3 \\
$733-800$ & 6090 & 5586.8 & 1096.9 & 849.8 & 52.2 & 757.3 & 139.5 \\
$800-867$ & 5678 & 5268.3 & 1044.8 & 876.6 & 43.4 & 717.1 & 138.8 \\
$867-933$ & 5314 & 4826.2 & 951.8 & 787.0 & 39.0 & 655.8 & 138.0 \\
$933-1000$ & 4624 & 4319.6 & 865.1 & 688.0 & 35.0 & 639.7 & 129.6 \\
$1000-1067$ & 4015 & 3720.3 & 747.2 & 628.0 & 29.0 & 580.2 & 125.4 \\
$1067-1133$ & 3349 & 3163.6 & 642.1 & 569.6 & 28.4 & 488.7 & 105.8 \\
$1133-1200$ & 2965 & 2655.9 & 554.3 & 496.6 & 21.4 & 403.9 & 92.2 \\
$1200-1267$ & 2464 & 2147.2 & 453.0 & 377.0 & 23.0 & 308.4 & 74.6 \\
$1267-1333$ & 1937 & 1726.4 & 367.8 & 273.6 & 22.4 & 228.4 & 53.6 \\
$1333-1400$ & 1534 & 1372.0 & 297.9 & 178.6 & 18.4 & 150.0 & 37.2 \\
$1400-1467$ & 1227 & 1073.3 & 238.1 & 111.2 & 18.8 & 89.4 & 23.9 \\
$1467-1533$ & 859 & 820.5 & 187.7 & 65.4 & 17.6 & 57.1 & 15.0 \\
$1533-1600$ & 679 & 607.2 & 145.8 & 39.0 & 17.0 & 33.1 & 10.4 \\
$1600-1700$ & 684 & 607.2 & 149.1 & 40.8 & 28.2 & 27.6 & 9.9 \\
$1700-1900$ & 610 & 560.1 & 144.5 & 37.6 & 39.4 & 24.8 & 7.8 \\
\hline \hline
\end{tabular}

${ }^{\mathrm{a}}$ The SB data has its SB cosmic data background removed. 


\section{G. CHENG et al.}

The full error matrix is used to create the fake data for the Feldman and Cousins tests. First, a Cholesky decomposition is performed on the error matrix $M$ :

$$
M=L L^{*},
$$

where $L$ is a lower triangular matrix and $L^{*}$ is the conjugate transpose of $L$. Then, a vector $u$ is created, where each of the $n$ elements, 42 in total, of $u$ are drawn from a Gaussian distribution with mean 0 and variance 1 . A fluctuated fake data histogram is given by

$$
N_{\text {fake }}=N_{\text {default }}\left(\theta_{\text {phys }}\right)+L u,
$$

where $N_{\text {default }}$ is the default Monte Carlo prediction assuming an oscillation signal with oscillation parameters at point $\theta_{\text {phys }}$.

\section{RESULTS}

Figure 13 shows the observed event distributions, in reconstructed antineutrino energy, for MiniBooNE and SciBooNE. The systematic uncertainty shown for the MC predictions was computed as just the square roots of the diagonals of the total error matrix without correlations.

Table III lists the event counts in each bin, for data and for MC predictions. The listed uncertainties are based on the square roots of the diagonals of the total error matrix without correlations. (The reported SciBooNE data has

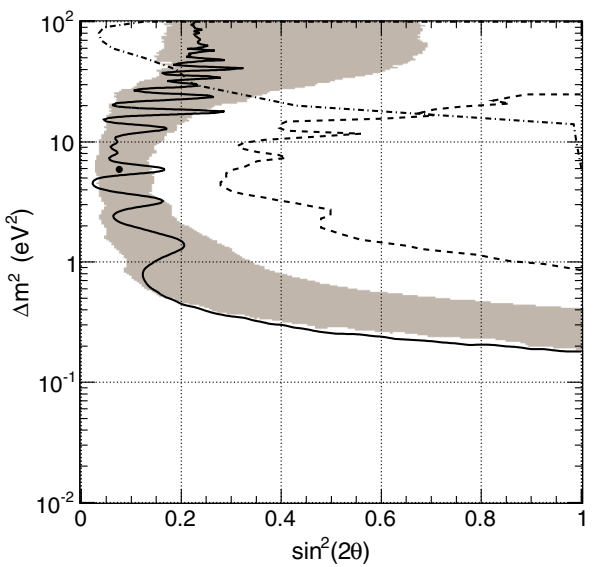

FIG. 14 (color online). 90\% C.L. exclusion region (solid line) and best fit point for the MiniBooNE-only $\bar{\nu}_{\mu}$ disappearance analysis. Also shown is the $90 \%$ C.L. result from the 2009 MiniBooNE disappearance analysis [8] (dashed line) and the Chicago-Columbia-Fermilab-Rochester (CCFR) experiment [41] (dot-dashed line). The expected 90\% C.L. sensitivity band from fake data studies is also shown (shaded region); $1 \sigma(68 \%)$ of fake data tests, where the fake data had statistical and systematic fluctuations but no oscillation signal, had $90 \%$ C.L. limit curves in this shaded region.
PHYSICAL REVIEW D 86, 052009 (2012)

fractional counts due to the manner in which the cosmic ray background is subtracted.)

A MiniBooNE-only disappearance analysis is included to give a sense of what the sensitivity would be without the inclusion of SciBooNE data. Figure 14 shows the 90\% C.L. exclusion region and best fit point for the MiniBooNEonly $\bar{\nu}_{\mu}$ disappearance analysis, completed using the same methodology as the joint disappearance analysis except with the exclusion of SciBooNE data, SciBooNE MC

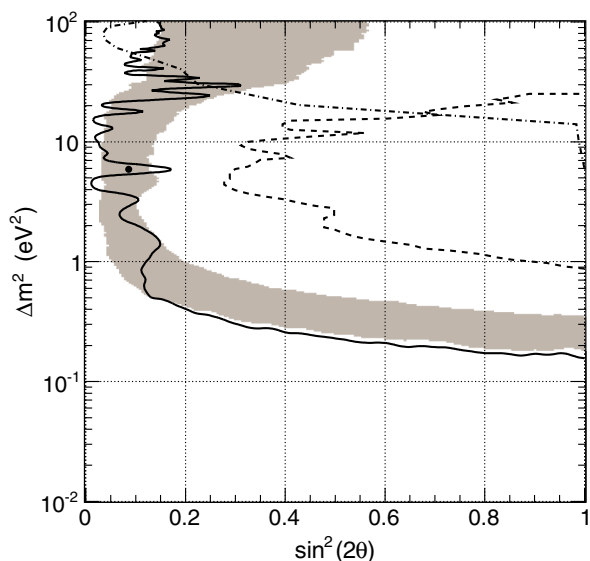

(a) Linear scale on $\mathrm{x}$-axis.

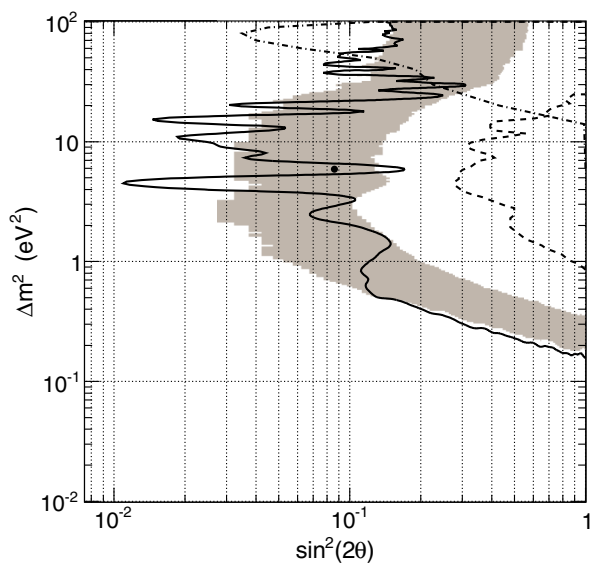

(b) Log scale on $x$-axis.

FIG. 15 (color online). 90\% C.L. exclusion region (solid line) and best fit point for the joint MiniBooNE, SciBooNE $\bar{\nu}_{\mu}$ disappearance analysis. Also shown is the $90 \%$ C.L. result from the 2009 MiniBooNE disappearance analysis [8] (dashed line) and the CCFR experiment [41] (dot-dashed line). The expected $90 \%$ C.L. sensitivity band from fake data studies is also shown (shaded region); $1 \sigma(68 \%)$ of fake data tests, where the fake data had statistical and systematic fluctuations but no oscillation signal, had 90\% C.L. limit curves in this shaded region. 


\section{APPENDIX A. MINIBOONE AND SCIBOONE MUON ANTINEUTRINO DISAPPEARANCE PUBLICATION}

DUAL BASELINE SEARCH FOR MUON ANTINEUTRINO ...

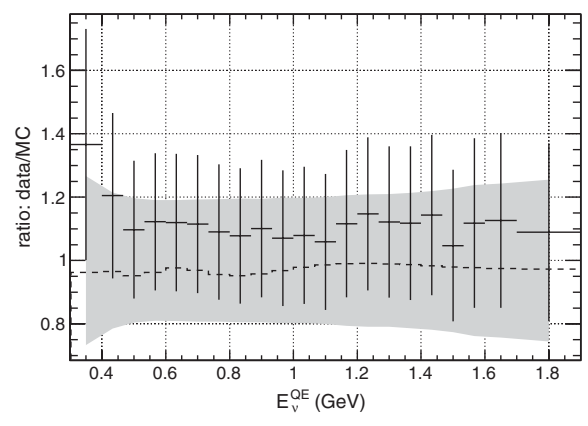

(a) MiniBooNE
PHYSICAL REVIEW D 86, 052009 (2012)

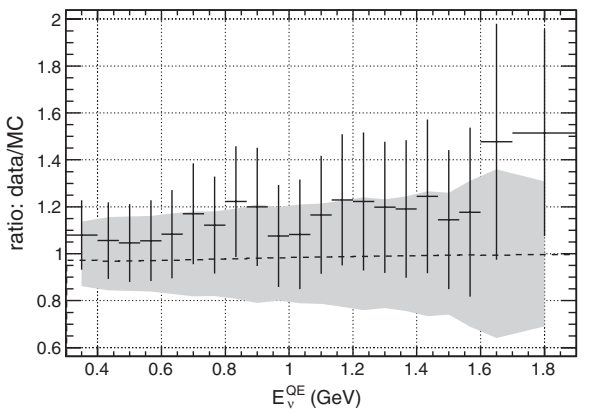

(b) SciBooNE

FIG. 16. The ratio, with error bars, between data and default $\mathrm{MC}$ as a function of reconstructed antineutrino energy $\left(E_{\nu}^{\mathrm{QE}}\right)$. The ratio of best fit signal MC to default MC is also shown (dashed line). The best fit results from the joint analysis were used to generate the signal MC. The shaded regions are the $1 \sigma$ band from fake data with statistical and systematic fluctuations but no oscillation signal.

prediction, and SciBooNE error matrix uncertainties in the $\chi^{2}$ statistic. The best fit point is $\Delta m^{2}=5.9 \mathrm{eV}^{2}, \sin ^{2} 2 \theta=$ 0.076 . At the best fit point, $\chi^{2}=25.7$ (probability $12.4 \%$ ). For the null oscillation hypothesis, $\chi^{2}=28.3$ (probability $13.7 \%$ ). With $\Delta \chi^{2}=2.6$, the null oscillation hypothesis is excluded at $52.4 \%$ C.L.

Figure 15 shows the $\bar{\nu}_{\mu}$ disappearance limit for the joint disappearance analysis. For $\Delta m^{2}=1 \mathrm{eV}^{2}$ and $\Delta m^{2}=$ $10 \mathrm{eV}^{2}$, the $90 \%$ C.L. limit for $\sin ^{2} 2 \theta$ are at 0.121 and 0.024 , respectively. At $\sin ^{2} 2 \theta=1$, the $90 \%$ C.L. limit for $\Delta m^{2}$ is $0.156 \mathrm{eV}^{2}$ The best fit point from the joint analysis is $\Delta m^{2}=5.9 \mathrm{eV}^{2}, \sin ^{2} 2 \theta=0.086$. At the best fit point, $\chi^{2}=40.0$ (probability $47.1 \%$ ). For the null oscillation hypothesis, $\chi^{2}=43.5$ (probability $41.2 \%$ ). With $\Delta \chi^{2}=3.5$, the null oscillation hypothesis is excluded at $81.9 \%$ C.L.

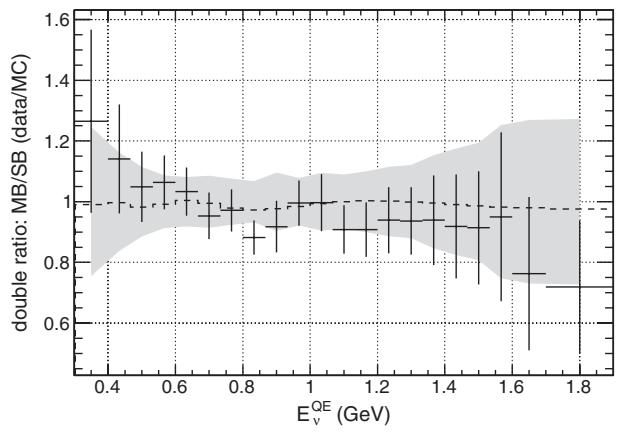

FIG. 17. The double ratio [Eq. (11)], with error bars, as a function of reconstructed antineutrino energy $\left(E_{\nu}^{\mathrm{QE}}\right)$. Some of the MiniBooNE and SciBooNE uncertainties cancel in this double ratio. The double ratio where the MiniBooNE and SciBooNE signal MC based on the best fit results from the joint analysis are used in placed of data is also shown (dashed line). The shaded region is the $1 \sigma$ band from fake data with statistical and systematic fluctuations but no oscillation signal.
All probabilities in both the MiniBooNE-only and joint disappearance analyses are based on fake data studies.

Figure 16 shows the data to MC ratios for MiniBooNE and SciBooNE, as well as how the best fit signal modifies the MC predictions. From these ratio plots, it can be seen how the best fit signal improves the shape agreement between data and MC. Figure 17 shows the double ratio

$$
\frac{\left(\frac{\text { MiniBooNE data }}{\text { MiniBooNE default MC }}\right)}{\left(\frac{\text { SciBooNE data }}{\text { SciBooNE default MC }}\right)} \text {. }
$$

In Fig. 17, any common normalization difference is removed and the expected result is a value of one. The double ratio result agrees well with the expectation except where statistics are small.

\section{CONCLUSIONS}

An improved search for $\bar{\nu}_{\mu}$ disappearance using a twodetector combined MiniBooNE/SciBooNE analysis has been performed. Previous flux and cross section measurements, as well as an increased data set, have enabled a substantial improvement in the sensitivity to $\bar{\nu}_{\mu}$ disappearance. The results are consistent with no short baseline disappearance of $\bar{\nu}_{\mu}$ and we have dramatically improved on the excluded regions of the oscillation parameter space. MiniBooNE and SciBooNE have pushed the limit on short baseline disappearance of $\bar{\nu}_{\mu}$ down to roughly $10 \%$, the region of interest for sterile neutrino models.

\section{ACKNOWLEDGMENTS}

We wish to acknowledge the support of Fermilab, the U.S. Department of Energy, and the National Science Foundation in the construction, operation, and data analysis for the MiniBooNE and SciBooNE experiments. The SciBooNE detector was mainly constructed and operated 


\section{APPENDIX A. MINIBOONE AND SCIBOONE MUON ANTINEUTRINO DISAPPEARANCE PUBLICATION}

G. CHENG et al.

by the budget of Japan-U.S. Cooperative Science Program. We acknowledge the support of MEXT and JSPS (Japan) with the Japan/U.S. Cooperation Program. We also acknowledge the Los Alamos National Laboratory for LDRD funding. We acknowledge the Physics Department at Chonnam National University, Dongshin University, and Seoul National University for the loan of parts used in SciBar and the help in the assembly of SciBar. We wish to thank the Physics Departments at the University of Rochester and Kansas State University for the loan of Hamamatsu PMTs used in the MRD. We gratefully
PHYSICAL REVIEW D 86, 052009 (2012)

acknowledge the support of grants and contracts from the INFN (Italy), the Ministry of Science and Innovation and CSIC (Spain), and the STFC (U.K.). We acknowledge the support by MEXT and JSPS with the Grant-in-Aid for Scientific Research A 19204026, Young Scientists S 20674004, Young Scientists B 18740145, Scientific Research on Priority Areas "New Developments of Flavor Physics," the global COE program "The Next Generation of Physics, Spun from Universality and Emergence," and the Japan-U.S. Cooperative Science Program between JSPS and NSF.
[1] A. Aguilar et al. (LSND Collaboration), Phys. Rev. D 64, 112007 (2001).

[2] A. A. Aguilar-Arevalo et al. (MiniBooNE Collaboration), Phys. Rev. Lett. 98, 231801 (2007).

[3] A. Aguilar-Arevalo et al. (MiniBooNE Collaboration), Phys. Rev. Lett. 102, 101802 (2009).

[4] A. A. Aguilar-Arevalo et al. (MiniBooNE Collaboration), Phys. Rev. Lett. 105, 181801 (2010).

[5] G. Mention, M. Fechner, Th. Lasserre, Th. Mueller, D. Lhuillier, M. Cribier, and A. Letourneau, Phys. Rev. D 83, 073006 (2011)

[6] C. Giunti and M. Laveder, Phys. Rev. C 83, 065504 (2011).

[7] A. A. Aguilar-Arevalo et al. (MiniBooNE Collaboration), arXiv: 1207.4809 .

[8] A. Aguilar-Arevalo et al. (MiniBooNE Collaboration), Phys. Rev. Lett. 103, 061802 (2009).

[9] K. Mahn et al. (SciBooNE and MiniBooNE Collaborations), Phys. Rev. D 85, 032007 (2012).

[10] A. Aguilar-Arevalo et al. (MiniBooNE Collaboration), Phys. Rev. D 79, 072002 (2009).

[11] A. Aguilar-Arevalo et al. (MiniBooNE Collaboration), Nucl. Instrum. Methods Phys. Res., Sect. A 599, 28 (2009).

[12] K. Hiraide et al. (SciBooNE Collaboration), Phys. Rev. D 78, 112004 (2008)

[13] K. Nitta et al., Nucl. Instrum. Methods Phys. Res., Sect. A 535, 147 (2004).

[14] A. Aguilar-Arevalo et al. (MiniBooNE Collaboration), Phys. Rev. D 81, 092005 (2010).

[15] A. Aguilar-Arevalo et al. (MiniBooNE Collaboration), Phys. Rev. D 84, 072005 (2011).

[16] Y. Nakajima et al. (SciBooNE Collaboration), Phys. Rev. D 83, 012005 (2011).

[17] S. Agostinelli et al. (GEANT4 Collaboration), Nucl. Instrum. Methods Phys. Res., Sect. A 506, 250 (2003).

[18] M. Catanesi et al., Eur. Phys. J. C 52, 29 (2007).

[19] D. Casper, Nucl. Phys. Proc. Suppl. 112, 161 (2002).

[20] R. Smith and E. Moniz, Nucl. Phys. B43, 605 (1972)

[21] R. Brun, F. Carminati, and S. Giani, GEANT Detector Description and Simulation Tool (1994).
[22] B. Brown et al., IEEE Nucl. Sci. Symp. Conf. Rec. 1, 652 (2004).

[23] C. Zeitnitz and T. Gabriel, Nucl. Instrum. Methods Phys. Res., Sect. A 349, 106 (1994).

[24] A. Heikkinen, N. Stepanov, and J.P. Wellisch, Bertini Intra-Nuclear Cascade Implementation in Geant4, eConf C0303241, MOMT008 (2003).

[25] J.L. A. Aunion, Ph.D. thesis, Universitat Autonoma de Barcelona 2010, fermilab-thesis-2010-45 [http://lss.fnal gov/archive/thesis/index.shtml].

[26] K. Mahn, Ph.D. thesis, Columbia University 2009, fermilab-thesis-2009-22[http://lss.fnal.gov/archive/thesis/ index.shtml.].

[27] J. Grange (MiniBooNE Collaboration), AIP Conf. Proc. 1405, 83 (2011).

[28] C. Mariani, G. Cheng, J. M. Conrad, and M. H. Shaevitz, Phys. Rev. D 84, 114021 (2011).

[29] G. Cheng et al. (SciBooNE Collaboration), Phys. Rev. D 84, 012009 (2011).

[30] N. V. Mokhov et al., arXiv:nucl-th/9812038.

[31] A. Bodek, S. Avvakumov, R. Bradford, and H. S. Budd, J. Phys. Conf. Ser. 110, 082004 (2008).

[32] J.E. Amaro, M.B. Barbaro, J.A. Caballero, and T.W. Donnelly, Phys. Rev. Lett. 108, 152501 (2012).

[33] A. Bodek, H. Budd, and M. Christy, Eur. Phys. J. C 71 , 1726 (2011).

[34] J. Nieves, I. Ruiz Simo, and M. Vicente Vacas, Phys. Lett. B 707, 72 (2012).

[35] M. Martini, M. Ericson, G. Chanfray, and J. Marteau, Phys. Rev. C 80, 065501 (2009).

[36] D. Ashery, I. Navon, G. Azuelos, and F.W. Schlepütz, Phys. Rev. C 23, 2173 (1981).

[37] M. K. Jones et al., Phys. Rev. C 48, 2800 (1993).

[38] R. D. Ransome et al., Phys. Rev. C 45, R509 (1992).

[39] K. Hiraide, Ph.D. thesis, Kyoto University 2009, fermilabthesis-2009-02 [http://lss.fnal.gov/archive/thesis/index .shtml.].

[40] G. J. Feldman and R. D. Cousins, Phys. Rev. D 57, 3873 (1998).

[41] I. Stockdale et al., Phys. Rev. Lett. 52, 1384 (1984). 